\title{
Investigation of PM Formation and Evolution in Plumes Emitted by Heavy-Duty Diesel Vehicles: Wind Tunnel Study
}

Daniele Littera

West Virginia University

Follow this and additional works at: https://researchrepository.wvu.edu/etd

\section{Recommended Citation}

Littera, Daniele, "Investigation of PM Formation and Evolution in Plumes Emitted by Heavy-Duty Diesel Vehicles: Wind Tunnel Study" (2014). Graduate Theses, Dissertations, and Problem Reports. 142. https://researchrepository.wvu.edu/etd/142

This Dissertation is protected by copyright and/or related rights. It has been brought to you by the The Research Repository @ WVU with permission from the rights-holder(s). You are free to use this Dissertation in any way that is permitted by the copyright and related rights legislation that applies to your use. For other uses you must obtain permission from the rights-holder(s) directly, unless additional rights are indicated by a Creative Commons license in the record and/ or on the work itself. This Dissertation has been accepted for inclusion in WVU Graduate Theses, Dissertations, and Problem Reports collection by an authorized administrator of The Research Repository @ WVU.

For more information, please contact researchrepository@mail.wvu.edu. 


\title{
Investigation of PM Formation and Evolution in Plumes Emitted by Heavy-Duty Diesel Vehicles: Wind Tunnel Study
}

\author{
Daniele Littera \\ Dissertation submitted \\ to the Benjamin M. Statler \\ College of Engineering and Mineral Resources \\ at West Virginia University \\ in partial fulfillment of the requirements for the degree of \\ Doctor of Philosophy in \\ Mechanical Engineering \\ Mridul Gautam, Ph.D., Chair \\ Nigel Clark, Ph.D. \\ Andrew Nix, Ph.D. \\ Gregory Thompson, Ph.D. \\ Department of Mechanical and Aerospace Engineering \\ Ettore Pennestrì, Ph.D. \\ Mechanical Engineering Department \\ College of Engineering \\ University of Rome "Tor Vergata"
}

Morgantown, West Virginia

2014

Keywords: PM, Emissions, Wind Tunnel, Heavy Duty Diesel Truck, Turbulence Copyright 2014 Daniele Littera 


\title{
ABSTRACT \\ Investigation of PM Formation and Evolution in Plumes Emitted by Heavy Duty Diesel Vehicles: Wind Tunnel Study
}

\author{
Daniele Littera, PhD in Mechanical Engineering
}

Stringent emission regulations have forced drastic technological improvements in diesel aftertreatment systems, particularly in reducing Particulate Matter (PM) emissions. Studies that have led to these technological advancements were made in controlled laboratory environments and are not representative of real-world emissions from these engines or vehicles. In addition, formation and evolution of PM from these engines are extremely sensitive to overall changes in the dilution process. In light of this, the study of the exhaust plume of a heavy-duty diesel vehicle operated inside a subsonic environmental wind tunnel can give us an idea of the dilution process and the representative emissions of the real-world scenario. The wind tunnel used for this study is capable of accommodating a full-sized heavy-duty truck and generating wind speeds in excess of 50mph. It was specifically designed and built by West Virginia University (WVU) to characterize the exhaust plume emitted from heavy-duty vehicles. A three-dimensional gantry system allows spanning the test section and sample regions in the plume with accuracy of less than $5 \mathrm{~mm}$. The investigation involves three different heavy-duty Class- 8 diesel vehicles equipped with aftertreatment technologies, representative of legacy and modern truck fleets in the USA. The three vehicles investigated are representative of three emission regulation standards, namely a US-EPA 2007 compliant, a US-EPA 2010 compliant, and a baseline vehicle without any aftertreatment technologies as a pre US-EPA 2007, respectively. The testing procedure includes three different vehicle speeds: idling, $20 \mathrm{mph}$, and $35 \mathrm{mph}$. The vehicles were tested on WVU's medium-duty chassis dynamometer, with the load applied to the truck reflecting the road load equation at the respective vehicle test speeds. Wind tunnel wind speed and vehicle speed were maintained in close proximity to one another during the entire test. Results show that the cooling and dilution of the exhaust takes place within $2 \mathrm{~m}$ from the exhaust stack. The rate of cooling and dilution are greatest in early stages of the dilution process (within $0.15 \mathrm{~m}$ from the exhaust stack) for the areas with high turbulence intensity (TI), where strong mixing phenomena occurs and the nucleation mode of the PM is formed. On the other hand, the core of the plume observes a slower cooling and dilution rate. This difference is reflected in the PM formation and evolution of these two distinct regions, as shown by the particle size distributions and number concentrations. Eventually, further downstream those heterogeneous areas due to the turbulence mix together to form a homogenous particle size distribution across the entire plume. The content of PM volatile and solid compound in the exhaust set the condition for the nucleation to occur, but the TI has the active role to generate nanoparticles. Using MARS as modeling tool of the plume enhanced the obtained notions, confirming the initial heterogeneity of the plume, further becoming homogenous. In addition, providing further detail of early stages of the plumes showing that a DPF-equipped truck generates a nucleation mode quickly adsorbed by the background PM $(0.15 \mathrm{~m}$ from the exhaust stack). 


\section{Dedication}

I would like to dedicate this work to my parents and all the people who allowed me to pursue this dream; their support has and still is fundamental to me.

Vorrei dedicare questa tesi ai miei genitori e tutte le persone che mi hanno permesso di seguire questo sogno, il loro supporto è stato ed è ancora fondamentale per me. 


\section{Acknowledgements}

The intensity and extensions of this work couldn't have been managed simply by me. I would like to thank every single graduate student, technician, engineer, and faculty that participated in this project. My special thanks goes to Dr. Mridul Gautam, who believed in this idea, and me. In addition, Dr. Daniel Carder who helped me in the everyday decisions necessary to achieve this goal. It has been a wonderful experience that taught me the value of the people surrounding me, and how their help can surprise you every day. 


\section{Table of Contents}

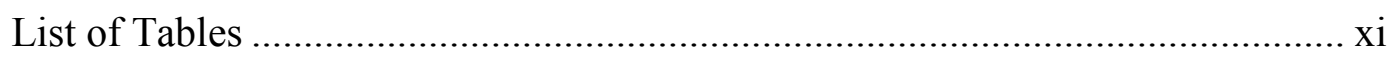

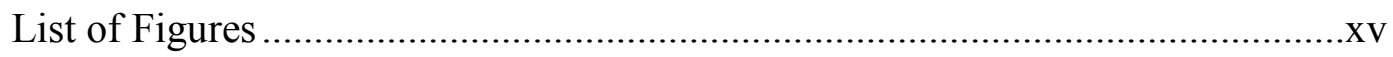

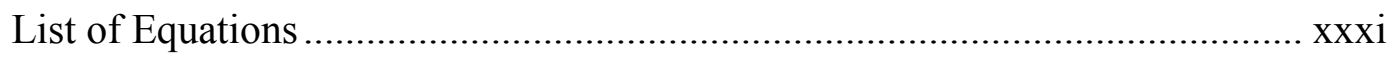

Chapter 1: Introduction ............................................................................. 1

Chapter 2: Literature Review .....................................................................6

2.1. PM and Related Health Effect ....................................................6

2.2. PM Composition and Descripting Parameters ....................................

2.3. PM Interaction with Dilution Air..................................................13

2.4. Effect of Aftertreatment Components on PM emissions ......................17

2.5. PM Measurements in Laboratory and On-Road Conditions.................18

2.6. PM Measurements in Road-Side Studies............................................20

2.7. Comparison of PM measurement in Laboratory and Real World .........21

2.8. PM Measurements in Wind Tunnel .............................................25 
Chapter 3: Design and Construction of the WVU Non-Recirculating Environmental

Subsonic Full Scale Wind Tunnel........................................................2

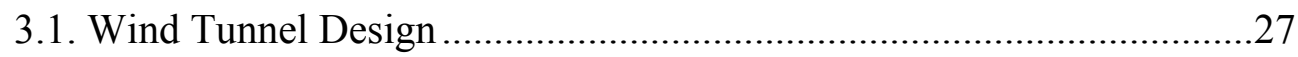

3.1.1. Design Constraint and Target ...........................................27

3.1.2. Computational Fluid-dynamic Analyses................................30

3.1.3. Finite Element Method Structural Analyses ..........................41

3.1.3.1. Geometry Definition .............................................42

3.1.3.2. Material Properties .....................................................46

3.1.3.3. Element Properties ....................................................4 48

3.1.3.4. Mesh Definition .........................................................49

3.1.3.5. Load Sets and Constraint Sets.....................................50

3.1.3.6. Structural Results .......................................................52

3.2. Wind Tunnel Construction...............................................................61

3.3 Wind Tunnel Qualification and Commissioning ...............................68

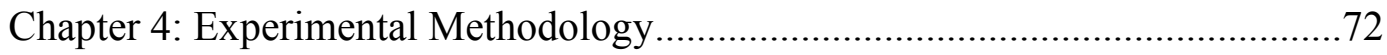

4.1. Instruments, Sampling Procedures, and QA/QC ...............................72

4.1.1. Plume Instruments ..................................................... 76

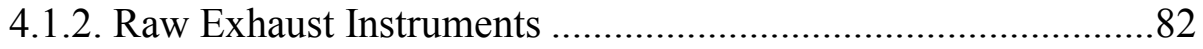


4.1.3. Background Air Instruments .88

4.1.4. Transportable Heavy-Duty Vehicle Emissions Testing Laboratory

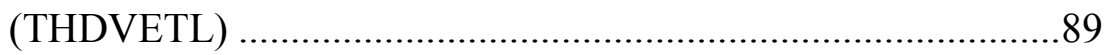

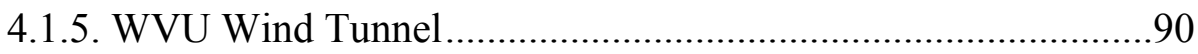

4.1.6. Sampling Plume Pattern..........................................................93

4.1.7. Wind Tunnel Coordinate Reference System...........................96

4.1.8. Data File Dictionary.........................................................96

4.1.9. WVU QA/QC Assurance Plan.............................................100

4.2. Class-8 Heavy-duty Diesel Vehicles ................................................105

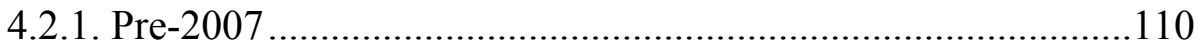

4.2.2. Post-2007 …................................................................. 111

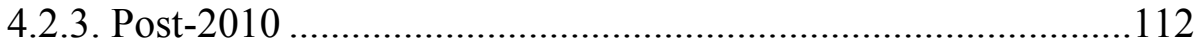

4.3. Test Matrix ................................................................................. 113

Chapter 5: Characterization of Differences in Concentrations and Size Distributions of PM Emissions from Three US-EPA Regulation Compliant HDDTs at Different

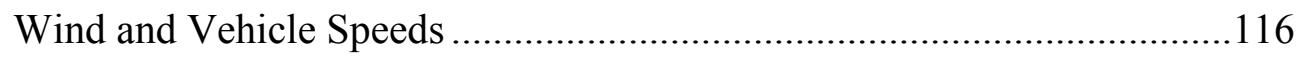

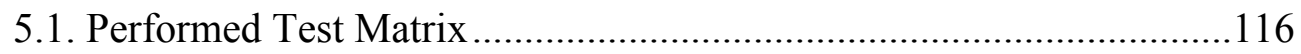

5.2. Background PM Air and Raw Emissions ......................................116 


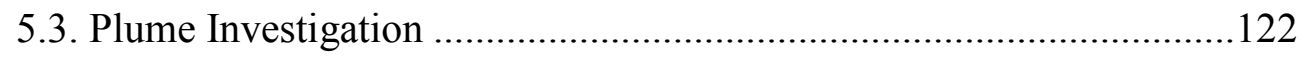

5.3.1 Pre-2007 Vehicle Test Results.................................................124

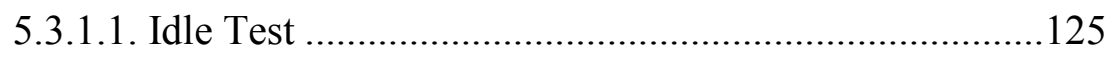

5.3.1.2. 20mph Test...............................................................134

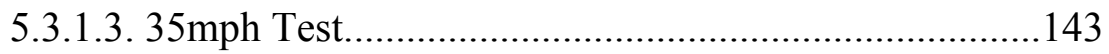

5.3.2. Post-2007 Vehicle Test Results .................................................148

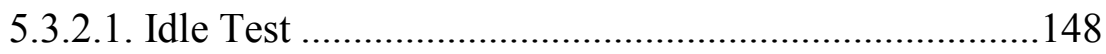

5.3.2.2. 20mph Test................................................................153

5.3.2.3. 35mph Test.................................................................. 161

5.3.3. Post-2010 Vehicle Test Vehicle..................................................167

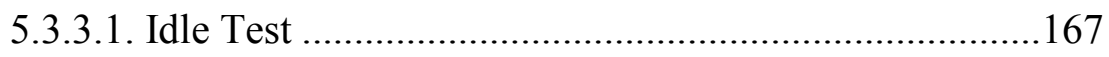

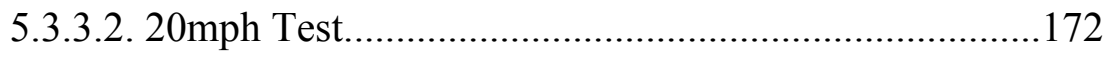

5.3.3.3. 35mph Test..............................................................174

5.4. Comparison of PM Plume Measurements with Standardized Laboratory

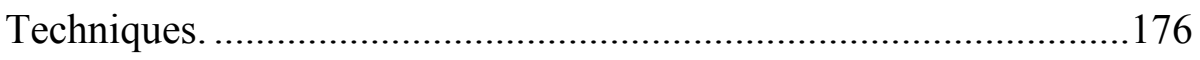

5.4.1. Pre-2007 Vehicle Comparison Results ......................................176

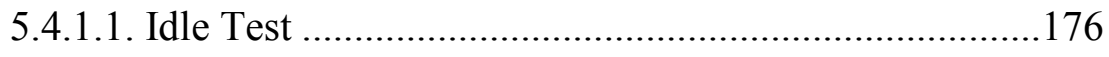

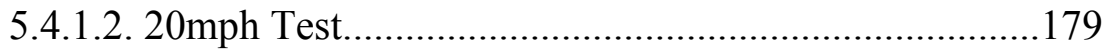

viii 
5.4.1.3. 35mph Test...............................................................181

5.4.2. Post-2007 Vehicle Comparison Results....................................183

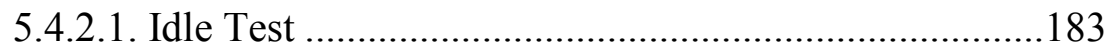

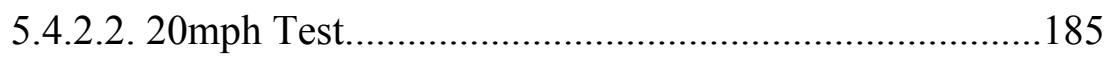

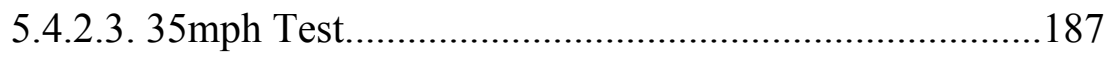

5.4.3. Post-2010 Vehicle Comparison Results.....................................189

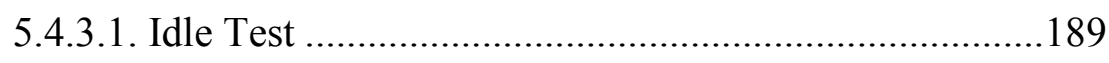

5.4.3.2. 20mph Test................................................................191

5.4.3.3. 35mph Test.................................................................193

Chapter 6: Detailed Plume Modeling via 3D MARS .........................................195

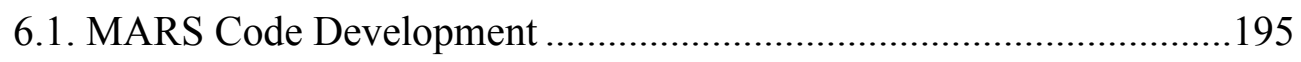

6.2. Comparison of PM Particle Size Distribution and Concentration Upon Paths with Predominant Nucleation and Accumulation Mode.....................198

6.2.1. Pre-2007 Vehicle MARS Results .............................................198

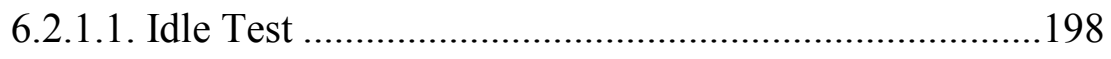

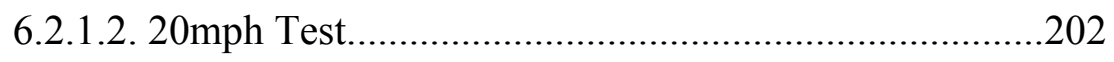

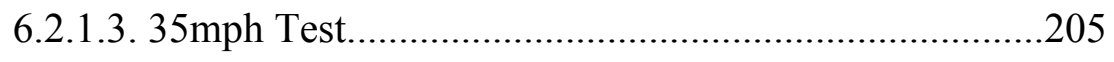

6.2.2. Post-2007 Vehicle MARS Results..........................................209

ix 
6.2.2.1. Idle Test

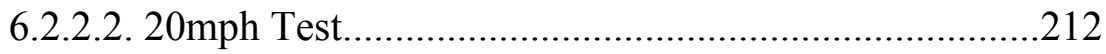

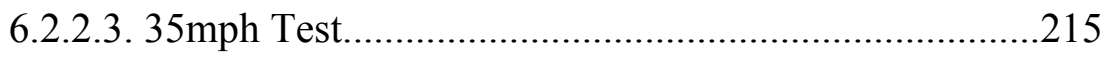

6.2.3. Post-2010 Vehicle MARS Results..............................................218

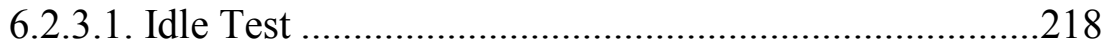

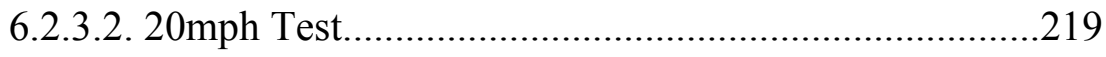

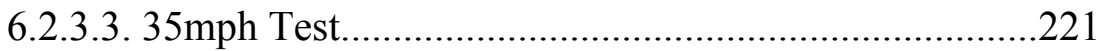

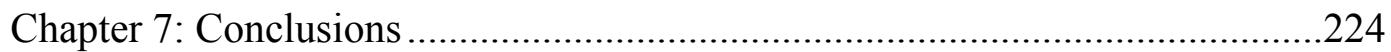

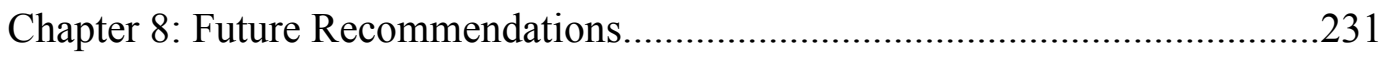

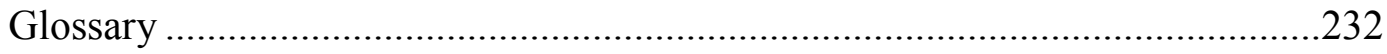

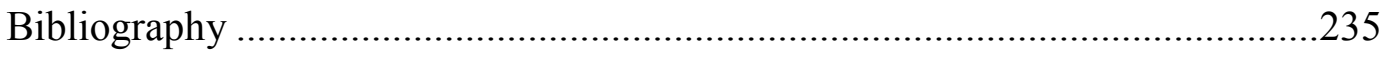

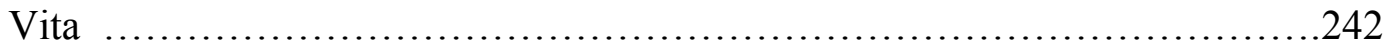




\section{List of Tables}

Table 3-1: Wind tunnel natural frequencies analysis and comparison of the excitation frequency during operational conditions ..........................................57

Table 3-2: Wind Tunnel Wood Main Structural Components..................................62

Table 3-3: Wind Tunnel Metal Main Structural Components ..................................62

Table 3-4: Engine Propeller Specifications .......................................................66

Table 3-5: Wind Tunnel Propeller Specifications ..................................................67

Table 3-6: Wind Speed Variation Between the Core and Outer Region for the Qualification Test at 35mph Sind Speed. .........................................69

Table 4-1: Span Calibration Gases for the Sampling Cart Gaseous Instruments for Pre2007 and Post-2007 Truck ……………………………………........

Table 4-2: Span Calibration Gases for the Sampling Cart Gaseous Instruments for Post2010 Truck .79

Table 4-3: Span Calibration Gases for the TREL Gaseous Instruments for Pre-2007 Truck .84

Table 4-4: Span Calibration Gases for the TREL Gaseous Instruments for Post-2007 Truck 85

Table 4-5: Span Calibration Gases for the TREL Gaseous Instruments for Post-2010 Truck .85

Table 4-6: PM Spectrometer Used for the Different Tests ......................................87

Table 4-7: WVU Wind Tunnel Specifications.....................................................90

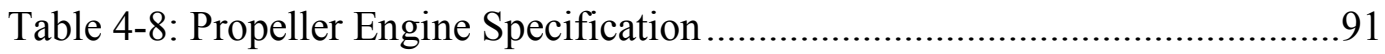

Table 4-9: Wind Tunnel Propeller Specifications ..............................................92 
Table 4-10: Sampling Plume Pattern Parameters .95

Table 4-11: Data File Dictionary .97

Table 4-12: US-EPA Emissions Standards for On-Road Heavy Duty Diesel Engines (HDDE) regarding the tested vehicles, the emissions are indicated in gr/bhp-hr. 106

Table 4-13: Pre-2007 vehicle specifications 110

Table 4-14: Pre-2007 engine specification (*certification values) 110

Table 4-15: Post-2007 vehicle specifications 111

Table 4-16: Post-2007 engine specification (*certification values) 111

Table 4-17: Post-2010 vehicle specifications 112

Table 4-18: Post-2010 engine specifications (*certification values) ..................112

Table 4-19: Test Campaign Matrix 114

Table 5-1: Background Air $\mathrm{CO}_{2}$ and $\mathrm{NO}_{\mathrm{x}}$ Emissions Concentration Averaged Over the Nine Tests. 119

Table 5-2: Raw Tailpipe Gaseous and PM Soot Emissions for the Three Tested Vehicles Measured during the Idle Test 120

Table 5-3: Raw Tailpipe Gaseous and PM Soot Emissions for the Three Tested Vehicles Measured During the 20mph Test.

Table 5-4: Raw Tailpipe Gaseous and PM Soot Emissions for the Three Tested Vehicles Measured During the 35mph Test 121

Table 5-5: Vehicle and Dynamometer Parameter during the idle test for the Pre-2007 Vehicle. 125

Table 5-6: Vehicle and Dynamometer Parameter During the 20mph Test for the Pre2007 Vehicle. 134 
Table 5-7: Vehicle and Dynamometer Parameter During the $35 \mathrm{mph}$ Test for the Pre2007 Vehicle. 143

Table 5-8: Vehicle and Dynamometer Parameter During the Idle Test for the Post-2007 Vehicle. 148

Table 5-9: Vehicle and Dynamometer Parameters During the 20mph Test for the Post2007 Vehicle. 153

Table 5-10 Vehicle and Dynamometer Parameter During the 35mph Test for the Post2007 Vehicle. 162

Table 5-11: Vehicle and Dynamometer Parameter During the Idle Test for the Post2010 Vehicle. 168

Table 5-12: Vehicle and Dynamometer Parameters During the 20mph Test for the Post2010 Vehicle. 172

Table 5-13: Vehicle and Dynamometer Parameters During the 35mph Test for the Post2010 Vehicle. 174

Table 5-14: DDS Parameters Used During the PM Particle Size Distribution and Concentration Sampling from Raw Exhaust for the Pre-2007 Vehicle in the Idle Test. 177

Table 5-15: DDS Parameters Used During the PM Particle Size Distribution and Concentration Sampling from Raw Exhaust for the Pre-2007 Vehicle in the $20 \mathrm{mph}$ Test 179

Table 5-16: DDS Parameters Used During the PM Particle Size Distribution and Concentration Sampling from Raw Exhaust for the Pre-2007 Vehicle in the $35 \mathrm{mph}$ Test. 181 
Table 5-17: DDS Parameters Used During the PM Particle Size Distribution and Concentration Sampling from Raw Exhaust for the Post-2007 Vehicle in the Idle Test. 183

Table 5-18: DDS Parameters Used During the PM Particle Size Distribution and Concentration Sampling from Raw Exhaust for the Post-2007 Vehicle in the $20 \mathrm{mph}$ Test 185

Table 5-19: DDS Parameters Used During the PM Particle Size Distribution and Concentration for the Post-2007 Vehicle in the 35mph Test. 187

Table 5-20: DDS Parameters Used During the PM Particle Size Distribution and Concentration for the Post-2010 Vehicle in the 35mph Test. 189

Table 5-21: DDS Parameters Used During the PM Particle Size Distribution and Concentration Measurement for the 20mph Test and Post-2010 Vehicle. 191

Table 5-22: DDS Parameters Used During the PM Particle Size Distribution and Concentration Measurement for the 35mph Test and Post-2010 Vehicle. 193 


\section{List of Figures}

Figure 2-1: Typical composition and structure of engine exhaust particles (Kittlelson,

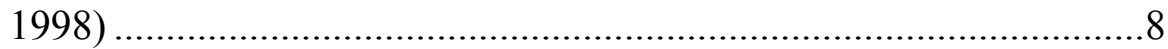

Figure 2-2: Typical particle composition for a HDDE tested in a heavy-duty transient cycle (Kittlelson, 1998) .9

Figure 2-3: Typical engine exhaust size distribution both mass and number weights are shown (Kittlelson, 1998). 10

Figure 2-4: Influence of Solid Particles on Saturation Ratio during Adiabatic Dilution (Kittelson, et al., 1999) 12

Figure 2-5: Box-model calculation of the evolution of the sum of 10 aerosol distributions by coagulation when (a) particles are assumed spherical and no van der Waals or viscous forces are acting, (b) particles are assumed spherical and van der Waals and viscous forces are acting, and (c) particles are assumed fractal and Van der Waals and viscous forces are acting (Jacobson, et al., 2004). 15

Figure 3-1: CAD cut view representation of the WVU wind tunnel .28

Figure 3-2: CFD simulations, exhaust stack lower than fairing with 35mph wind speed 31

Figure 3-3: CFD simulations, exhaust stack higher than fairing with 35mph wind speed 32

Figure 3-4: WVU wind tunnel entrance section and relative test cell cross section dimensions 
Figure 3-5: Pathlines colored by velocity magnitude, released from $10 \mathrm{~m}$ surface (4th

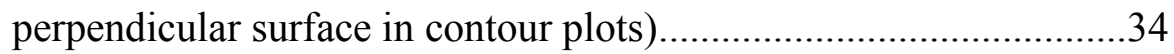

Figure 3-6 Side view of the velocity contours on the tunnel centerline $(\mathrm{m} / \mathrm{s})$. The simulated wind speed is $35 \mathrm{mph}$. 35

Figure 3-7: Velocity contours on the tunnel centerline, $(\mathrm{m} / \mathrm{s})$ crossections are at the point of tunnel contraction and $5 \mathrm{~m}, 10 \mathrm{~m}, 15 \mathrm{~m}$, and $20 \mathrm{~m}$ downstream with one additional at the back of the cab. .36

Figure 3-8: Boundary layer and displacement thickness growth within the WVU wind tunnel for a simulated wind speed of $35 \mathrm{mph}$. 39

Figure 3-9: Boundary layer and displacement thickness for different simulated wind speeds at $30 \mathrm{~m}$ downstream the tunnel entrance.

Figure 3-10: Wind tunnel CAD model for structural analyses during SolidWorks ${ }^{\circledR}$ generation, $3 \mathrm{D}$ view

Figure 3-11. Wind tunnel CAD model for structural analyses during Solidworks ${ }^{\circledR}$ generation, front view 43

Figure 3-12: Wind tunnel CAD model for structural analyses after being imported in Femap(C, 3D view shows the propeller section 44

Figure 3-13: Wind tunnel CAD model for structural analyses after being imported in Femap $($, 3D view shows the cart corridor and the slot in the ceiling45

Figure 3-14: Wind tunnel CAD model for structural analyses after being imported in Femap $\subset$, 3D view shows the access door and the screen 45

Figure 3-15: Wind tunnel CAD model for structural analyses after being imported in Femap $($, 3D view shows the entire geometry. 46 
Figure 3-16: Femap® screenshot indicating the material properties for the structural steel

Figure 3-17: Femap $($ screenshot indicating the material properties for the structural pine wood 48

Figure 3-18: Femap $\bigodot$ screenshot of the general structural laminate element properties .49

Figure 3-19: Femap@ screenshot illustrating the mesh for the structural analyses, it is a visible well-structured mesh 50

Figure 3-20: Operational conditions structural analysis results, overview of the entire model. 53

Figure 3-21: Operational conditions structural analysis results, detail of the propeller convergent section 53

Figure 3-22: Operational conditions structural analysis results, detail of door and screen 54

Figure 3-23: Operational conditions structural analysis results, detail of the entrance section and instrument cart corridor .54

Figure 3-24: Snow accumulation conditions structural analysis results, overview of the entire model 55

Figure 3-25: Snow accumulation conditions structural analysis results, detail of the entrance section, instrument cart corridor and entrance door with screen 56

Figure 3-26: Lateral wind conditions structural analysis results, overview of the entire model. 56 
Figure 3-27: Lateral wind conditions structural analysis results, detail of the entrance section, instrument cart corridor and entrance door with screen .......57

Figure 3-28: First natural frequency for the wind tunnel, the deformation is showed with an amplification factor of 30 . .59

Figure 3-29: Wind tunnel buckling results, details of the propeller convergent ...60

Figure 3-30: View of the internal wall of the wind tunnel, details of the door opening and king stud reinforcement 63

Figure 3-31: Fabrication of the wind tunnel wood trusses, details of the 1/2" plywood gusset reinforcement of the joints 64

Figure 3-32: Wind tunnel wall installation at the construction site 64

Figure 3-33: Overview of the finished wood main structure, metal siding, and roofing .65

Figure 3-34: Overview of the installation of the metal main structure 65

Figure 3-35: Installation of the propeller engine on its final setup 67

Figure 3-36: Wind speed measurements during the qualification test at $35 \mathrm{mph}$ at the $15 \mathrm{~m}$ plane. The white circles represent the sampling points. .70

Figure 3-37: TI measurements during the qualification test at $35 \mathrm{mph}$ at the $15 \mathrm{~m}$ plane. The white circles represent the sampling points. 71

Figure 4-1: Wind tunnel, chassis dynamometer, and TREL setup 73

Figure 4-2: Internal view of the wind tunnel with mock-up truck and sampling gantry .74

Figure 4-3: Experimental setup schematic. .75

Figure 4-4: Exhaust plume sampling probe and flow field characterization sensors77 
Figure 4-5: PM penetration efficiency for the heated sampling line at a flow rate of 5slpm for a particle size spectrum wider than the typical Diesel PM81 Figure 4-6: WVU Transportable Raw Emissions Laboratory (TREL)...................84

Figure 4-7: Sampling plume pattern for each plane and the path followed by the sampling probe. 94

Figure 4-8: Initial identification of the plume sampling points 95

Figure 4-9: Wind tunnel coordinate reference system .96

Figure 5-1: PM particle size distribution and concentration for the background air entering the wind tunnel during three different vehicle and wind speed tests. Results indicate almost constant PM background air. 118

Figure 5-2: Longitudinal vertical contour cut plot of the $\mathrm{NO}_{\mathrm{x}}$ trace for the Pre-2007 vehicle during the idle test. The plane is passing through the exhaust stack.

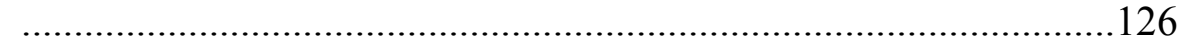

Figure 5-3: Longitudinal vertical contour cut plot of the turbulence intensity trace for the Pre-2007 vehicle during the idle test. The plane is passing through the exhaust stack. 127

Figure 5-4: Transverse contour cut plot of $\mathrm{CO} 2$, NOx, wind speed, and TI. The plane of this plot is sampling plane 2 , which is $0.315 \mathrm{~m}$ from the emitting point for the idle test of the Pre-2007 vehicle. The white dots represent the defining plane sampling points. 128 
Figure 5-5: Transverse contour cut plot of DustTrack, total PM Count tailpipe corrected, nucleation mode PM count, and accumulation mode PM count for the idle test of the Pre-2007 vehicle. The plane of this plot is sampling plane 2 , which is $0.315 \mathrm{~m}$ from the emitting point. The white dots represent the plane sampling points

Figure 5-6: Particle size distribution of point 12 and point 13 of plane $2(0.315 \mathrm{~m}$ downstream) for the idle test of the Pre-2007 vehicle.

Figure 5-7: Transverse contour cut plot of DustTrack, total PM count tailpipe corrected, nucleation mode PM count, and accumulation mode PM count for the idle test of the Pre-2007 vehicle. The plane of this plot is sampling plane 9, which is $6.815 \mathrm{~m}$ from the emitting point. The white dots represent the plane sampling points. 132

Figure 5-8: Particle size distribution of point 9, point 10, and point 12 of plane 9 (6.815m downstream) for the idle test of the Pre-2007 vehicle......133

Figure 5-9: Longitudinal vertical contour cut plot of the NOx trace for the Pre-2007 vehicle during the $20 \mathrm{mph}$ test. The plane is passing through the exhaust stack. 135

Figure 5-10: Longitudinal vertical contour cut plot of the turbulence intensity trace for the Pre-2007 vehicle during the $20 \mathrm{mph}$ test. The plane is passing through the exhaust stack. 136

Figure 5-11: Transverse contour cut plot of CO2, NOx, wind speed, and TI. The plane of this plot is sampling plane 1 , which is $0.115 \mathrm{~m}$ from the emitting point for the $20 \mathrm{mph}$ test of the Pre-2007 vehicle. The white dots represent the defining plane sampling points. 
Figure 5-12: Transverse contour cut plot of DustTrack, total PM Count tailpipe corrected, nucleation mode PM count, and accumulation mode PM count for the 20mph test of the Pre-2007 vehicle. The plane of this plot is sampling plane 1 , which is $0.115 \mathrm{~m}$ from the emitting point. The white dots represent the plane sampling points. 138

Figure 5-13: Particle size distribution of point 1 and point 10 of plane $1(0.115 \mathrm{~m}$ downstream) for the $20 \mathrm{mph}$ test of the Pre-2007 vehicle. 139

Figure 5-14: Transverse contour cut plot of CO2, NOx, wind speed, and TI. The plane of this plot is sampling plane 7 , which is $2.815 \mathrm{~m}$ from the emitting point for the $20 \mathrm{mph}$ test of the Pre-2007 vehicle. The white dots represent the defining plane sampling points 140

Figure 5-15: Transverse contour cut plot of DustTrack, total PM count tailpipe corrected, nucleation mode PM count, and accumulation mode PM count for the $20 \mathrm{mph}$ test of the Pre-2007 vehicle. The plane of this plot is sampling plane 7 , which is $2.815 \mathrm{~m}$ from the emitting point. The white dots represent the plane sampling points. 141

Figure 5-16: Particle size distribution of point 1, point 9, and point 10 of plane 7 (2.815m downstream) for the $20 \mathrm{mph}$ test of the Pre-2007 vehicle. 142

Figure 5-17: Longitudinal vertical contour cut plot of the NOx trace for the Pre-2007 vehicle during the $35 \mathrm{mph}$ test. The plane is passing through the exhaust stack. 144

Figure 5-18: Longitudinal vertical contour cut plot of the turbulence intensity trace for the Pre-2007 vehicle during the $35 \mathrm{mph}$ test. The plane is passing through the exhaust stack. 
Figure 5-19: Transverse contour cut plot of CO2, NOx, wind speed, and TI. The plane of this plot is sampling plane 5 , which is $1.315 \mathrm{~m}$ from the emitting point for the 35mph test of the Pre-2007 vehicle. The white dots represent the defining plane sampling point. 146

Figure 5-20: Particle size distribution of point 1 , point 9 , and point 10 of plane 5 (1.315m downstream) for the 20mph test of the Pre-2007 vehicle.147

Figure 5-21: Transverse contour cut plot of DustTrack, total PM count tailpipe corrected, nucleation mode PM count, and accumulation mode PM count for the idle test of the Post-2007 vehicle. The plane of this plot is sampling plane 2 , which is $0.315 \mathrm{~m}$ from the emitting point. The white dots represent the plane sampling points 150

Figure 5-22: Particle size distribution of point 1 , and point 8 of plane $2(0.315 \mathrm{~m}$ downstream) for the idle test of the Post-2007 vehicle.

Figure 5-23 Particle size distribution of point 1 , and point 10 of plane $4(0.815 \mathrm{~m}$ downstream) for the idle test of the Post-2007 vehicle. 152

Figure 5-24: Transverse contour cut plot of DustTrack, total PM count tailpipe corrected, nucleation mode PM count, and accumulation mode PM count for the 20mph test of the Post-2007 vehicle. The plane of this plot is sampling plane 2 , which is $0.315 \mathrm{~m}$ from the emitting point. The white dots represent the plane sampling points.

Figure 5-25: Particle size distribution of point 12 , and point 13 of plane $2(0.315 \mathrm{~m}$ downstream) for the 20mph test of the Post-2007 vehicle. 155 
Figure 5-26: Transverse contour cut plot of $\mathrm{CO} 2, \mathrm{NOx}$, wind speed, and TI. The plane of this plot is sampling plane 6 , which is $1.815 \mathrm{~m}$ from the emitting point for the $20 \mathrm{mph}$ test of the Pre-2007 vehicle. The white dots represent the defining plane sampling points. 157

Figure 5-27: Transverse contour cut plot of DustTrack, total PM count tailpipe corrected, nucleation mode PM count, and accumulation mode PM count for the 20mph test of the Post-2007 vehicle. The plane of this plot is sampling plane 6 , which is $1.815 \mathrm{~m}$ from the emitting point. The white dots represent the plane sampling points.

Figure 5-28: Particle size distribution of point 8 , and point 12 of plane $6(1.815 \mathrm{~m}$ downstream) for the $20 \mathrm{mph}$ test of the Post-2007 vehicle. 160

Figure 5-29: Particle size distribution of point 12 of plane 6 (1.815m downstream) and point 12 of plane 5 (1.315m downstream) for the $20 \mathrm{mph}$ test of the Post2007 vehicle. 161

Figure 5-30: Transverse contour cut plot of DustTrack, total PM count tailpipe corrected, nucleation mode PM count, and accumulation mode PM count for the $35 \mathrm{mph}$ test of the Post-2007 vehicle. The plane of this plot is sampling plane 2, which is $0.315 \mathrm{~m}$ from the emitting point. The white dots represent the plane sampling points. 163

Figure 5-31: Particle size distribution of point 1, point 2, and point 10 of plane 2 ( $0.315 \mathrm{~m}$ downstream) for the $20 \mathrm{mph}$ test of the Pre-2007 vehicle. 164 
Figure 5-32: Transverse contour cut plot of DustTrack, total PM count tailpipe corrected, nucleation mode PM count, and accumulation mode PM count for the $35 \mathrm{mph}$ test of the Post-2007 vehicle. The plane of this plot is sampling plane 7 , which is $2.815 \mathrm{~m}$ from the emitting point. The white dots represent the plane sampling points. 165

Figure 5-33: Particle size distribution of point 8 , point 9 , and point 10 of plane 7 ( $2.815 \mathrm{~m}$ downstream) for the $35 \mathrm{mph}$ test of the Post-2007 vehicle. 166

Figure 5-34: Transverse contour cut plot of $\mathrm{CO} 2$, NOx, wind speed, and TI. The plane of this plot is sampling plane 2 , which is $0.315 \mathrm{~m}$ from the emitting point for the idle test of the Post-2010 vehicle. The white dots represent the defining plane sampling points. 169

Figure 5-35: Transverse contour cut plot of DustTrack, total PM count tailpipe corrected, nucleation mode PM count, and accumulation mode PM count for the idle test of the Post-2010 vehicle. The plane of this plot is sampling plane 2 , which is $0.315 \mathrm{~m}$ from the emitting point. The white dots represent the plane sampling points 170

Figure 5-36: Particle size distribution of point 1 , and point 8 of plane $2(0.315 \mathrm{~m}$ downstream) for the idle test of the Post-2010 vehicle 171

Figure 5-37: Transverse contour cut plot of DustTrack, total PM count tailpipe corrected, nucleation mode PM count, and accumulation mode PM count for the $20 \mathrm{mph}$ test of the Post-2010 vehicle. The plane of this plot is sampling plane 2, which is $0.315 \mathrm{~m}$ from the emitting point. The white dots represent the plane sampling points. 173 
Figure 5-38: Transverse contour cut plot of DustTrack, total PM count tailpipe corrected, nucleation mode PM count, and accumulation mode PM count for the $35 \mathrm{mph}$ test of the Post-2010 vehicle. The plane of this plot is sampling plane 2, which is $0.315 \mathrm{~m}$ from the emitting point. The white dots represent the plane sampling points. 175

Figure 5-39: PM particle size distribution and concentration comparison between the DMS DDS raw exhaust sampling and plume measurements for the Pre2007 vehicle in the idle test. Plane 4 point 12, plane 6 point 12, and plane 6 point 13 present the closer DR to the raw exhaust. 178

Figure 5-40: PM particle size distribution and concentration comparison between the DMS DDS raw exhaust sampling and plume measurements for the Pre2007 vehicle in the $20 \mathrm{mph}$ Test. Plane 4 point 9, plane 6 point 11, and plane 6 point 12 present the closer DR to the raw exhaust. 180

Figure 5-41: PM particle size distribution and concentration comparison between the SMPS DDS raw exhaust sampling and plume measurements for the Pre2007 vehicle in the $35 \mathrm{mph}$ test. Plane 5 point 11 , plane 6 point 10 , and plane 10 point 11 present the closer DR to the raw exhaust. 182

Figure 5-42: PM particle size distribution and concentration comparison between the DMS and SMPS DDS raw exhaust sampling, and plume measurements for the Post-2007 Vehicle in the idle test. Plane 1 point 2, plane 3 point 2, and plane 4 point 2 present the closer DR to the raw exhaust. .......184 
Figure 5-43: PM particle size distribution and concentration comparison between the DMS and SMPS DDS raw exhaust sampling, and plume measurements for the Post-2007 vehicle in the 20mph test. Plane 5 point 10, plane 5 point 13, and plane 6 point 10 present the closer DR to the raw exhaust.186

Figure 5-44: PM particle size distribution and concentration comparison between the DMS and SMPS DDS raw exhaust sampling, and plume measurements for the Post-2007 vehicle in the $35 \mathrm{mph}$ test. Plane 4 point 11, plane 5 point 9, and plane 5 point 10 present the closer DR to the raw exhaust. .188

Figure 5-45: PM particle size distribution and concentration comparison between the SMPS DDS raw exhaust sampling and plume measurements for the Post2010 vehicle in the idle test. Plane 2 point 2, plane 4 point 10, and plane 5 point 10 present the closer DR to the raw exhaust.

Figure 5-46: PM particle size distribution and concentration comparison between the SMPS DDS raw exhaust sampling and plume measurements for the Post2010 vehicle in the $20 \mathrm{mph}$ test. Plane 4 point 3 , plane 4 point 12 , and plane 6 point 13 present the closer DR to the raw exhaust. 192

Figure 5-47: PM particle size distribution and concentration comparison between the SMPS DDS raw exhaust sampling and plume measurements for the Post2010 vehicle in the $35 \mathrm{mph}$ test. Plane 3 point 9, plane 3 point 12 present the closer DR to the raw exhaust.

Figure 6-1: Residual sum of squares analysis for the 48 channels for the Pre-2007 vehicle during the idle test. 
Figure 6-2: Particle size distribution and concentration for plane 1 and plane 7 of the TI path and core plume path (respectively $0.115 \mathrm{~m}$ and $2.815 \mathrm{~m}$ from the exhaust stack). This plot represent the results of the MARS model for the Pre-2007 vehicle during the idle test. 200

Figure 6-3: Particle size distribution and concentration for plane 1 to plane 7 of the TI path and core plume path (respectively from $0.115 \mathrm{~m}$ to $2.815 \mathrm{~m}$ after the exhaust stack). This plot represent the results of the MARS model for the Pre-2007 vehicle during the idle test. 201

Figure 6-4: Residual sum of squares analysis for the 48 channels for the Pre-2007 vehicle during the $20 \mathrm{mph}$ test. 202

Figure 6-5: Particle size distribution and concentration for plane 1 and plane 7 of the TI path and core plume path (respectively $0.115 \mathrm{~m}$ and $2.815 \mathrm{~m}$ from the exhaust stack). This plot represents the results of the MARS model for the Pre-2007 vehicle during the $20 \mathrm{mph}$ test. .203

Figure 6-6: Particle size distribution and concentration for plane 1 to plane 7 of the TI path and core plume path (respectively from $0.115 \mathrm{~m}$ to $2.815 \mathrm{~m}$ after the exhaust stack). This plot represents the results of the MARS model for the Pre-2007 vehicle during the $20 \mathrm{mph}$ test. 204

Figure 6-7: Residual sum of squares analysis for the 48 channels for the Pre-2007 vehicle during the $35 \mathrm{mph}$ test. .206

Figure 6-8: Particle size distribution and concentration for plane 1 and plane 7 of the TI path and core plume path (respectively $0.115 \mathrm{~m}$ and $2.815 \mathrm{~m}$ from the exhaust stack). This plot represents the results of the MARS model for the Pre-2007 vehicle during the $35 \mathrm{mph}$ test. .207 
Figure 6-9: Particle size distribution and concentration for plane 1 to plane 7 of the TI path and core plume path (respectively from $0.115 \mathrm{~m}$ to $2.815 \mathrm{~m}$ after the exhaust stack). This plot represents the results of the MARS model for the Pre-2007 vehicle during the $35 \mathrm{mph}$ test. 208

Figure 6-10: Residual sum of squares analysis for the 48 channels for the Post-2007 vehicle during the idle test. 209

Figure 6-11: Particle size distribution and concentration for plane 1 and plane 7 of the TI path and core plume path (respectively $0.115 \mathrm{~m}$ and $2.815 \mathrm{~m}$ from the exhaust stack). This plot represents the results of the MARS model for the Post-2007 vehicle during the idle test 210

Figure 6-12: Particle size distribution and concentration for plane 1 to plane 7 of the TI path and core plume path (respectively from $0.115 \mathrm{~m}$ to $2.815 \mathrm{~m}$ after the exhaust stack). This plot represents the results of the MARS model for the Post-2007 vehicle during the idle test. 211

Figure 6-13: Residual sum of squares analysis for the 48 channels for the Post-2007 vehicle during the $20 \mathrm{mph}$ test. 212

Figure 6-14: Particle size distribution and concentration for plane 1 and plane 7 of the TI path and core plume path (respectively $0.115 \mathrm{~m}$ and $2.815 \mathrm{~m}$ from the exhaust stack). This plot represents the results of the MARS model for the Post-2007 vehicle during the $20 \mathrm{mph}$ test .213

Figure 6-15: Particle size distribution and concentration for plane 1 to plane 7 of the TI path and core plume path (respectively from $0.115 \mathrm{~m}$ to $2.815 \mathrm{~m}$ after the exhaust stack). This plot represents the results of the MARS model for the Post-2007 vehicle during the $20 \mathrm{mph}$ test. 214

xxviii 
Figure 6-16: Residual sum of squares analysis for the 48 channels for the Post-2007 vehicle during the $35 \mathrm{mph}$ test. 215

Figure 6-17: Particle size distribution and concentration for plane 1 and plane 7 of the TI path and core plume path (respectively $0.115 \mathrm{~m}$ and $2.815 \mathrm{~m}$ from the exhaust stack). This plot represents the results of the MARS model for the Post-2007 vehicle during the $35 \mathrm{mph}$ test. .216

Figure 6-18: Particle size distribution and concentration for plane 1 to plane 7 of the TI path and core plume path (respectively from $0.115 \mathrm{~m}$ to $2.815 \mathrm{~m}$ after the exhaust stack). This plot represents the results of the MARS model for the Post-2007 vehicle during the $35 \mathrm{mph}$ test 217

Figure 6-19: Residual sum of squares analysis for the 48 channels for the Post-2010 vehicle during the idle test. 218

Figure 6-20: Residual sum of squares analysis for the 48 channels for the Post-2010 vehicle during the $20 \mathrm{mph}$ test. 219

Figure 6-21: Particle size distribution and concentration for plane 1 and plane 7 of the TI path and core plume path (respectively $0.115 \mathrm{~m}$ and $2.815 \mathrm{~m}$ from the exhaust stack). This plot represent the results of the MARS model for the Post-2010 vehicle during the $20 \mathrm{mph}$ test 220

Figure 6-22 Residual sum of squares analysis for the 48 channels for the Post-2010 vehicle during the idle test. 221

Figure 6-23 Particle size distribution and concentration for plane 1 and plane 7 of the TI path and core plume path (respectively $0.115 \mathrm{~m}$ and $2.815 \mathrm{~m}$ from the exhaust stack). This plot represents the results of the MARS model for the Post-2010 vehicle during the $35 \mathrm{mph}$ test. 222

$$
\text { xxix }
$$


Figure 6-24: Particle size distribution and concentration for plane 1 to plane 7 of the TI path and core plume path (respectively from $0.115 \mathrm{~m}$ to $2.815 \mathrm{~m}$ after the exhaust stack). This plot represents the results of the MARS model for the Post-2010 vehicle during the $35 \mathrm{mph}$ test.....................................223 


\section{List of Equations}

Equation 3-1 37

Equation 3-2 37

Equation 3-3 37

Equation 4-1 113

xxxi 


\section{Chapter 1: Introduction}

Over the past decade, heavy-duty diesel engine (HDDE) gaseous and PM emissions have significantly reduced (Englert, 2004) (Ardanese, et al., 2009) (Littera, et al., 2011). These reductions were achieved on the basis of measurements conducted mainly in laboratories equipped with constant volume sampling (CVS) systems, following standard conditions defined by the code of federal regulations (CFR). Limited extent on-road chase studies augmented the laboratory tests. Engine manufactures have adopted multiple pathways to meet the continuously evolving heavy-duty emissions standards.

Recent technology advancements in HDDEs include advanced combustion strategies for increased engine efficiency, diesel oxidation catalysts (DOC) to reduce the emissions of hydrocarbons (HC) and carbon monoxide (CO), diesel particulate filters (DPFs) for particulate matter (PM) control, and selective catalyst reduction (SCR) for control of oxides of nitrogen (NOx) emissions. The deployment of DOC, DPF, and SCR has proven highly effective in reducing emissions. The wall flow filter is capable of trapping $99 \%$ of the PM mass emitted by the engine (Littera, et al., 2012). SCR technology with aqueous urea injection has been identified as the preferred technology for $\mathrm{NO}_{\mathrm{x}}$ mitigation from HDDEs. However, SCR technology is plagued by temperature requirements of $250^{\circ} \mathrm{C}$ and above for optimal operating conditions.

In this complex combination of exhaust aftertreatment system; the PM, which is composed of solid and volatile compounds, is affected and undergoes transformations by 
all the individual aftertreatment devices in ways related to the engine operating conditions (e.g. load), as well as thermodynamic and chemical conditions of the devices themselves. As a result, post-2010 US-EPA emissions standards-compliant engines with new aftertreatment technologies tend to produce a higher nucleation mode in comparison to older engines (Biswas, et al., 2008). Ultimately, the emitted PM endures the final transformation as the exhaust plume is diluted and cooled. In this final step, supersaturation conditions can be achieved for the volatile components of the PM, leading to condensation; thereby, strongly influencing the overall chemical and physical characteristics of PM in the plume. The mixing and cooling conditions are related to the aerodynamic field in which the exhaust emission takes place. Faster and more violent mixing events lead to greater chances of supersaturation conditions and formation of smaller particles. Representing an entire flow field is not a simple task, but good indication of the mixing event is the turbulence intensity (TI).

Published literature about PM emissions is largely composed of results obtained in laboratories with few widely accepted techniques: such as CVS, double dilution with ejector diluters, and Particle Measurements Programme (PMP) from the European Joint Resource Council (JRC). In addition, few on-road chase studies describe the fully mixed exhaust with the background air highlighting deficiencies that exist with the CVS approach. Laboratory studies of PM, with CFR-compliant CVS, present a profile of PM in a plume, which is diluted with high-efficiency particulate air (HEPA)-filtered background air - not representative of real-world dilution processes. Atmospheric air comprises PM, often 
referred to as background PM, which indeed interacts with and affects the maturation and growth of particles after being emitted from the tailpipe.

The most important influence can be identified in the smaller particle diameters $(<30 \mathrm{~nm})$, which are generally constituted by the condensation of the volatile compound, called nucleation mode. Gautam et al. (2003) performed the first full-scale wind tunnel plume investigation from a heavy-duty diesel truck (HDDT) and showed that the main mixing event between exhaust stream and the background air occurs within $6 \mathrm{ft}$ from the exhaust stack. On the other hand, the advancements in emission reductions in the last decade demand a new and more detailed analysis. Several research studies have shown and confirmed that DPF-equipped trucks (US-EPA 2007 and newer) emit less PM mass, but the total particle count number could be significantly higher, especially for a predominant nucleation mode in comparison to non-DPF equipped vehicles (Britt \& Ayala, 2002). Several studies have shown that prolonged exposures to HDDE PM emissions can cause finer particles to penetrate deeper into the pulmonary system, with a greater potential of adverse health effects (WHO, 2012).

Since June 2010, several wind tunnels across the US were evaluated by the author, and deemed unsuitable. Two prime criteria were identified: the tunnel had to be an opencircuit configuration and have at least $100 \mathrm{ft}$ downstream of the test vehicle for plume interrogation. The length criterion was based upon work conducted by Gautam et al. (2003) in the NASA Langley Wind Tunnel facility. The wind tunnel at West Virginia University is designed and built to characterize exhaust plumes from heavy-duty diesel vehicles (HDDVs) equipped with and without exhaust aftertreatment systems (Littera, et al., 2012). 
Specifically, the focus of this work is characterize the concentration and size distribution profiles of PM, to further provide valuable information in the assessment of corresponding toxicity (Giechaskiel, et al., 2005) of PM emissions from HDDTs as a function not only of the engine operating conditions, but also as they are strongly influenced by different mixing and cooling processes between the exhaust gas stream and the background air in the maturing exhaust plume. These experiments are fundamentally different than the CVS tunnel or double dilution system (DDS) which use HEPA-filtered air and do not typically provide length scales for realistic, unrestricted plume development and appreciable residence times. At the open loop WVU wind tunnel, unfiltered air is inducted to serve as more realistic background dilution air for the diesel exhaust than a CVS tunnel. Typical background air from the ambient contains a wide spectrum of PM particles, and the accumulation mode particles play an important role in the dilution and transformation of ultrafine particles from the new diesel DPF and SCR technologies.

This work aims to contribute to bridge real-world measurements with advantages of the legacy PM work, pushing forward PM knowledge. This is possible thanks to the developed facilities at WVU for testing HDDTs in controlled conditions with high sampling accuracy (typical of laboratory standards), which allow for the capturing of emissions under real-world conditions (typical of on-road chase studies). The plume analysis and the related dilution history will help to better tune the methodology for PM measurements in laboratories to adhere closely to real-world conditions. The results of this study are essential to provide a clearer understanding of PM formation and evolution, in order to further refine models for predicting toxicity of mobile sources of PM emissions 
from current and legacy HDDT fleets; hence, help engine manufacturers and regulatory agencies in their quest for cleaner engines, and more effective clean air policies.

The motivation that drives this work is to investigate the differences between PM emissions measured in laboratories and real world emissions, extending and deepening the work by Gautam et al. (2001) in the NASA Langley. This could help bridging the results from CFR compliant laboratories to real world conditions. To characterize the PM nanoparticles emissions not only to engine points and aftertreatment configurations, but also to mixing and cooling conditions with the background air. In addition, to develop in WVU testing capabilities to characterize real world exhaust plume formation in a laboratory setting.

With those drivers in mind the main objective of this work is to correlate and compare PM measurement in plumes generated by different US-EPA compliant HDDVs at different vehicle/wind speed with common laboratory measurement techniques. In addition, the main objective involves the investigation of the effect of the turbulence on nanoparticles formation. 


\section{Chapter 2: Literature Review}

\subsection{PM ANd Related Health EFFECT}

PM emissions from diesel engines are a threat to human health and the environment, as indicated by numerous epidemiological studies, leading to increased mortality (Englert, 2004) (Pope, 2007); hence putting the population living adjacent to major roadways and in densely populated urban areas at an elevated risk. It is also shown, by long term studies, that health effects are inversely related to the distance people live from major highways (Brauer, et al., 2002) (Brunekreef, et al., 1997) (Finkelstein, 2003). The National Institute for Occupational Safety and Health (NIOSH) and the USEPA have characterized diesel exhaust as a potential human carcinogen, while the International Agency for Research on Cancer (IARC), which is part of the World Health Organization (WHO), classified it "as carcinogenic to humans" due to a substantial body of evidence that links exposure to diesel exhaust with elevated risk for lung cancer (WHO, 2012).

Although, the WHO report is based on studies where PM emissions do not completely represent present-day diesel engine values (Silverman, et al., 2011). In addition, a multitude of studies showed inflammatory responses or alterations in immune responses in cases of controlled human exposure to diesel PM (Nightingale, et al., 2000) (Seagrave JC, 2001) (Seagrave, et al., 2002). Ultrafine particles (UFPs), in contrast to larger-sized particles, pass into the bloodstream by different transfer routes and mechanisms and then they are distributed into other body organs, including the brain with potential neurotoxic

effects. The call to reduce health problems leads regulating agencies and manufacturers to develop technological improvements aimed at the reduction of toxic emissions (Marchwinska-Wyrwal, Dziubanek, \& Hajok, 2011) (Alfoldy, Giechaskiel, Hofmann, \& 
Drossinos, 2009). However, the epidemiological pattern for UFPs is still less evident when compared to larger particles (Gilmour, et al., 2007) and requires further research.

\subsection{PM COMPOSITION AND DESCRIPTING PARAMETERS}

The diesel PM (DPM) emitted by HDDEs is a rather complex entity, mostly composed of solid compounds (soot or elemental carbon, ash), volatile compounds (organic carbon) and sulfur compounds (see Figure 2-1). The USEPA defines it as "the mass collected on a filter from exhaust that has been diluted and cooled to $52^{\circ} \mathrm{C}$ or below" (SAE, 1993). Solid carbon is formed during combustion in locally rich regions; much of it is subsequently oxidized. This residue is exhausted in the form of solid agglomerates (Kittlelson, 1998) (Burtscher, 2001). A tiny fraction of the atomized fuel and evaporated lube oil escapes oxidation and appears as volatile or soluble organic compounds (generally described as the soluble organic fraction, SOF) in the exhaust. The SOF contains polycyclic aromatic compounds containing oxygen, nitrogen, and sulfur (Kittlelson, 1998) (Burtscher, 2001). Most of the sulfur in the fuel is oxidized to $\mathrm{SO}_{2}$, but a small fraction is oxidized to $\mathrm{SO}_{3}$, which leads to sulfuric acid and sulfates in the exhaust particles.

The adoption of ultra-low sulfur diesel (ULSD) (sulfur content <15ppm) as standard on-road diesel fuel for the USA drastically reduced sulfur emissions. The production of $\mathrm{H}_{2} \mathrm{SO}_{4}$ (sulfuric acid) was not completely eliminated it, due to the presence of sulfur in lube oil (Rönkkö, et al., 2006). Metal compounds in the fuel and lube oil lead to a small amount of inorganic ash. Figure 2-2 shows typical composition of the PM for a HDDE tested using the U.S. Heavy-Duty Transient Test (HDTT) (Kittlelson, 1998). The sulfuric acid/sulfate fraction is roughly proportional to the fuel sulfur content. The fraction associated with unburned fuel and lube oil (SOF) varies with engine design and operating conditions (Kittlelson, 1998). It can range from less than $10 \%$ to more than $90 \%$ by mass. 


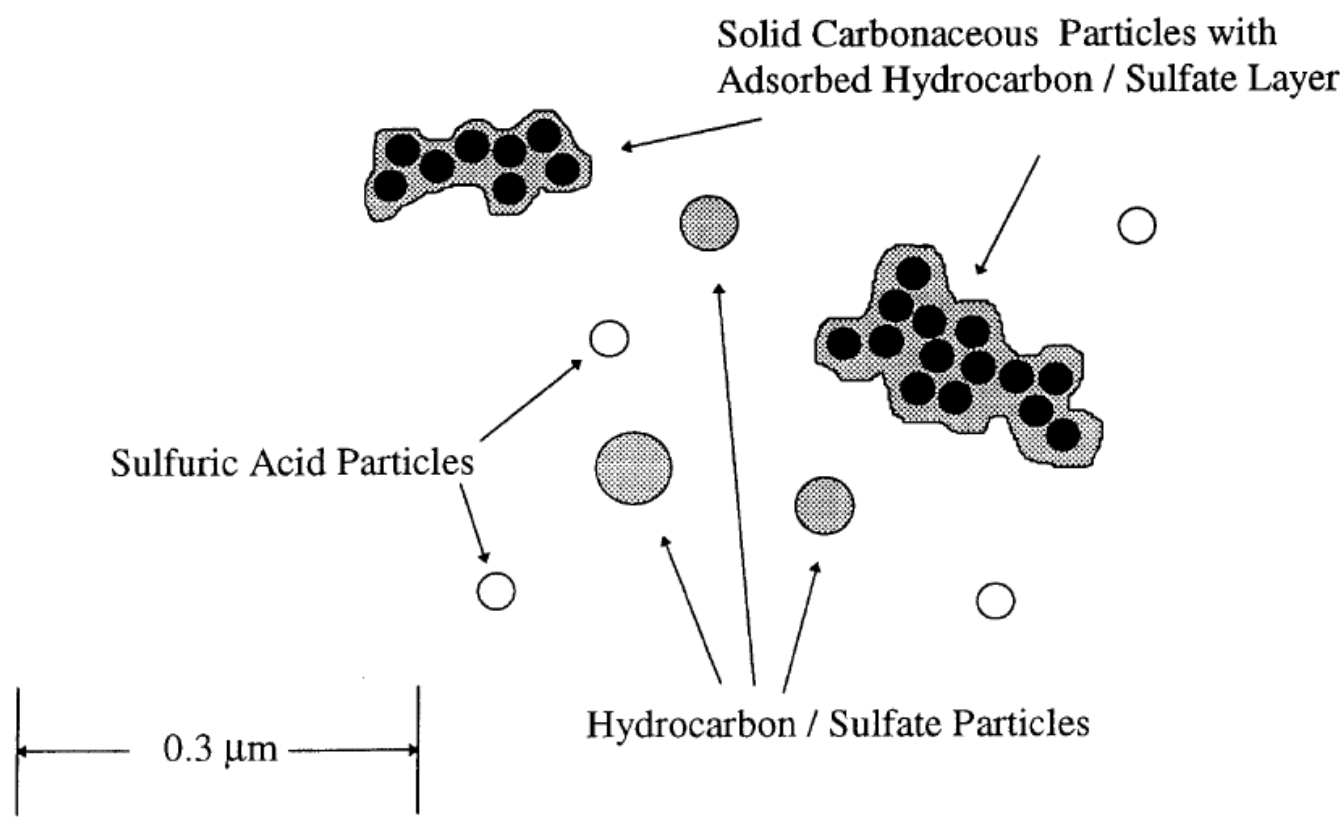

Figure 2-1: Typical composition and structure of engine exhaust particles

(Kittlelson, 1998)

The composition of exhaust particles depends upon where and how they are collected. As the exhaust is diluted and cooled; nucleation, condensation, and adsorption transform SOF to solid and liquid PM. High temperatures in the tailpipe maintain most of the volatile compound in the vapor and gas phases. The details of the dilution and cooling processes determine the relative amounts of material that adsorb or condense onto existing particles or nucleate to form new particles (Kittlelson, 1998) (Burtscher, 2001). 


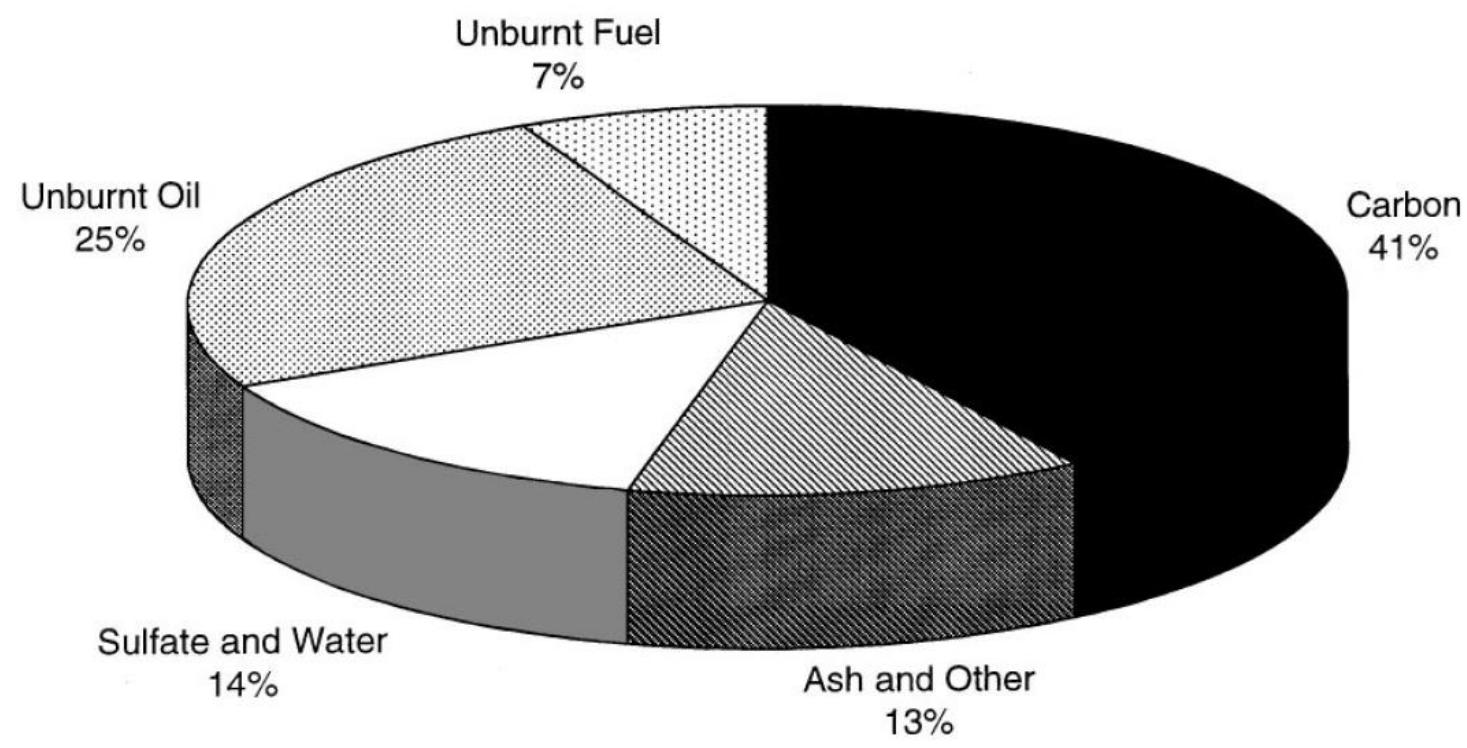

Figure 2-2: Typical particle composition for a HDDE tested in a heavy-duty transient cycle (Kittlelson, 1998)

The DPM presents a wide range of particle dimensions, or diameters, that span from $5 \mathrm{~nm}$ to $1000 \mu \mathrm{m}$ (older engines without any aftertreatment devices), or from $5 \mathrm{~nm}$ to $100 \mathrm{~nm}$ (newer engines with aftertreatment systems). The DPM, in its most general form, shows a triple mode particle size distribution and concentration, depending on several factors, which can be seen in Figure 2-3. The presence of aftertreatment systems, dilution conditions, and engine operation points affect the PM (Britt \& Ayala, 2002). In most cases, two modes are present; the one that covers the larger particles is called accumulation mode (50nm to $1000 \mathrm{~nm})$, while the second is called nucleation mode. The separation between these two modes is in proximity of $30 \mathrm{~nm}-50 \mathrm{~nm}$. The coarse mode is considered negligible for newer engines. 


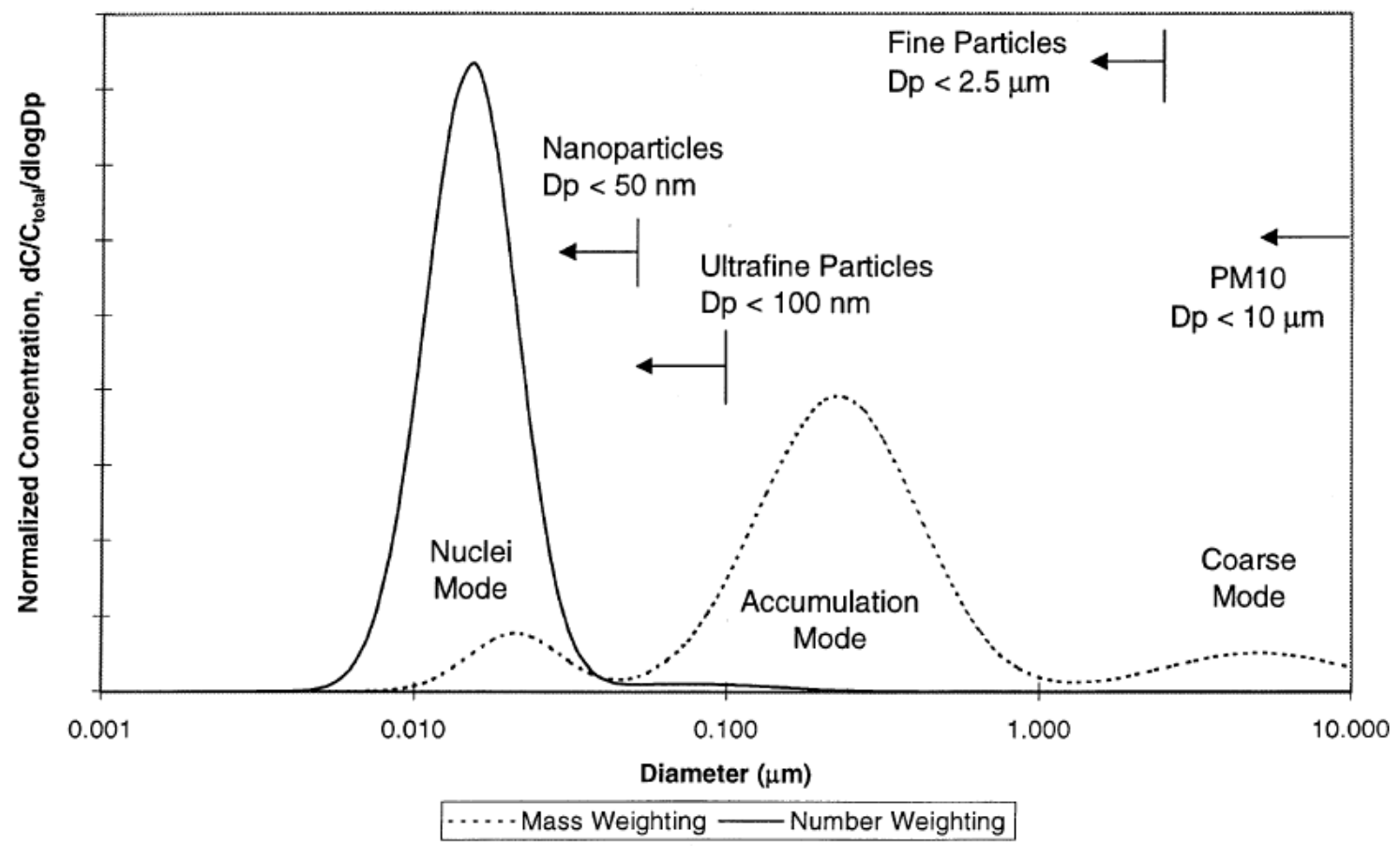

Figure 2-3: Typical engine exhaust size distribution both mass and number weightings are shown (Kittlelson, 1998).

In case of a DPM double mode particle size distribution and concentration, it is widely documented that the accumulation mode is generally composed of the carbonaceous agglomerates (solid compound) of the DPM, which account for the majority of PM mass, while the nucleation mode composition is predominantly composed of the volatile compounds which nucleate during the dilution with background air (Littera, et al., 2011), (Biswas, et al., 2008), and (Biswas, Verma, Schauer, \& Sioutas, 2009). The solid particles that compose the accumulation mode tend to not be heavily affected by dilution and cooling conditions, even if they act as a base for the condensation of the much smaller nuclei (Rönkkö, et al., 2006). The nucleation mode typically contains 1-20\% of the particle mass and more than $90 \%$ of the particle number (Kittlelson, 1998) (Burtscher, 2001). 
As mentioned earlier, the nucleation mode in DPM particle size distribution and concentration (generally $<30 \mathrm{~nm}$ ) is determined by the cooling and dilution processes that the exhaust undergoes, mainly composed of vapor phase substances (SOF) present in the exhaust, which tend to condense and nucleate. The turbulent and fast interaction of the exhaust and the background air lead to vapor phase of the exhaust to supersaturation conditions. Once those conditions are reached in the early stages of the plume, the nucleation mode commences.

Based on the classical theory of homogenous nucleation, clusters form in proximity of the saturation conditions, with a dynamic equilibrium of losing and gaining molecules from the two phases (Oxtoby, 1992). At a given temperature, the supersaturation of vapor occurs and clusters present a positive growth rate. Because cluster growth beyond the critical nucleus is fast, the rate at which clusters emerge is close to the rate of formation of critical nuclei (Oxtoby, 1992). The nucleation rate changes so rapidly with supersaturation, it is very small (almost unobservable) for conditions right below the critical supersaturation conditions, and very large once the critical conditions are achieved (Oxtoby, 1992).

The nucleation mode is also generated through heterogeneous nucleation, where solid particles in the range of nanometers, act as a cluster where the vapor phase can condensate. These solid nanoparticles generally are metallic ash, small carbonaceous nuclei, or even sulfates. The interaction between the fluid particles and the condensation nuclei tends to lower the barrier to nucleation, and critical nuclei form at a much faster pace (Talanquer, 2002).

Homogeneous nucleation of volatile matter to form nanoparticles becomes more likely when the concentration of carbonaceous agglomerates is reduced by aftertreatment or cleaner combustion processes (Kittelson \& Abdul-Khalek, Formation of Nanoparticles during Exhaust Dilution, 1999) (Burtscher, 2001). During supersaturation conditions, the 
most important parameter is the saturation ratio, which is evaluated as the ratio of partial pressure to vapor pressure. Figure 2-4 shows that the presence of solid carbonaceous agglomerates (soot) in the exhaust can reduce the saturation ratio of condensable species (Kittelson \& Abdul-Khalek, 1999). The carbonaceous agglomerates may be thought of as sponges that soak up volatile particle precursors and prevent them from reaching the level of super-saturation necessary to produce nucleation (Kittelson \& Abdul-Khalek, 1999).

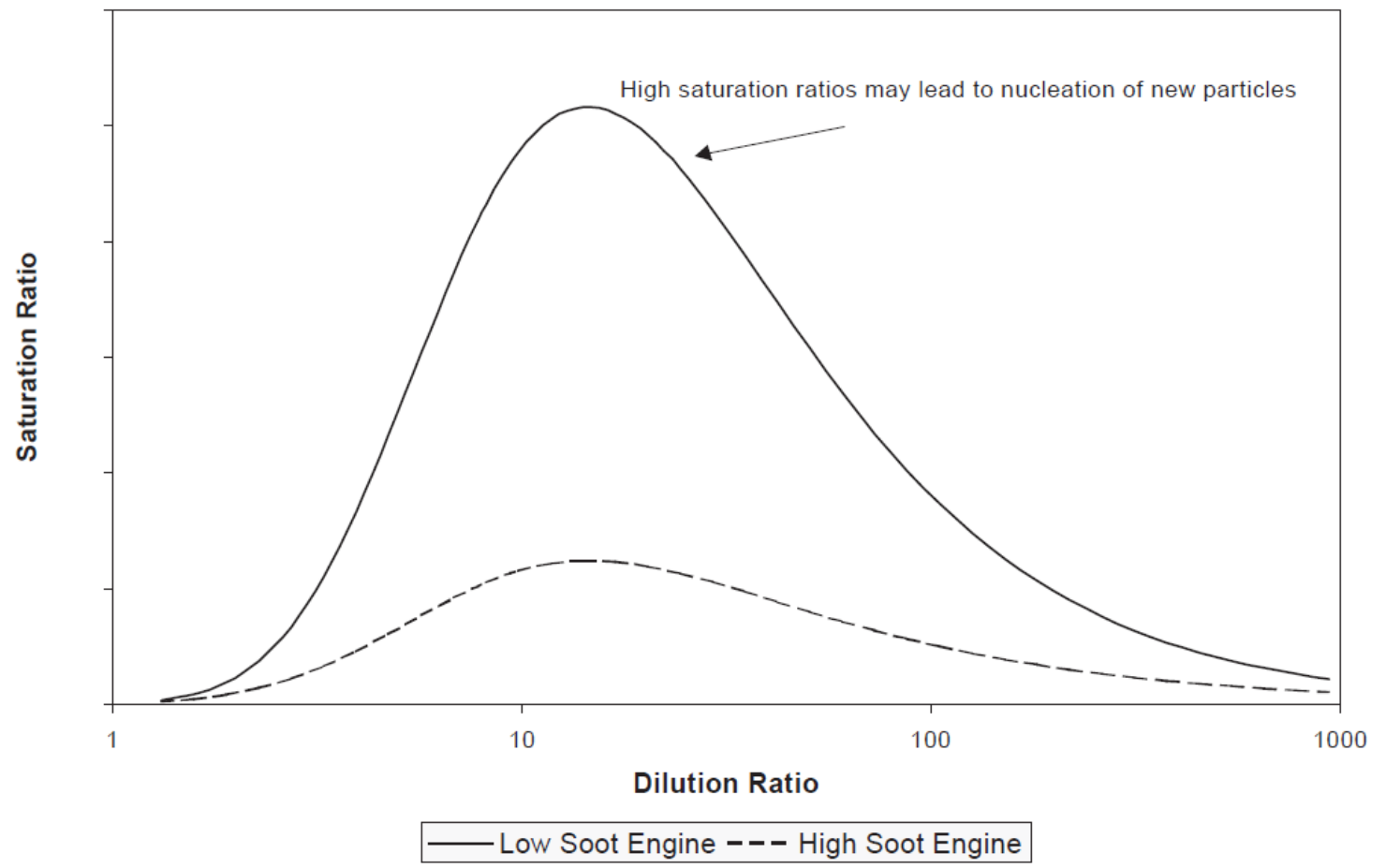

Figure 2-4: Influence of solid particles on saturation ratio during adiabatic dilution (Kittelson \& Abdul-Khalek, Formation of Nanoparticles during Exhaust Dilution, 1999)

Thus, carbon (soot or carbonaceous agglomerates) in the exhaust will tend to suppress the formation and growth of nanoparticles. The potential of an engine to form nanoparticles will depend upon the relative concentrations of volatile particle precursors 
(sulfuric acid and hydrocarbons) and solid carbon (Kittelson \& Abdul-Khalek, 1999) (Burtscher, 2001). Consequently, nanoparticle formation may be more likely with clean, low-carbon emission engines, especially when the concentration of carbon has been reduced more markedly than that of volatile particle precursors (Kittelson \& AbdulKhalek, 1999).

\subsection{PM InTERACTION With DiLUTION AIR}

The strongest cooling and mixing of the exhaust is observed at the exhaust stack outlet and in its proximity, leading to more favorable supersaturation conditions. This is mainly related to the wake generated by the exhaust stack itself and the highest concentration gradient present in the plume between the raw exhaust and the background air (Littera D. , et al., 2013) (Kim, Gautam, \& and Gera, 2001). The rapid cooling and mixing occurring in the early stage is defined by strong turbulence intensity (TI), which is the measure of the turbulent mixing occurring in a stream. Studies by Gautam et al. (2003) and Rönkkö et al. (2006) confirmed that nucleation mode formation takes place in the initial stages of plume formation. Indeed the Rönkkö et al. (2006) on-road chase study does not differentiate in PM emissions 5 meters after the exhaust stack. Indeed, it is found that the number of small particles decreased to a greater extent than did the number of large particles with increasing distance from emission point, suggesting coagulation played a dominant role in the evolution of the size distribution. Because the evolution of particle size and mixing state starts at the point of emission and proceeds rapidly, it is important to examine the evolution near the source at high spatial and temporal resolution (Jacobson \& Seinfeld, 2004).

This is especially true for urban to global scale atmospheric modeling since this evolution invariably occurs at a spatial scale smaller than that of the intrinsic model grid 
scale, and the evolved distribution should be implemented into the model (Jacobson \& Seinfeld, 2004). In addition, the coagulation of nanoparticles is strongly affected by the background PM, heterocoagulation of emitted soot with background particles produced new mixtures in increasing concentration with distance from the emission source (Jacobson \& Seinfeld, 2004), and these phenomena cover a time scale from tenths of a second to tenths of a minute. Measurements of PM from the plume with set distances can be parameterized with time, generally defined as "residence time." Figure 2-5 shows the results of the simulations performed by Jacobson, et al. (Evolution of Nanoparticle Size and Mixing state near the Point of Emission, 2004) of nanoparticles coagulation processes, replicating the atmospheric conditions of the Los Angeles area in a clear summer day. Figure 2-5 (a) shows the evolution of the sum of 10 size distributions over time. The figure indicates that the first peak is negligible in favor of the second peak of 40nm after 30-45 minutes. The 8-s distribution in Figure 2-5 (a) is somewhat similar to Fig. 4a of Zhu et al. (2002). However, Fig. 4d of Zhu et al. (Study of Ultrafine Particles Near a Major Highway with with Heavy-duty Diesel Traffic, 2002) shows that their first peak (from their Fig. 4a) disappeared in favor of the second peak of 40-50nm in only 1-2 minutes. Zhu et al. (2002) hypothesized that, because the number of small particles decreased significantly while the number of large particles did not, Brownian coagulation played a major role in the evolution of the nanoparticle size distribution. Based on the calculation in Figure 2-5 (a) here, it appears that Brownian coagulation alone may not be fast enough to account for the evolution of the size distribution found in Zhu et al. (2002). Kittelson (2002) results fall between the aforementioned time frame, indicating that a $10 \mathrm{~nm}$ particle presents a life of less than 15 minutes. 

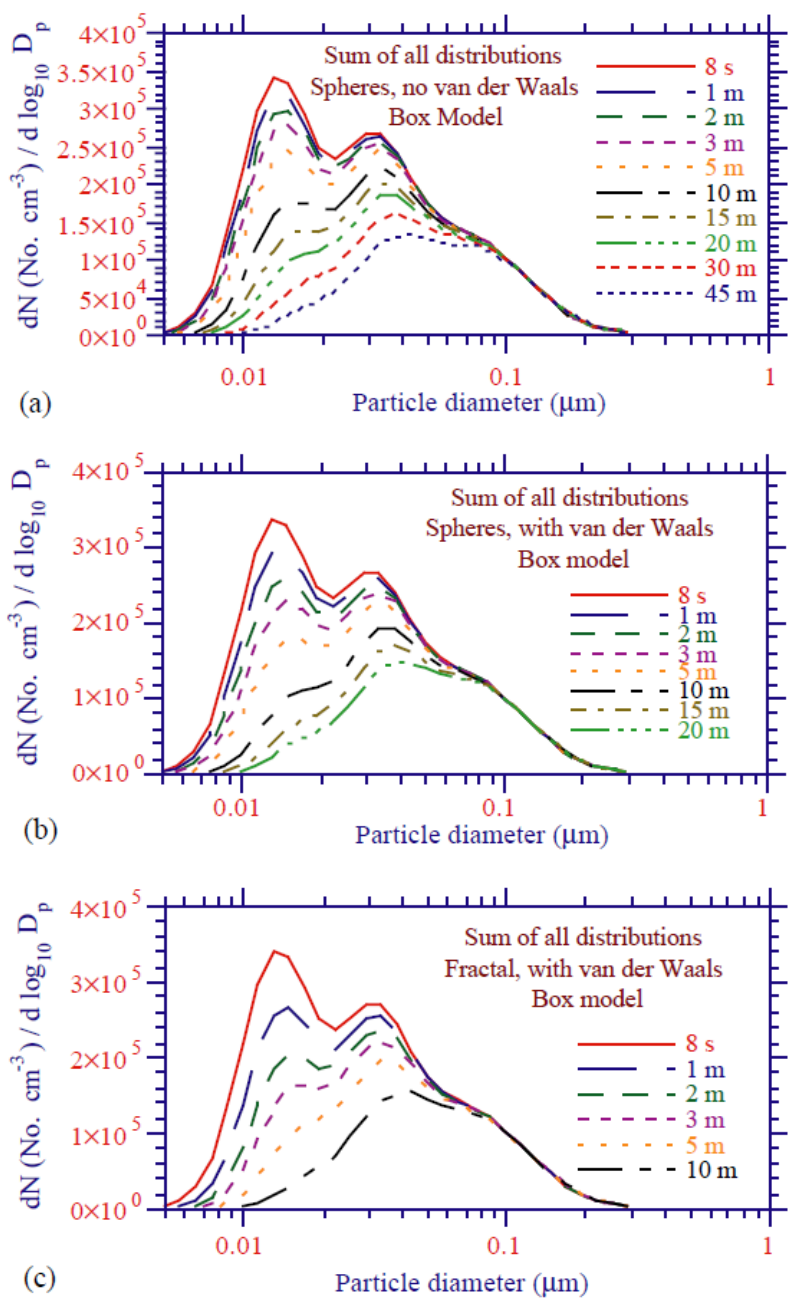

Figure 2-5: Box-model calculation of the evolution of the sum of 10 aerosol distributions by coagulation when (a) particles are assumed spherical and no van der Waals or viscous forces are acting, (b) particles are assumed spherical and van der Waals and viscous forces are acting, and (c) particles are assumed fractal and Van der Waals and viscous forces are acting (Jacobson \& Seinfeld, 2004).

Figure 2-5 (b) and (c) show the effect of van der Waals/viscous forces and fractal geometry on the size distribution of aerosols simulated in Figure 2-5 (a). Figure 2-5 (b) 
shows that treatment of van der Waals/viscous forces but no fractal geometry decreased the time of removal of the first peak from 30 to 45 minutes to about 20 minutes. Figure 25 (c) shows that further accounting for fractal geometry decreased the time to about 10 minutes. The analysis here suggests that the inclusion of van der Waals/viscous forces and fractal geometry may account for a greater share of the evolution of nanoparticle size than Brownian motion alone.

Dilution was found to be more important than coagulation at reducing the total number concentration of particles near the source of emission, but the relative importance of dilution versus coagulation depends on concentration near the emission source (Jacobson \& Seinfeld, 2004). Self-coagulation of emitted soot caused over an order of magnitude more particle loss than did heterocoagulation in the first few minutes after emission (Jacobson \& Seinfeld, 2004). Although, the PM particle size distribution and concentration considered by Jacobson, et al. (2004) is a pre-2004 USEPA on road emissions regulation, with no control devices for the PM. Vehicles tested by of Jacobson, et al. (2004) are considered older engines without DPFs, with mass soot emissions two order of magnitude higher than newer DPF-equipped HDDEs (Littera, et al., 2011); this greatly reducing the chance for self-coagulation.

An important remark that can be drawn by Jacobson, et al. (2004) is that if coagulation is present, no evidence of substantial coagulation or coalescence phenomena within eight seconds from the emission is found. This indicates that the investigation of the formation of the nucleation mode needs to focus in the first few seconds after the emissions in background air $(<8 s)$.

Yu et al. (2004) showed that combustion processes produce naturally charged PM particles in the exhaust. Yu et al. (2004) indicated that the presence of chemiions in the exhaust generally lead to a higher chance of producing nucleation mode. On the other hand, 
the same work shows that the concentration of chemiions in the exhaust decrease rapidly due to the electrical conductivity of the exhaust transfer pipes. This drop in chemiions is evaluated to be of three orders of magnitude within one second of residence time in the exhaust transfer lines.

\subsection{EfFect of Aftertreatment Components on PM emissions}

The already intricate scenario of PM emissions for ultra-fine particles (UFPs) $(<100$ nm) was further complicated by the introduction of Diesel Particulate Filters (DPFs), initially as a mandatory retrofit technology in the state of California and eventually a requirement at a federal level. DOCs remove mainly organic compounds, with a reduction in the formation of nucleation mode particles (Kittlelson, 1998). DPFs efficiently reduce PM mass-based emissions, removing nearly all solid soot particles in the accumulation mode $(0.05 \mu \mathrm{m}$ to $1 \mu \mathrm{m}$, diameter), and they exhibit PM removal efficiencies in excess of 99\% for both new and aged DPFs (Littera, et al., 2011).

On the other hand, the removal of the solid compound (black carbon) produces a significant increase of particles in the nucleation mode region. Thus, newer DPF-equipped HDDEs show remarkable nucleation modes if compared to non-equipped ones (Ardanese, et al., 2009). This result is related to the capacity of solid particles to absorb the volatile compounds and prevent them from nucleation. Once removed, the vapor phase remains free in the treated exhaust, with greater chance to nucleate (Ardanese, et al., 2009). The same results are confirmed by Biswas et al. (2008) indicating that under medium loads and cruise conditions USEPA 2010 compliant HDDEs show a remarkable nucleation mode formation.

Focus on the PM emissions of DPF-equipped trucks is necessary, since all HDDEs compliant with present on-road US-EPA regulation are equipped with DPFs. Rönkkö et al. 
(Effect of Dilution Conditions and Driving Parameters on Nucleation Mode Particles in Diesel Exhaust: Laboratory and On-road Study, 2006) showed how the nucleation mode composition at low engine loads is dominated by unburned fuel and hydrocarbons $(\mathrm{HC})$, due to low temperature and low efficiency combustion. At higher loads, the fuel sulfur content and the formation of $\mathrm{SO}_{3}$ compounds in the DOC are the leading factors for the nucleation mode. It is important to remember that fuel with $250 \mathrm{ppm}$ sulfur content was used during the Rönkkö et al. (Effect of Dilution Conditions and Driving Parameters on Nucleation Mode Particles in Diesel Exhaust: Laboratory and On-road Study, 2006) study. Ultra-low Sulfur Diesel (ULSD,) as an on-road fuel standard $(<15 \mathrm{ppm})$ for the USA, significantly reduced $\mathrm{SO}_{3}$ and $\mathrm{H}_{2} \mathrm{SO}_{4}$ in the exhaust.

In addition, starting from 2010, urea-based SCR has been chosen by most of the HDDE manufacturers as the most affordable and reliable way to reduce $\mathrm{NO}_{\mathrm{x}}$ emissions as required by the on-road USEPA 2010 regulation, which indeed complicated the PM composition and evolution. SCR requires ammonia-based reductant or diesel exhaust fluid (DEF) to reduce $\mathrm{NO}_{x}$ emission, suggesting a possible influence onto PM. The literature failed to thoroughly investigate the effect of the SCR onto PM, but the presence of ammonium in nucleation modes was identified, proposing ternary nucleation as a possible mechanism for particle formation (Biswas, Verma, Schauer, \& Sioutas, 2009).

\subsection{PM MEASUREMENTS IN LABORATORY AND ON-ROAD CONDITIONS}

Most of the advancement in emissions reduction over the last decade in diesel engines has been achieved with the aid of DOCs, DPFs, SCRs, and advanced combustion technologies. Although, these achievements in emissions measurements have been performed in laboratories generally equipped with a CVS or dilution strategies from raw exhaust as regulated by the CFR. 
Fewer on-road chase studies are available in literature describing PM in plume formation, which could provide a valuable insight to PM emissions in real-world conditions. Vehicle chase studies show results of complete diluted exhaust emissions, lacking of the investigation on formation and evolution for the nucleation mode of the PM. The Giechaskiel et al. (2005) chase study faced the difficulty of monitoring the same background conditions, which leads to a lack in standardization when dealing with different vehicles and driving patterns. Several studies indicate that weather conditions present a remarkable effect on PM emissions, such as temperature and humidity. Cooler air temperatures lead to faster cooling and a subsequent distinct nucleation mode (Casati, Volker, Vogt, \& Benter, 2007) (Kittelson, et al., 2000). Variations of $30^{\circ} \mathrm{C}$ in background air temperatures lead to a difference in the concentration of the nucleation mode median of three orders of magnitude (Kittelson, et al., 2000). Higher background air relative humidity tends to increase the nucleation mode concentrations, but this effect is not constant with background air temperatures (Casati, Volker, Vogt, \& Benter, 2007). The effect of humidity results to be stronger at high background air temperatures $\left(50^{\circ} \mathrm{C}\right)$, where an increase of from $0 \%$ to $13 \%$ in relative humidity generates an increase of one order of magnitude in the nucleation mode concentration (Casati, Volker, Vogt, \& Benter, 2007). Instead, at low background air temperatures $\left(10^{\circ} \mathrm{C}\right)$, the humidity effect is negligible, and no statistical differences are observed for relative humidity values between $0 \%$ and $100 \%$.

On-road measurements and roadway tunnel measurements (later examined) lack in detailing the PM emissions from a single vehicle from the moment of which the raw exhaust leaves the truck, to the moment when there is a complete dilution. Wang et al. (Onroad Emission Factor Distributions of Individual Diesel Vehicles, 2011) proposed a costand time-effective approach to obtaining the emission characteristics of individual on-road trucks and buses, and to obtaining the distribution of the emission factors from this on-road 
population. The susceptibility of the approach to the carbon balance provokes variance of $26-40 \%$ in PM emissions with a $2 \%$ variance of $\mathrm{CO}_{2}$ emissions (Wang, Westerdahl, Wub, Pan, \& Zhang, 2011). This indicates that an inaccuracy in the $\mathrm{CO}_{2}$ baseline period selection is the major source of uncertainty in calculating the PM emissions (Wang, Westerdahl, Wub, Pan, \& Zhang, 2011).

\subsection{PM MEasurements in ROAD-Side StUdies}

Highway road-side studies provide information on how the emitted PM could affect nearby areas. Wind plays a key role in vectoring the PM from the highway to a specific area. Changes in wind conditions have been reported to modify the pattern of total particle number concentrations as a function of distance from a major road (Zhu, Hinds, Kim, Shen, \& Sioutas, 2002). Both wind speed and wind direction affect ultrafine PM emissions, where generally higher wind speed provokes lower PM emissions (Zhu, Hinds, Kim, Shen, \& Sioutas, 2002). This is related to the stronger dilution processes provoked by higher wind speeds, which tend to wash away the ultrafine particles (Zhu, Hinds, Kim, Shen, \& Sioutas, 2002). However, at extremely low wind speeds, it would take a considerably longer time for the wind to carry particles to the sampling station, which gives ultrafine particles more time to coagulate with either themselves or with larger particles, a phenomenon that would decrease the total particle number concentration (Zhu, Hinds, Kim, Shen, \& Sioutas, 2002).

In road-side measurement, another key factor is the sampling distance from the highway itself, which is a good indicator of the exposure to PM emissions for building in the nearby areas of highways. Lower PM emissions are identified with increasing distance from the highway. This phenomena increase is more evident with smaller particles (Zhu, Hinds, Kim, Shen, \& Sioutas, 2002) (Hinds, 1999). The smaller the particle, the greater its diffusion coefficients and Brownian motion (Hinds, 1999). As particle size gets smaller, 
the Kelvin effect becomes more important, allowing molecules to leave the particle's surface by evaporation (Zhu, Hinds, Kim, Shen, \& Sioutas, 2002). In addition, when two small particles collide due to their Brownian motion (coagulate), they form a bigger particle (Zhu, Hinds, Kim, Shen, \& Sioutas, 2002). Thus, coagulation reduces number concentrations and shifts the size distribution to larger sizes (Zhu, Hinds, Kim, Shen, \& Sioutas, 2002).

\subsection{COMPARISON OF PM MEASUREMENT IN LABORATORY AND REAL WORLD}

The main limitation in measuring PM number concentrations and size distributions, and relative toxicity, in a laboratory-type setup with a constant volume sampling (CVS) tunnel, is the PM dependency on dilution and cooling processes. These conditions in the laboratory are not accurately represented as they are in real-world conditions. This discrepancy is particularly pronounced for UFPs $(<100 \mathrm{~nm})$. CVS-based PM measurements allow for standardization, but since the dilution air is cleaned by means of a high efficiency particle air filter (HEPA), provoking measurements drift from real-world conditions. In addition, rigorous control of temperature and humidity do not account for the natural variation on the region and season.

Kulmala et al. (2005) analyzed the rate of growth and coagulation of nanoparticles in four different regions of the world, to determine the effect of background PM and thermo-dynamic conditions of background air on the nucleation mode. During the observed particle formation events, the condensation sink was usually higher in more polluted areas (Kulmala, et al., 2005). The condensation sink indicates the tendency of the newly emitted PM to condensate and coagulate on pre-existing background aerosols (Kulmala, et al., 2005). The highest value of condensation sink $\left(5-7 \cdot 10^{-2} \mathrm{~s}^{-1}\right)$ was encountered in New Delhi, while in the European cities of Athens and Marseille, the condensation sink was 
significantly lower (5-10 fold) (Kulmala, et al., 2005). In non-polluted areas, the values of condensation sink were some 50-100 fold lower than those observed in New Delhi (Kulmala, et al., 2005). Taken into account the high seasonal variability of the ambient aerosol size distributions, the calculated condensation sinks and growth rates as well as estimated condensable vapor concentrations and source rates should be used with caution (Kulmala, et al., 2005). These concerns particularly affect short field campaigns, in which the data set is quite limited (Kulmala, et al., 2005).

New particle formation events cannot be observed unless the source rate of condensable vapors is high enough to induce a sufficiently large growth rate for the smallest nucleation mode particles (Kulmala, et al., 2005). The required source rates of $>10^{7} \mathrm{~cm}^{-3} \mathrm{~s}^{-1}$ in the most polluted environment (New Delhi) are 10-100 times larger than those in the European cities (Athens and Marseille) and roughly four orders of magnitude larger than those for our cleanest environments (Kulmala, et al., 2005). Kulmala et al. (2005) indicate that potential candidates for the condensable vapors responsible for the growth of nucleation mode particles are sulfuric acid and various organic vapors of low volatility.

In the lower troposphere, gaseous sulfuric acid is produced mainly by the reaction of sulfur dioxide $\left(\mathrm{SO}_{2}\right)$ with the $\mathrm{OH}$ radical. The concentration of $\mathrm{SO}_{2}$ varies by more than three orders of magnitude between the very clean and polluted environments, whereas the concentration of the $\mathrm{OH}$ radical depends mainly on the intensity of solar radiation. By combining these parameters together, the source rate for gaseous sulfuric acid might well differ by the required four orders of magnitude between New Delhi and the cleanest environments (Kulmala, et al., 2005). Despite large variations in the vapor source rates, the growth rate of nucleation mode particles did not vary by more than one to two orders of magnitude between the different sites (Kulmala, et al., 2005). The primary reason for this 
is that the large vapor source rates usually appear synchronously with large condensation sinks, the overall effect of which is to balance the condensable vapor concentrations (Kulmala, et al., 2005). The average particle growth rates increased, however, with the level of pollution (Kulmala, et al., 2005). This can be explained by the fact the low particle growth rates cannot be observed in heavily polluted environments due to the effective scavenging of the smallest growing particles by the pre-existing aerosol population (Kulmala, et al., 2005).

Casati et al. (2007) compared the variation in PM emissions between an in-situ diluter and chase studies, while controlling the temperature and humidity content of the dilution air when using the diluter. A comparison between laboratory and chasing results at 50 and $120 \mathrm{~km} / \mathrm{h}$ using fuel and low and high sulfur content (15 and 300ppm), shows respectively that at $120 \mathrm{~km} / \mathrm{h}$, the size distributions were bimodal when the high sulfur fuel was used, while with low sulfur fuel only the soot mode was present (Casati, Volker, Vogt, \& Benter, 2007). At $50 \mathrm{~km} / \mathrm{h}$, there was no nucleation mode even with high sulfur fuel, because the catalyst temperature was too low to convert the fuel sulfur to sulfuric acid (Casati, Volker, Vogt, \& Benter, 2007).

Casati et al. (2007) shows that the nucleation mode measured in the laboratory $\left(\mathrm{DR}=10\right.$, dilution air $\left.\mathrm{T}=20^{\circ} \mathrm{C}, \mathrm{RH}=55 \%\right)$ had a similar emission rate as during chasing $\left(\mathrm{T}=25^{\circ} \mathrm{C}, \mathrm{RH}=55 \%\right.$ ); however, the average mode diameter was smaller (the maximum concentration was at or below $10 \mathrm{~nm}$ instead of $15 \mathrm{~nm}$ ). Casati et al. (2007) suggests that the process of particle growth by condensation was stopped in the laboratory sampling system by secondary dilution before completion. Maricq et al. (2001) provided a detailed and direct comparison of PM emissions using three different methods: local tailpipe diluters, wind tunnel measurements, and CVS dilution tunnels. Maricq et al. (2001) recall that dilution tunnel systems were originally developed to avoid the problem of water 
condensation while measuring PM mass, but it is not necessarily appropriate for particle size and number measurements or for mass measurements at very low emission rates.

Also, low soot emissions from diesel engines equipped with particle filters, the nanoparticle mode, artifact or not, can represent an absolutely small, but relatively large component of the particulate mass (Maricq, Chase, \& Xu, 2001). Maricq et al. (2001) indicates that the sensitive instrumentation that is available for particle number measurements can readily provide misleading information about the number of particles emitted. Maricq et al. (2001) postulate that the tailpipe measurements reported compare favorably with those made in the wind tunnel, but still tailpipe sampling does not represent real-world dilution. The main disadvantage of CVS dilution tunnels and local tailpipe diluters is the generation of artifacts. Maricq et al. (2001) concluded that to reduce artifacts, dilution should be done as close as possible to the tailpipe and minimize the contact between the hot exhaust and material surfaces. Once these are minimized, vehicle PM will generally consist of a 10-30 $\mathrm{nm}$ nucleation mode that is highly variable depending on testing conditions, fuel structure, aftertreatment design, and vehicle history; and a 50-100$\mathrm{nm}$ accumulation soot mode that is relatively robust to test conditions (Maricq, Chase, \& $\mathrm{Xu}, 2001)$.

It is clear that the two opposite conditions of CVS and real-world present substantial differences in measured PM emissions. A contained environment with real-world conditions could pick the advantages of the two approaches. A contained environment guarantees control over fundamental parameters, while mimicking as closely as possible the real-world conditions with real background dilution air. Emissions from traffic in a contained environment, such as a roadway tunnel, always depict a delicate topic where the tunnel ventilation itself is a major control parameter as indicated by the work of El-Fade et al. (2001). At the same time, roadway tunnel measurements can identify important 
information regarding the final mixing state of gaseous and particle phase products, but it will not provide any information on the formation and evolution of the plume itself. AbuAllabam et al. (2002) shows clearly how the final state of PM size distributions is strongly influenced by traffic and ambient conditions, and in all cases a strong nucleation mode prevails in number over the accumulation mode. The aforementioned work (Abu-Allaban \& Coulomb, 2002) was performed in 2002, at a time when virtually no modern aftertreatment technologies were commercially available. This dominant nucleation mode could be further enhanced due to the tendency of DPF and SCR to shift PM size distributions towards nucleation mode particles, as mentioned previously.

On the other hand, a roadway tunnel study provides averaged results, where input parameters cannot be changed but only observed, such as engine load, vehicle speed, and vehicle/engine class. Employing a non-recirculating wind tunnel instead, allows for capturing the first few seconds of plume formation and evolution from a single vehicle, thus making it possible to discern the influences of different aftertreatment technologies onto real-world PM evolution after being released from the exhaust stack.

\subsection{PM MEASUREMENTS IN Wind TUNNEL}

Gautam et al. (2003) performed the first investigation of plume measurement from a diesel truck at the NASA Langley wind tunnel. A wind tunnel provides close control on all the major parameters involved. One vehicle at a time can be evaluated, load and speed are decided a priori, and the plume can be investigated along its entire dimensions. This allows for the evaluation of the formation and the evolution of DPM while having realworld conditions. Gautam et al. (2003) indicated how the majority of the exhaust is completely diluted with the background air within $2 \mathrm{~m}$ from the exhaust stack. Several wind tunnels across the U.S. were evaluated by the author, but they were inevitably deemed 
unsuitable, due either to undesired design and performance characteristics or availability. Two prime wind tunnel characteristics were identified as critical for this study: a) the tunnel needed to be an open-circuit configuration and b) it had to provide for a minimum of $100 \mathrm{ft}$ of sampling zone downstream of the test section for plume interrogation.

The length criterion is based upon computational fluid dynamics (CFD) simulations of the flow field within the wind tunnel, conducted by Kim et al. (2001). Simulations indicate that the main mixing process between the exhaust and ambient air stream occurs within a few feet downstream of the exhaust stack, which is in accordance with actual turbulent flow field measurements as reported by Gautam et al. (2003) in the NASA Langley wind tunnel facility. Results from these studies indicated that $\mathrm{CO}_{2}$ concentration, temperature, and dilution ratio in the plume of a heavy-duty truck emitted in a $55 \mathrm{mph}$ wind speed, stabilized at a distance of approximately $40 \mathrm{ft}$ downstream the vehicle exhaust stack, which is in accordance with the results of Casati et al. (2007) where they indicated no variation of the nucleation mode for distances greater than $45 \mathrm{ft}$ from the vehicle.

The need for an open-circuit wind tunnel, on the other hand, is desired by the aim to simulate exhaust plume dilution conditions as close as possible to what is experienced in real-world operating conditions. Furthermore, an open-circuit tunnel allows for a "continuous replacement" of the dilution air; hence PM and gaseous emissions in the background air will not increase as a function of time, as would be the case for a closedloop tunnel. This ultimately allows for an extended sampling duration in order to extensively characterize the exhaust plume. Neither of the two aforementioned criteria are met by any of the tunnels investigated, thus leading the project team to specifically design and construct a wind tunnel facility capable of accommodating the aforementioned design considerations. 


\section{Chapter 3: Design and Construction of the WVU Non-Recirculating Environmental Subsonic Full Scale Wind Tunnel}

This chapter covers the design process and construction of the WVU environmental subsonic full scale wind tunnel, reflecting the indications of the thesis of proposal aim 1, which include the design, construction and qualification of a specific environmental wind tunnel to satisfy the requirements of this research.

\subsection{Wind TunNel Design}

\subsubsection{Design Constraint and Target}

The stringent objectives for this study are to define the WVU wind tunnel characteristics and specifications, which is an important step in the overall design process. The wind tunnel presents a non-recirculating configuration; this key feature guarantees fresh ambient background air flowing over the vehicle and therefore, constant dilution air composition and conditions for the entire test duration. On the contrary, a recirculatingflow wind tunnel could operate only for a few minutes before the entire tunnel is saturated with exhaust gases, strongly deviating from realistic background air concentrations, and ultimately complicating the measurements with continuous background related corrections. The size of the facility and the topography of the surrounding area would prevent the flow exiting the wind tunnel from re-entering the inlet. The construction site is

placed one mile away from any major roads or industrial plants, and it is assumed to attenuate variations of the background conditions over the test period, as well as prevent from increased PM concentrations due to either passing vehicles or industrial plant operations. Eventually, test results indicates almost constant PM and gaseous emission in 
the background air over the entire test campaign, confirming the initial hypothesis. In addition, no indications of the exhaust blow back were observed.

The WVU wind tunnel is a classic suction tunnel, where the vehicle is located upstream near the inlet, and the air is pulled inwards into the test section and then pulled out through a suction fan at the far end of the tunnel (see Figure 3-1). A small convergent section, as well as a flow straightener and settling chamber, is positioned upstream of the test cell in order to stabilize the flow field entering the test section and reduce the turbulence to designated values and remove any swirl flows.

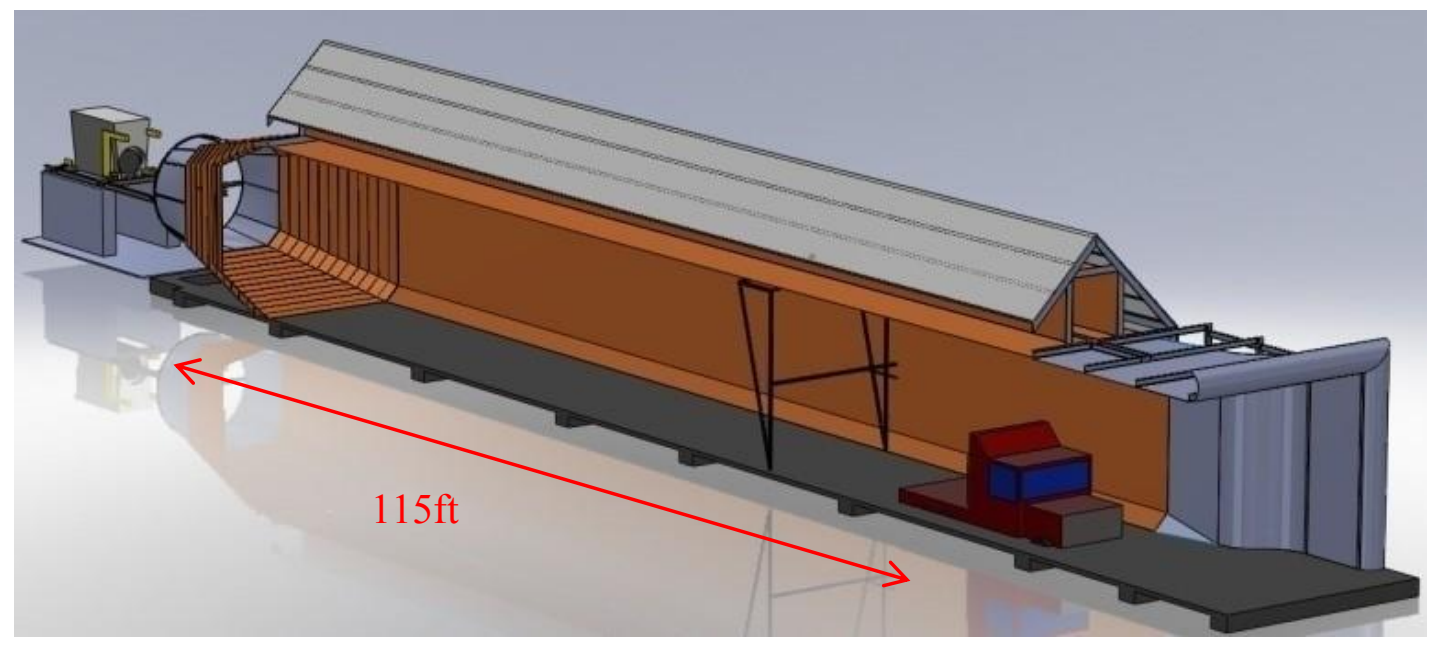

Figure 3-1: CAD cut view representation of the WVU wind tunnel

Finally, a convergent loft surface connects the test cell to the fan section. The suction tunnel design is able to achieve better flow properties and longer sampling distances when compared to a blowing design, since the latter would require a divergent and convergent section (with consequent increase in construction and operational costs) to remove the swirl caused by the upstream fan, as indicated by Rae et al. (1984). Air flow through the wind tunnel is achieved by a five-bladed propeller (10ft diameter carbon fiber blades) with 
adjustable pitch coupled to a large bore diesel engine, capable of providing up to $2200 \mathrm{hp}$. The WVU wind tunnel is a large-scale tunnel, capable of accommodating a full-scale class8 HDDT including its trailer. The full scale WVU wind tunnel solution is suggested to adhere as closely as possible to real-world conditions, since no indications are in present literature regarding the scale model of the vehicle exhaust plume interaction with background air.

As previously noted, the goal of this study is to focus on the dispersing exhaust plume behind the vehicle rather than the aerodynamic forces acting on it, which conventional wind tunnels are typically used for. This important difference is the rationale for the WVU wind tunnel to have a length scale of the test cell much greater than the actual test section. A wind speed in excess of $35 \mathrm{mph}$ is needed for this campaign test with turbulence intensity (TI) less than 1.5\%. Wind tunnel for aerodynamic analysis of airplanes or cars set the $\mathrm{TI}<1 \%$, which is achieved at high cost and energy expenses. The goal of this work to simulate calm air that a truck could encounter on a highway and analyze the plume formation, but not perform an aerodynamic analysis of the truck cabin. The composition of all this requirements, helped to the maximum TI at $1.5 \%$.

Detailed plume analysis requires a system able to sample throughout the entire test cell, without affecting the dynamics of the flow itself. The instrumentation required to characterize the exhaust plume could not be placed within the flow field without strongly influencing it. A single sampling probe, however, could extract a localized fraction of the plume and redirect the sample to the instruments. In order to minimize the length of the transfer line connecting sampling probe and instrumentation, where PM losses occur, a 
novel solution has been adopted by implementing a cart carrying the entire suite of instruments as it moves longitudinally within the ceiling of the tunnel. Underneath the cart, and within the test cell, a gantry system spans the probe vertically and horizontally as depicted in Figure 3- and Figure 3-4. This configuration allows sampling throughout the cross-sectional area of the test cell, and to proficiently move the sampling probe around the truck model placed within tunnel. Position of the probe is monitored with three laser distance meters, capable of 5mm accuracy over the entire wind tunnel test cell. The position accuracy was compared with a separated laser distance meter with accuracy $1 \mathrm{~mm}$.

\subsubsection{Computational Fluid-dynamic Analyses}

With the primary parameters and targets pre-defined, the first step of the design process is to identify the typical plume dimensions for a large range of on-road and offroad vehicles with a roof height of less than $15 \mathrm{ft}$. To that aim, preliminary computational fluid-dynamics (CFD) simulations were performed with the commercially available CosmosFlow $^{\text {TM }}$ software (part of the SolidWorks ${ }^{\mathrm{TM}}$ suite), using a k- $\varepsilon$ closure model. The exhaust gases are modeled by a simple tracer constituent (i.e. $\mathrm{CO}_{2}$ ), which allowed for estimating the interaction and mixing between background air and exhaust stream. For the purpose of defining gross wind tunnel dimensions that allow the exhaust plume to evolve undisturbed, only large-scale flow patterns were taken into account, while small-scale events were disregarded and left for analysis during the actual experimental tests.

Based on that, the tractor model in the preliminary CFD simulations is purposely box-shaped (see Figure 3-2 and Figure 3-3) in order to create a wake larger than a real tractor would. Results indicate that plume growth is very fast in the region closest to the exhaust stack due to the intense turbulent regions developing immediately behind the cabin. 
The plume seems to follow the shape of the aerodynamic wake generated by the tractor. Away from the cabin, the aerodynamics of the vehicle and the associated large scale eddies play a smaller role (once the large scale eddies are broken into smaller ones), and a slow diffusion phenomenon takes place without significantly changing the plume dimensions. Turbulent mixing generated by the tractor's geometry can be identified as the major contributor to the plume formation, evolution, dilution, cooling, and its final shape and size. Results from the aforementioned preliminary CFD simulations further indicate the plume to have an approximate height and width 1.2 times wider than the frontal dimensions of the tractor, therefore being in good agreement with the studies of Kim et al. (2001) and Gautam et al. (2003) where the plume size did not exceed 1.5 times the height of the truck (in case of the worst aerodynamic configuration) at wind speeds representative of highway cruise. Figure 3-2 shows the density contours and streamlines for the low exhaust stack configuration. Since $\mathrm{CO}_{2}$ is used at gas tracer of the exhaust, the variation in density helps to identify the plume.

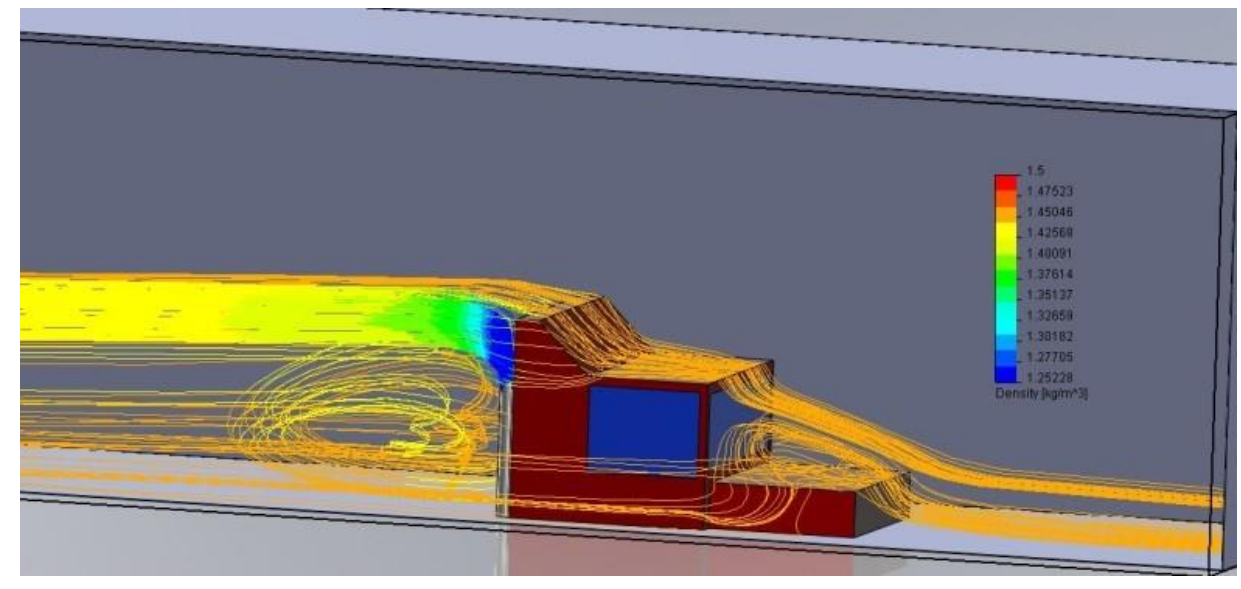

Figure 3-2: CFD simulations, exhaust stack lower than fairing with $35 \mathrm{mph}$ wind speed, density is shown $\left(\mathrm{kg} / \mathrm{m}^{3}\right)$ 


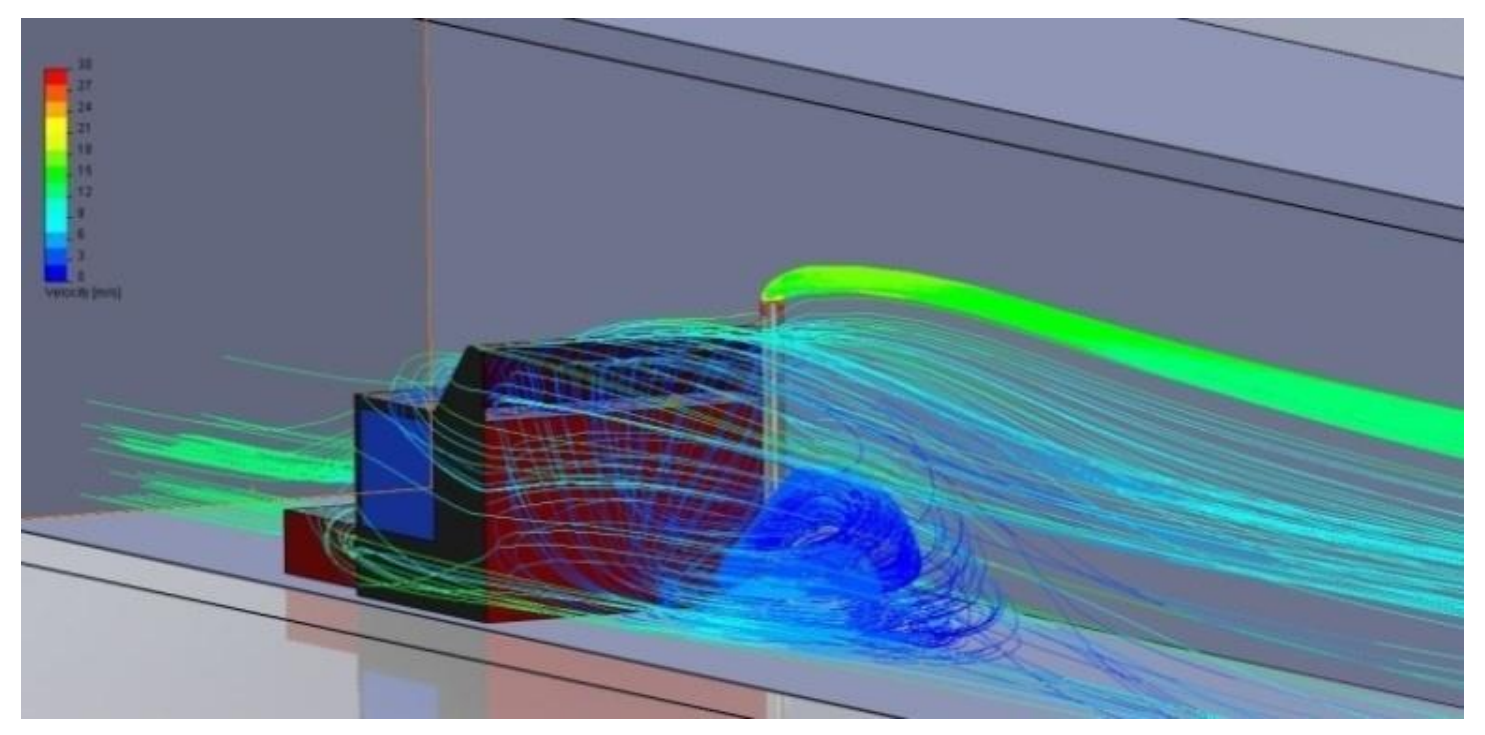

Figure 3-3: CFD simulations, exhaust stack higher than fairing with $35 \mathrm{mph}$ wind speed, total velocity is shown $(\mathrm{m} / \mathrm{s})$

Figure 3-3 shows the velocity contours and streamlines for the high exhaust stack configuration, which help to identify the plume and the wind speed field surrounding the plume itself. Low wind speed are observed in the wake of the truck (close to zero), while higher wind speed are observed in above the fairing (close to $20 \mathrm{~m} / \mathrm{s}$ ), considering an inlet speed of $14 \mathrm{~m} / \mathrm{s}$.

Results shown by Rae et al. (1984) indicate that for a subsonic wind tunnel, the frontal model blockage area needs to be less than $20 \%$ of the total test cross-sectional area. This rule of thumb allows the avoidance of an over-accelerating wind speed in the surroundings of the tractor cabin, since any object in a wind tunnel always acts as a "plug."

Therefore, the cross-sectional area of the test section was defined to be square in shape with a side length of $16 \mathrm{ft}$ (see Figure 3-4), thus ensuring that the tunnel walls would never be in contact with the dispersing exhaust plume, and allowing for a value of the blockage area below $20 \%$ of the total test cross-sectional area. The test cell length was 
defined to be $115 \mathrm{ft}$., which allows for a two second residence time at $35 \mathrm{mph}$, a value well within the time scale for plume formation and initial aging. Rae et al. (1984) further show that in the case of square shaped wind tunnels, the boundary layer (BL) grows faster at the corners due to the interaction between the individual boundary layers developing over each side of the tunnel. In order to homogenize the growth of the boundary layers, and at the same time obtain an additional structural member, a $45^{\circ}$ gusset is placed along each of the four longitudinal corners. It is appropriate for a wind tunnel to be non-interfering with the plume, thus there must be no direct contact (non-wet walls), and also the BL growth should not influence the plume evolution.

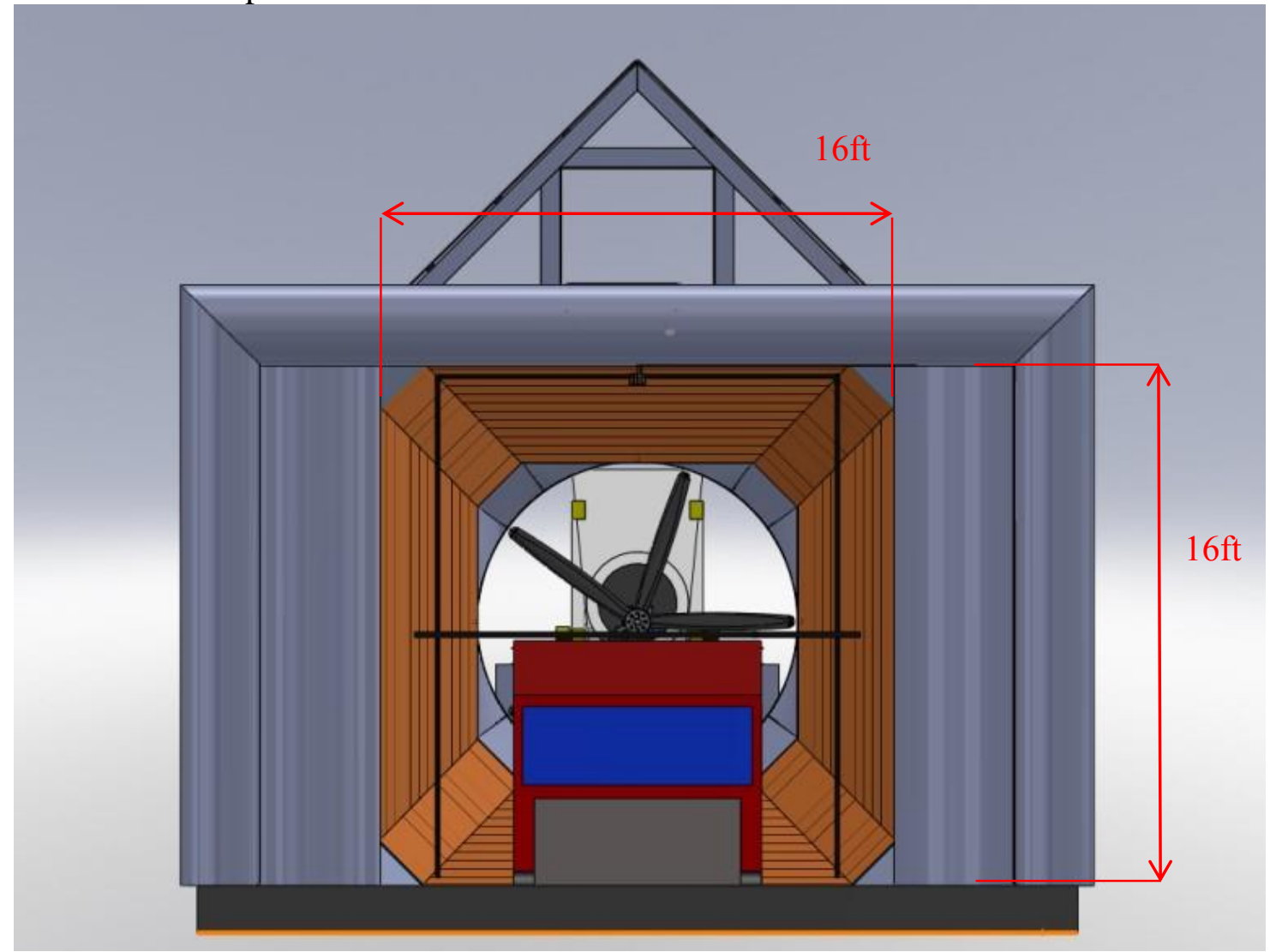

Figure 3-4: WVU wind tunnel entrance section and relative test cell cross section dimensions 
More detailed CFD analyses performed by S. Hamburg in WVU confirmed the previous results. Moreover, they indicated that the dimensions chosen for the wind tunnel suite a HDDT and fulfill the requirements set forth. Simulations are performed on Fluent ${ }^{\circledR}$ with large eddy simulation methods (LES). Figure 3-5 and Figure 3-6 summarize the results from these finer analyses.

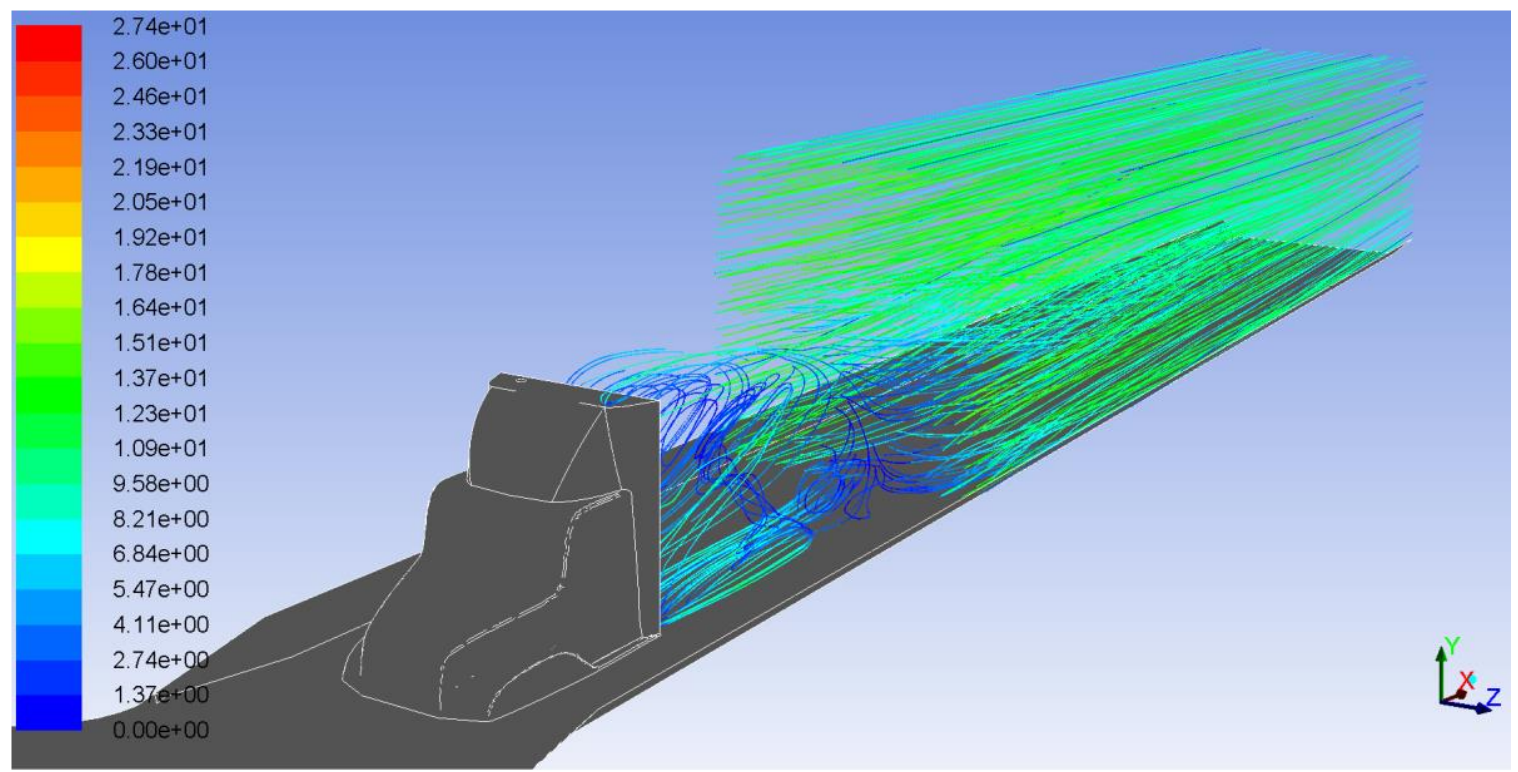

Figure 3-5: Path lines colored by velocity magnitude $(\mathrm{m} / \mathrm{s})$. The Results of the simulation performed by S. Hamburg. 


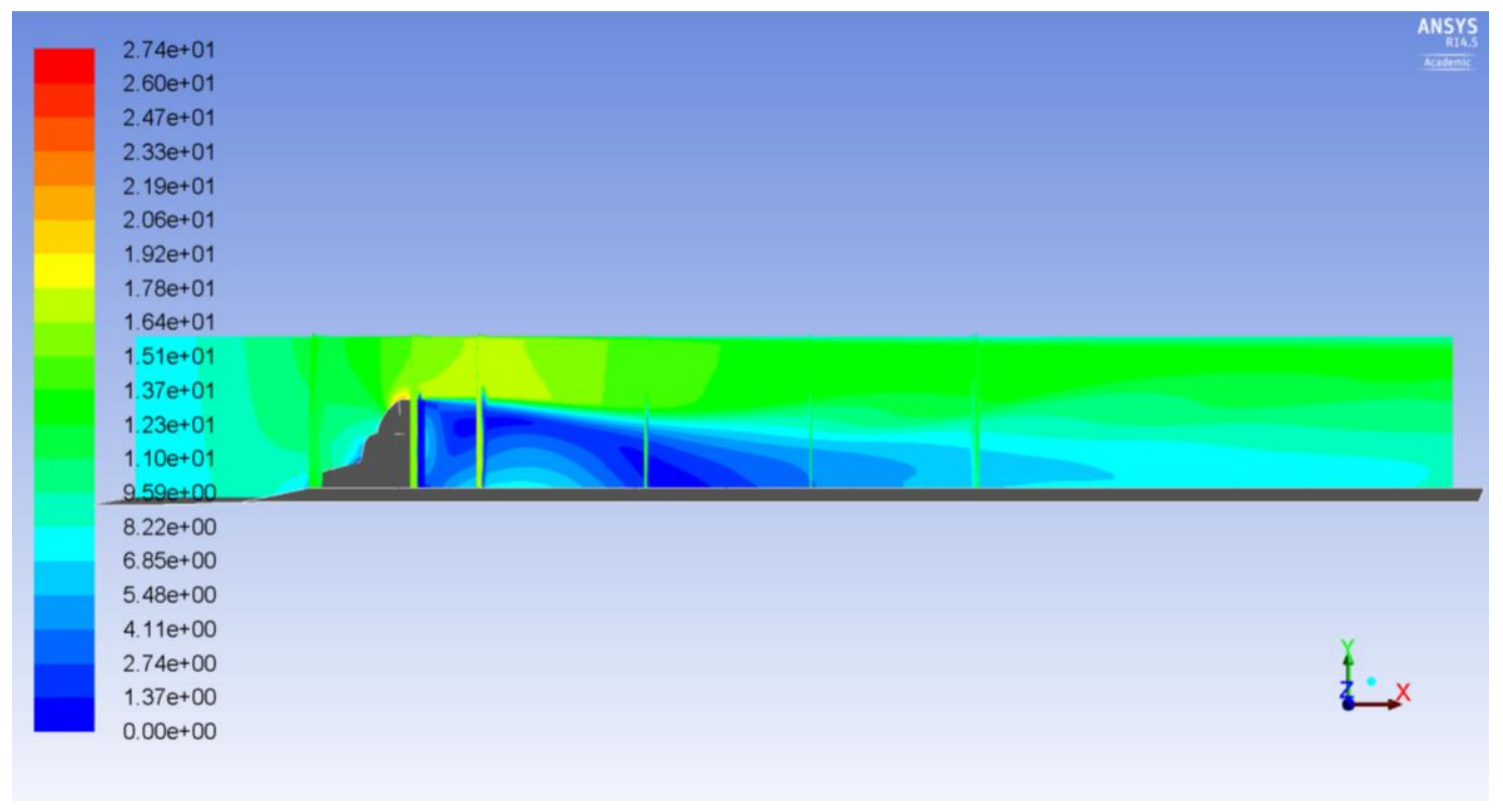

Figure 3-6 Side view of the velocity contours on the tunnel centerline $(\mathrm{m} / \mathrm{s})$. The simulated wind speed is $35 \mathrm{mph}$. Results of the simulation performed by S. Hamburg 


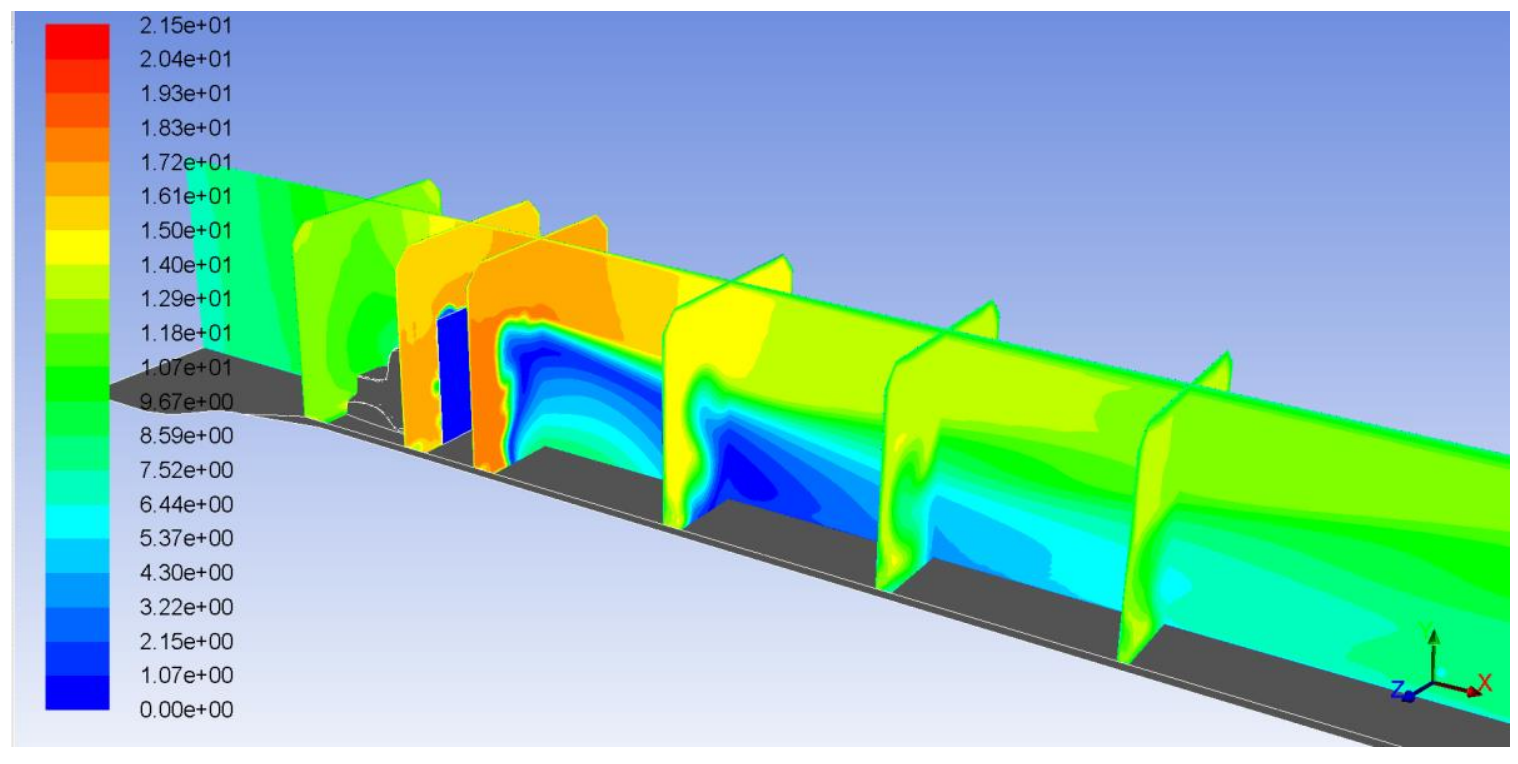

Figure 3-7: Velocity contours on the tunnel centerline, $(\mathrm{m} / \mathrm{s})$ Cross sections are at the point of tunnel contraction and $5 \mathrm{~m}, 10 \mathrm{~m}, 15 \mathrm{~m}$, and $20 \mathrm{~m}$ downstream with one additional at the back of the cab. Results of the simulation performed by S. Hamburg. 
The development of the boundary layer in the entrance length of a "washy" wind tunnel continuously modifies the velocity profile, thus, provoking an increase in bulk velocity in the region outside the boundary layer. This leads to a decrement in static pressure and formation of a longitudinal differential pressure with consequent floating forces acting on any object in the same direction. Rae et al. (1984) indicate that one of the major problems in "washy" wind tunnels is the influence of this longitudinal differential pressure. A common solution is to diverge the test section of the wind tunnel by an angle such that a compensation of the displacement thickness (DT) growth is achieved. The DT is defined as the theoretical distance by which a surface has to be moved parallel to itself (towards the reference plane) in order to yield the same mass flow rate for an inviscid fluid as would be realized by a viscid fluid in the original geometry (Rae, William, \& Pope, 1984).

Since the WVU wind tunnel presents turbulent conditions, even for small wind speeds, the boundary layer development was analyzed referring to the expressions usually employed in cases of turbulent duct flows (White, 1991).

$$
\begin{array}{ll}
\delta_{x}=\frac{0.166 x}{\sqrt[7]{R e}} & \text { Equation 3-1 } \\
\delta_{x}^{*}=\frac{1.728 x}{\sqrt{R e}} & \text { Equation 3-2 } \\
L=\frac{0.1 R e}{D q} & \text { Equation 3-3 }
\end{array}
$$


Where:

$$
\begin{aligned}
& \delta_{x}=\text { Boundary Layer Thickness } \\
& \delta_{x}^{*}=\text { Displacement Thickness } \\
& x=\text { longitudinal coordinate } \\
& L=\text { Entrance Length } \\
& R e=\text { Reynolds number } \\
& D q=\text { equivalent diameter }
\end{aligned}
$$

Considering the designated speed of $35 \mathrm{mph}(15 \mathrm{~m} / \mathrm{s})$, the BL reaches a maximum thickness of $0.45 \mathrm{~m}(1.5 \mathrm{ft})$ at the end of the wind tunnel, whilst the corresponding displacement thickness reaches a value of $0.05 \mathrm{~m}(0.16 \mathrm{ft})$.

Figure 3-8 shows that in order to compensate the boundary layer growth; a $5 \mathrm{~cm}$ divergence over $30 \mathrm{~m}$ length would be needed. For such a small value, it was decided to neglect the recommended divergence and proceed with a straight tunnel. This assumption was also justified in light of the fact that the plume might enter the boundary layer region only at the very end of the wind tunnel, where the plume evolution is expected to be already completed. 


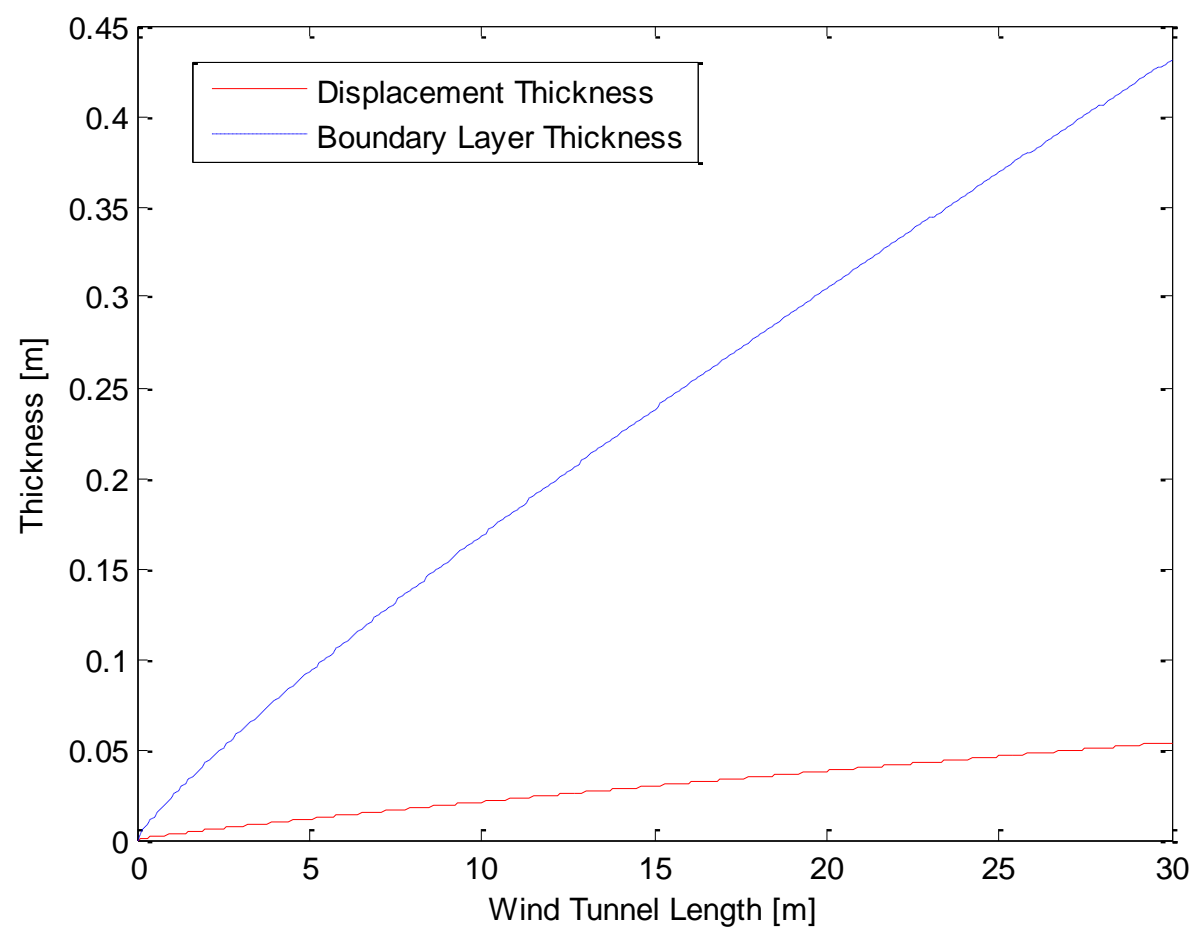

Figure 3-8: Boundary layer and displacement thickness growth within the WVU wind tunnel for a simulated wind speed of $35 \mathrm{mph}$.

The wind tunnel is designed for a wide range of applications and wind speeds and consequently, BL and DT were computed for a wide range of wind speeds. Figure 3-9 shows the results at a distance $30 \mathrm{~m}$ downstream the tunnel test cell entrance; it can be noted that the displacement thickness remains almost constant for the considered range of velocities and therefore, it may be concluded that its effect would play a small influence for any operating conditions within the design range. 
The walls of the wind tunnel are smoothly sanded, the joints between the plywood sheets are massaged, and a layer of epoxy is applied in order to guarantee a smooth finish and reduce as much of the growth of the BL as possible.

By analyzing Figure 3-8 and Figure 3-9, it can be concluded that the wind tunnel length is shorter than the entrance length needed to achieve fully developed conditions. It might be preferable for wind tunnel operations to always have a fully developed flow, a condition difficult to achieve when the size of the tunnel is in the order of $115 \mathrm{ft}$.

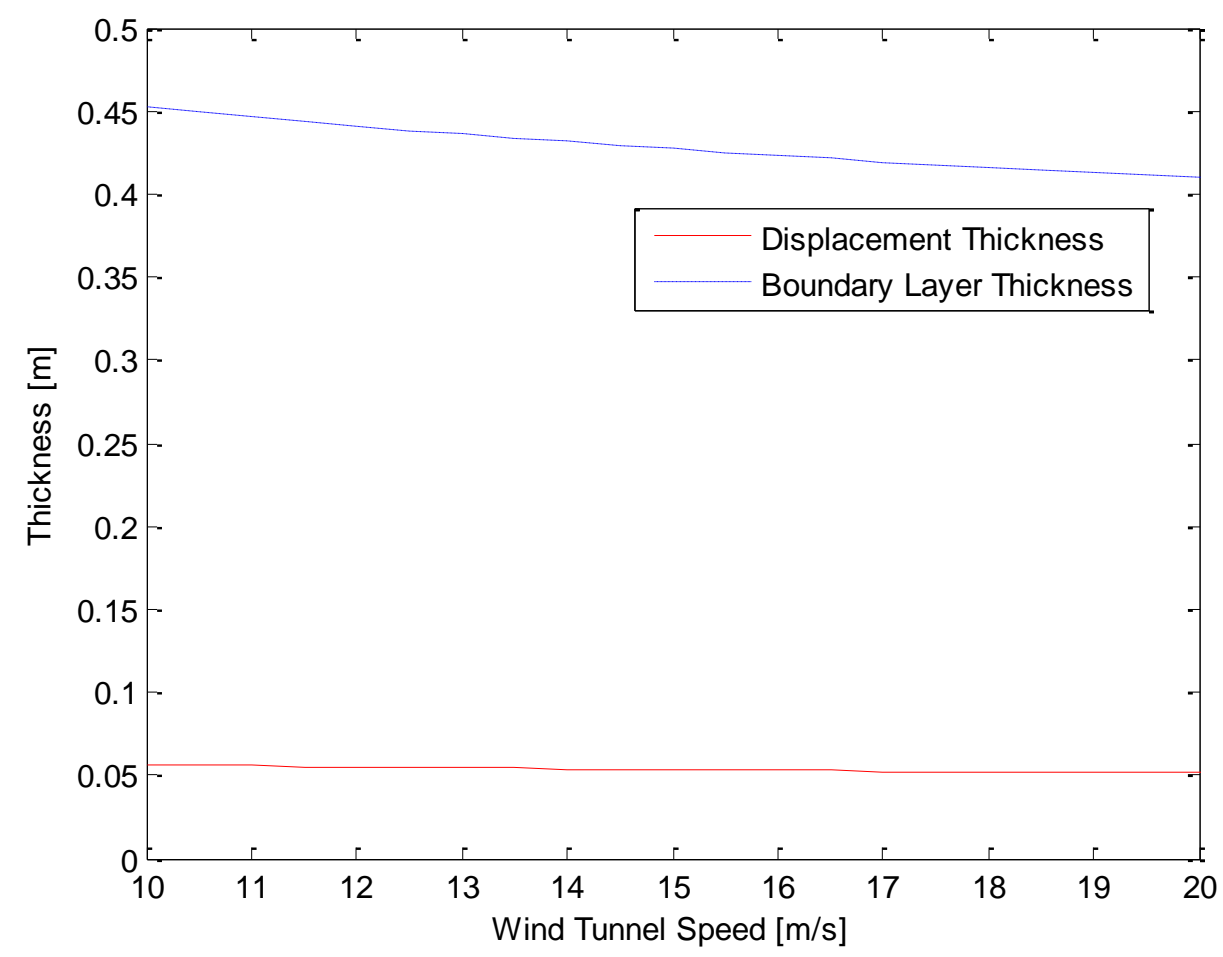

Figure 3-9: Boundary layer and displacement thickness for different simulated wind speeds at $30 \mathrm{~m}$ downstream the tunnel entrance. 
Rae et al. (1984) indicated that a wind tunnel exhibits a good quality flow if the velocity profile in the test cell is fully developed. The transition to fully developed flow should always be achieved prior the wind tunnel test cell. On the other hand, Rae et al. (1984) indicated also that a wind tunnel test cell with developing BL is more than acceptable, as long it does not interfere with the model object of study or the BL is in proximity to be fully developed.

\subsubsection{Finite Element Method Structural Analyses}

The needs for a specifically designed wind tunnel for plume investigation incurred several technical and structural problems. 


\subsubsection{Geometry Definition}

SolidWorks ${ }^{\mathrm{TM}}$ was used to define the final geometry of the wind tunnel. This geometry was exported to a finite element method (FEM) software. Femap ${ }^{\odot}$ with Nastran ${ }^{\odot}$ was used for the structural analysis. The geometry generated with SolidWorks ${ }^{\Theta}$ is observable in Figure 3-10 and Figure 3-11.

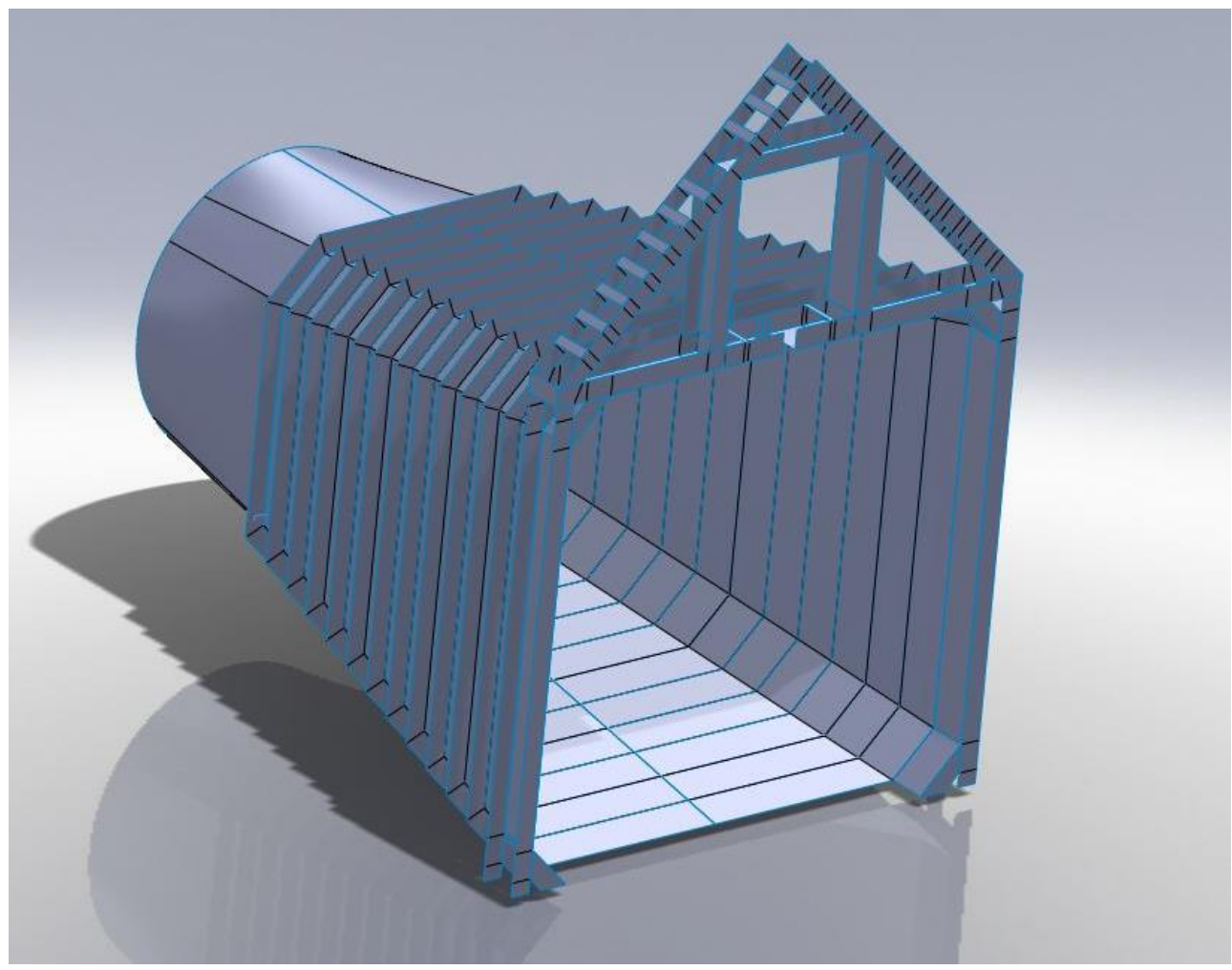

Figure 3-10: Wind tunnel CAD model for structural analyses during SolidWorks ${ }^{\odot}$ generation, $3 \mathrm{D}$ view 


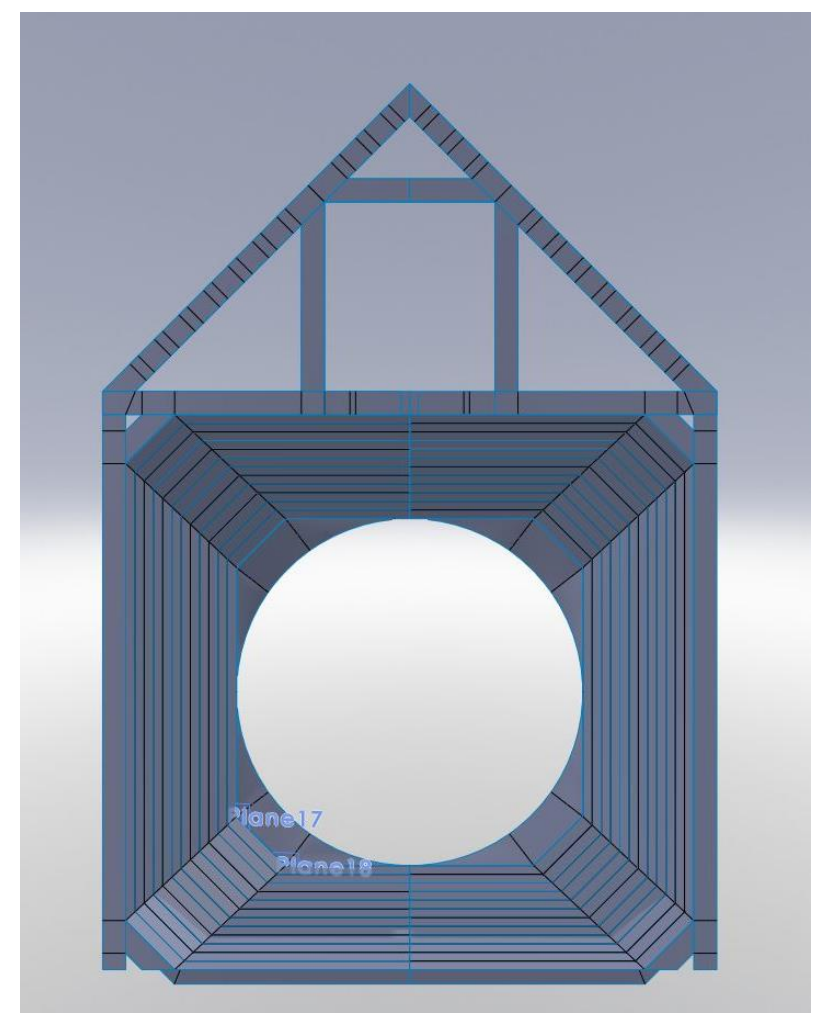

Figure 3-11. Wind tunnel CAD model for structural analyses during Solidworks ${ }^{\circledR}$ generation, front view

Figure 3-12, shown below, depicts the final part of the wind tunnel, where the propeller is placed. Figure 3-13 shows the corridor built for the cart, including guidance rails. The roof has a double role, to act as both a regular roof, as well as a structural truss member to accommodate the slot for the cart. A standard 36in size door and a screen, shown in Figure 3-14, were modeled in order to better understand the concentration of stresses generated by their presence. 


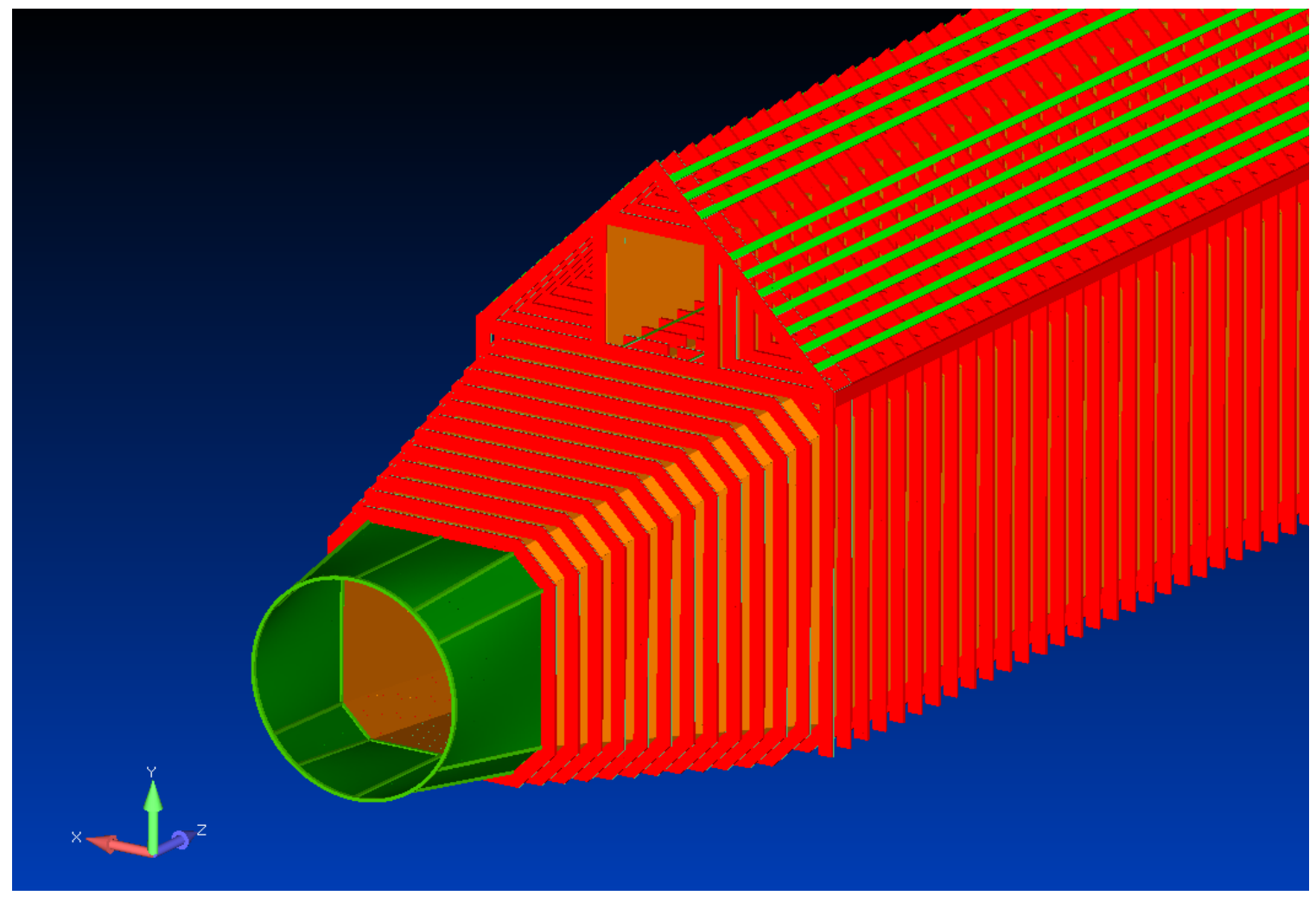

Figure 3-12: Wind tunnel CAD model for structural analyses after being imported in Femap(C, 3D view shows the propeller section 


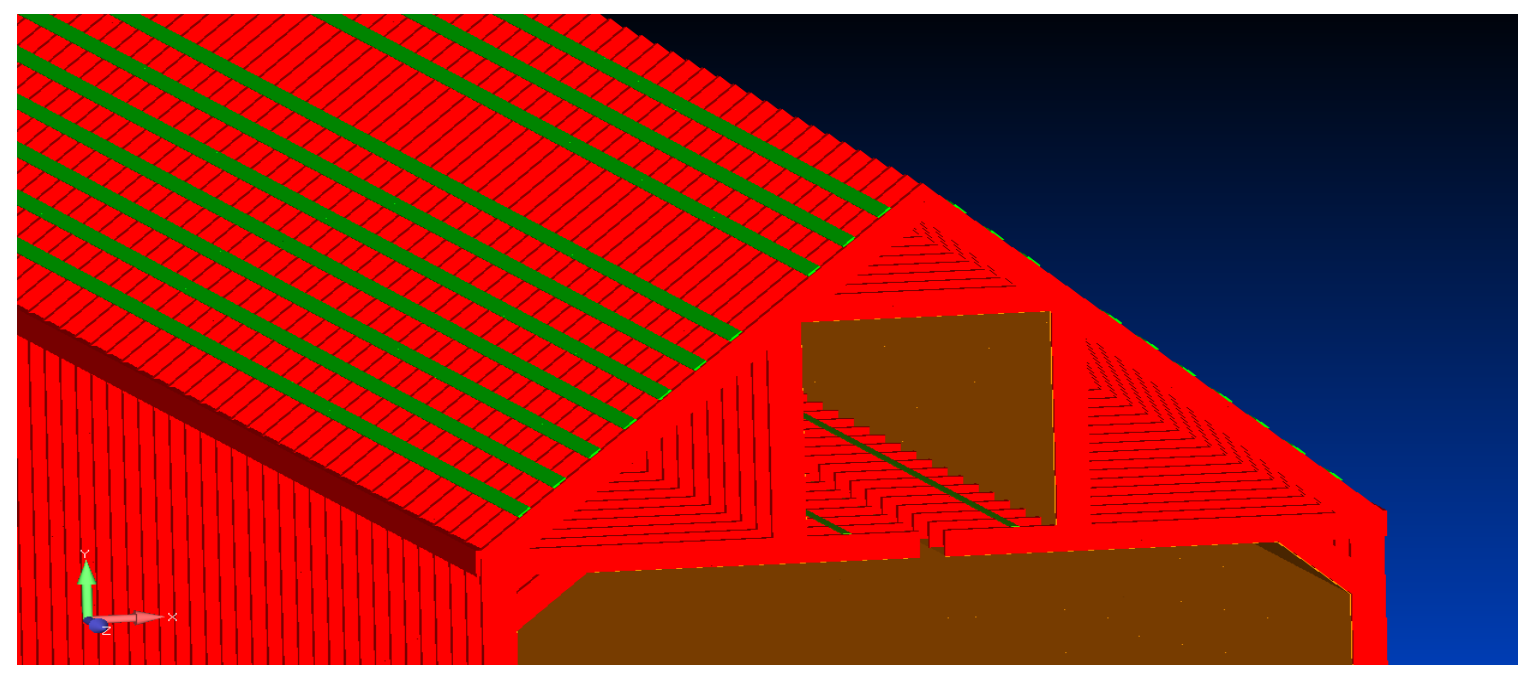

Figure 3-13: Wind tunnel CAD model for structural analyses after being imported in Femap $($, 3D view shows the cart corridor and the slot in the ceiling

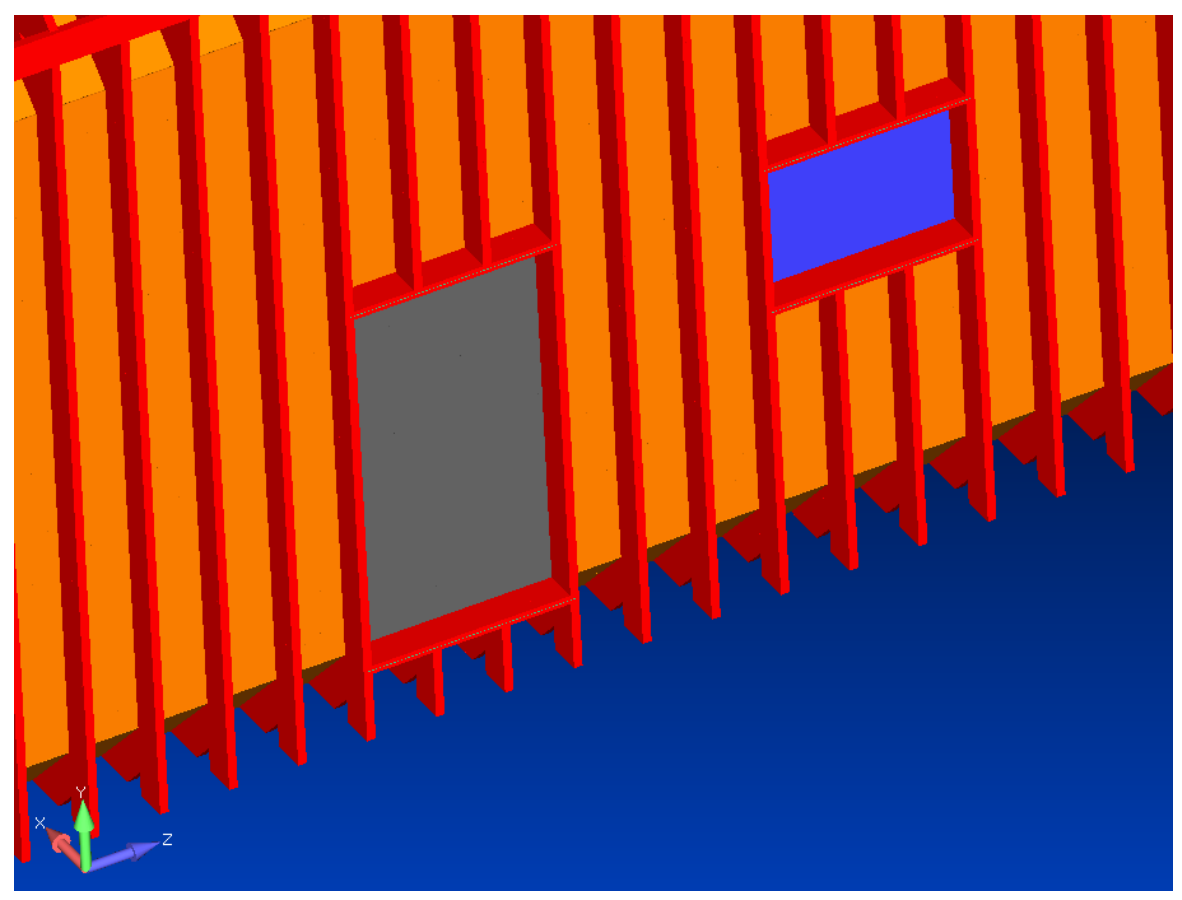

Figure 3-14: Wind tunnel CAD model for structural analyses after being imported in Femap $($, , 3D view shows the access door and the screen 


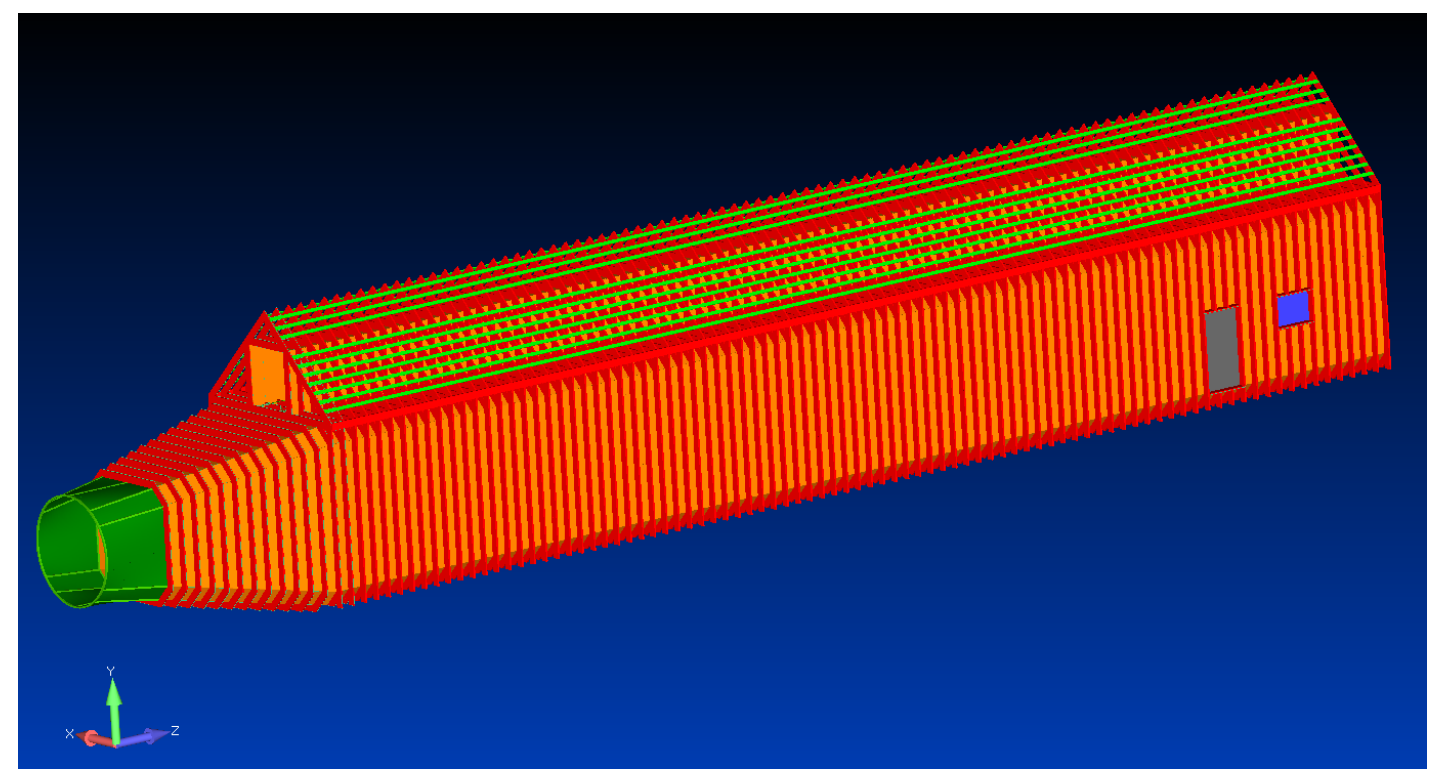

Figure 3-15: Wind tunnel CAD model for structural analyses after being imported in Femap(C, 3D view shows the entire geometry

Please note the entrance for truck or maintenance vehicles, is realized by removing the flow straightener section in front of the wind tunnel, in order to not weaken the structure and have the largest opening possible. In this structural analysis, the flow straightener has not been considered, because it does not play a structural role in the overall wind tunnel.

\subsubsection{Material Properties}

The first step for the structural analysis is to define the materials of the structure. In this case, structural steel (Figure 3-16) is used for metal parts, structural pine wood (Figure 3-17) for the entire wind tunnel, and acrylic glass $\left(\right.$ Plexiglas $^{\circledR}$ ) for the screen. The differences between Figure 3-16 and Figure 3-17 can be seen in that the steel is considered 
isotropic material, thus its properties do not change as a function of the direction of the load, while the pine wood is orthotropic. An orthotropic material presents two different directions of properties, this is related to the intrinsic composition of fiber and resin of the wood itself, and therefore it results stronger with the load along the fibers, while weakening if loaded across the fibers.

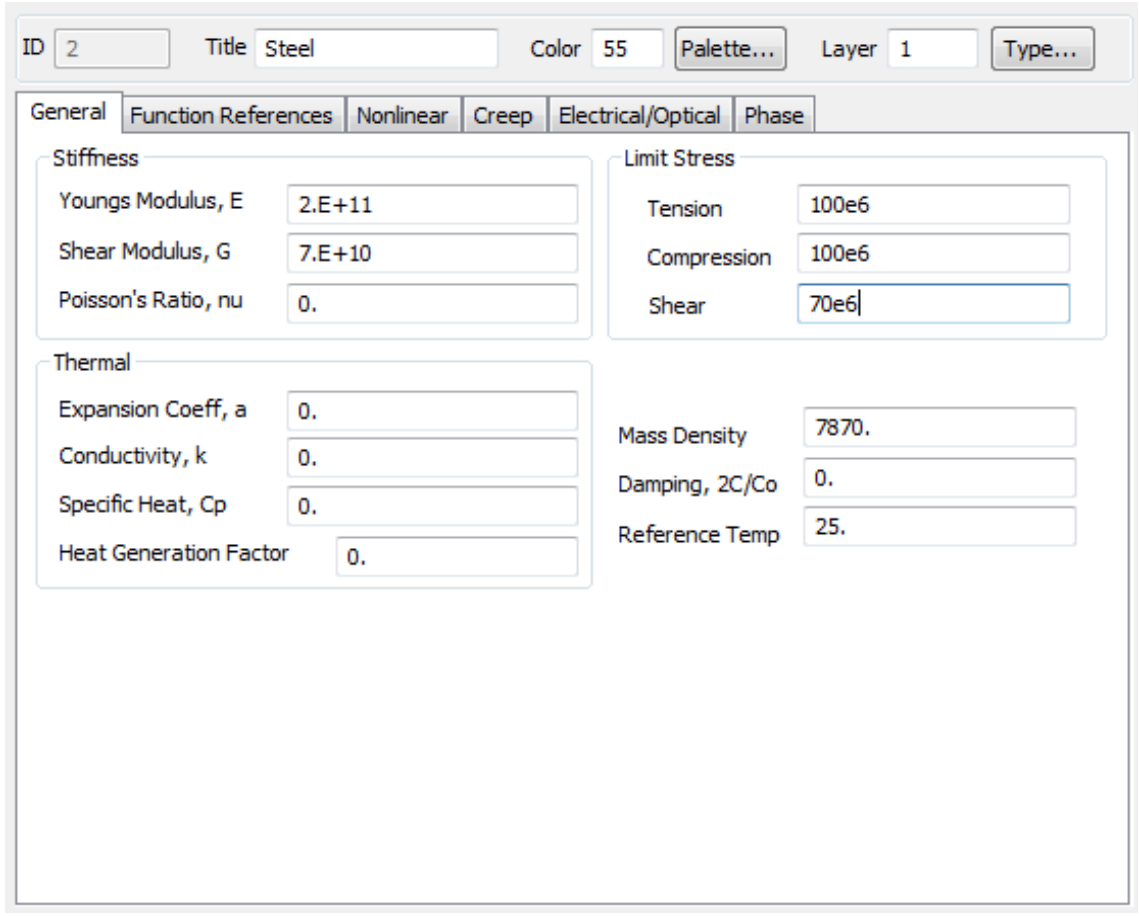

Figure 3-16: Femap $($ screenshot indicating the material properties for the structural steel 


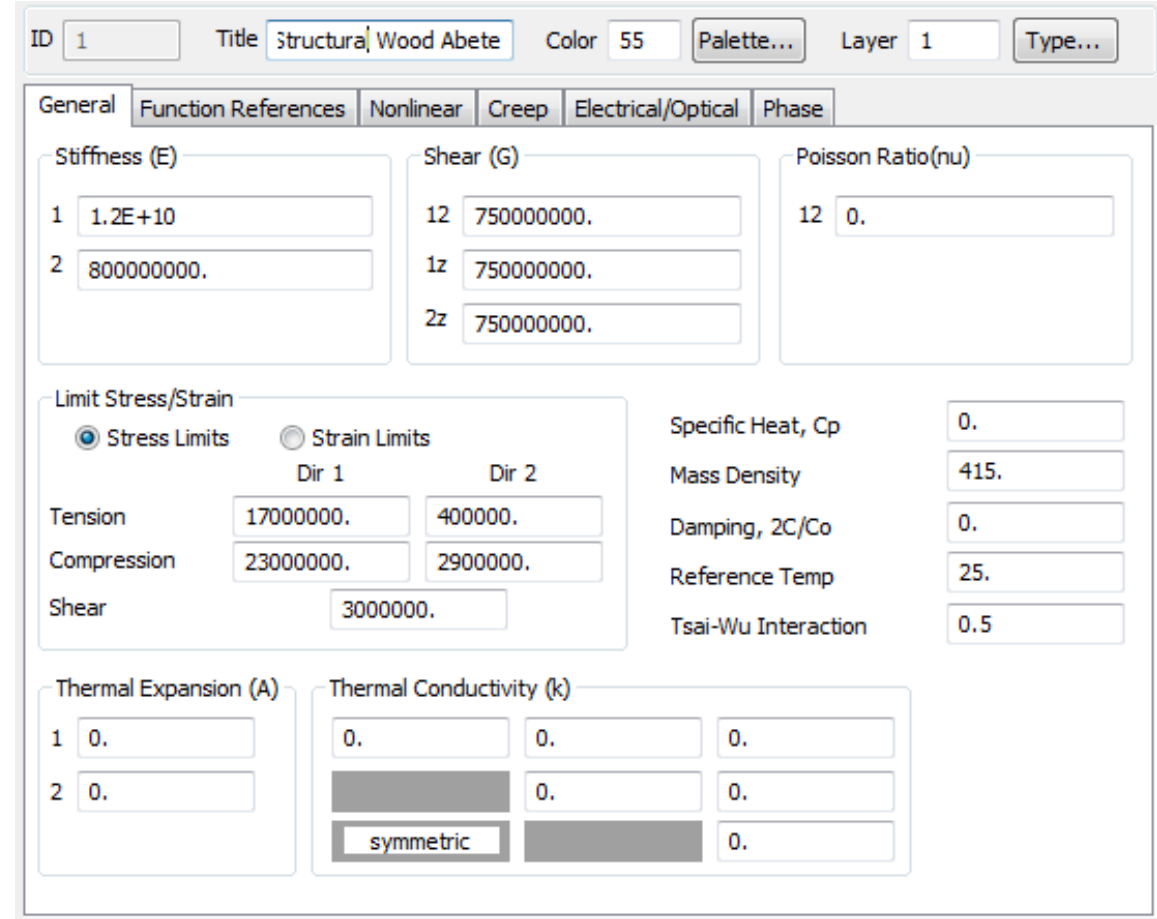

Figure 3-17: Femap $\odot$ screenshot indicating the material properties for the structural pine wood

\subsubsection{Element Properties}

A critical modeling component is the definition of the element properties. As shown in the previous images, surface elements have been chosen considering improved mesh quality, computational time, and type of problem. Each element has been defined as a laminate element, whereby the Failure Index Method (FIM) (with Tsai-Wu interaction theory) is more adequate, as opposed to the von Mises Criterion due to the presence of wood (an orthotropic material). Although the structure was defined using laminate elements, the substitution of a steel plate element (isotropic material) yields qualitative information. In addition, this provides for a reduction in computational time, and the 
quantitative information allows one to assign a Failure Index for stresses modeled for the structure. Due to the nature of the Failure Index, it provides for a PASS or NON-PASS testing procedure. The structure is considered safe from failure if the Failure Index remains within the range of 0 and 1 . Figure 3-18 illustrates a general laminate element.

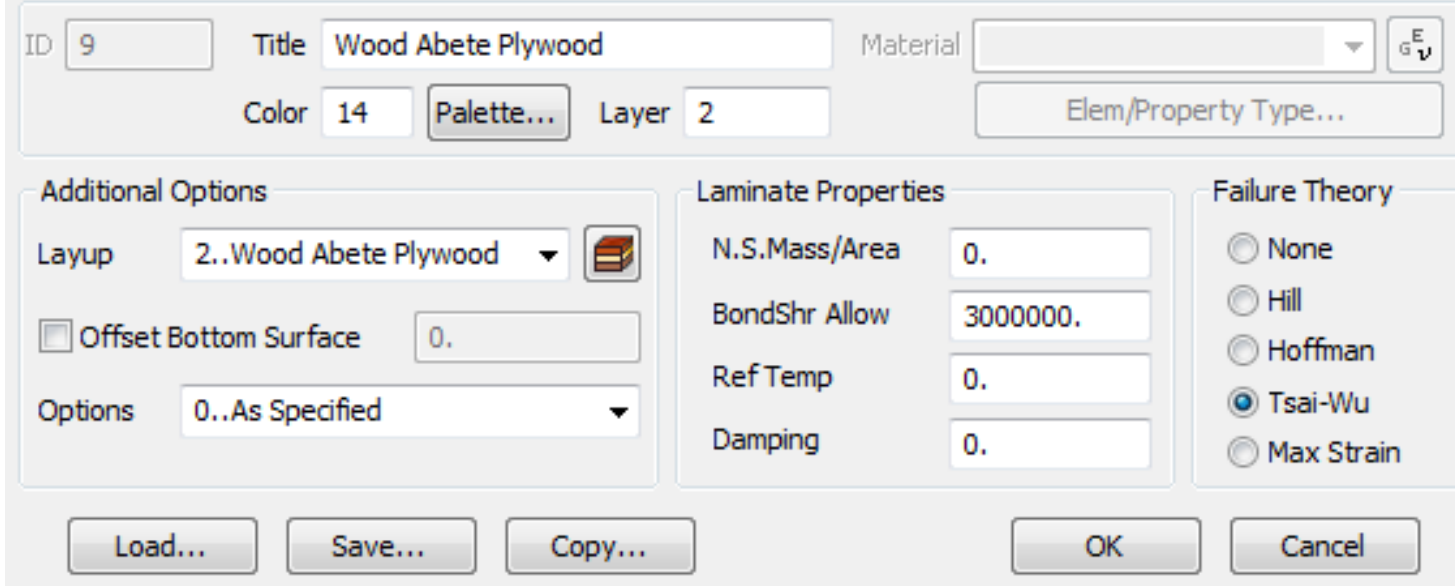

Figure 3-18: Femap( screenshot of the general structural laminate element properties

\subsubsection{Mesh Definition}

The mesh generated with described parameters presents a total number of 282,125 of laminate elements, as illustrated in Figure 3-19. Due to the symmetry of the problem and the regular shape of the structural elements (such as the vertical studs and plywood plate), the mesh results are geometrically simple and well structured, leading to an overall high mesh quality. 


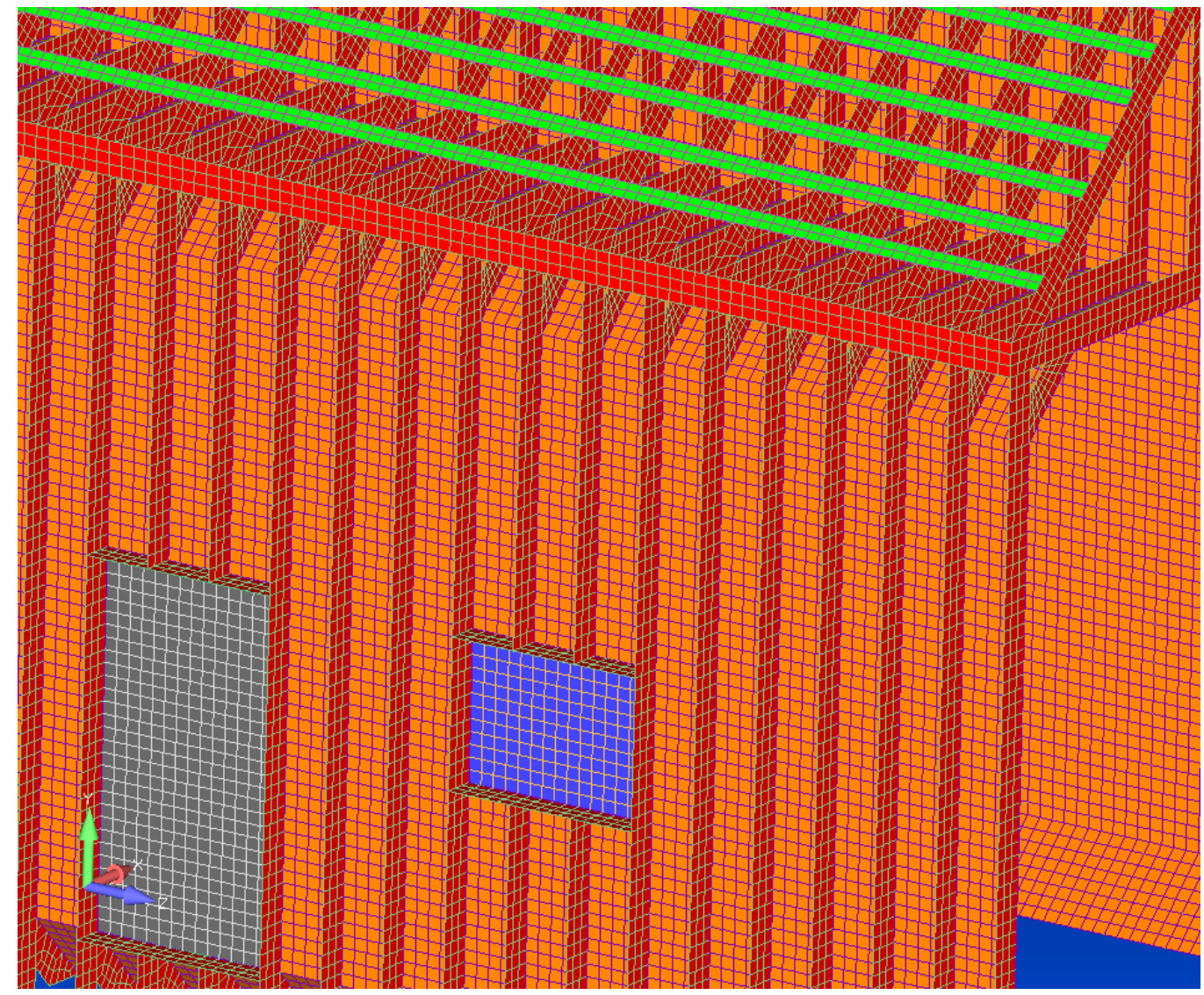

Figure 3-19: Femap $($ screenshot illustrating the mesh for the structural analyses, it is a visible well-structured mesh

\subsubsection{Load Sets and Constraint Sets}

This project represents a combination of different analyses due to the complexity and dimensions of the structure itself. 
The first load set is related to the operational condition of the wind tunnel:

- 2,000 Pa differential pressure over the internal plate of the wind tunnel for the constant section part.

- 2,500 Pa differential pressure over the internal plate of the wind tunnel for the convergent part (the increase in velocity reflects into and increment of differential pressure).

- $\operatorname{Gravity}\left(9.81 \mathrm{~m} / \mathrm{s}^{2}\right)$.

- $500 \mathrm{~kg}$ cart placed in each elemental section of the wind tunnel.

- $1,000 \mathrm{~N}$ force placed on the first portal, along the longitudinal axis, to replicate the load of the flow straightener and screens.

These loads are defined, thanks to previous CFD analysis, considering maximum rounded observed values. In addition, they were doubled for safety reasons.

The second load set is related to the weather conditions of the Morgantown area. A snow accumulation of $1 \mathrm{~m}$ over the entire roof with the heaviest snow possible $\left(500 \mathrm{~kg} / \mathrm{m}^{3}\right)$ was considered. This results in a snow loading during an extraordinary winter. This condition is the worst possible scenario, resulting in a 5,000 $\mathrm{Pa}$ overloading of the entire roof. Moreover, considering a $45^{\circ}$ roof pitch, such accumulation would not likely be realized.

The final load set is identified by a $50 \mathrm{mph}$ wind on the lateral surface of the wind tunnel. The value of the load is calculated considering the entire lateral surface as a 
stagnation region for the wind. This hypothesis will be more severe than any real conditions. Such a wind load results in a $300 \mathrm{~Pa}$ over the entire exposed lateral surface (roof included).

The values for wind speed and snow load were chosen analyzing the historic data for the Reedsville (WV) area of the last 75years available on the National Oceanographic and Atmospheric Administration website (www.NOAA.com) and International Building Code (IBC Counsil, 2009). The base of the entire structure is fixed to the ground.

\subsubsection{Structural Results}

This simulation corresponds to the load defined for the operational conditions. It is possible to observe in the following images (Figure 3-20 to Figure 3-23) that the structure completely satisfies the threshold identified by the FIM. The presence of the door and the screen does not affect the integrity of the structure. The Failure Index Contour is between 0 and 0.7 . 


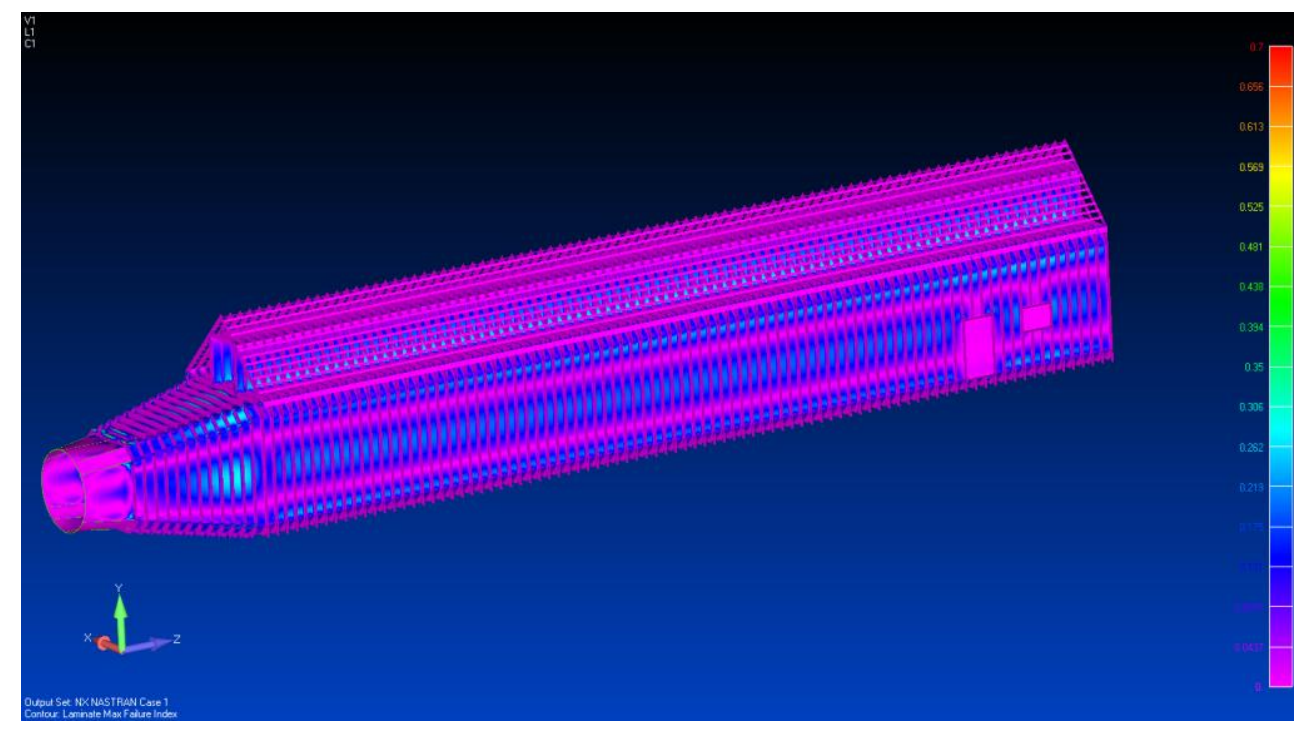

Figure 3-20: Operational conditions structural analysis results, overview of the entire model, maximum value observed is 0.3

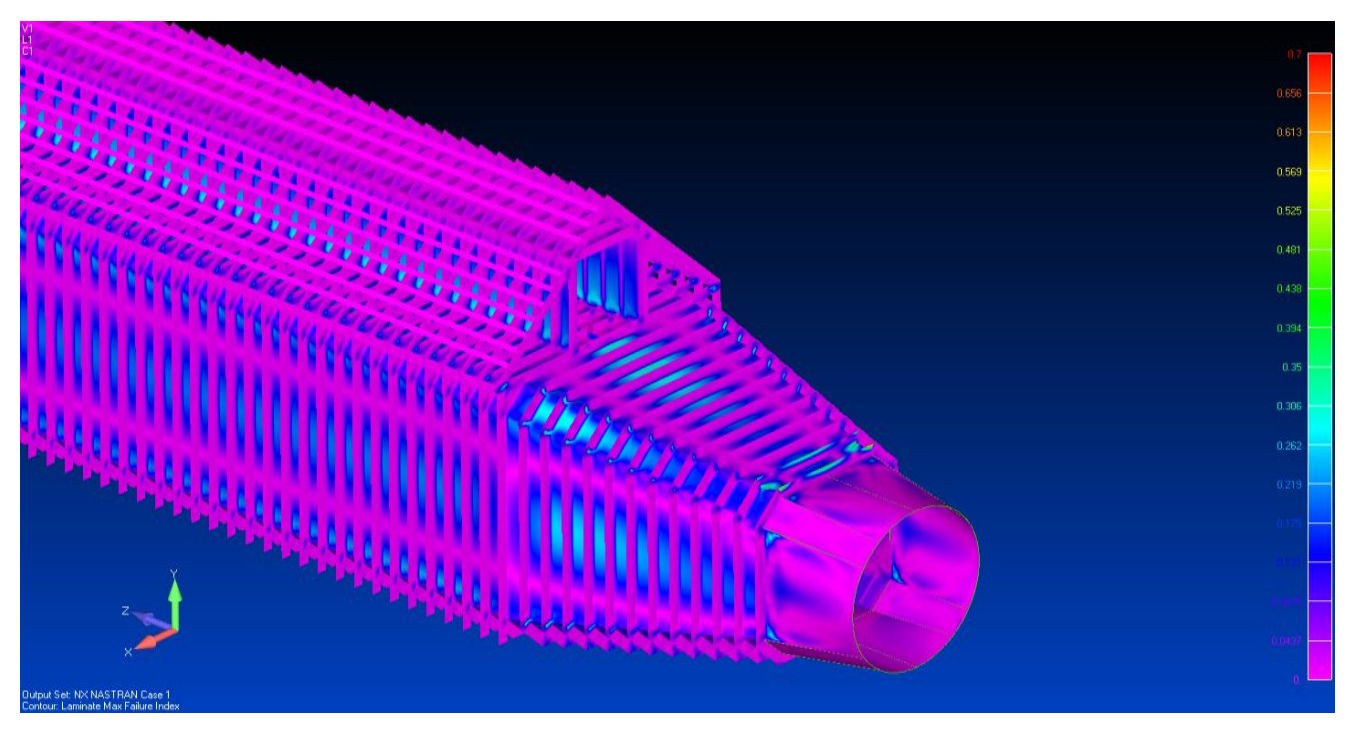

Figure 3-21: Operational conditions structural analysis results, detail of the convergent propeller section, maximum value observed is 0.3 


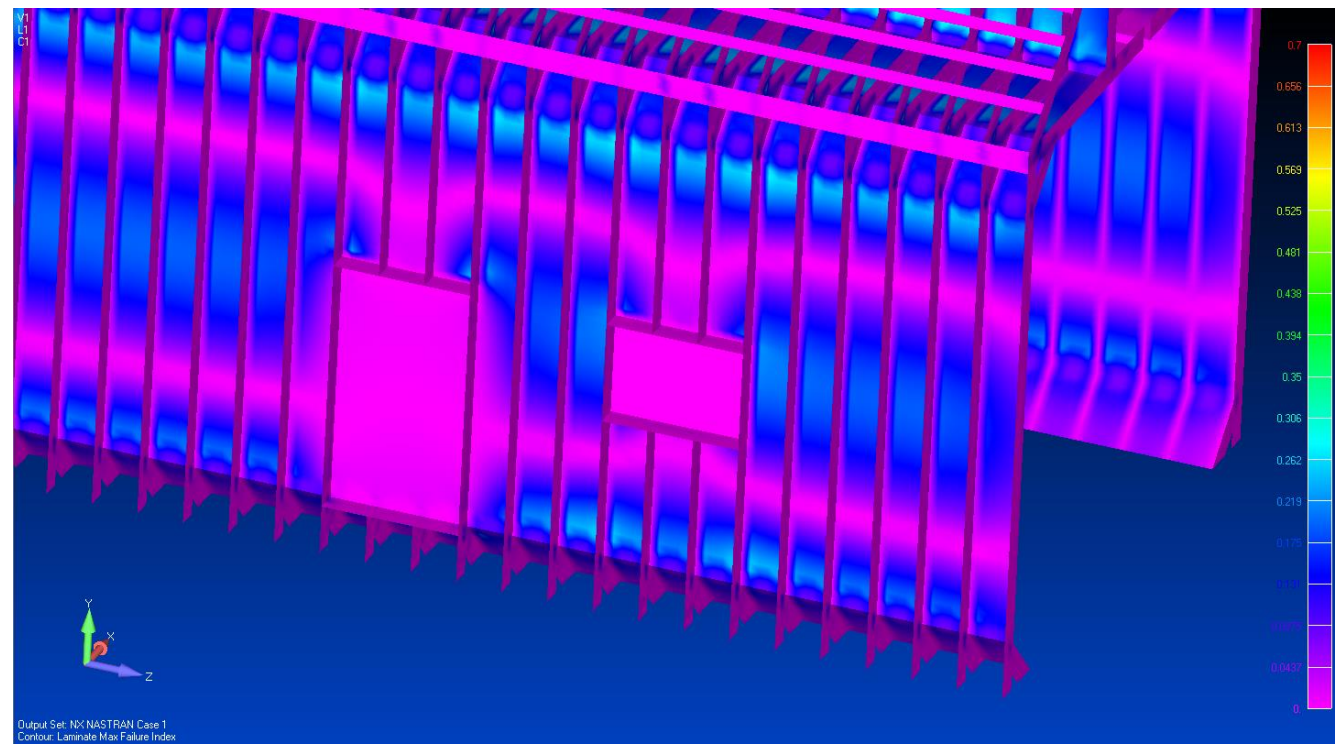

Figure 3-22: Operational conditions structural analysis results, detail of door and screen, maximum value observed is 0.3

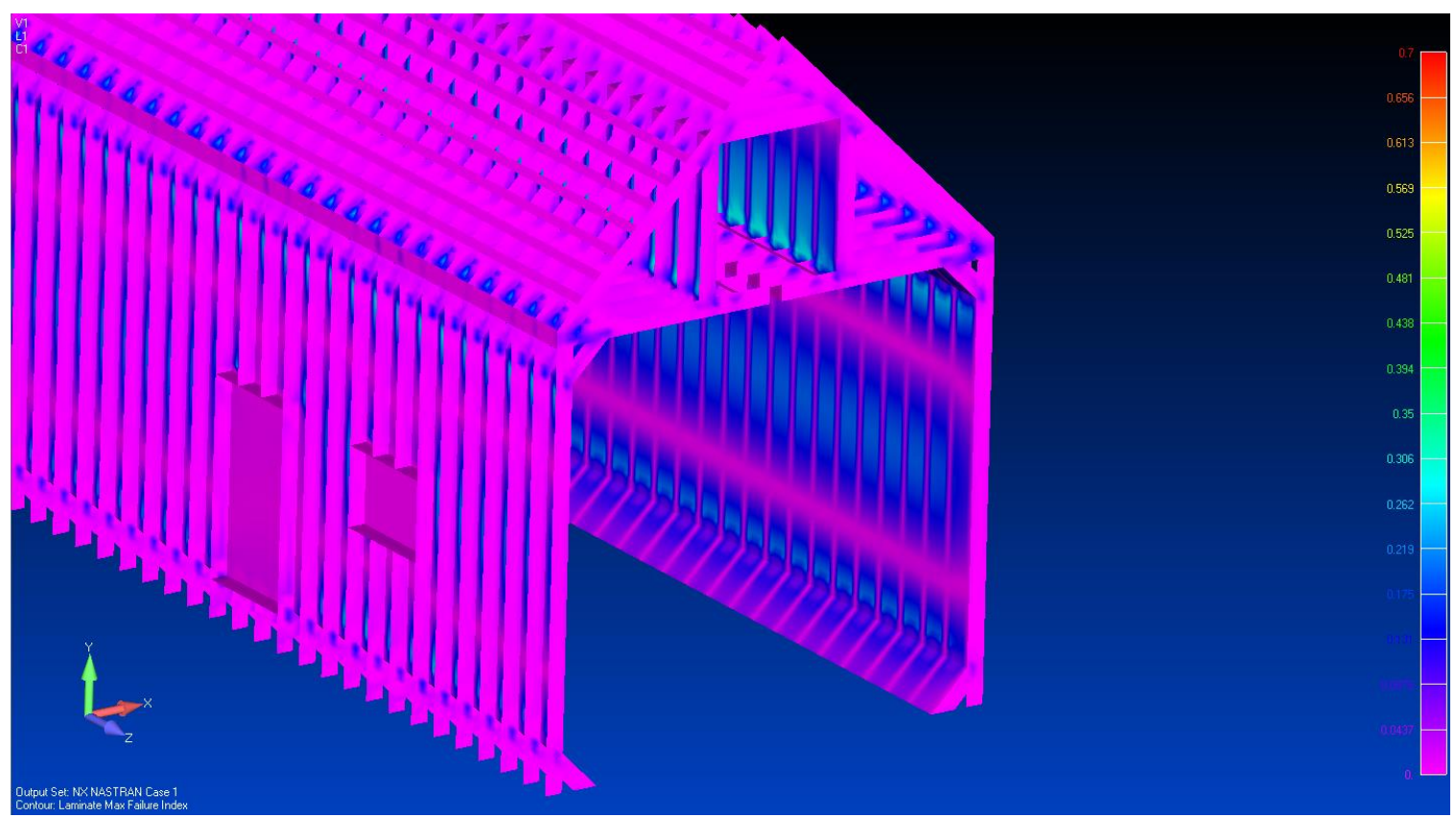

Figure 3-23: Operational conditions structural analysis results, detail of the entrance section and instrument cart corridor, maximum value observed is 0.25 
Applying the load set described previously for the Snow Accumulation Condition, Figure 3-24 and Figure 3-25 show that it is clear that the structure can withstand these conditions with a considerable margin for safety and performance. Applying the load set described previously for lateral wind conditions, shows it is clear that the structure can withstand these conditions with a substantial safety level as well.

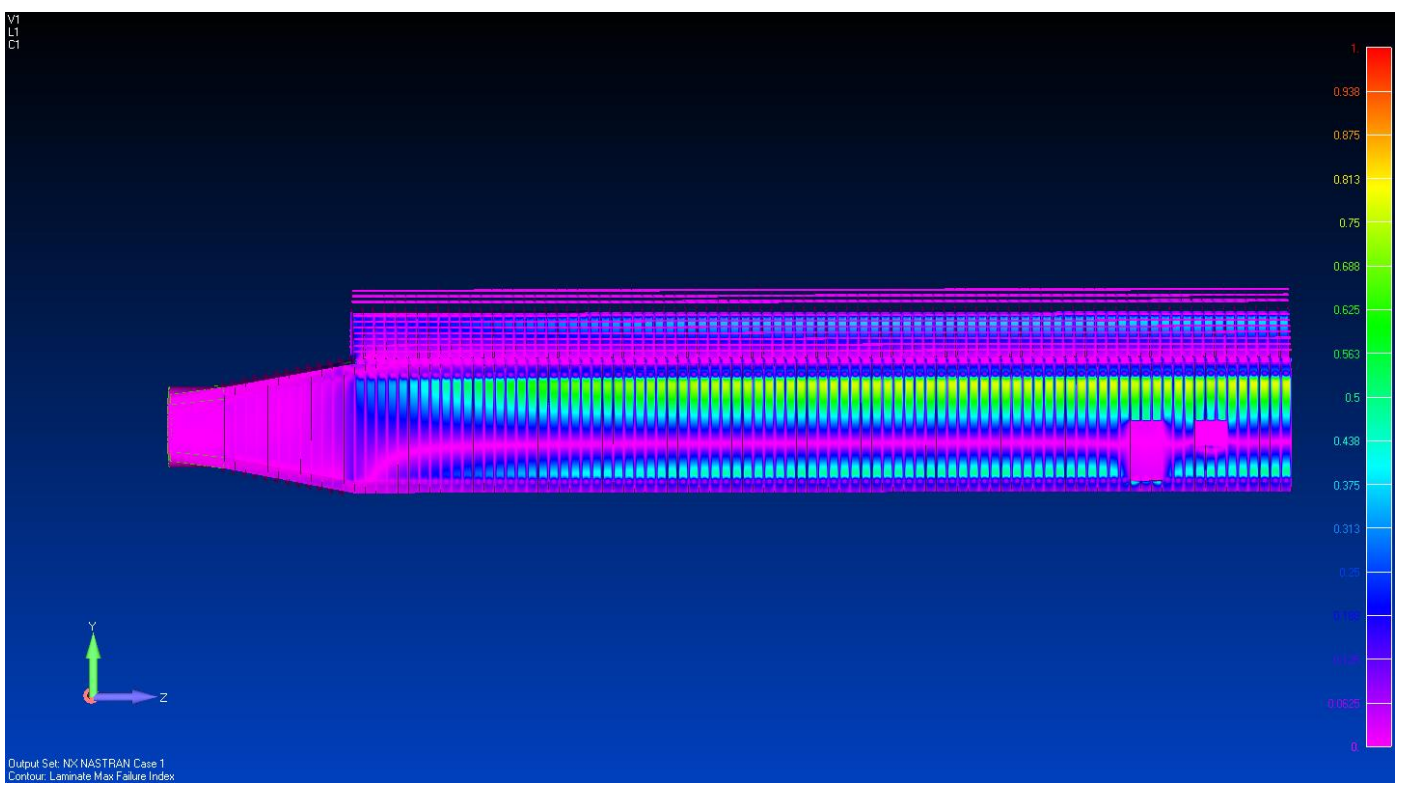

Figure 3-24: Snow accumulation conditions structural analysis results, overview of the entire model, maximum value observed is 0.8 


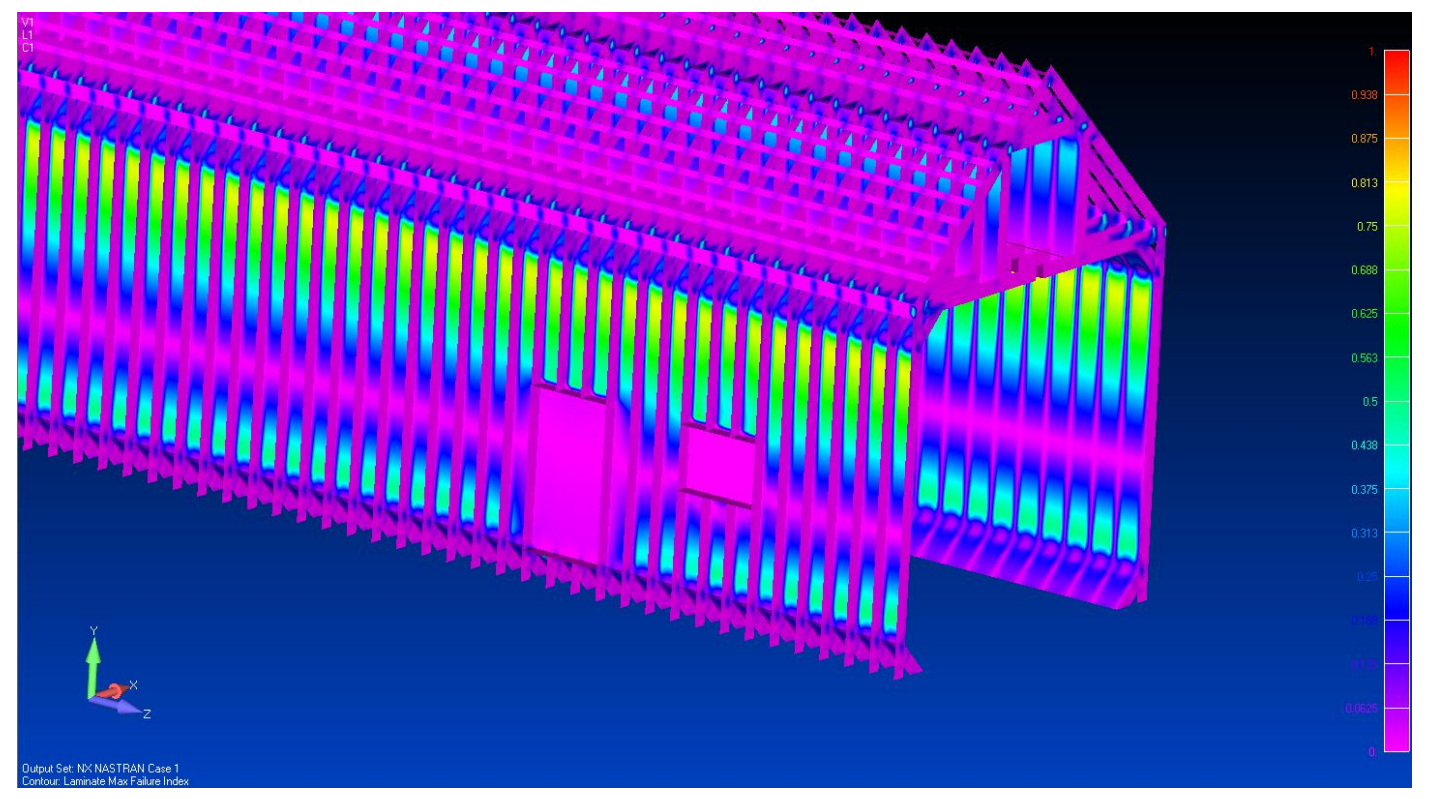

Figure 3-25: Snow accumulation conditions structural analysis results, detail of the entrance section, instrument cart corridor and entrance door with screen, maximum value observed is 0.8

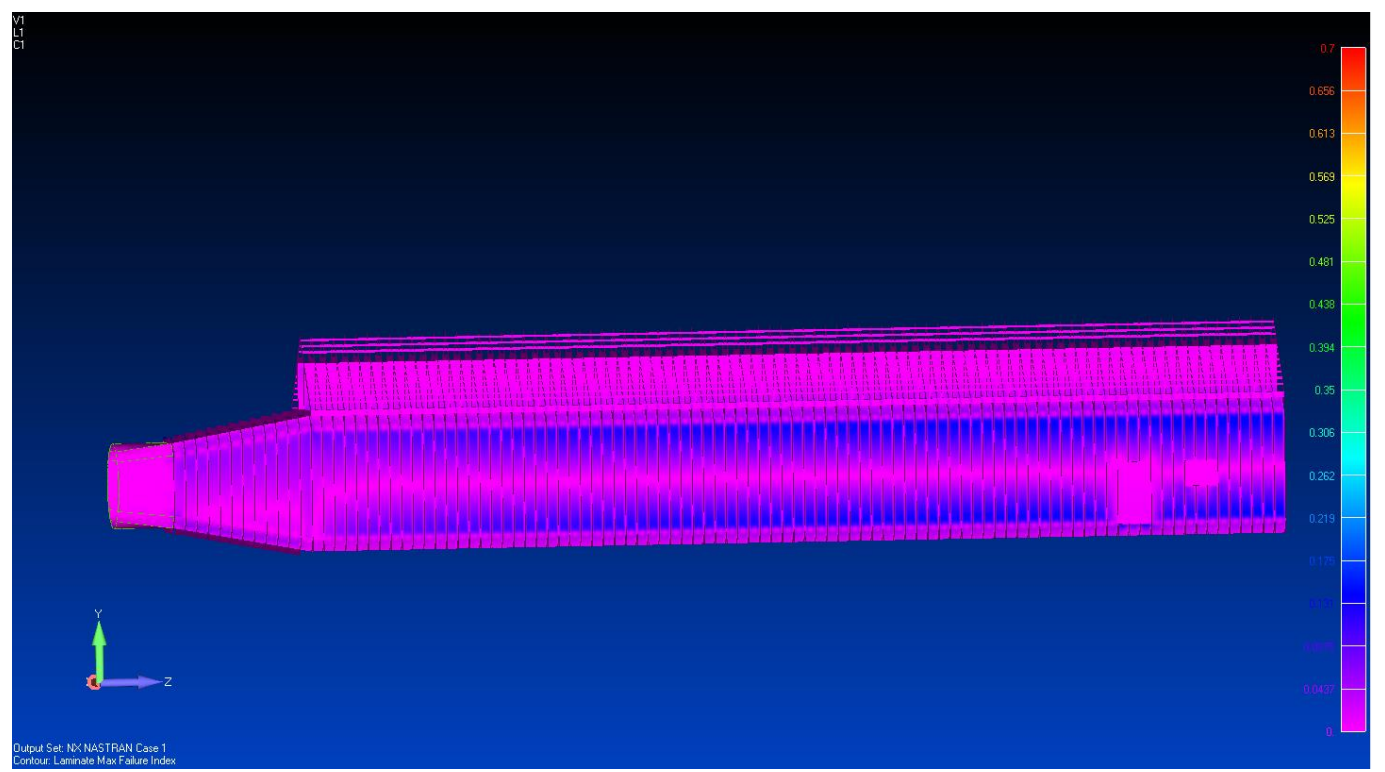

Figure 3-26: Lateral wind conditions structural analysis results, overview of the entire model, maximum value observed is 0.25 


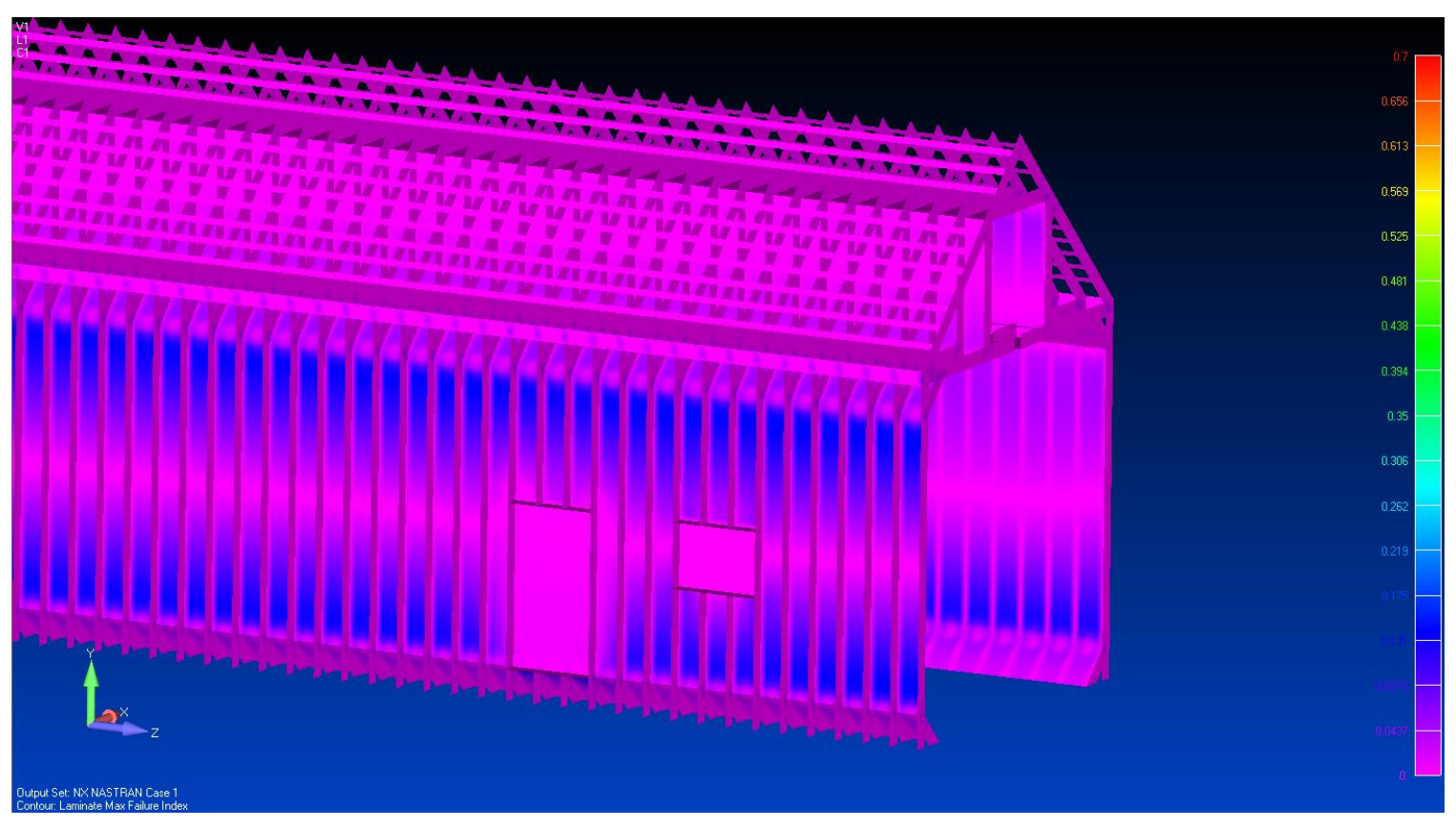

Figure 3-27: Lateral wind conditions structural analysis results; detail of the entrance section, instrument cart corridor, and entrance door with screen

Due to the operational conditions of the wind tunnel, it is extremely important to verify that no resonance problems occur. The excitation frequency generated by a fiveblade propeller that is being powered by an engine operating between 1000 and $2000 \mathrm{rpm}$ is in the range of $85-165 \mathrm{~Hz}$. Femap@ is able to indicate the first ten natural frequencies of the model. It is important to remember that only the first five values shown have a physical relevance. The results are collected and displayed in

Table 3-1.

Table 3-1: Wind tunnel natural frequencies analysis and comparison of the excitation frequency during operational conditions 


\begin{tabular}{lc}
\hline Frequency & Parameter \\
\hline Excitation Frequency (5 blades) & $85-165$ \\
\hline $\mathbf{1}^{\text {st }}$ Natural Frequency & 4.45 \\
$\mathbf{2}^{\text {nd }}$ Natural Frequency & 5.88 \\
$\mathbf{3}^{\text {rd }}$ Natural Frequency & 9.06 \\
$\mathbf{4}^{\text {th }}$ Natural Frequency & 11.81 \\
$\mathbf{5}^{\text {th }}$ Natural Frequency & 13.13 \\
\hline
\end{tabular}

It is clear that no problem of resonance will occur, since the excitation frequencies are higher than the natural frequencies by a one order of magnitude, guaranteeing a safe margin from resonance problem. Figure 3-28 illustrates the deformed shape of the model for the first natural frequency with an amplification factor for the deformation of 30 . 


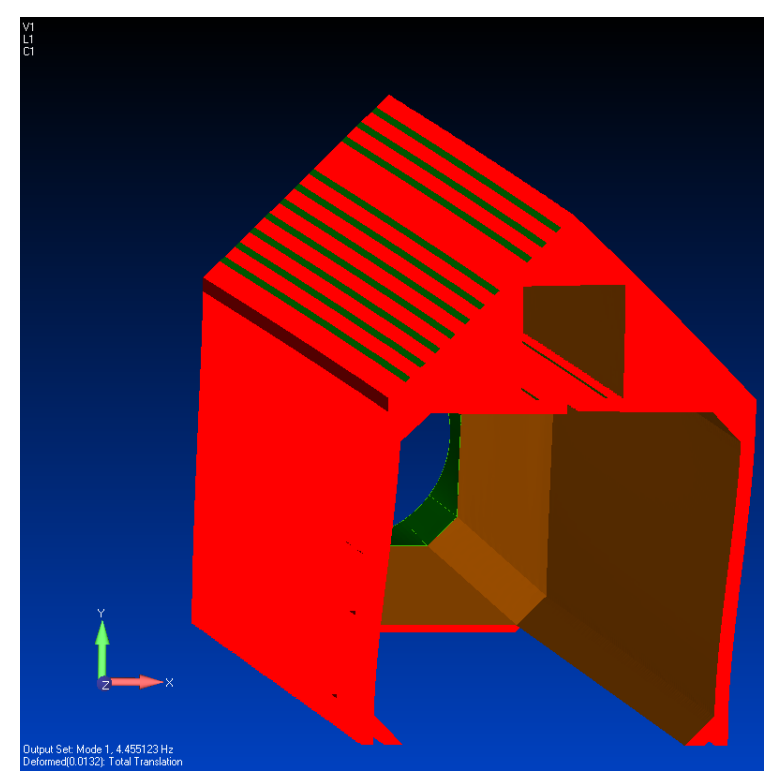

Figure 3-28: First natural frequency for the wind tunnel, the deformation is showed with an amplification factor of 30

Due to the vacuum generated in the wind tunnel and the related compression loading on the structure, it is important to check that no buckling problems of the structure itself could occur during the operational conditions. The result obtained by Femap ${ }^{\circledR}$ shown in Figure 3-29, does not reflect the symmetry of the problem. These are only mathematical results (eigenvalue of the load is 8 ), without physical values. Thus, it is possible to say that buckling will not occur, given the design inputs and boundary conditions. 


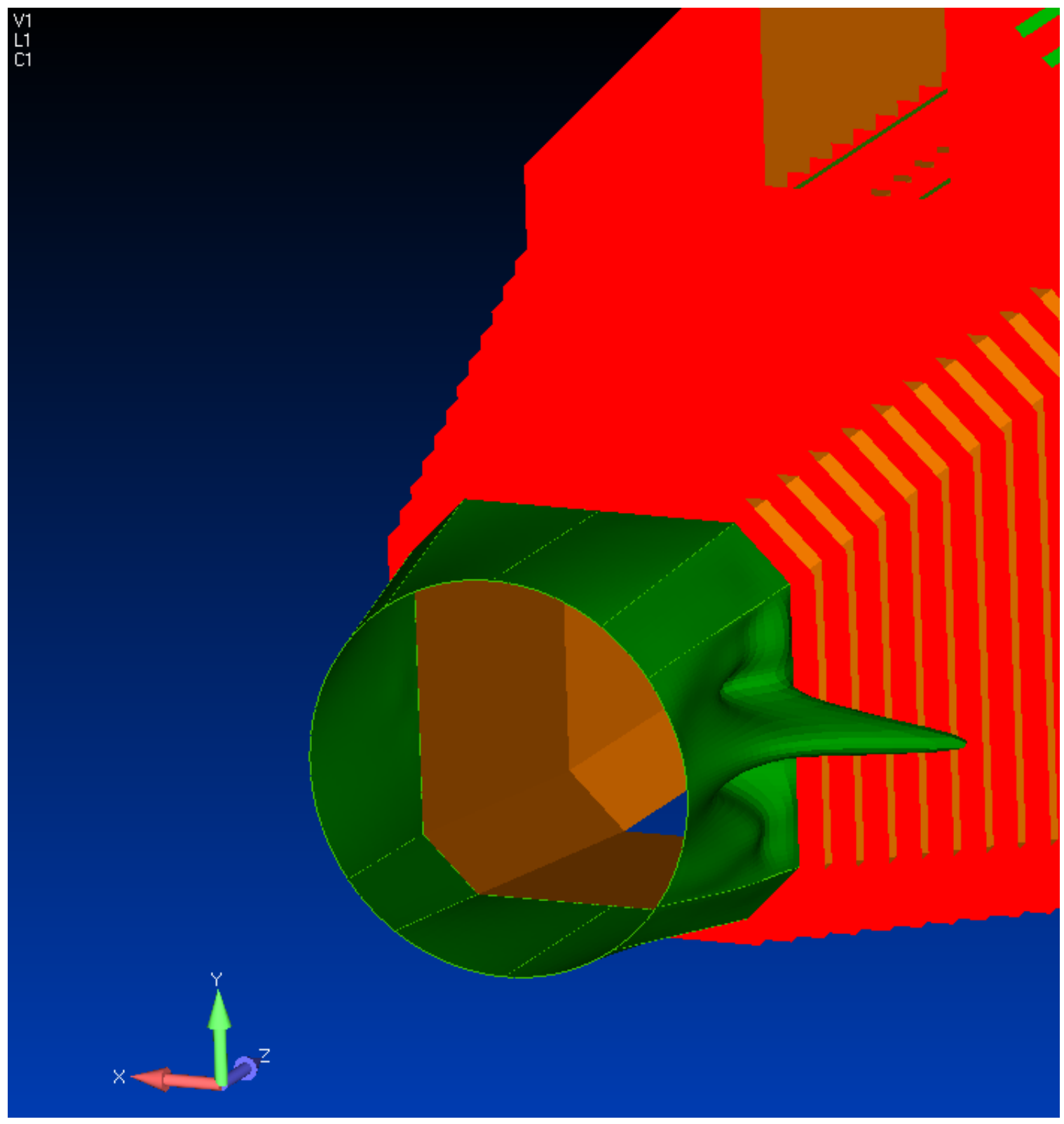

Figure 3-29: Wind tunnel buckling results, details of the convergent propeller 


\subsection{Wind Tunnel Construction}

The construction of the wind tunnel is divided into five major subtasks:

- Main Structure

- Engine and Propeller

- Services (Electric and Compressed Air system)

- Data Acquisition System (DAQ) and Instrumentation

- Movable Platform

The structure of the wind tunnel is composed of three main components. The concrete floor and footers provide the foundation for the wood structure, which is the second component. The remaining component is the metal convergent structure in front of the wind tunnel that will stabilize the internal flow within the wind tunnel, minimizing turbulence intensity, in order to provide for a repeatable, quantifiable flow field. The structural components were chosen upon the FEA results observed in the structural analysis.

Table 3-2 details the components of the wood structure while Table 3-3 shows the components of the metal structure. Several solutions have been put into place to guarantee the safe construction results. The side-walls of the wind tunnel are composed at the bottom of a seven inch concrete curb, in order to prevent the direct contact of the wood with stagnant water or snow. Metal siding was fitted into the terrain to protect even more from rain or outside snow accumulation. In addition, a French drain and gravel have been incorporated to allow for expedited draining, should precipitation occur. 
Table 3-2: Wind Tunnel Wood Main Structural Components

\begin{tabular}{lc}
\hline Component & Qualification \\
\hline 2'x8'x16' Kiln Dried Lumber & Section Component \\
\hline 2'x8'x16' Treated Lumber (grade 1) & Base Component \\
\hline '"x4'x16' Kiln Dried Lumber & Roofing Fixing \\
\hline 4'x8'x1/2" Plywood BC & Internal Covering \\
\hline 4'x8'x1/2" Plywood CC & Gusseting \\
\hline 4'x8'x3/4" Plywood CC & Jig Section \\
\hline Metal Roofing & Galvanized Steel \\
\hline Metal Siding & Galvanized Steel \\
\hline
\end{tabular}

Table 3-3: Wind Tunnel Metal Main Structural Components

\begin{tabular}{lc}
\hline Component & Qualification \\
\hline 2"x2"x0.125" Steel Square Tube & Section Component \\
\hline 2mm Steel Plate & Internal Covering \\
Metal Roofing & Galvanized Steel \\
Metal Wall & Galvanized Steel \\
\hline
\end{tabular}


On top of the curb and over its entire length, a pressure treated piece of lumber has been connected with Tapcon ${ }^{\circledR}$ screws and hurricane straps. The studs of the side-wall are then connected to pressure-treated lumber with screws. At every connecting joint of two pieces of pressure-treated lumber, a king stud has been fastened into place (see Figure 3-30). All the joints of the truss and sidewall have been reinforced with a $1 / 2$ in. gusset (as shown in

Figure 3-31). Two emergency doors were constructed, one at the center and one at end of the wind tunnel test section. In order to reinforce the door frame, a 2" $\mathrm{x} 8$ " header has been built with king studs, as shown in Figure 3-30.

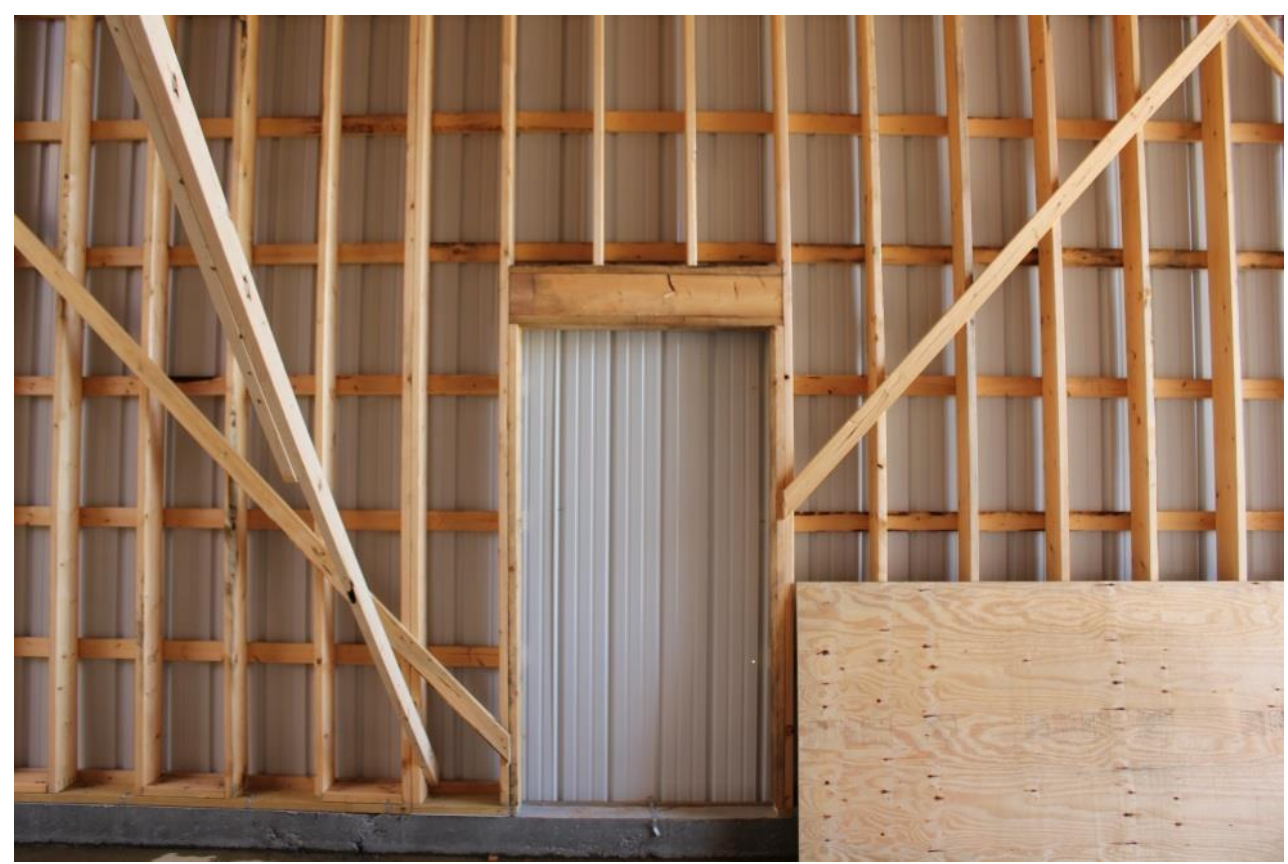

Figure 3-30: View of the internal wall of the wind tunnel, details of the door opening and king stud reinforcement 


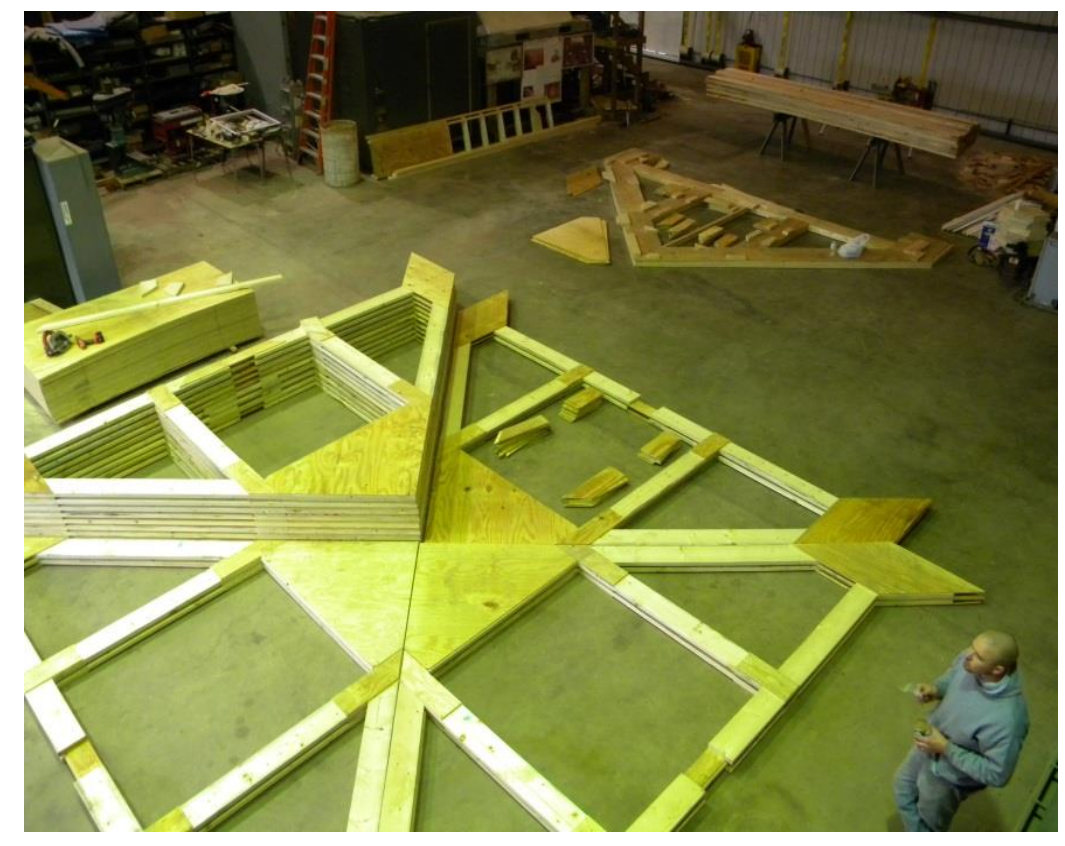

Figure 3-31: Fabrication of the wind tunnel wood trusses, details of the 1/2" plywood gusset reinforcement of the joints

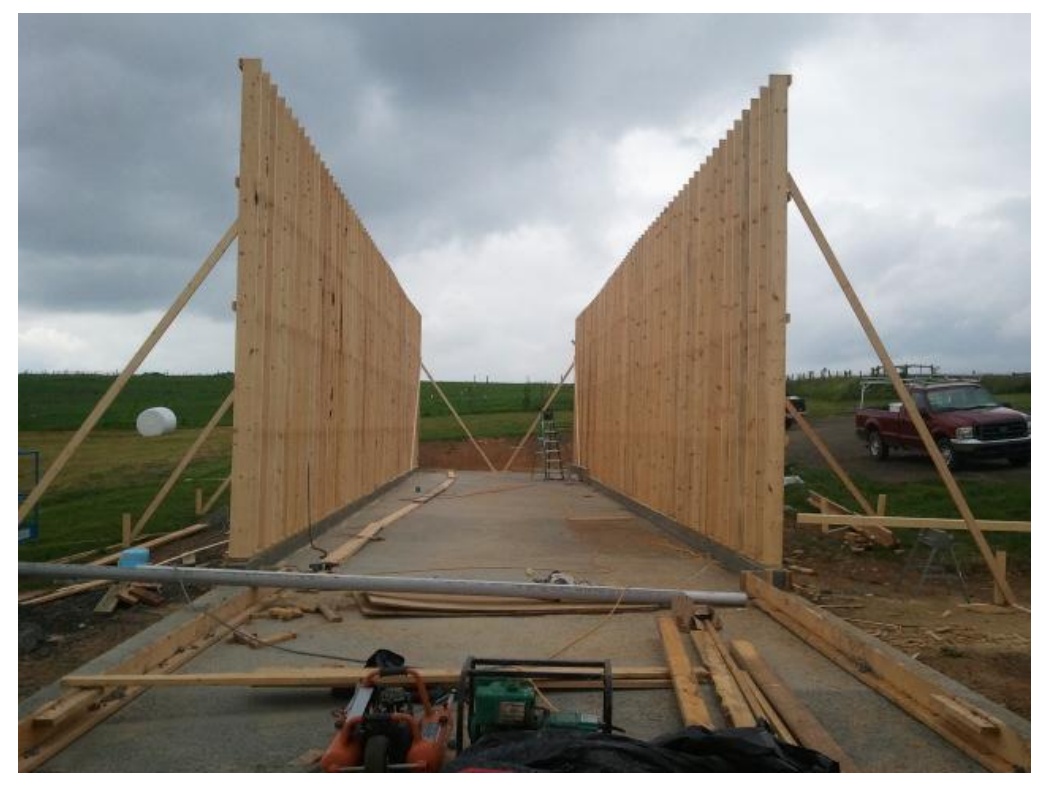

Figure 3-32: Wind tunnel wall installation at the construction site 


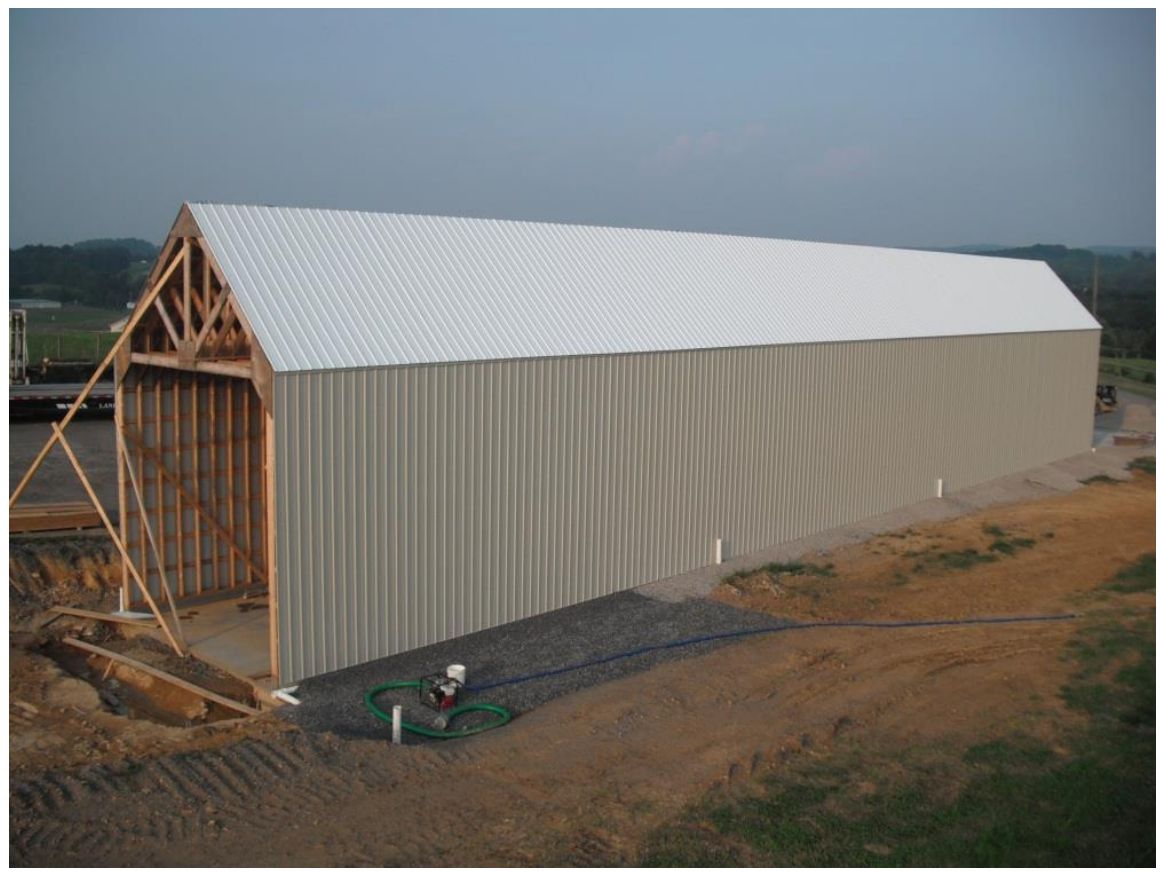

Figure 3-33: Overview of the finished main wood structure, metal siding, and roofing

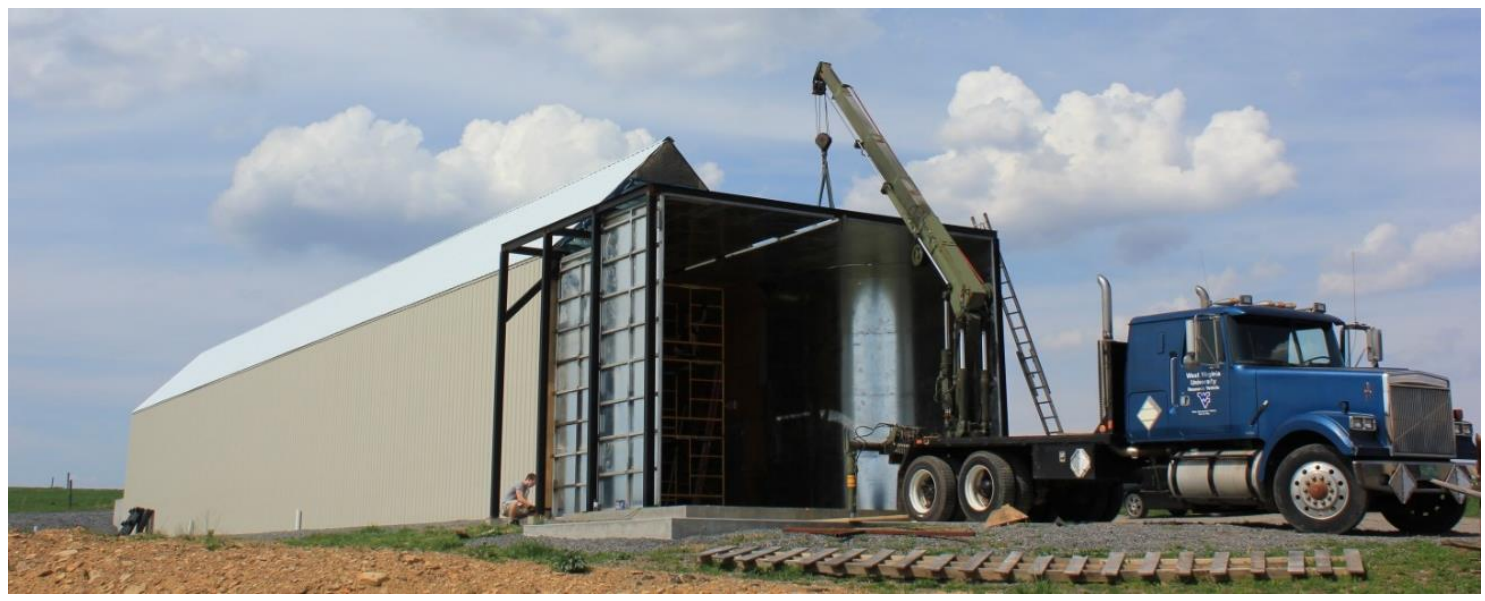

Figure 3-34: Overview of the installation of the main metal structure 
Due to the unavailability of electrical power in excess of $1 \mathrm{Mw}$ from the local power lines, the author recognized a diesel engine as the best solution for powering the propeller of the wind tunnel. Based on the wind tunnel power requirement of $1200 \mathrm{HP}$, a $2200 \mathrm{bHP}$ diesel engine was chosen (see Table 3-4). Figure 3-35 shows the final installation of the engine.

Table 3-4: Engine Propeller Specifications

\begin{tabular}{lc}
\hline Parameter & Value \\
\hline Engine Manufacturer & Detroit Diesel Corp. \\
\hline Model & 16v149TiB \\
\hline Number of Cylinders & 16 \\
\hline Cylinders Configuration & $\mathrm{V}$ \\
\hline Displacement [L] & 39.18 \\
\hline Max Power[BHP@rpm] & $2200 @ 1900$ \\
\hline Max Torque [lb-ft@rpm] & $6683 @ 1350$ \\
\hline
\end{tabular}

The propeller chosen is produced by Whirl Wind Propellers Corporation, and Table 3-5 summarizes its main characteristics. Actual noise level for this propeller is not available but, it is available the noise level for a propeller with $3 / 4$ diameter. Considering that both have similar blade tip velocities, it is reasonable to assume comparable noise levels. 


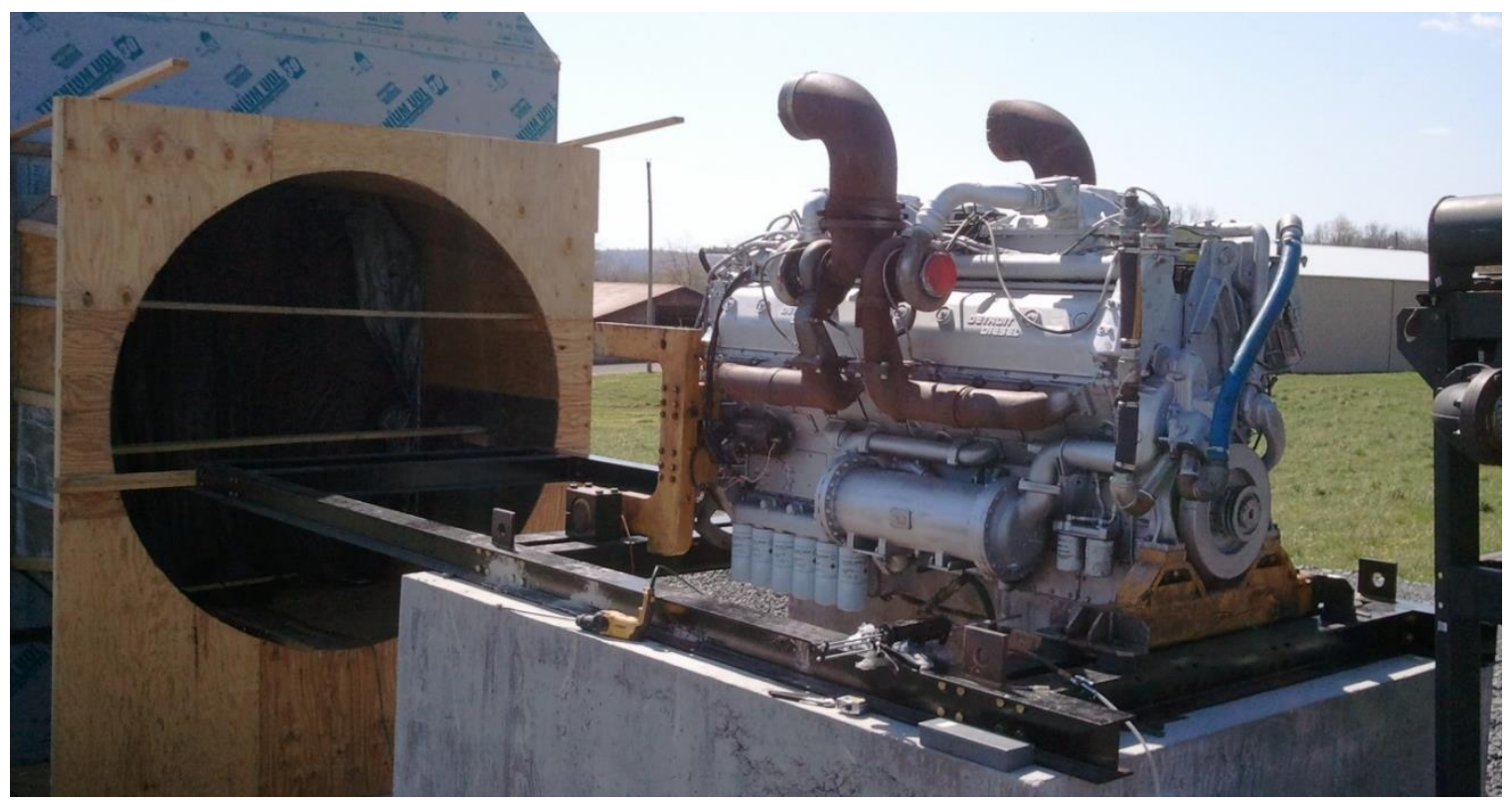

Figure 3-35: Installation of the propeller engine on its final setup

Table 3-5: Wind Tunnel Propeller Specifications

\begin{tabular}{lc}
\hline Parameter & Value \\
\hline Manufacturer & Whirl Wind Propellers \\
\hline Model & Wind Tunnel \\
\hline Blade Material & Carbon Fiber \\
\hline Number of blades & 5 \\
\hline Hub Material & Aluminum \\
\hline Diameter [ft.] & 10 \\
\hline Max Power [HP] & 1500 \\
\hline Max Rotational Speed [rpm] & 1500 \\
\hline Noise Level [db@ft] & $80 @ 50$ \\
\hline
\end{tabular}


The fundamental auxiliary service needed to support wind tunnel operation is electrically compressed air. An electricity service value of approximately 200 amps, 120/240V, (single phase) will be needed for tunnel lighting, sampling systems, data acquisition, and control. In order to meet the accelerated timeline of the current project, generator power will be provided. Compressed air will be provided from a dedicated compressor with moisture and particulate removal. The variety of tests that are going to be performed, and the associated types of measurement, require a large number of instruments and significant data management systems. A customized data acquisition (DAQ), specially developed by Center for Alternative Fuels Engines and Emissions (CAFEE), was utilized. It is able to acquire the data from emissions instruments (gaseous and particulate matter), placed in different positions, as well as engine sensors and wind tunnel control. Such DAQ acquires contemporaneously from all the instruments, avoiding the time alignment issues and producing a single collection data file. The data capturing adopts $10 \mathrm{hz}$, meeting the 1hz minimum required indicated by Title 40 CFR part 1065 .

\subsection{WIND TUNNEL QUALIFICATION AND COMMISSIONING}

The qualification procedure investigates the quality of the flow generated in the empty wind tunnel. The core parameters for this analysis are wind speed and TI on different cross section planes. Three planes are identified in the tunnel for these measurements, with respective longitudinal distances of $0 \mathrm{~m}, 15 \mathrm{~m}$, and $30 \mathrm{~m}$. The planes with coordinates $0 \mathrm{~m}$ and $30 \mathrm{~m}$ are the most forward and most rear sections of the wind tunnel, while the $15 \mathrm{~m}$ plane is placed halfway through the tunnel. A total of 25 sampling points per plane are chosen, with a sampling time of 100 seconds each at $10 \mathrm{~Hz}$. 
Results indicate that for the $35 \mathrm{mph}$ wind speed (highest flow energy content) the requirement of homogenous flow and TI $<1.5 \%$ are met. In detail, Table 3-6 and Figure 336 show the variation between the core and the outer region of the flow (closer to the walls); and an increasing variation is observed from the inlet to the outlet of the test section, from $3.1 \%$ to $3.7 \%$. The results are defined satisfactory and fall within the range indicated by Rae, William, \& Pope (1984) of 5\%.

Table 3-6: Wind Speed Variation Between the Core and Outer Region for the Qualification Test at 35mph Sind Speed.

\begin{tabular}{|lc|}
\hline Location & Variation [\%] \\
\hline Plane at $\mathbf{0 m}$ & 3.1 \\
\hline Plane at $\mathbf{1 5 m}$ & 3.3 \\
\hline Plane at $\mathbf{3 0 m}$ & 3.7 \\
\hline
\end{tabular}




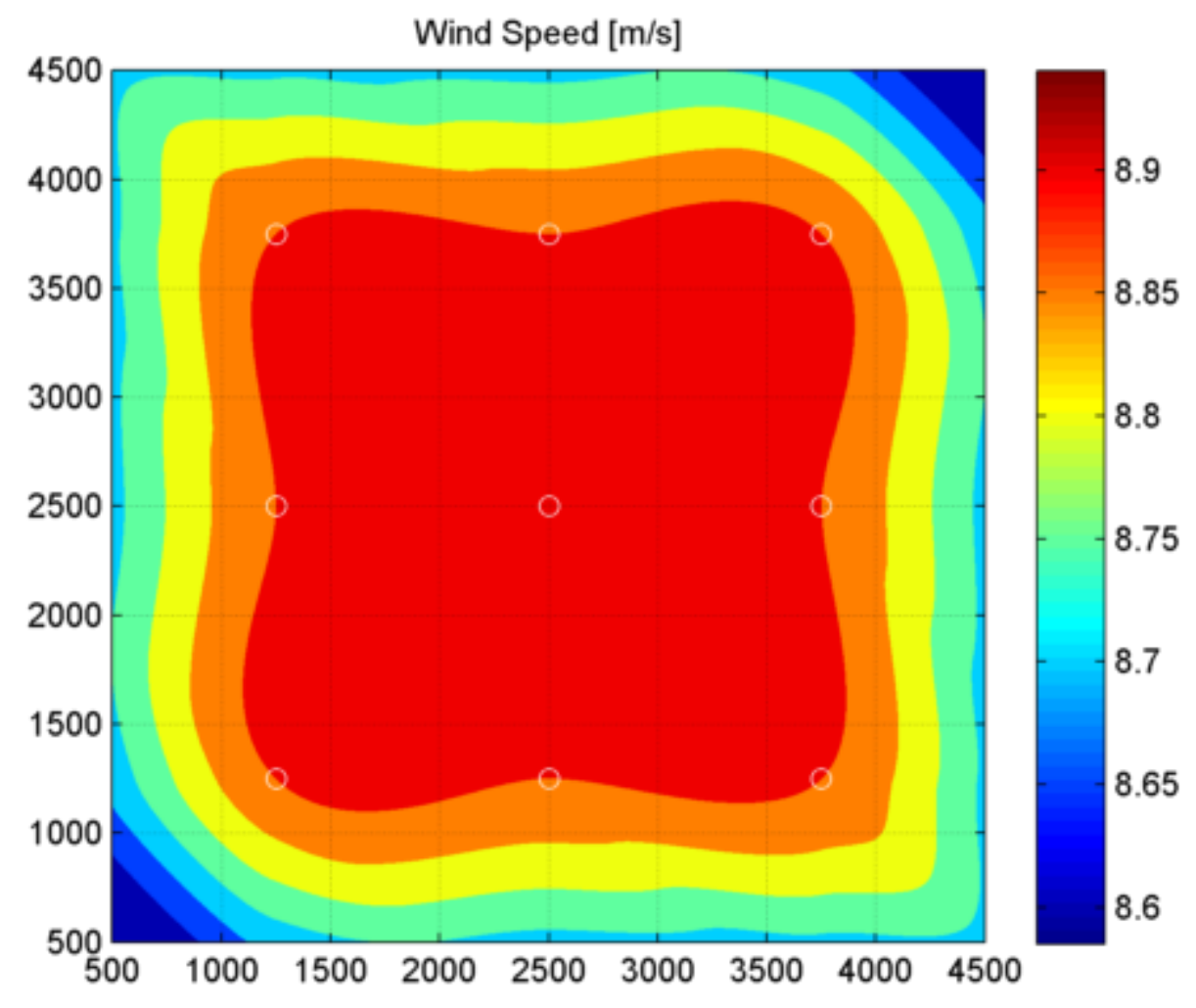

Figure 3-36: Wind speed measurements during the qualification test at $35 \mathrm{mph}$ at the $15 \mathrm{~m}$ plane. The white circles represent the sampling points. Vertical and horizontal dimensions are in $\mathrm{mm}$

TI intensity measurements indicate almost constant values across the section with $1.05 \%$ as the highest value, wherein the distance doesn't play a significant role. 


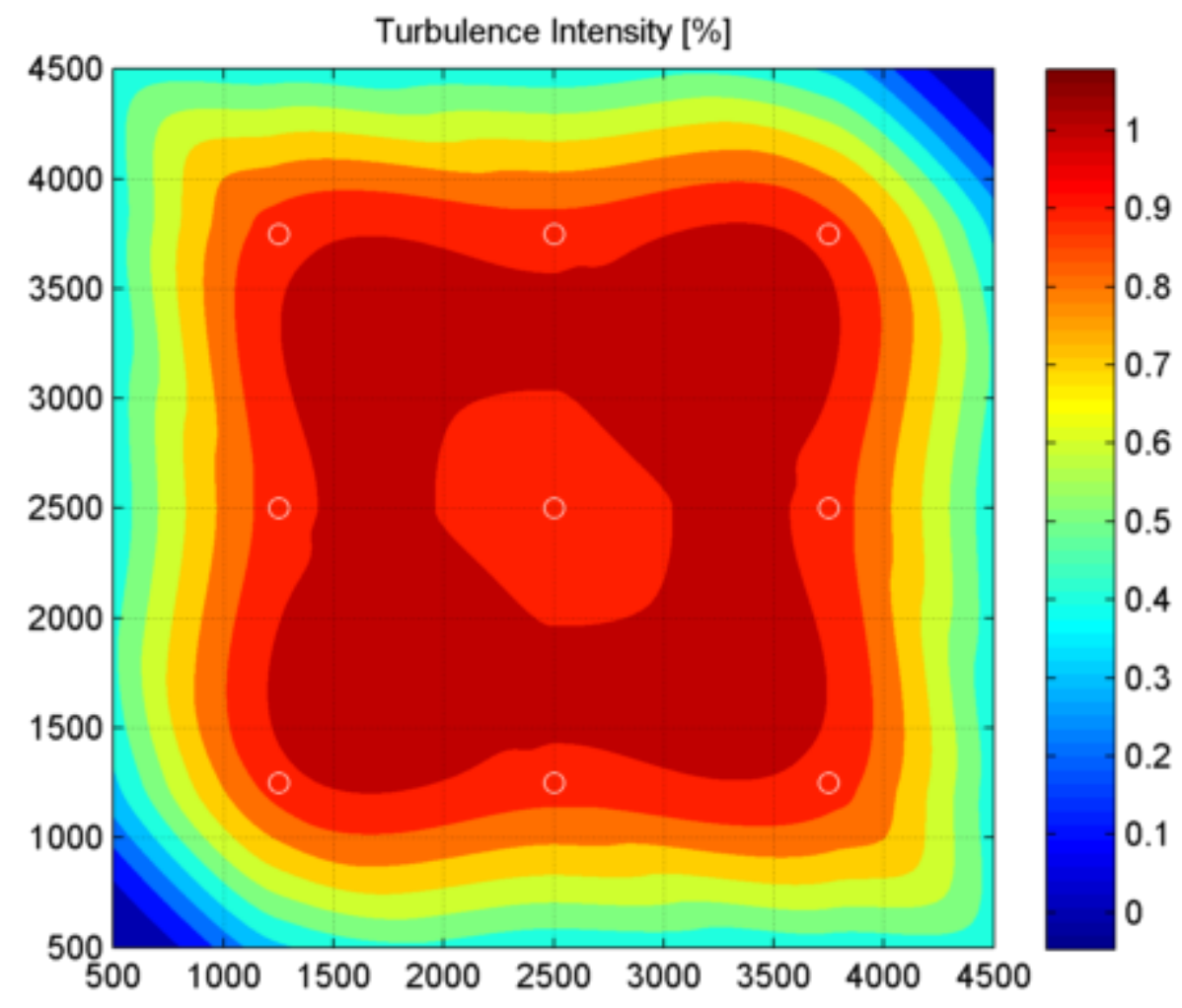

Figure 3-37: TI measurements during the qualification test at $35 \mathrm{mph}$ at the $15 \mathrm{~m}$ plane. The white circles represent the sampling points. Vertical and horizontal dimensions are in $\mathrm{mm}$ 


\section{Chapter 4: Experimental Methodology}

\subsection{Instruments, Sampling Procedures, ANd QA/QC}

In order to supply a continuous stream of exhaust for the exhaust plume simulation within the tunnel, a vehicle is installed on a heavy-duty chassis dynamometer, located outside the wind tunnel, and operated at a pre-defined vehicle speed. The vehicle's exhaust stack is connected to a 5 in (diameter) transfer pipe and ducted through an underground channel to a mock-up tractor cabin located inside the wind tunnel (see Figure 4-2). The position of the chassis dynamometer outside the wind tunnel is in close proximity to the position of exhaust introduction; this combined with the thermal insulation of the transfer pipe, guarantees a minimal temperature and PM loss of the exhaust stream. The results shows a temperature drop smaller than $50^{\circ} \mathrm{C}$ and a residence time smaller than one second. This solution was chosen to avoid the logistical and structural problems of placing the chassis dynamometer inside the wind tunnel.

It should be noted that this specific issue only arises for plume studies within the WVU wind tunnel. The heavy-duty chassis dynamometer employed during the presented study is part of WVU's Transportable Emissions Measurement System (TEMS). The chassis dynamometer, built by West Virginia University, consists of two sets of 12.6in diameter rollers, two flywheel assemblies, two air cooled eddy current power absorbers (Model CC300, Mustang) capable of absorbing peak power up to 1000hp, and two electrical motors to balance frictional losses within the dynamometer system, one for each 
side (hub) of the vehicle's drive axle. The flywheel assembly simulates the inertia of the test vehicle and is capable of simulating a vehicle weight of up to $110,000 \mathrm{lbs}$.

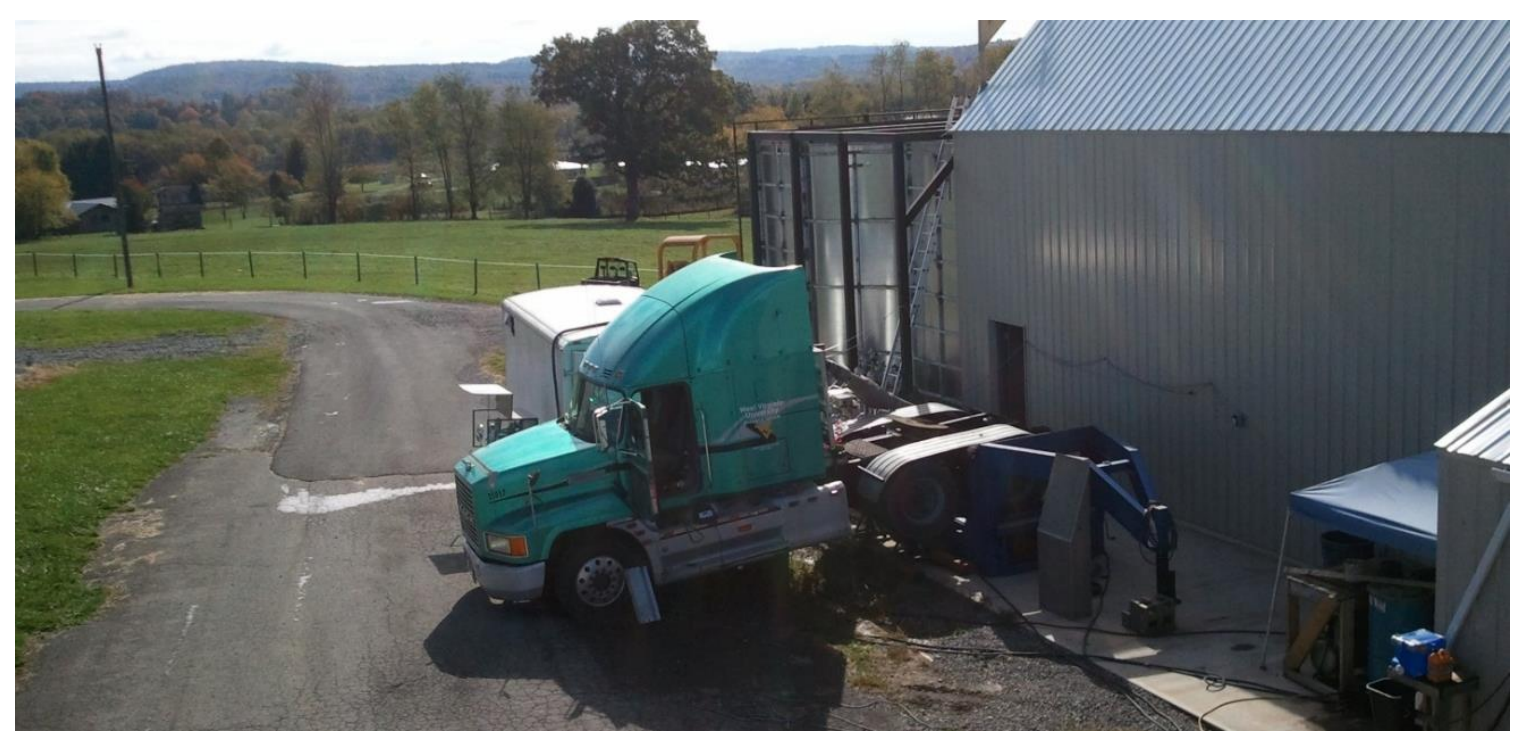

Figure 4-1: Wind tunnel, chassis dynamometer and TREL setup

Recalling the concept of flexibility, which the WVU wind tunnel has been designed upon, the author would like to highlight a possible setup configuration allowing for studying the impact of aerodynamic effects onto exhaust emissions. For such a scenario, the WVU wind tunnel would be transformed into a blowing configuration by simply reversing the tunnel flow. A concrete platform located at a slightly lower level just in front of the regular wind tunnel inlet would accommodate the chassis dynamometer, so that the installed test vehicle will be flush with the floor of the wind tunnel itself. This study could aid in understanding how the external aerodynamics of a vehicle affect its emissions. 


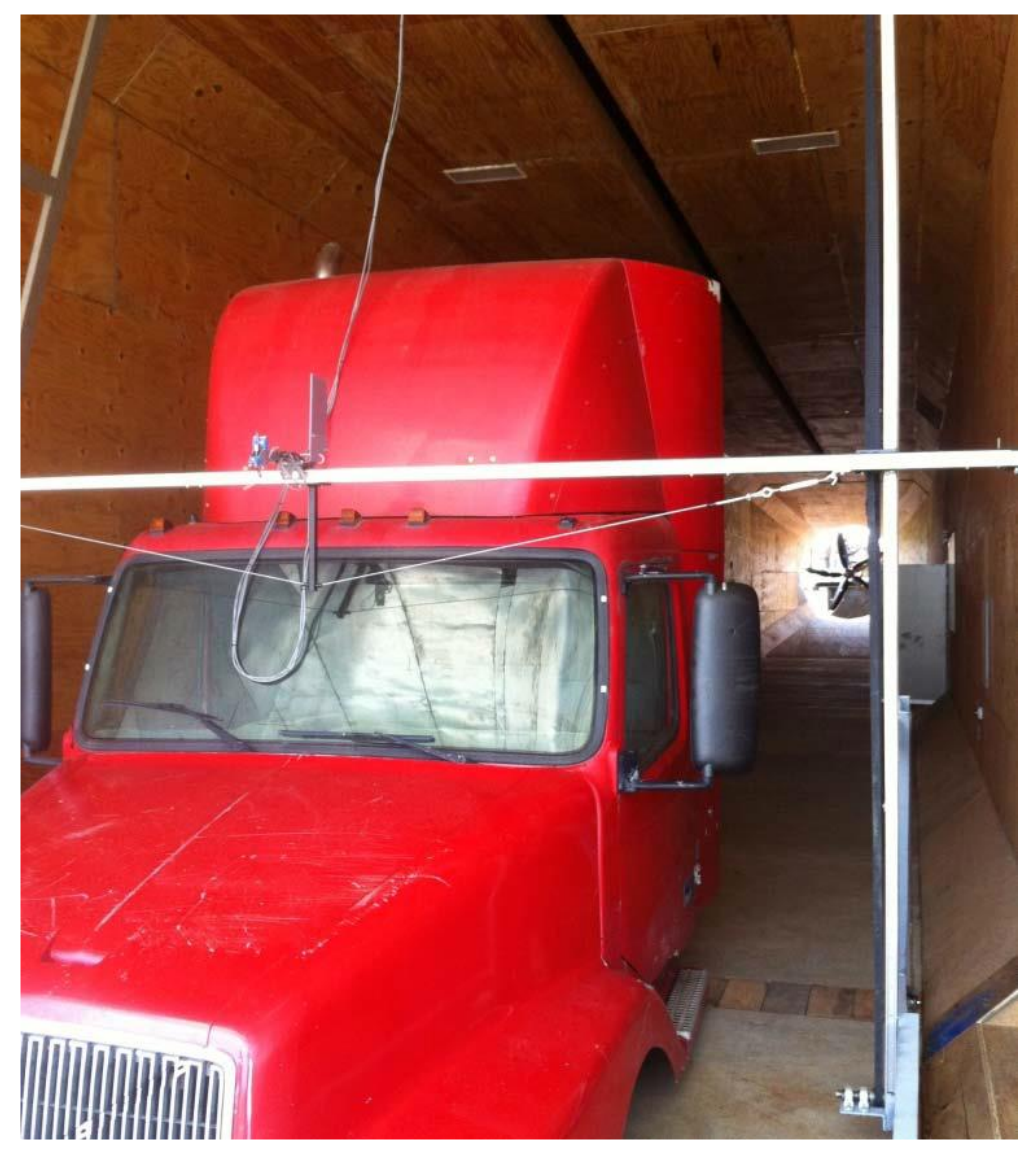

Figure 4-2: Internal view of the wind tunnel with mock-up truck and sampling gantry

The instrumentation setup during a primary exhaust plume study is composed of three different sampling stations able to identify the three main flows involved. Continuous data from all three sampling stations is acquired at a sampling rate of $10 \mathrm{~Hz}$ using a custom designed data acquisition system developed by CAFEE. Furthermore, the acquisition system is linked to an integrated system command computer, allowing for real-time control of the propeller (via DDC engine control), instrumentation cart, and sampling probe as well as the chassis dynamometer. 


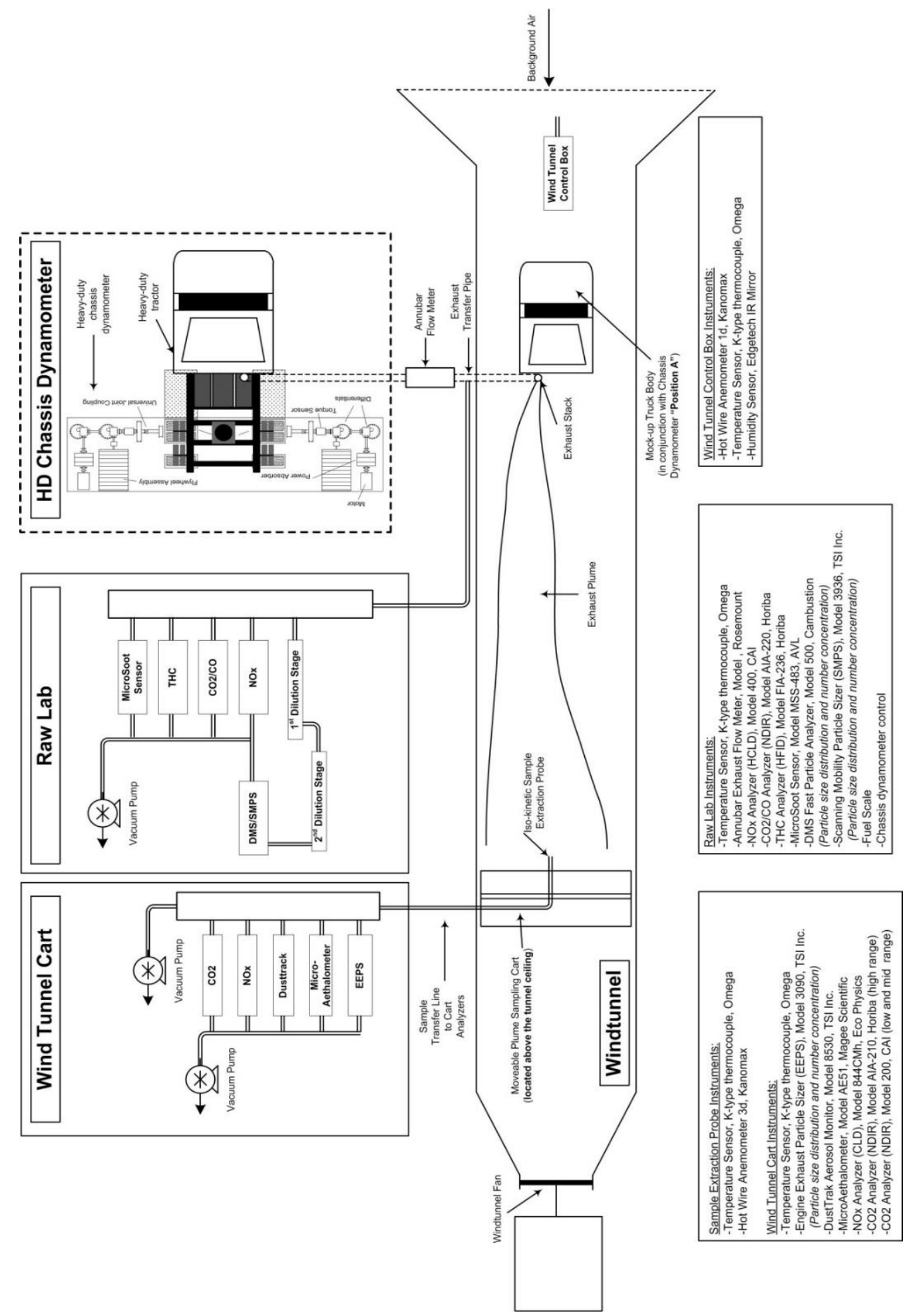

Figure 4-3: Experimental setup schematic 


\subsubsection{Plume Instruments}

The one of utmost importance to this particular study is the mobile platform, termed instrumentation cart for the reminder of the report, which analyzes the exhaust plume. The instrumentation cart is equipped with following analytical instruments:

Sample Extraction Probe Instruments:

- Temperature Sensor, K-type thermocouple, Omega

- Hot Wire Anemometer 3d, model 3080, Kanomax

Wind Tunnel Sampling Cart Instruments:

- Temperature Sensor, K-type thermocouple, Omega

- Engine Exhaust Particle Sizer ${ }^{\mathrm{TM}}\left(\right.$ EEPS $\left.^{\mathrm{TM}}\right)$, Model 3090, TSI Inc.

(Particle size distribution and number concentration)

- DustTrak $^{\text {TM }}$ Aerosol Monitor, Model 8530, TSI Inc.

- MicroAethalometer, Model AE51, Magee Scientific

- $\quad \mathrm{NO}_{\mathrm{x}}$ Analyzer (CLD), Model 844CMh, Eco Physics

- $\quad \mathrm{NO}_{\mathrm{x}}$-amines Analyzer (CLD), Model 844CMh, Eco Physics

- $\mathrm{CO}_{2}$ Analyzer (NDIR), Model AIA-210, Horiba (high-range)

- $\mathrm{CO}_{2}$ Analyzer (NDIR), Model 200, CAI (low- and mid-range)

Carbon dioxide $\left(\mathrm{CO}_{2}\right)$ (low-, mid- and high-range) is measured on a dry basis using a non-dispersive infrared (NDIR) analyzer (Model AIA-220, Horiba and Model 200, CAI). A solution with a combination of three different $\mathrm{CO}_{2}$ analyzers was chosen to better cover 
the wide range of measurements, from 450ppm in the background air, to $10 \%$ in the core of the plume. The ranges of the three instruments overlap, thus careful attention needs to be put in place to evaluate the most reasonable local value of $\mathrm{CO}_{2}$. Oxides of nitrogen $\left(\mathrm{NO}_{\mathrm{x}}\right)$ are measured on a wet basis using a Chemiluminescence detector (CLD) (Model 844CMh, Eco Physics). All analyzers are calibrated and operated according to recommendations outlined in the Code of Federal Regulations (CFR), title 40, and part 1065.

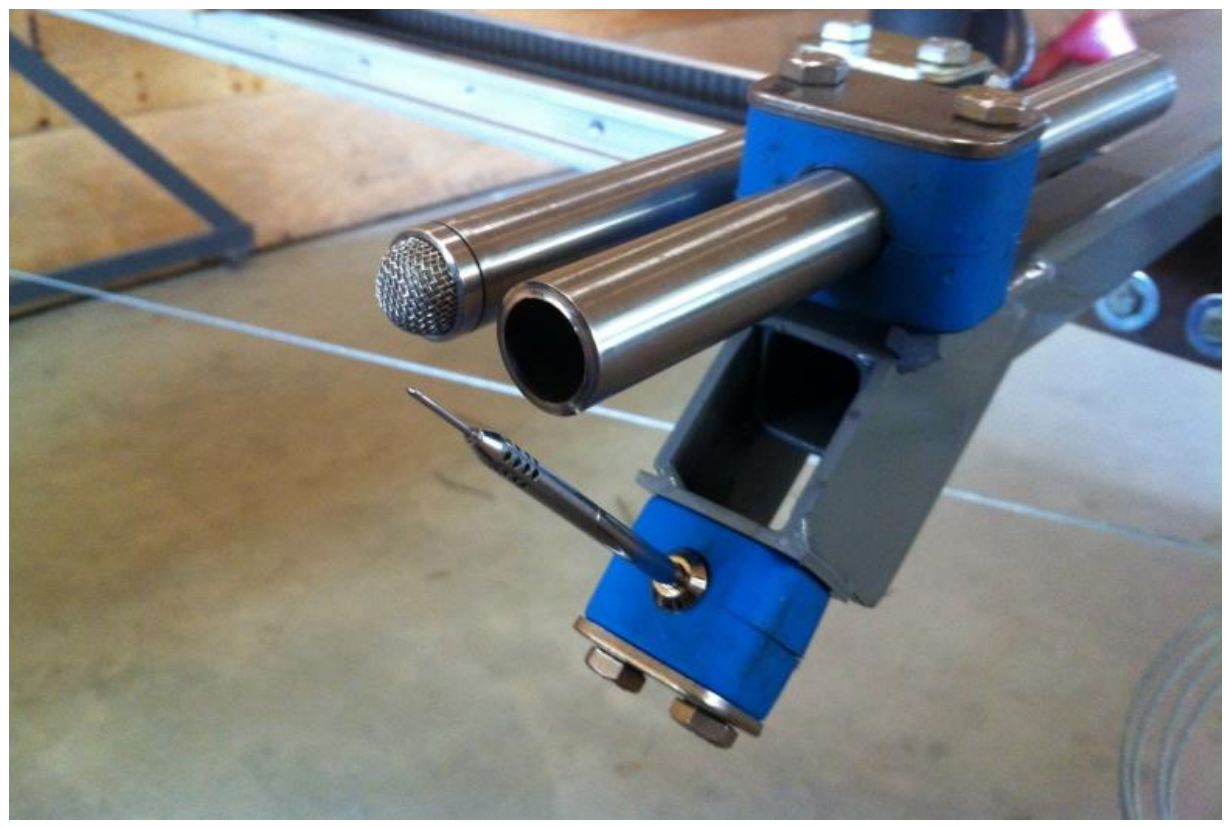

Figure 4-4: Exhaust plume sampling probe and flow field characterization sensors 
An amines converter using a Chemiluminescence detector (CLD) (Model 844CMh, Eco Physics) provides the combination signal of $\mathrm{NO}_{\mathrm{x}}$ and ammonia. Ammonia can be evaluated by subtracting the $\mathrm{NO}_{\mathrm{x}}$ signal from the $\mathrm{NO}_{\mathrm{x}}$-amines. The flow in the sample extraction probe is managed according to the local wind speed (hot wire anemometer right in front of the probe tip), achieving in every point, an isokinetic sampling probe. This solution was chosen to reduce the particle losses and sample the region as big as the probe tip, avoiding averaging measurements. The sample flow had no effects on the wind speed measurements obtained by the hot wire anemometer. The span gases utilized conform to the following:

Pre-2007 and Post-2007 Truck:

Table 4-1: Span Calibration Gases for the Sampling Cart Gaseous Instruments for Pre-2007 and Post-2007 Truck

\begin{tabular}{lc}
\hline Gaseous Analyzer & Span Calibration Gas \\
\hline CO2 low-range Analyzer & $0.25 \%$ \\
\hline $\mathrm{CO}_{2}$ mid-range Analyzer & $1 \%$ \\
\hline $\mathrm{CO}_{2}$ high-range Analyzer & $8.139 \%$ \\
NOx Analyzer & $1000 \mathrm{ppm}$ \\
\hline Total-amines Analyzer & $1000 \mathrm{ppm}$ \\
\hline
\end{tabular}


Post-2010 Truck:

Table 4-2: Span Calibration Gases for the Sampling Cart Gaseous Instruments for Post2010 Truck

\begin{tabular}{lc}
\hline Gaseous Analyzer & Span Calibration Gas \\
\hline $\mathbf{C O}_{2}$ low-range Analyzer & $0.25 \%$ \\
\hline $\mathbf{C O}_{2}$ mid-range Analyzer & $1 \%$ \\
$\mathbf{C O}_{2}$ high-range Analyzer & $8.139 \%$ \\
\hline $\mathbf{N O}_{\mathbf{x}}$ Analyzer & $148.9 \mathrm{ppm}$ \\
\hline Total-amines Analyzer & $148.9 \mathrm{ppm}$ \\
\hline
\end{tabular}

Measurements of PM concentrations and size distributions are performed with an Engine Exhaust Particle Sizer (EEPS ${ }^{\mathrm{TM}}$ ) spectrometer, model 3090, from TSI Inc. (Minnesota, USA) within the exhaust plume and the background air. Spectrometers classify particles based on the differential electrical mobility classification principle at a frequency of $1 \mathrm{~Hz}$, making it best suited for transient PM characterization. Particles between $6.04 \mathrm{~nm}$ and $523.3 \mathrm{~nm}$ are measured with a resolution of 16 channels per decade; hence a total of 32 channels.

The DustTrak ${ }^{\mathrm{TM}}$ Aerosol Monitor (Model 8530, TSI Inc.) is a light-scattering laser photometer capable of measuring suspended aerosol mass corresponding to $\mathrm{PM}_{2.5}$. The DustTrak $^{\mathrm{TM}}$ came pre-calibrated from the California Air Resource Board (CARB) (owner of the instruments). Prior to any tests, the instruments were cleaned and zeroed following the procedures indicated in the manuals. The linear response of the instruments and the 
zeroing performed prior to any tests prevented sensible drift and offset in the measurements.

MicroAethalometer (Model AE51, Magee Scientific) is a laser absorption photometer capable of measuring black carbon concentrations. Engine Exhaust Particle Sizer (EEPSTM) (Model 3090, TSI Inc.), DustTrak ${ }^{\mathrm{TM}}$ Aerosol Monitor (Model 8530, TSI Inc.) and MicroAethalometer (Model AE51, Magee Scientific) are operated according to their manuals and periodic cleaning is enforced prior to any test. The transfer line from the probe to the analyzers is a Tygon ${ }^{\mathrm{TM}}$ tube that is $6 \mathrm{~m}$ long; it is kept thermally isolated with heating and insulation tape at $60^{\circ} \mathrm{C}$ to reduce thermophoretic losses (predominant) and not influence the PM particle and size distribution. The material of the sampling line is black Tygon $^{\mathrm{TM}}$, which guarantees low electrostatic losses (Liu, Pui, Rubow, \& Szymanski, 1985).

An Electrospray Aerosol Generator (EAG) Model 3480 from TSI was used to identify the PM particle penetration efficiency through the sampling line. Figure 4-5 shows the penetration efficiency per particle size with a spectrum wider than typical Diesel PM. A penetration higher than $90 \%$ for particles greater than $10 \mathrm{~nm}$ represents a desirable result for a long sampling line, guaranteeing accurate investigation of the plume. 


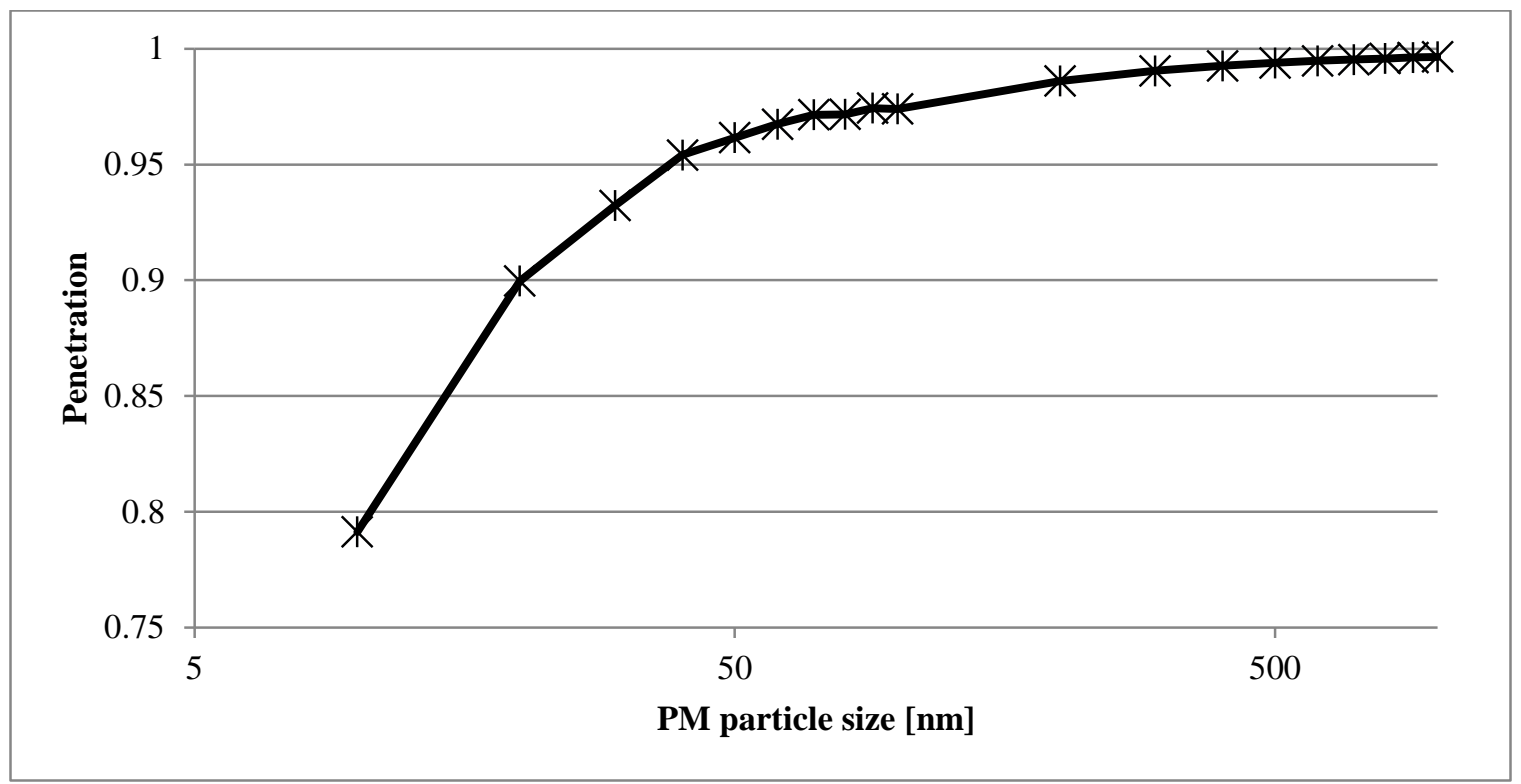

Figure 4-5: PM penetration efficiency for the heated sampling line at a flow rate of 5slpm for a particle size spectrum wider than typical diesel PM

Isokinetic sampling requires a sampling flow rate that spans from 0.5 to $5 \mathrm{slpm}$. While results in Figure 4-5 are obtained with a flow rate of $5 \mathrm{scfm}$, it is noticed that the penetration is not affected by the sampling flow or the inlet temperature of the sample. In addition, results indicate no trace of artifact generation in the sampling line. 


\subsubsection{Raw Exhaust Instruments}

The second sampling station measures the raw exhaust directly from the truck's exhaust stack.

Transportable Raw Emissions Laboratory (TREL):

- Temperature Sensor, K-type thermocouple, Omega

- 4in. Annubar Exhaust Flow Meter, Rosemount

- $\quad \mathrm{NO}_{\mathrm{x}}$ Analyzer (CLD), Model 400, CAI

- $\mathrm{CO}_{2} / \mathrm{CO}$ Analyzer (NDIR), Model AIA-220, Horiba

- $\quad$ THC Analyzer (HFID), Model FIA-236, Horiba

- MicroSoot Sensor, Model MSS-483, AVL

- Double Dilution Stage System:

- DMS Fast Particle Analyzer, Model 500, Cambustion

(Particle size distribution and number concentration)

○ Scanning Mobility Particle $\operatorname{Sizer}^{\mathrm{TM}}\left(\right.$ SMPS $\left.^{\text {тм}}\right)$, Model 3080, TSI Inc.

(Particle size distribution and number concentration)

- Fuel Scale

- Chassis eddy current dynamometer power absorber, Model CC300, Mustang

- Chassis dynamometer Dyn-Loc IV digital controller 
The raw exhaust platform comprises WVU's Transportable Raw Emissions Laboratory (TREL) shown in Figure 4-6, which was designed and constructed according to requirements outlined in CFR, title 40, part 1065, and capable of characterizing gaseous emissions of $\mathrm{CO}_{2}, \mathrm{CO}, \mathrm{NO}_{\mathrm{x}}, \mathrm{NO}$ as well as THC from raw (undiluted) exhaust. $\mathrm{NO}_{\mathrm{x}}$ and NO are measured on a wet basis using a dual-channel CLD (Model 844CMh, Eco Physics) with the possibility to calculate $\mathrm{NO}_{2}$ through subtraction of $\mathrm{NO}$ from $\mathrm{NO}_{\mathrm{x}}, \mathrm{CO}_{2}$ and $\mathrm{CO}$ on a dry basis using an NDIR analyzer (Model AIA-220, Horiba); as well as THC on a wet basis using a Hydrocarbon flame ionization detector (HFID) analyzer (Model FIA-236, Horiba).

The analytical bench further houses sampling conditioning units (dryers), flow meters, and heated sampling pumps and filters that are controlled by electronic microcontrollers and maintained at $112^{\circ} \mathrm{C}$ for $\mathrm{CO}, \mathrm{CO}_{2}$ and $\mathrm{NO}_{\mathrm{x}}$; and $191^{\circ} \mathrm{C}$ for $\mathrm{THC}$ sample trains. Furthermore, TREL includes a partial-flow PM sampling system, not used for this test campaign, and BG-3 from Sierra, allowing for PM sample collection on filter media (TX40, Pallflex ${ }^{\circledR}$ ) for subsequent gravimetric weighing or elemental carbon / organic carbon (EC/OC) analysis using pre-fired quartz fiber filters. 


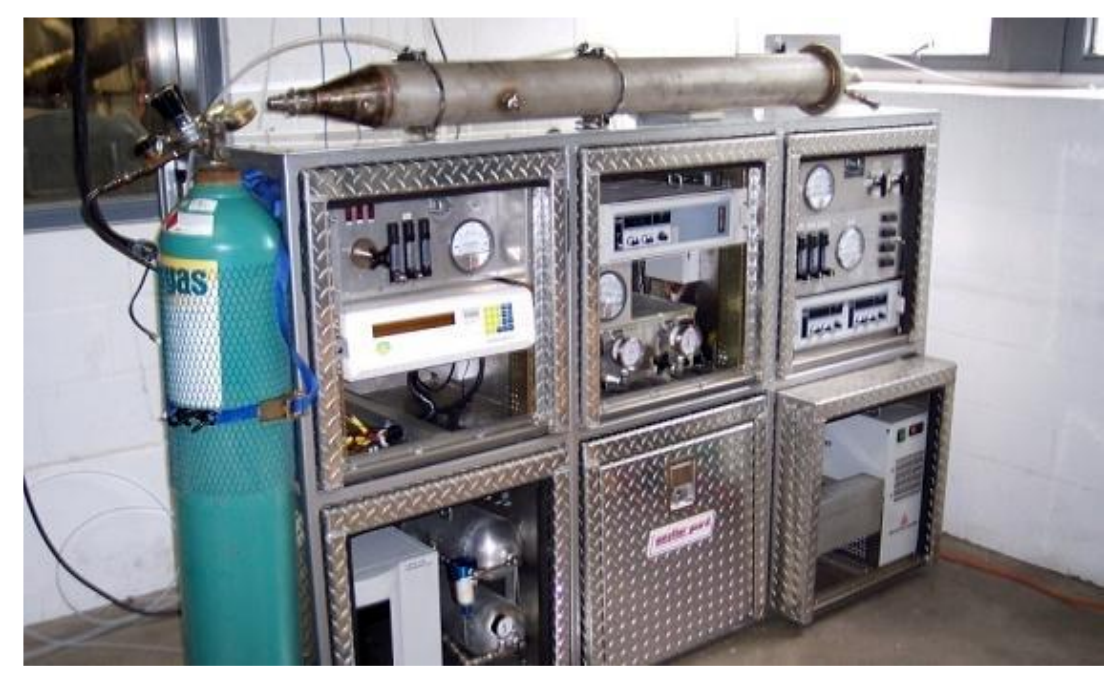

Figure 4-6: WVU Transportable Raw Emissions Laboratory (TREL)

Different aftertreatment technologies and devices allow for differences in terms of emissions measurement, leading to different calibration span values for analyzers.

$\underline{\text { Pre-2007 Truck }}$

Table 4-3: Span Calibration Gases for the TREL Gaseous Instruments for Pre-2007 Truck

\begin{tabular}{lc}
\hline Gaseous Analyzer & Span Calibration Gas \\
\hline CO2 Analyzer & $8.139 \%$ \\
\hline CO Analyzer & $1002 \mathrm{ppm}$ \\
THC Analyzer & $150.6 \mathrm{ppm}$ \\
\hline NOx Analyzer & $1000 \mathrm{ppm}$ \\
\hline
\end{tabular}


$\underline{\text { Post-2007 Truck }}$

Table 4-4: Span Calibration Gases for the TREL Gaseous Instruments for Post-2007 Truck

\begin{tabular}{lc}
\hline Gaseous Analyzer & Span Calibration Gas \\
\hline CO2 Analyzer & $8.139 \%$ \\
CO Analyzer & $100 \mathrm{ppm}$ \\
THC Analyzer & $25.6 \mathrm{ppm}$ \\
\hline NOx Analyzer & $1000 \mathrm{ppm}$ \\
\hline
\end{tabular}

$\underline{\text { Post-2010 Truck }}$

Table 4-5: Span Calibration Gases for the TREL Gaseous Instruments for Post-2010 Truck

\begin{tabular}{lc}
\hline Gaseous Analyzer & Span Calibration Gas \\
\hline CO2 Analyzer & $8.139 \%$ \\
CO Analyzer & $100 \mathrm{ppm}$ \\
THC Analyzer & $25.6 \mathrm{ppm}$ \\
\hline NOx Analyzer & $148.9 \mathrm{ppm}$ \\
\hline
\end{tabular}

Raw PM emissions were sampled from the exhaust transfer pipe by means of a customized Double Dilution System (DDS). The apparatus is composed of two minidilution tunnels equipped with two ejector diluters that allow for variable Dilution Ratio (DR) settings. 
The first stage of dilution was performed using hot air $\left(\sim 150^{\circ} \mathrm{C}\right)$ which was previously filtered and dried by means of a HEPA filter and a dryer. The second stage dilution was, on the contrary, obtained by supplying cold air $\left(\sim 20^{\circ} \mathrm{C}\right)$ in order to obtain a gas mixture with a temperature low enough to satisfy the requirements of the spectrometers used in this study. A double dilution composed by first stage hot dilution and a subsequent second stage cold dilution should prevent the volatile organic fraction (VOF) of PM from reaching supersaturation conditions during the dilution process, hence limiting the amount of particles due to the nucleation of VOF (nucleation mode). The PM spectrometers employed for particle count and size characterizations were:

- DMS Fast Particle Analyzer, Model 500, Cambustion (Particle size distribution and number concentration)

- $\quad$ Scanning Mobility Particle Sizer ${ }^{\text {тм }}\left(\right.$ SMPS $\left.^{\text {тм }}\right)$, Model 3080, TSI Inc. (Particle size distribution and number concentration)

The DMS can measure aerosols ranging from $5 \mathrm{~nm}$ to $2.5 \mu \mathrm{m}$. The fast response time, 200ms T10-90, of the differential mobility classifier allows for the capturing of fast changing spectra such as combustion emissions during transient engine conditions. The SMPS $^{\mathrm{TM}}$ measures the size distribution engaging electrical mobility detection technique. Particles, after passing through a charge neutralizer, enter an electric field where they are classified according to their electrical mobility. This classification occurs in the Differential Mobility Analyzer (DMA). For this study a long DMA (TSI, Model 3081) was used and particles were counted by an Ultrafine Condensation Particle Counter (UCPC) from TSI (Model 3025), set to the high-flow mode. The SMPSTM sampling flow rate 
(1.5lpm) was controlled with a critical orifice. The sheet flow rate was set 10 times higher (15lpm) and was provided by the internal dual-blower.

The SMPS ${ }^{\text {TM }}$ was programmed to scan particles over a size range of 5.73 to 216 $\mathrm{nm}$ across 64 channels per decade. Sampling time for the entire size range was $135 \mathrm{sec}$, with an up-and-down scan time of 120 and $15 \mathrm{sec}$, respectively. Several analyses confirmed the consistency of the data of the two instruments, over a wide range of operations. Several studies have shown that PM particle size distribution and concentration measured by EEPS $^{\mathrm{TM}}$, SMPS ${ }^{\mathrm{TM}}$, or DMS ${ }^{\mathrm{TM}}$ are in accordance with each other across a wide range of engine operations and exhaust aftertreatment setups (Zervas \& Dorlhène, 2006) (Ng, Ma, \& David, 2007).

Table 4-6: PM Spectrometer Used for the Different Tests

\begin{tabular}{ccc}
\hline Truck & Test & PM spectrometer \\
\hline Pre-2007 & Idle & DMS \\
& $20 \mathrm{mph}$ & DMS \\
& $35 \mathrm{mph}$ & SMPS \\
Post-2007 & Idle & DMS and SMPS \\
& $20 \mathrm{mph}$ & DMS and SMPS \\
& $35 \mathrm{mph}$ & DMS and SMPS \\
Post-2010 & Idle & SMPS \\
& $20 \mathrm{mph}$ & SMPS \\
& $35 \mathrm{mph}$ & SMPS \\
\hline
\end{tabular}


Additionally, a micro-soot sensor (MSS-483) from AVL was employed in order to measure continuous soot (solid carbon only) emissions from the raw exhaust stack.

\subsubsection{Background Air Instruments}

The third sampling station is placed at the inlet of the WVU wind tunnel, which continuously monitors background air properties; thus, allowing for subsequent correction of background contamination within the wind tunnel test section.

$\underline{\text { Wind Tunnel Control Box Instruments: }}$

- Hot Wire Anemometer 1D, Kanomax

- Temperature Sensor, K-type thermocouple, Omega

- Humidity Sensor, Edgetech IR Mirror

- Local Weather data from NOAA server

PM and gaseous component measurements of the background air are obtained with samplings outside the plume. 


\subsubsection{Transportable Heavy-Duty Vehicle Emissions Testing Laboratory (THDVETL)}

\section{Chassis Dynamometer}

The vehicles were tested on an inertial-based heavy-duty chassis dynamometer which is part of the West Virginia University's Transportable Heavy-Duty Vehicle Emissions Testing Laboratory (THDVETL). Built by WVU, the chassis dynamometer makes use of rotational inertia to reliably match actual transitional vehicle inertia at a wide range of speeds and Gross Vehicle Weights (GVW) up to 70,000lbs. The power output from the rear drive axle of the vehicle is transferred to flywheels, simulating the vehicle inertia, eddy current power absorbers, simulating the road load and Alternative Current (AC) variable speed motors to simulate light grade motoring, through the drive train units from hub adapters connected to the outboard wheels. The vehicle wheels run on 12.6in idler rollers which also insure that the left and right wheel speeds are the same by negating axle differential action.

Direct load coupling between the vehicle hub and dynamometer eliminates tireroller slippage, thereby reducing possible errors in speed data as well as the highly variable frictional loading at the tire-roller interface due to heat generation. During the test cycle, torque cells and speed transducers in the power absorber drivetrain measure vehicle load and speed. A Dyn-Loc IV digital dynamometer controller was integrated with the test system software to control the operation of the chassis dynamometer. 


\subsubsection{WVU Wind Tunnel}

The WVU wind tunnel design and construction were extensively described in the previous chapter. In this section, the technical aspect and the relative capabilities of the wind tunnel are going to be summarized (see Table 4-7). The WVU wind tunnel is a fullscale, open-circuit, suction tunnel. The open-circuit configuration is a key feature that guarantees fresh ambient background air flowing over the vehicle and therefore, constant dilution air composition and conditions for the entire test duration, while the suction configuration guarantees a homogenous flow field.

Table 4-7: WVU Wind Tunnel Specifications

\begin{tabular}{lc}
\hline Parameter & Value \\
\hline Configuration & Non-recirculating \\
\hline Propeller Configuration & Suction \\
\hline Test Cell Cross Section [ftxft] & $16 x 16$ \\
\hline Test Cell Length [ft] & 115 \\
\hline Max Wind Speed [mph] & 50 \\
\hline Turbulence Control System & ABS honeycomb \\
\hline Honeycomb mesh size [in. $x$ in.] & $0.25 \times 1.5$ \\
\hline Turbulence Intensity [\%] & $<1.5$ \\
\hline Position Accuracy Sampling System [mm] & $<5$ \\
\hline Max Load Instrument Cart [lbs.] & 1000 \\
\hline
\end{tabular}


The test section is $16 \mathrm{ft}(5 \mathrm{~m})$ high, $16 \mathrm{ft}(5 \mathrm{~m})$ wide, and $115 \mathrm{ft}(35 \mathrm{~m})$ long, thus capable of accommodating a full-scale class-8 HDDV with its trailer; matching as closely as possible real-world conditions. Air flow, up to $50 \mathrm{mph}$, through the wind tunnel is achieved by a 5-blade propeller (10ft diameter carbon fiber blades) with adjustable pitch coupled to a Detroit Diesel Corp. (DDC), model 16V149 engine, capable of providing up to $2200 \mathrm{hp}$.

The prime research interest of the WVU wind tunnel is focused on studying the dispersing exhaust plume behind the vehicle rather than the aerodynamic forces acting on it, which conventional wind tunnels are typically used for. This important difference is the rationale for the WVU wind tunnel to have a length scale of the test cell much greater than the actual test section, which allows the plume 2 seconds residence time at $35 \mathrm{mph}$ wind speeds, a time frame wide enough to capture the formation and evolution of the primary PM.

Table 4-8: Propeller Engine Specification

\begin{tabular}{lc}
\hline Parameter & Value \\
\hline Engine Manufacturer & Detroit Diesel Corp. \\
\hline Model & 16v149TiB \\
\hline Number of Cylinders & 16 \\
\hline Cylinders Configuration & $\mathrm{V}$ \\
\hline Displacement [L] & 39.18 \\
\hline Max Power[BHP@ rpm] & $2200 @ 1900$ \\
\hline Max Torque [lb-ft@ rpm] & $6683 @ 1350$ \\
\hline
\end{tabular}


The WVU wind tunnel can also be operated in reverse mode. In this case, the wind direction is reversed and the truck (not the mock-up) with the chassis dynamometer seat at the flow outlet (the flow entrance in normal conditions). This solution is not meant to study the plume, but to investigate the effect of wind on engines and aftertreatment systems, and consequently on raw emissions.

Table 4-9: Wind Tunnel Propeller Specifications

\begin{tabular}{lc}
\hline Parameter & Value \\
\hline Manufacturer & Whirl Wind Propellers \\
\hline Model & Wind Tunnel \\
\hline Blade Material & Carbon Fiber \\
\hline Number of blades & 5 \\
\hline Hub Material & Aluminum \\
\hline Diameter [ft] & 10 \\
\hline Max Power [HP] & 1500 \\
\hline Max Rotational Speed [rpm] & 1500 \\
\hline Noise Level [db@ft] & $80 @ 50$ \\
\hline
\end{tabular}




\subsubsection{Sampling Plume Pattern}

The will of a detailed plume investigation and the guarantee for almost constant boundary conditions during the test made the author decide to not exceed six hours for each combination of truck and wind speed. The adopted solution was comprised of 130 sampling points divided into ten planes. Each plane presents the same sampling pattern, with different vertical dimensions and positions, better focusing on the plume shape and dimension evolution. The pattern is composed of two nested hexagons, rotated by $90^{\circ}$, and an additional center point. This configuration presents the best trade off in terms of matching the plume with a low number of sampling points per plane (see Figure 4-7). The original 130 points and additional p0 (point 0 ) are added right at the stack exhaust, intended to compare drifts or shifts of the sampling cart analyzers with the ones of the TREL. The first 65 points and p0 present 100s sampling time (1000 data points), and the remaining points 50s (500 data points). This solution allows for better investigation of turbulence in the area closest to the stack exhaust, where the highly turbulent kinetic energy of the flow represents the predominant driver for plume mixing and evolution.

Far away from the exhaust stack, turbulent mixing has lost most of its energy, and a slow gas diffusion process takes place. Prior to any sampling, 30s of non-sampling stabilization time is observed once the probe reaches the desired position, accounting for the inertia of the measurement system. The definition process for the point coordinates started with Figure 4-8, a preliminary plume simulation, and overlapped with the wind tunnel CAD model. Preliminary tests further refined the sampling point coordinates. 


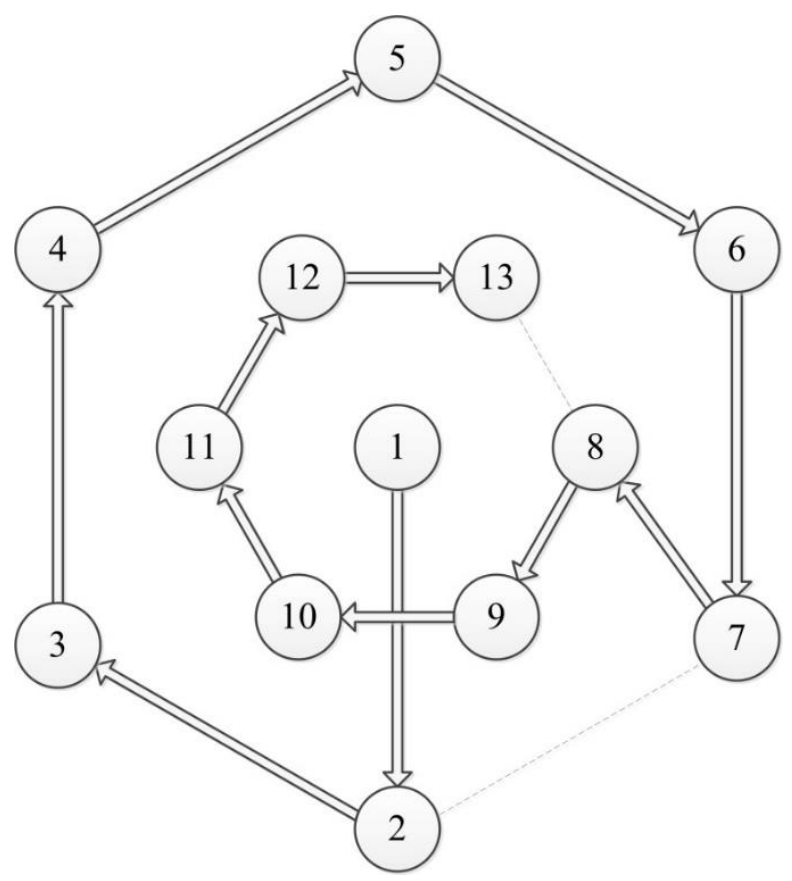

Figure 4-7: Sampling plume pattern for each plane and the path followed by the sampling probe

In the end, three different sampling pattern sets are identified, one per each wind speed. The three trucks involved in this test campaign show similar exhaust flow rates, thus making it unnecessary to adapt the sampling pattern set to the trucks. The three different sampling pattern sets share the vertical dimension of the sampling pattern (distance between point 2 and 5 of Figure 4-7) and the distance between the sampling planes and the exhaust stack; while the main difference is related to the vertical position of the sampling plane. Table 4-10 contains the common parameters of the different sampling pattern sets. 


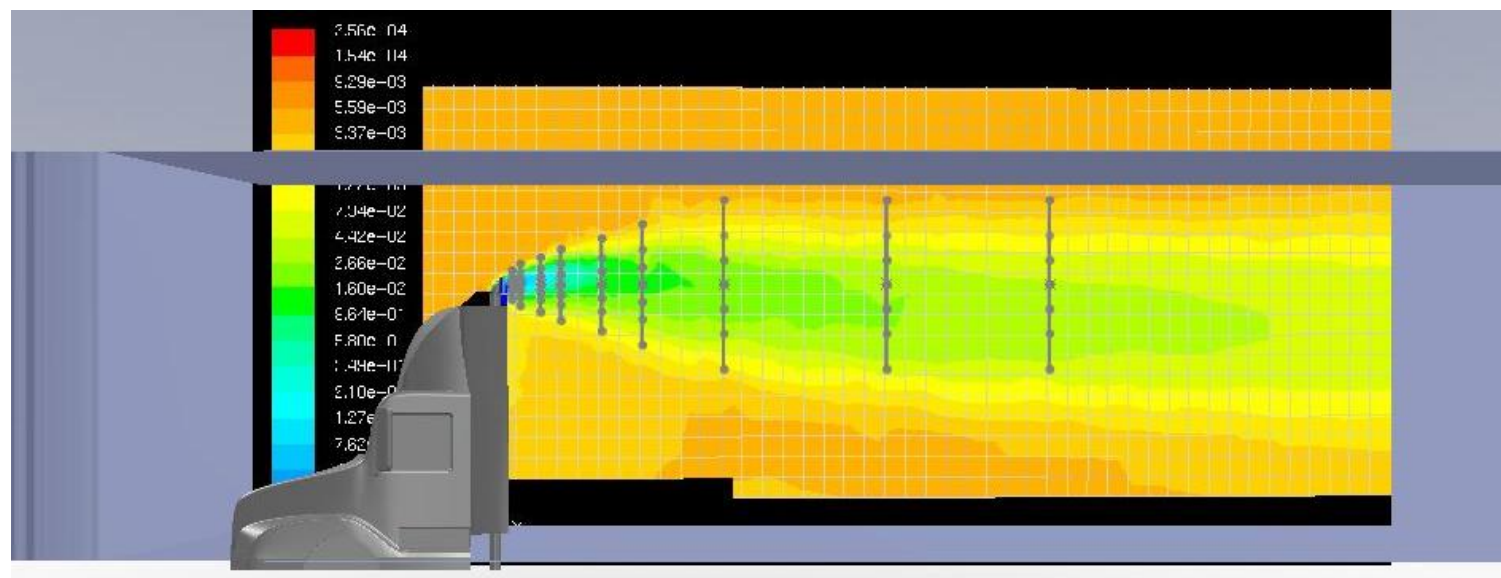

Figure 4-8: Initial identification of the plume sampling points

Table 4-10: Sampling Plume Pattern Parameters

Vertical Dim. [m]

Z Distance [m]

\begin{tabular}{|l|l|l}
\hline Plane1 & 0.350 & 0.115 \\
\hline Plane2 & 0.500 & 0.315 \\
\hline Plane3 & 0.600 & 0.565 \\
\hline Plane4 & 0.650 & 0.815 \\
\hline Plane5 & 0.700 & 1.315 \\
\hline Plane6 & 0.800 & 1.815 \\
\hline Plane7 & 0.800 & 2.815 \\
\hline Plane8 & 0.900 & 4.815 \\
\hline Plane9 & 0.900 & 6.815 \\
\hline Plane10 & 1.000 & 12.815 \\
\hline
\end{tabular}




\subsubsection{Wind Tunnel Coordinate Reference System}

The wind tunnel is defined as a 3D Cartesian grid, where the origin sits on the lower left corner at the entrance facing the wind (Figure 4-9). In this way, $X$ represents the transverse coordinate, $\mathrm{Y}$ the vertical, and $\mathrm{Z}$ the longitudinal coordinate. With this configuration, the exhaust reference coordinates are $\mathrm{X}=3263 \mathrm{~mm}, \mathrm{Y}=3415 \mathrm{~mm}$, and $\mathrm{Z}=2845 \mathrm{~mm}$. All the points in the data file present the absolute coordinates.

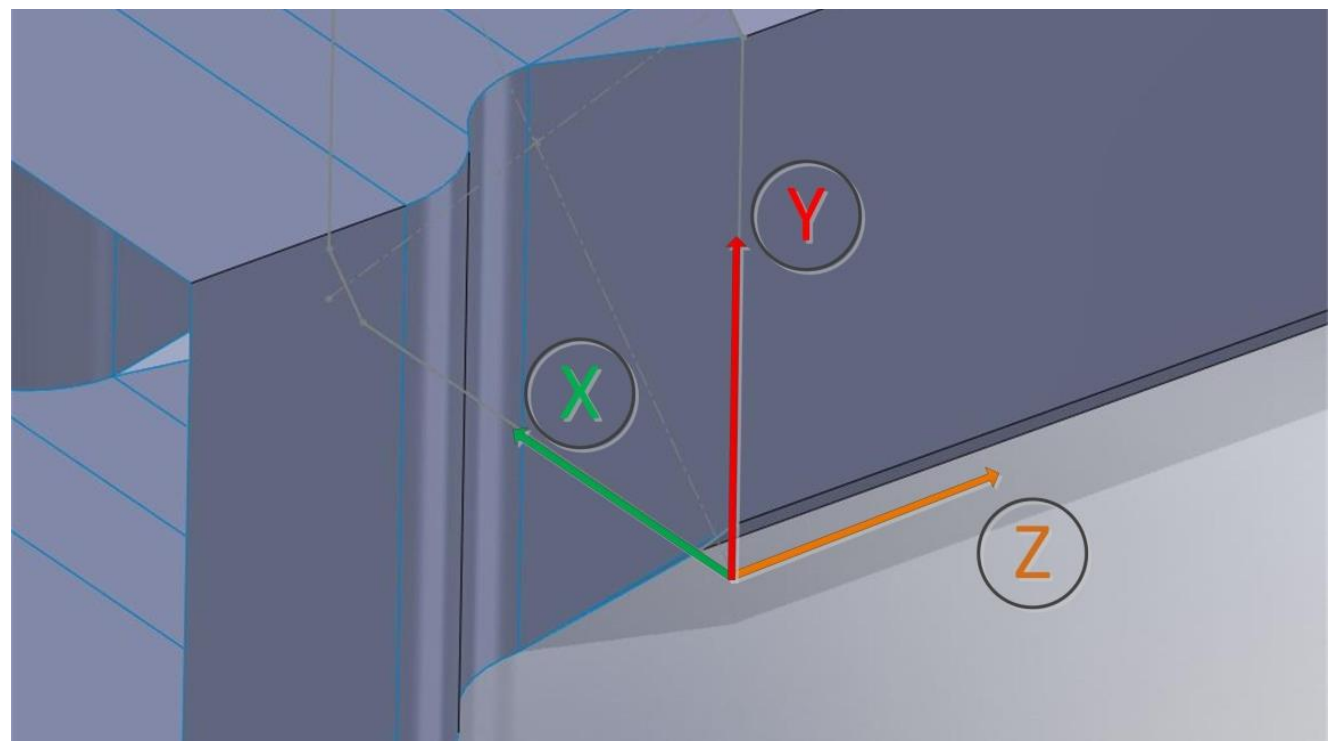

Figure 4-9: Wind Tunnel coordinate reference system

\subsubsection{Data File Dictionary}

The collected and untreated data are presented in an Excel worksheet (.xlsx), one file per each combination of truck and wind speed. In each Excel worksheet, each tab collects the channels recorded for all the points of a plane. The channels of each point are defined as a block, and the blocks are appended one under the other, as the points are sampled. Each block is composed of 68 channels, listed and described in Table 4-11. 
Table 4-11: Data File Dictionary

\begin{tabular}{|c|c|c|c|c|}
\hline & Channel Name & Unit of Measure & Description & $\begin{array}{l}\text { Sampling } \\
\text { Station }\end{array}$ \\
\hline 1 & $X$ & {$[\mathrm{~mm}]$} & $\mathrm{X}$ position of the probe & $\begin{array}{l}\text { Sampling } \\
\text { Cart }\end{array}$ \\
\hline 2 & $\mathrm{Y}$ & {$[\mathrm{mm}]$} & Y position of the probe & $\begin{array}{l}\text { Sampling } \\
\text { Cart }\end{array}$ \\
\hline 3 & $\mathrm{Z}$ & {$[\mathrm{mm}]$} & $\mathrm{Z}$ position of the probe & $\begin{array}{l}\text { Sampling } \\
\text { Cart }\end{array}$ \\
\hline 4 & CO21_probe & [ppm] & $\begin{array}{c}\mathrm{CO} 2 \text { low-range } \\
\text { Analyzer }\end{array}$ & $\begin{array}{l}\text { Sampling } \\
\text { Cart }\end{array}$ \\
\hline 5 & CO2m_probe & {$[\%]$} & $\begin{array}{c}\text { CO2 mid-range } \\
\text { Analyzer }\end{array}$ & $\begin{array}{l}\text { Sampling } \\
\text { Cart }\end{array}$ \\
\hline 6 & $\mathrm{CO} 2 \mathrm{~h} \_$probe & {$[\%]$} & $\begin{array}{c}\text { CO2 high-range } \\
\text { Analyzer }\end{array}$ & $\begin{array}{l}\text { Sampling } \\
\text { Cart }\end{array}$ \\
\hline 7 & CO2_probe & {$[\%]$} & $\begin{array}{l}\text { Proposed CO2 } \\
\text { combined signal }\end{array}$ & $\begin{array}{l}\text { Sampling } \\
\text { Cart }\end{array}$ \\
\hline 8 & NOx_probe & {$[\mathrm{ppm}]$} & NOx Analyzer & $\begin{array}{l}\text { Sampling } \\
\text { Cart }\end{array}$ \\
\hline 9 & tot_amine_probe & [ppm] & NOX-Amines Analyzer & $\begin{array}{l}\text { Sampling } \\
\text { Cart }\end{array}$ \\
\hline 10 & DustTrak & {$[\mathrm{mg} / \mathrm{m} 3]$} & DustTrak Analyzer & $\begin{array}{l}\text { Sampling } \\
\text { Cart }\end{array}$ \\
\hline 11 & aeth & [ng/m3] & $\begin{array}{c}\text { MicroAethalometer } \\
\text { Analyzer }\end{array}$ & $\begin{array}{l}\text { Sampling } \\
\text { Cart }\end{array}$ \\
\hline 12 & eeps & {$[\mathrm{dn} / \mathrm{d} \log (\mathrm{Dp})]$} & $\begin{array}{c}\text { Particle Concentration } \\
\text { @ } 6.04 \mathrm{~nm}\end{array}$ & $\begin{array}{l}\text { Sampling } \\
\text { Cart }\end{array}$ \\
\hline 13 & eeps & {$[\mathrm{dn} / \mathrm{d} \log (\mathrm{Dp})]$} & $\begin{array}{c}\text { Particle Concentration } \\
\text { @ 6.98nm }\end{array}$ & $\begin{array}{l}\text { Sampling } \\
\text { Cart }\end{array}$ \\
\hline 14 & eeps & {$[\mathrm{dn} / \mathrm{d} \log (\mathrm{Dp})]$} & $\begin{array}{c}\text { Particle Concentration } \\
\text { @ 8.06nm }\end{array}$ & $\begin{array}{l}\text { Sampling } \\
\text { Cart }\end{array}$ \\
\hline 15 & eeps & {$[\mathrm{dn} / \mathrm{d} \log (\mathrm{Dp})]$} & $\begin{array}{c}\text { Particle Concentration } \\
\text { @ } 9.31 \mathrm{~nm}\end{array}$ & $\begin{array}{l}\text { Sampling } \\
\text { Cart }\end{array}$ \\
\hline 16 & eeps & {$[\mathrm{dn} / \mathrm{d} \log (\mathrm{Dp})]$} & $\begin{array}{c}\text { Particle Concentration } \\
\text { @ } 10.8 \mathrm{~nm}\end{array}$ & $\begin{array}{l}\text { Sampling } \\
\text { Cart }\end{array}$ \\
\hline 17 & eeps & {$[\mathrm{dn} / \mathrm{d} \log (\mathrm{Dp})]$} & $\begin{array}{c}\text { Particle Concentration } \\
\text { @ } 12.4 \mathrm{~nm}\end{array}$ & $\begin{array}{l}\text { Sampling } \\
\text { Cart }\end{array}$ \\
\hline 18 & eeps & {$[\mathrm{dn} / \mathrm{d} \log (\mathrm{Dp})]$} & $\begin{array}{c}\text { Particle Concentration } \\
\text { @ } 14.3 \mathrm{~nm}\end{array}$ & $\begin{array}{l}\text { Sampling } \\
\text { Cart }\end{array}$ \\
\hline 19 & eeps & {$[\mathrm{dn} / \mathrm{d} \log (\mathrm{Dp})]$} & $\begin{array}{c}\text { Particle Concentration } \\
\text { @ } 16.5 \mathrm{~nm}\end{array}$ & $\begin{array}{l}\text { Sampling } \\
\text { Cart }\end{array}$ \\
\hline
\end{tabular}




\begin{tabular}{|c|c|c|c|c|}
\hline & eeps & {$[\mathrm{dn} / \mathrm{d} \log (\mathrm{Dp})]$} & $\begin{array}{c}\text { Particle Concentration } \\
\text { @ } 19.1 \mathrm{~nm}\end{array}$ & $\begin{array}{c}\text { Sampling } \\
\text { Cart }\end{array}$ \\
\hline 21 & eeps & {$[\mathrm{dn} / \mathrm{d} \log (\mathrm{Dp})]$} & $\begin{array}{c}\text { Particle Concentration } \\
\text { @ 22.1nm }\end{array}$ & $\begin{array}{c}\text { Sampling } \\
\text { Cart }\end{array}$ \\
\hline 22 & eeps & {$[\mathrm{dn} / \mathrm{d} \log (\mathrm{Dp})]$} & $\begin{array}{c}\text { Particle Concentration } \\
\text { @ 25.5nm }\end{array}$ & $\begin{array}{c}\text { Sampling } \\
\text { Cart }\end{array}$ \\
\hline 23 & eeps & {$[\mathrm{dn} / \mathrm{dlog}(\mathrm{Dp})]$} & $\begin{array}{c}\text { Particle Concentration } \\
\text { @ 29.4nm }\end{array}$ & $\begin{array}{c}\text { Sampling } \\
\text { Cart }\end{array}$ \\
\hline 24 & eeps & {$[\mathrm{dn} / \mathrm{dlog}(\mathrm{Dp})]$} & $\begin{array}{c}\text { Particle Concentration } \\
\text { @ } 34 \mathrm{~nm}\end{array}$ & $\begin{array}{c}\text { Sampling } \\
\text { Cart }\end{array}$ \\
\hline 25 & eeps & {$[\mathrm{dn} / \mathrm{d} \log (\mathrm{Dp})]$} & $\begin{array}{c}\text { Particle Concentration } \\
\text { @ 39.2nm }\end{array}$ & $\begin{array}{c}\text { Sampling } \\
\text { Cart }\end{array}$ \\
\hline 26 & eeps & {$[\mathrm{dn} / \mathrm{d} \log (\mathrm{Dp})]$} & $\begin{array}{c}\text { Particle Concentration } \\
\text { @ 45.3nm }\end{array}$ & $\begin{array}{c}\text { Sampling } \\
\text { Cart }\end{array}$ \\
\hline 27 & eeps & [dn/dlog(Dp)] & $\begin{array}{c}\text { Particle Concentration } \\
\text { @ 52.3nm }\end{array}$ & $\begin{array}{c}\text { Sampling } \\
\text { Cart }\end{array}$ \\
\hline 28 & eeps & {$[\mathrm{dn} / \mathrm{dlog}(\mathrm{Dp})]$} & $\begin{array}{c}\text { Particle Concentration } \\
\text { @ 60.4nm }\end{array}$ & $\begin{array}{c}\text { Sampling } \\
\text { Cart }\end{array}$ \\
\hline 29 & eeps & {$[\mathrm{dn} / \mathrm{d} \log (\mathrm{Dp})]$} & $\begin{array}{c}\text { Particle Concentration } \\
\text { @ 69.8nm }\end{array}$ & $\begin{array}{c}\text { Sampling } \\
\text { Cart }\end{array}$ \\
\hline 30 & eeps & {$[\mathrm{dn} / \mathrm{d} \log (\mathrm{Dp})]$} & $\begin{array}{c}\text { Particle Concentration } \\
\text { @ 80.6nm }\end{array}$ & $\begin{array}{c}\text { Sampling } \\
\text { Cart }\end{array}$ \\
\hline 31 & eeps & {$[\mathrm{dn} / \mathrm{d} \log (\mathrm{Dp})]$} & $\begin{array}{c}\text { Particle Concentration } \\
\text { @ 93.1nm }\end{array}$ & $\begin{array}{c}\text { Sampling } \\
\text { Cart }\end{array}$ \\
\hline 32 & eeps & {$[\mathrm{dn} / \mathrm{d} \log (\mathrm{Dp})]$} & $\begin{array}{l}\text { Particle Concentration } \\
\text { @ } 107.5 \mathrm{~nm}\end{array}$ & $\begin{array}{c}\text { Sampling } \\
\text { Cart }\end{array}$ \\
\hline 33 & eeps & {$[\mathrm{dn} / \mathrm{dlog}(\mathrm{Dp})]$} & $\begin{array}{c}\text { Particle Concentration } \\
\text { @ } 124.1 \mathrm{~nm}\end{array}$ & $\begin{array}{l}\text { Sampling } \\
\text { Cart }\end{array}$ \\
\hline 34 & eeps & {$[\mathrm{dn} / \mathrm{dlog}(\mathrm{Dp})]$} & $\begin{array}{l}\text { Particle Concentration } \\
\text { @ } 143.3 \mathrm{~nm}\end{array}$ & $\begin{array}{c}\text { Sampling } \\
\text { Cart }\end{array}$ \\
\hline 35 & eeps & {$[\mathrm{dn} / \mathrm{d} \log (\mathrm{Dp})]$} & $\begin{array}{c}\text { Particle Concentration } \\
\text { @ } 165.5 \mathrm{~nm}\end{array}$ & $\begin{array}{c}\text { Sampling } \\
\text { Cart }\end{array}$ \\
\hline 36 & eeps & {$[\mathrm{dn} / \mathrm{d} \log (\mathrm{Dp})]$} & $\begin{array}{l}\text { Particle Concentration } \\
\text { @ } 191.1 \mathrm{~nm}\end{array}$ & $\begin{array}{c}\text { Sampling } \\
\text { Cart }\end{array}$ \\
\hline 37 & eeps & {$[\mathrm{dn} / \mathrm{d} \log (\mathrm{Dp})]$} & $\begin{array}{l}\text { Particle Concentration } \\
\text { @ 220.7nm }\end{array}$ & $\begin{array}{c}\text { Sampling } \\
\text { Cart }\end{array}$ \\
\hline 38 & eeps & {$[\mathrm{dn} / \mathrm{d} \log (\mathrm{Dp})]$} & $\begin{array}{c}\text { Particle Concentration } \\
\text { @ 254.8nm }\end{array}$ & $\begin{array}{c}\text { Sampling } \\
\text { Cart }\end{array}$ \\
\hline 39 & eeps & {$[\mathrm{dn} / \mathrm{dlog}(\mathrm{Dp})]$} & $\begin{array}{c}\text { Particle Concentration } \\
\text { @ 294.3nm }\end{array}$ & $\begin{array}{c}\text { Sampling } \\
\text { Cart }\end{array}$ \\
\hline 40 & eeps & {$[\mathrm{dn} / \mathrm{dlog}(\mathrm{Dp})]$} & $\begin{array}{c}\text { Particle Concentration } \\
\text { @ 339.8nm }\end{array}$ & $\begin{array}{c}\text { Sampling } \\
\text { Cart }\end{array}$ \\
\hline 41 & eeps & {$[\mathrm{dn} / \mathrm{dlog}(\mathrm{Dp})]$} & $\begin{array}{c}\text { Particle Concentration } \\
\text { @ } 392.4 \mathrm{~nm}\end{array}$ & $\begin{array}{c}\text { Sampling } \\
\text { Cart }\end{array}$ \\
\hline
\end{tabular}




\begin{tabular}{|c|c|c|c|c|}
\hline & eeps & {$[\mathrm{dn} / \mathrm{d} \log (\mathrm{Dp})]$} & $\begin{array}{c}\text { Particle Concentration } \\
\text { @ 453.2nm }\end{array}$ & $\begin{array}{l}\text { Sampling } \\
\text { Cart }\end{array}$ \\
\hline 43 & eeps & [dn/dlog(Dp)] & $\begin{array}{c}\text { Particle Concentration } \\
\text { @ 523.3nm }\end{array}$ & $\begin{array}{l}\text { Sampling } \\
\text { Cart }\end{array}$ \\
\hline 44 & temp_probe & {$\left[{ }^{\circ} \mathrm{C}\right]$} & $\begin{array}{c}\text { Plume temperature @ } \\
\text { the probe }\end{array}$ & $\begin{array}{l}\text { Sampling } \\
\text { Cart }\end{array}$ \\
\hline 45 & flow_probe & {$[\mathrm{scfm}]$} & Flow rate @ the probe & $\begin{array}{c}\text { Sampling } \\
\text { Cart }\end{array}$ \\
\hline 46 & HWA_probe & {$[\mathrm{m} / \mathrm{s}]$} & Wind speed @ the probe & $\begin{array}{l}\text { Sampling } \\
\text { Cart }\end{array}$ \\
\hline 47 & $\mathrm{CO} 2$ _raw & {$[\%]$} & CO2 Analyzer & TREL \\
\hline 48 & CO_raw & [ppm] & CO Analyzer & TREL \\
\hline 49 & NOx_raw & [ppm] & NOx Analyzer & TREL \\
\hline 50 & HC_raw & [ppm] & THC Analyzer & TREL \\
\hline 51 & AVL_MSS_raw & {$[\mathrm{mg} / \mathrm{m} 3]$} & AVL MSS Analyzer & TREL \\
\hline 52 & ex_flow_raw & {$[\mathrm{scfm}]$} & Exhaust flow rate & TREL \\
\hline 53 & ex_annu_temp_raw & {$\left[{ }^{\circ} \mathrm{C}\right]$} & $\begin{array}{l}\text { Exhaust temperature } \\
\text { @ raw sampling point }\end{array}$ & TREL \\
\hline 54 & ex_stack_temp_raw & {$\left[{ }^{\circ} \mathrm{F}\right]$} & $\begin{array}{c}\text { Exhaust temperature } \\
\text { @ stack exit }\end{array}$ & TREL \\
\hline 55 & dyno_speed & [rpm] & $\begin{array}{c}\text { Chassis Dynamometer } \\
\text { speed }\end{array}$ & TREL \\
\hline 56 & dyno_torque & [ft-lb] & $\begin{array}{l}\text { Chassis Dynamometer } \\
\text { absorbed torque }\end{array}$ & TREL \\
\hline 57 & dyno_power & {$[\mathrm{Hp}]$} & $\begin{array}{l}\text { Chassis Dynamometer } \\
\text { absorbed power }\end{array}$ & TREL \\
\hline 58 & vehicle_speed & {$[\mathrm{mph}]$} & Vehicle speed & TREL \\
\hline 59 & fuel_mass_raw & {$[\mathrm{lb}]$} & $\begin{array}{c}\text { Fuel scale } \\
\text { measurements }\end{array}$ & TREL \\
\hline 60 & fuel_temp_raw & {$\left[{ }^{\circ} \mathrm{C}\right]$} & Fuel temperature & TREL \\
\hline 61 & HWA_wt & {$[\mathrm{m} / \mathrm{s}]$} & $\begin{array}{l}\text { Wind Tunnel wind } \\
\text { speed }\end{array}$ & $\begin{array}{l}\text { Background } \\
\text { Air }\end{array}$ \\
\hline 62 & temp_inlet & {$\left[{ }^{\circ} \mathrm{F}\right]$} & $\begin{array}{l}\text { Background Air inlet } \\
\text { temperature }\end{array}$ & $\begin{array}{c}\text { Background } \\
\text { Air }\end{array}$ \\
\hline 63 & dewpoint_inlet & {$\left[{ }^{\circ} \mathrm{C}\right]$} & $\begin{array}{l}\text { Background Air inlet } \\
\text { dew point temperature }\end{array}$ & $\begin{array}{l}\text { Background } \\
\text { Air }\end{array}$ \\
\hline
\end{tabular}




\begin{tabular}{|c|c|c|c|c|}
\hline 64 & temp_weather & {$\left[{ }^{\circ} \mathrm{C}\right]$} & $\begin{array}{l}\text { local temperature from } \\
\text { weather station }\end{array}$ & $\begin{array}{c}\text { Background } \\
\text { Air }\end{array}$ \\
\hline 65 & dewpoint_weather & {$\left[{ }^{\circ} \mathrm{C}\right]$} & $\begin{array}{c}\text { local dew point } \\
\text { temperature } \\
\text { from weather station }\end{array}$ & $\begin{array}{c}\text { Background } \\
\text { Air }\end{array}$ \\
\hline 66 & wind_int_weather & [mph] & $\begin{array}{l}\text { local wind from weather } \\
\text { station }\end{array}$ & $\begin{array}{c}\text { Background } \\
\text { Air }\end{array}$ \\
\hline 67 & wind_dir_weather & {$\left[{ }^{\circ}\right]$} & $\begin{array}{l}\text { local wind from weather } \\
\text { station }\end{array}$ & $\begin{array}{c}\text { Background } \\
\text { Air }\end{array}$ \\
\hline 68 & barometer_weather & {$[\mathrm{Pa}]$} & $\begin{array}{c}\text { local barometric } \\
\text { pressure } \\
\text { from weather station }\end{array}$ & $\begin{array}{c}\text { Background } \\
\text { Air }\end{array}$ \\
\hline
\end{tabular}

In summary, each vehicle will present three Excel sheets, comprehensive of the three tests, plus a fourth which contains the PM size particle and distributions from the raw exhaust.

\subsubsection{WVU QA/QC Assurance Plan}

Quality control and quality assurance procedures adopted by the WVU wind tunnel and the WVU Transportable Heavy-Duty Vehicle Emissions Laboratory are given below:

\section{Emissions Testing}

The laboratories are capable of measuring regulated and non-regulated engine or vehicle emissions such as $\mathrm{CO}, \mathrm{NO}_{\mathrm{x}}$, THC, TPM, and $\mathrm{CO}_{2}$. Reliable sampling is assured through system design, periodic system inspection, and scheduled instrument calibration. 


\section{$\underline{\text { Sampling Lines and Probes }}$}

The sampling streams use separate sampling probes and lines with their own pumps (heated in the case of NOx, THC, and also $\mathrm{CO} / \mathrm{CO}_{2}$ to avoid condensation of moisture in the lines). This design feature ensures reliable operation of the $\mathrm{THC}$ and $\mathrm{NO}_{\mathrm{x}}$ analyzers.

Great care is taken in verifying that emissions measurement equipment is in order before commencement of emissions testing. Prior to the performance of a test schedule, supervisory personnel verify that all sample probes in the raw exhaust are properly placed and that the integrity of the sampling systems has been maintained. The check consists of all the probes to be equidistant from the center of the tunnel and oriented with their sample inlets facing upstream. The sampling lines are leak checked (by pressurization) and backflushed with high pressure air in order to clean the lines of residual particulate matter. Heated sampling lines and their associated control systems (PID temperature controllers and associated thermocouples) are checked to ensure continuity between the controller, heater elements, and thermocouples. The temperature settings are also verified. THC sampling probes and lines are maintained at $375^{\circ} \mathrm{F}$ while $\mathrm{NO}_{\mathrm{x}}$ lines and probes are maintained at $250^{\circ}$ F. Sample line temperatures, which are monitored by the operator, are displayed on the sampling cabinet in various locations.

\section{$\underline{\text { Thermocouples and Pressure transducers }}$}

Thermocouples type $\mathrm{K}$ are used for temperature measurements and calibrated by a certified FLUKE model 714 thermocouples calibrator from 0 to $100 \%$ of their range in $10 \%$ increments. The thermocouple readings are allowed to stabilize at each measurement 
point and a computer averaged (100 points) reading of the instrument is recorded. The eleven data points and their corresponding temperature are recorded and a fifth-degree polynomial is fitted to the data to complete the calibration data file. Pressure transducers are calibrated with a certified pressure analyzer from 0 to $100 \%$ of their range in $10 \%$ increments. The pressure readings are allowed to stabilize at each measurement point and a computer averaged (100 points) reading of the instrument is recorded. The eleven data points and their corresponding temperature are recorded and a linear polynomial is fitted to the data to complete the calibration data file.

\section{Exhaust Transfer Tube}

The exhaust transfer tube, which routes exhaust from the engine exhaust system to the wind tunnel, is checked for leaks after assembly. Joints in the tube are sealed using a high-temperature aluminum tape and periodically checked to ensure integrity. The exhaust transfer tube is insulated to minimize errors in PM measurement due to thermophoretic effects.

\section{Calibration and Calibration Gases}

The gases used to calibrate the emissions measurement instruments are certified by the supplier to have an accuracy of $1 \%$ traceable to NIST. The laboratory uses the same gases for calibration, span, and zero gases. All span and calibration gases have concentrations equal to approximately $85 \%$ to $95 \%$ of full-scale. All emissions measurement instruments are calibrated using appropriate ranges of calibration gases for 
the engine or vehicle being tested. These calibrations are performed before each series of tests and after any instrument maintenance has been performed. $\mathrm{HC}, \mathrm{CO}, \mathrm{NO}_{\mathrm{x}}$, and $\mathrm{CO}_{2}$ instruments are calibrated by sampling a varying mixture of zero air and span gas (calibration gas) through a gas divider from 0 to $100 \%$ of their range in $10 \%$ increments. The instrument readings are allowed to stabilize at each measurement point and a computer averaged (100 points) reading of the instrument is recorded. The eleven data points and their corresponding gas concentrations are recorded and a second-degree (third-degree in case of NDIRs) polynomial is fitted to the data to complete the calibration data file. The calibration file overwrites the previous calibration file on the main computer (each test data disk contains the calibration files from that test) to prevent using incorrect calibration files for testing. No gas cylinder is used if the pressure drops below 200 psig.

\section{$\underline{\text { Additional Calibration and Maintenance Procedures }}$}

In addition to the calibration procedures, each analyzer is subjected to a range of checks and maintenance procedures as described below.

\section{$\underline{\text { Hydrocarbon Analyzer }}$}

The THC analyzer is subjected to the 'FID burner peaking process' to get the highest flame ionization detector (FID) response. This process involves measuring and recording the response of the instrument to $100 \%$ span gas and zero air with various settings of FID burner fuel and air. Upon completion of the FID burner peaking process, the fuel and air settings of the FID are placed at the setting which produced the highest instrument 
response, and the analyzer is calibrated. A HC hang-up check, following the procedures outlined in 40 CFR, Part 1065, is also performed on the heated FID. If the differences in the responses are more than two percent, the sampling probe is back-flushed via direct injection of zero air into the analyzer and through the 'overflow' sampling probe, and steps are taken to rectify the problem.

\section{$\underline{\mathrm{NO}_{\mathrm{x}} \text { Analyzers }}$}

On a monthly basis, a $\mathrm{NO}_{\mathrm{x}}$ efficiency test is performed on the NOx analyzers. This test is performed to ensure that the analyzer converter (which converts $\mathrm{NO}_{2}$ to $\mathrm{NO}$ ) is performing satisfactorily. A conversion efficiency of less than $90 \%$ is considered a failure and maintenance is performed to rectify the situation. Filters in the $\mathrm{NO}_{\mathrm{x}}$ sampling are visually inspected and replaced after analyzer maintenance has been performed and whenever necessary.

\section{$\underline{\mathrm{CO} / \mathrm{CO}_{2}} \underline{\text { Analyzers }}$}

Since moisture can affect the operation of the NDIR analyzers used for CO and $\mathrm{CO}_{2}$, a water interference check is performed. The sample flow is passed through a refrigerator dryer to lower the dew point of the sample stream before it reaches the NDIR.

\section{Particulate Matter Sampling}

PM sampling is obtained with three different spectrometers (SMPS ${ }^{\mathrm{TM}}$, EEPS ${ }^{\mathrm{TM}}$, DMS) and three soot analyzers (DustTrak ${ }^{\mathrm{TM}}$, MicroAethalometer and MSS). All 
instruments are operated, cleaned, and zeroed according to their respective manuals and common procedures, applied as extension of CFR 1065 indications on quality measurements.

Data Analysis and Reporting

Quality assurance for data reported from testing is achieved using multiple checks.

First, the operators of the laboratory, including the senior supervisory personnel as well as technicians, have a vast amount of experience in testing a multitude of different vehicle/engine/fuel combinations. This experience is valuable in quality assurance since it allows the laboratory operators to identify problems at the test site. As a test is performed, data is logged to a local hard disk on the data acquisition computer. After the test is completed, data is backup on two separated drives. A data reduction program is used to extract emissions data from a binary data file and translate it into the appropriate units using calibration files. On-site examination of the data is done by supervisory personnel to determine the accuracy of the data in order that equipment malfunctions can be identified and promptly corrected.

\subsection{Class-8 Heavy-duty Diesel Vehicles}

The vehicles chosen for this test campaign are meant to represent today's on-road legacy fleet. Three vehicles compliant with different on-road emissions standards and with different exhaust aftertreatment configurations allow for the accurate depiction of the contribution of each to the overall PM emissions. The model years (MY) are 1995, 2008, and 2011; the following Table 4-12 helps to show the differences in gaseous and PM emissions standards. 
Table 4-12: US-EPA Emissions Standards for On-Road Heavy Duty Diesel Engines (HDDE) regarding the tested vehicles, the emissions are indicated in gr/bhp-hr.

\begin{tabular}{|l|c|c|c|c|c|c|}
\hline Model Year & HC & CO & NOx $_{\mathbf{x}}$ & NMHC + NOx & NMHC & PM \\
\hline $\mathbf{1 9 9 5}$ & 1.3 & 15.5 & 5.0 & & - & 0.10 \\
\hline $\mathbf{2 0 0 4}$ and later (opt.1) & - & 15.5 & - & 2.4 & - & 0.10 \\
\hline $\mathbf{2 0 0 4}$ and later (opt.2) & - & 15.5 & - & 2.5 & 0.5 & 0.10 \\
\hline $\mathbf{2 0 0 7}$ and later (phase in) & - & 15.5 & 0.2 & - & 0.14 & 0.01 \\
\hline $\mathbf{2 0 1 0}$ and later & - & 15.5 & 0.2 & - & 0.14 & 0.01 \\
\hline
\end{tabular}

The following emission standards apply to new diesel engines used in heavy-duty highway vehicles (HDDVs). The current federal definition of a compression-ignition (diesel) engine is based on the engine cycle, rather than the ignition mechanism, with the presence of a throttle as an indicator to distinguish between diesel-cycle and Otto-cycle operation. Regulating power by controlling the fuel supply in lieu of a throttle corresponds with lean combustion and the diesel-cycle operation. In this way, a natural gas-fueled engine equipped with a sparkplug is considered a compression-ignition engine. HDDVs are defined as vehicles of gross vehicle weight rating (GVWR) of above 8,500 lbs. in federal jurisdiction and above 14,000 lbs. in California (model year 1995 and later). 
Diesel engines used in heavy-duty vehicles are further divided into service classes by GVWR, as follows:

- Light heavy-duty diesel engines: 8,500 $<$ LHDDE $<19,500$

$$
(14,000<\text { LHDDE }<19,500 \text { in California, 1995+) }
$$

- Medium heavy-duty diesel engines: $19,500 \leq$ MHDDE $\leq 33,000$

- Heavy heavy-duty diesel engines (including urban buses): HHDDE $>33,000$

Applicable to the 1994 and following year standards, sulfur content in the certification fuel has been reduced to $500 \mathrm{ppm}$ wt. Compliance with emission standards has to be demonstrated over the useful life of the engine, which was adopted as follows (federal and California):

- LHDDE-8 years/110,000 miles (whichever occurs first)

- MHDDE— 8 years/185,000 miles

- HHDDE-8 years/290,000 miles

In October 1997, the EPA created new emission standards for heavy-duty diesel truck and bus engines for upcoming models following the 2004 model year. These standards reflect the provisions of the Statement of Principles (SOP) signed in 1995 by the EPA, CARB, and the manufacturers of heavy-duty diesel engines. The goal was to reduce $\mathrm{NO}_{\mathrm{x}}$ emissions from HDDEs to levels approximately $2.0 \mathrm{~g} / \mathrm{bhp}$-hr beginning in 2004. Manufacturers had the flexibility to certify their engines to one of the two options. 
The EPA established revised useful engine lives, with extended requirements for the heavy HDDE service class, as follows:

- LHDDE-110,000 miles/10 years

- MHDDE—185,000 miles/10 years

- HHDDE-435,000 miles/10 years/22,000 hours

The emission warranty remained at 5 years/100,000 miles.

With the exception of turbocharged and supercharged diesel fueled engines, discharge of crankcase emissions was not allowed for any new 2004 or later model year engines. The 2004 federal standards for highway trucks were harmonized with California standards, with the intent that manufacturers could use a single engine or machine design for both markets. California certifications for model years 2005-2007, however, additionally require SET testing, and NTE limits of $1.25 \times$ FTP standards.

On December 21, 2000 the EPA signed emission standards for model years 2007 and later HDDEs. The rule included two components: emission standards and diesel fuel regulations.

The PM emission standard took full effect in the 2007 heavy-duty engine model year. The $\mathrm{NO}_{\mathrm{x}}$ and NMHC standards were phased in for diesel engines between 2007 and 2010. The phase-in would be on a percent-of-sales basis: 50\% from 2007 to 2009 and $100 \%$ in 2010. Very few engines meeting the $0.20 \mathrm{~g} / \mathrm{bhp}-\mathrm{hr}$ NOx requirement actually appeared before 2010. In 2007, most manufacturers opted instead to meet a Family Emission Limit (FEL) around 1.2-1.5 g/bhp-hr NOx for most of their engines with a few manufacturers still certifying some of their engines as high as $2.5 \mathrm{~g} / \mathrm{bhp}-\mathrm{hr} \mathrm{NO}+\mathrm{NMHC}$. 
In addition to transient FTP testing, emission certification requirements also include:

- $\quad$ SET test, with limits equal to the FTP standards, and

- NTE testing with limits of $1.5 \times$ FTP standards for engines meeting a NOx FEL of $1.5 \mathrm{~g} / \mathrm{bhp}$-hr or less and $1.25 \times$ FTP standards for engines with a NOx FEL higher than $1.5 \mathrm{~g} / \mathrm{bhp}-\mathrm{hr}$.

Effective for the 2007 model year, the regulation maintained the earlier crankcase emission control exception for turbocharged HDDEs, but required that if they are emitted to the atmosphere, they be added to the exhaust emissions during testing. In this case, the deterioration of crankcase emissions must also be accounted for in exhaust deterioration factors.

Diesel fuel regulation limited the sulfur content in on-highway diesel fuel to 15 ppm wt., down from the previous $500 \mathrm{ppm}$. Refiners were required to start producing the 15 ppm sulfur fuel beginning June 1, 2006. At the terminal level, highway diesel fuel sold as low sulfur fuel met the 15 ppm sulfur standard as of July 15, 2006. For retail stations and wholesale purchasers, highway diesel fuel sold as low sulfur fuel must meet the 15 ppm sulfur standard by September 1, 2006. 


\subsubsection{Pre-2007}

The Pre-2007 vehicle is not equipped with any aftertreatment devices. The vehicle is part of the CAFEE fleet, and additional information is summarized in Table 4-13 and Table 4-14.

Table 4-13: Pre-2007 vehicle specifications

\begin{tabular}{lc}
\hline Parameter & Value \\
\hline Chassis Manufacturer / Model & Mack Trucks Inc / CH613 \\
VIN & 1M1AA13Y95WO54437 \\
Class & 8 \\
Vehicle Model Year (MY) & 1995 \\
Aftertreatment System & - \\
Fuel & Standard ULSD (<15ppm) \\
Emission Family & SMK728EJDARA (45) \\
Curb Weight [lbs.] & 15’000 \\
\hline
\end{tabular}

Table 4-14: Pre-2007 engine specification (*certification values)

\begin{tabular}{lc}
\hline Parameter & Value \\
\hline Engine Manufacturer & Mack Trucks Inc. \\
Engine Model & E7-400 \\
Engine Model Year & 1995 \\
Displacement [L] & 11.93 \\
Configuration / \# of Cylinders & In-line / 6 cylinder \\
Rated Power [Hp] & 400 @ 1800rpm \\
NOx [g/bhp-hr] & $4.5^{*}$ \\
PM [g/bhp-hr] & $0.09^{*}$ \\
\hline
\end{tabular}




\subsubsection{Post-2007}

The Post-2007 vehicle is equipped with DOC/DPF to meet the 2007 US EPA regulations. The vehicle was rented from FYDA Freightliner Inc. in Canonsburg, PA. Additional information is summarized in the following Table 4-15 and Table 4-16.

Table 4-15: Post-2007 vehicle specifications

\begin{tabular}{lc}
\hline Parameter & Value \\
\hline Chassis Manufacturer / Model & Freightliner C1120 \\
VIN & 1FUJA6CK89DAE7405 \\
Class & 8 \\
Vehicle Model Year (MY) & $08 / 2008$ \\
Aftertreatment System & DOC/DPF \\
Fuel & Standard ULSD $(<15 \mathrm{ppm})$ \\
Emission Family & 8DDXH14.0ELC \\
Curb Weight [lbs.] & 23,587 \\
\hline
\end{tabular}

Table 4-16: Post-2007 engine specification (*certification values)

\begin{tabular}{lc}
\hline Parameter & Value \\
\hline Engine Manufacturer & Detroit Diesel \\
Engine Model & SERIES 60 \\
Engine Model Year & S/N 06R 1012772 \\
Displacement [L] & 2008 \\
Configuration /\# of Cylinders & $14.0 \mathrm{~L}$ \\
Rated Power [Hp] & In-line / 6 cylinder \\
NOx [g/bhp-hr] & 515 @ 1800 \\
PM [g/bhp-hr] & $1.07^{*}$ \\
\hline
\end{tabular}




\subsubsection{Post-2010}

The Post-2010 vehicle is equipped with urea-based SCR and DOC/DPF assembly to meet the 2010 US EPA regulations. The vehicle was rented from Worldwide Equipment Inc. in Prestonsburg, KY. Additional information is summarized in Table 4-17 and Table 4-18.

Table 4-17: Post-2010 vehicle specifications

\begin{tabular}{lc}
\hline Parameter & Value \\
\hline Chassis Manufacturer / Model & Mack Trucks Inc. / CXU613 \\
VIN & 1M1AW07Y1CM017126 \\
Class & 8 \\
Vehicle Model Year (MY) & 2011 \\
Aftertreatment System & DOC / DPF / urea-SCR \\
Fuel & Standard ULSD (<15ppm) \\
Emission Family & BVPTH12.8S01 \\
Curb Weight [lbs.] & 15 '000 \\
\hline
\end{tabular}

Table 4-18: Post-2010 engine specifications (*certification values)

\begin{tabular}{lc}
\hline Parameter & Value \\
\hline Engine Manufacturer & Mack Trucks Inc. \\
Engine Model & MP8-445C \\
Engine Model Year & 2011 \\
Displacement [L] & 12.8 \\
Configuration / \# of Cylinders & In-line / 6 cylinder \\
Rated Power [Hp] & 445 @ 1500rpm \\
NOx [g/bhp-hr] & $0.12^{*}$ \\
PM [g/bhp-hr] & $0.003^{*}$ \\
\hline
\end{tabular}




\subsection{Test MATRIX}

In this test campaign, three different aftertreatment technologies were chosen, thus three different vehicles are going to be investigated. The first one reflects pre-2007 US EPA aftertreatment technology, one compliant with 2007 US EPA regulation and finally a 2010 US EPA compliant vehicle. Those three vehicles and their aftertreatment technologies represent their legacy fleet. Three different wind speed/road loads are chosen for this test campaign. The first one called Idle Test, is the typical configuration in which a truck is in a rest area with the engine in idling conditions with low wind (5mph). The second is the 20mph Test, where it is meant to simulate a truck cruising on a flat road in calm wind. According to the road load equation a high roof cabin at 20mph generates $40 \mathrm{hp}$ (see Equation 4-1) (Martyr \& Plint, 1995). The third and final test, the 35mph Test, simulates a truck cruising on a flat road in calm wind. According to the road load equation a high roof cabin at $35 \mathrm{mph}$ generates $63 \mathrm{hp}$.

$$
\begin{aligned}
& F=a_{0}+a_{1} V+a_{2} V^{2}+M \frac{d v}{d t}+M g \sin \vartheta \\
& a_{0}=\text { Component of rolling resistance } \\
& a_{1}=\text { Speed dependant resistance } \\
& M=\text { Mass of the Vehicle } \\
& V=\text { Vehicle Speed } \\
& \vartheta=\text { Road Slope }
\end{aligned}
$$


Table 4-19: Test Campaign Matrix

\begin{tabular}{lccc}
\hline & Idle Test & 20mph Test & 35mph Test \\
\hline Pre-2007 & 1 & 1 & 1 \\
Post-2007 & 1 & 1 & 1 \\
Post-2010 & 1 & 1 & 1 \\
\hline
\end{tabular}

In order to avoid boundary condition changes related to the weather(e.g. humidity and temperature), each combination of truck and wind/vehicle speed are tested in a single session for a time frame that does not exceed six hours, leading to a test matrix composed of nine combinations (see Table 4-19). Recalling the information indicated in the previous paragraph, the plume investigation was to be conducted using 130 sampling points divided into ten planes. Each plane presents the same sampling pattern, with different vertical dimensions and positions, to better focusing on the plume shape and dimension evolution. This configuration was selected after a preliminary study on plume evolution in order to describe its evolution with a limited number of points, an additional point p0 (point 0) was added to the original 130 sampling points. Point p0 is located right at the exhaust stack. The first 65 points and p0 present 100s sampling time (1000 data points), and the remaining points 50s (500 data points). This solution allows for the investigation of turbulence in areas close to the exhaust stack where the highly turbulent kinetic energy of the flow represents the predominant driver for the plume mixing and evolution. Far away from the exhaust stack, turbulent mixing loses most of its energy, and it is replaced by a slower gas diffusion process. Prior to any sampling, 30s of non-sampling stabilization time was 
observed once the probe reaches the desired position, accounting for the inertia of the measurement system. This configuration agrees with the limit of six hours per each combination of truck and wind speed. 


\section{Chapter 5: Characterization of Differences in Concentrations and Size Distributions of PM Emissions from Three US-EPA Regulation Compliant HDDTs at Different Wind and Vehicle Speeds}

\subsection{Performed Test MAtrix}

This task represents the core of the research with the performing of the test matrix defined in the previous chapter. The testing of the three vehicles spans from September 2012 to November 2012. Over the three-month period, a slight decrease in background temperature and humidity was observed from day-to-day although, no variations were observed within each test. Tests were performed only in calm wind (local wind speed magnitude below 5mph) and days without precipitation.

During the testing of the Post-2007 vehicle, PM emissions resulted higher than expected. After the complete analysis of the gathered data, it was determined that the DPF from the Post-2007 had partially failed (cracked), although it was still able to collect sufficient amounts of PM necessary to trigger regeneration activities. Even though, no visible inspection was performed on the DPF, a damage in it is the only way to have the soot level measured in the exhaust.

\subsection{BACKGROUND PM AIR AND RAW EMISSIONS}

The size of the WVU wind tunnel and the topography of the surrounding area helped to prevent the flow exiting the wind tunnel from re-entering the inlet. The wind tunnel is placed on a flat area with no building in the surroundings that could deviated the 
exiting flow to re-enter. The construction site is placed one mile away from any major roads or industrial plants and is assumed to attenuate variations of the background conditions over the test period as well as prevent increased PM concentrations due to either passing vehicles or industrial plant operations. Little to none traffic (less than 5 vehicle pass per day) was observed by local farm equipment during the tests, but no evidence indicated the effect on those vehicles on the measurements. These combined features led to guaranteeing an almost constant background PM over the testing. Figure 5- shows the PM particle size distribution and concentration for background air entering the wind tunnel. The measurements are gathered with the EEPS for 100s. The error bars for each particle size distribution are achieved as standard deviation from the time averaged value. Three different vehicle and wind speed measurements indicate almost constant PM is present, validating the assumptions on the position of the wind tunnel with respect to roads and industrial plant operations. Constant PM background is a necessary boundary condition to compare plume samples from different trucks. In addition, constant PM background reduces the uncertainty to which the exhaust and background PM interaction takes place. It is important to remark that the PM measured in the background air of the wind tunnel was found to be relatively low, pushing the EEPS to work at its lower measurable threshold causing higher noise and standard deviations to occur for larger particles. 


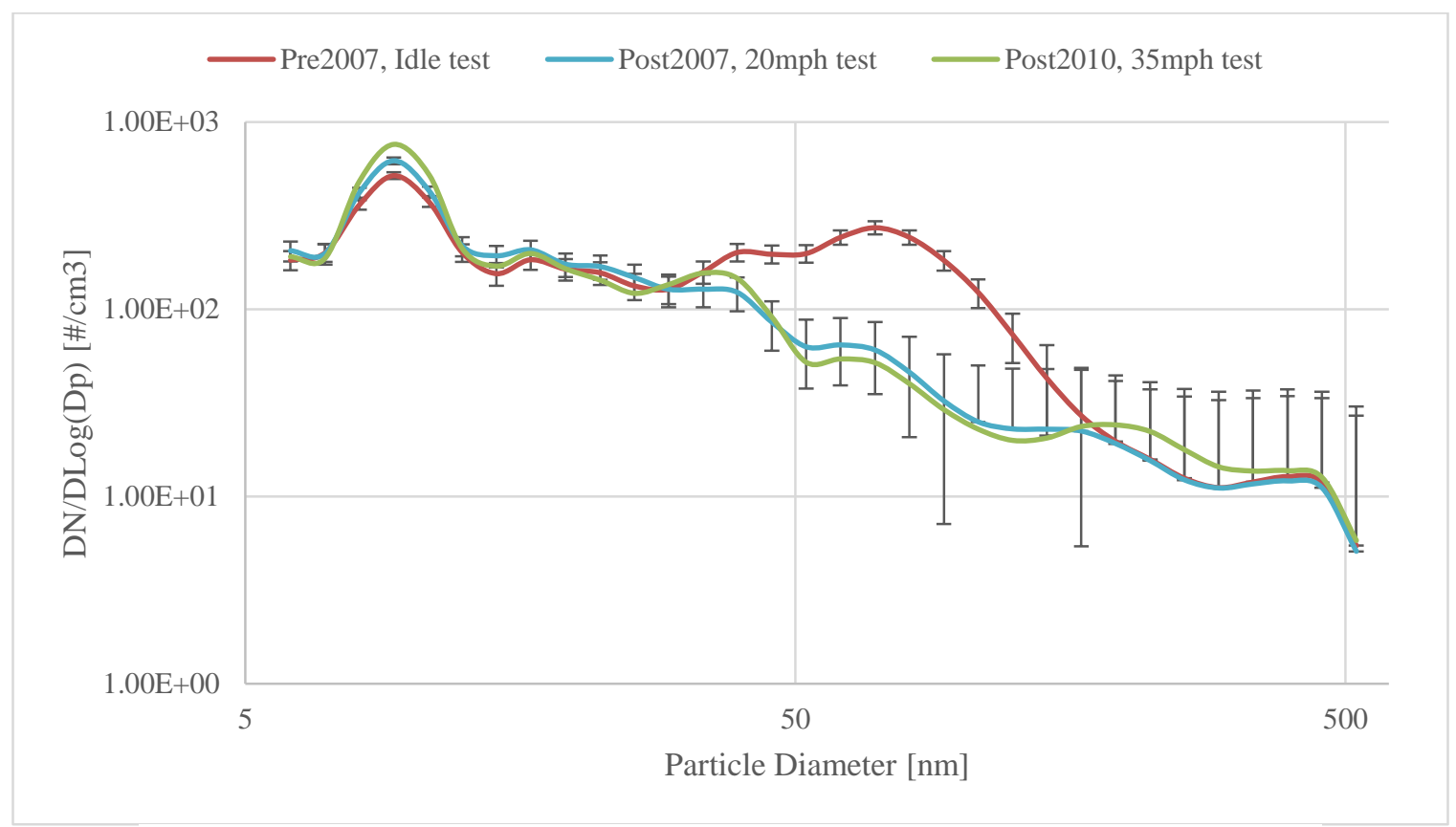

EEPS, Model 3090 Concentration Range Min Values are Typical RMS Noise with Filtered Inlet Air

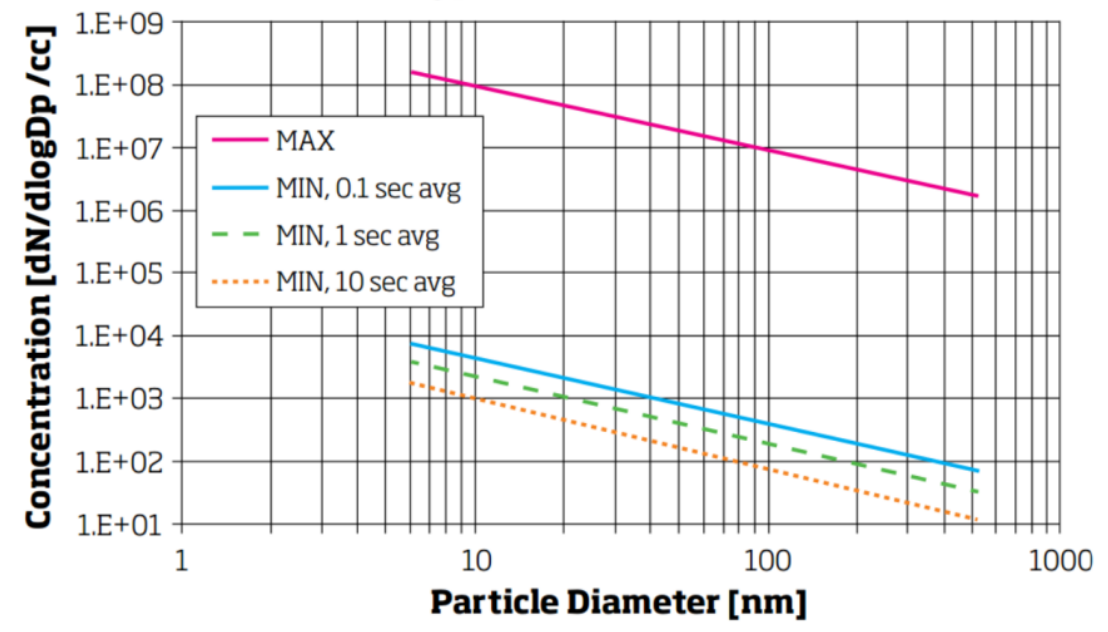

Figure 5-1: PM particle size distribution and concentration for the background air entering the wind tunnel during three different vehicle and wind speed tests and applicable range from TSI EEPS manual. Results indicate almost constant PM background air. Threshold Diagram is from TSI EEPS manual 
Gaseous components were also found to be constant for the entire duration of the testing campaign. $\mathrm{CO}_{2}$ and $\mathrm{NO}_{\mathrm{x}}$ emissions measurements fall within the prescribed values indicated by literature. In particular, $\mathrm{NO}_{\mathrm{x}}$ emission values fell within the accuracy and noise level of the instrument of 1ppm (Ardanese, et al., 2009) (Kittelson, Ayala, \& Olson, 2002) (Littera, et al., 2011).

Table 5-1: Background Air $\mathrm{CO}_{2}$ and $\mathrm{NO}_{\mathrm{x}}$ Emissions Concentration Averaged Over the Nine Tests.

\section{Emissions}

$\mathrm{CO}_{2}[\%]$

$\mathrm{NO}_{\mathbf{x}}[\mathrm{ppm}]$
$0.0450 \pm 0.0025$

$1 \pm 1$

Raw tailpipe emissions of the three test vehicles are presented on a concentration basis in. The presented data are time-averaged over the entire test length (approximately 6 hours long); $\mathrm{NO}_{\mathrm{x}}, \mathrm{CO}, \mathrm{CO}_{2}, \mathrm{HC}$ and soot mass estimate (AVL MSS) were continuously monitored during the test. The value of raw exhaust concentration emissions for each vehicle falls within the literature values of their respective families of regulation (Kittlelson, 1998) (Ardanese, et al., 2009) (Littera, et al., 2011).

Table 5-2, Table 5-3, and Table 5-4 show considerable differences of $\mathrm{CO}_{2}$ concentrations collected from the three vehicles. This is attributed to different engine efficiency points for the same power output. The engines have different displacement and overall setup, thus different engine calibrations. 
Table 5-2: Raw Tailpipe Gaseous and PM Soot Emissions for the Three Tested Vehicles Measured during the Idle Test

\begin{tabular}{lccc}
\hline Emissions & Pre-2007 & Post-2007 & Post-2010 \\
\hline CO2 [\%] & 1.346 & 3.287 & 2.622 \\
\hline CO [ppm] & 84 & 218 & 228 \\
\hline NOx [ppm] & 213 & 243 & 125 \\
\hline THC [ppm] & 110 & 63 & 27 \\
\hline AVL MSS [mg/m^3] & BDL & BDL & BDL \\
\hline Exhaust Flow Rate [scfm] & 119.48 & 77.20 & 82.97 \\
\hline Exhaust Temperature $\left[{ }^{\circ} \mathbf{C}\right]$ & 78.12 & 62.76 & 70.56 \\
\hline
\end{tabular}

Table 5-3: Raw Tailpipe Gaseous and PM Soot Emissions for the Three Tested Vehicles Measured During the 20mph Test

\begin{tabular}{lccc}
\hline Emissions & Pre-2007 & Post-2007 & Post-2010 \\
\hline CO2 [\%] & 4.434 & 8.376 & 5.625 \\
\hline CO [ppm] & 59 & BDL & BDL \\
\hline NOx $[\mathbf{p p m}]$ & 892 & 57 & 126 \\
\hline THC [ppm] & 49 & 8 & 1 \\
\hline AVL MSS [mg/m^3] & 0.529 & 0.260 & BDL \\
\hline Exhaust Flow Rate [scfm] & 299.74 & 246.10 & 292.41 \\
\hline Exhaust Temperature $\left[{ }^{\circ} \mathrm{C}\right]$ & 245.67 & 254.86 & 194.85 \\
\hline
\end{tabular}


Table 5-4: Raw Tailpipe Gaseous and PM Soot Emissions for the Three Tested Vehicles Measured During the 35mph Test

\begin{tabular}{lccc|}
\hline Emissions & Pre-2007 & Post-2007 & Post-2010 \\
\hline $\mathbf{C O}_{2}[\%$ \%] & 6.213 & 9.812 & 7.720 \\
\hline $\mathbf{C O}[\mathbf{p p m}]$ & 37 & $\mathrm{BDL}$ & $\mathrm{BDL}$ \\
\hline NOx $[\mathbf{p p m}]$ & 1004 & 243 & 1 \\
\hline THC [ppm] & 40 & 7 & $\mathrm{BDL}$ \\
\hline AVL MSS [mg/m^3] & 1.064 & 0.249 & BDL \\
\hline Exhaust Flow Rate $[\mathbf{s c f m}]$ & 273.73 & 250.21 & 281.15 \\
\hline Exhaust Temperature $\left[{ }^{\circ} \mathbf{C}\right]$ & 273.38 & 275.25 & 245.68 \\
\hline
\end{tabular}

In Table 5-3 and Table 5-4, the BDL entries for $\mathrm{CO}$ emissions collected from the Post-2007 and Post-2010 vehicles are resultant of the active DOCs in the systems and subsequent concentration levels at, or below, detection limits (the $\mathrm{CO}$ analyzer was calibrated for the lower limit with $\mathrm{N}_{2}$ with step increase of $\left.1 \mathrm{ppm}\right)$. Active DOCs also explain the low THC concentration levels for those two vehicles. This is not valid in Table 5-2, where high emissions of $\mathrm{CO}$ and $\mathrm{THC}$ are present, mainly related to the lower temperature in the exhaust that does not allow for thermochemical activation of the DOC, usually higher than $275^{\circ} \mathrm{C}$ as showed in Littera et al. (2011) work.

Consistent with the increasingly stringent $\mathrm{NO}_{\mathrm{x}}$ regulations throughout the years of manufacture represented, the $\mathrm{NO}_{\mathrm{x}}$ emissions reported from the Pre-2007 vehicle were the highest. However, Post-2010 $\mathrm{NO}_{\mathrm{x}}$ emissions were reported as being higher than those collected from the Post-2007 vehicle. This disparity is likely due to insufficient exhaust 
thermal energy for optimized SCR performance for both idle and 20mph tests (Table 5-2 and Table 5-3). The lowest $\mathrm{NO}_{\mathrm{x}}$ emissions are observed in Table 5-4 for the Post-2010 truck, where an operational SCR reduced the value within the accuracy of $1 \mathrm{ppm}$ of the analyzer. At higher speeds, the increased load provided increased exhaust temperatures that resulted in much-improved SCR performance. Similar discontinuity with standardsbased intuition was reported for soot sensor data. As indicated, Post-2007 vehicle AVL MSS results were much higher than those collected from the Post-2010 vehicle. Considering that both employed DPFs for PM control, the results should have been comparable. However, the Post-2007 presenting a damaged DPF, positioned itself between the Pre-2007 and Post-2010 in terms of PM emissions in both Table 5-3 and Table 5-4. Interestingly, the increase of PM emissions of the Post-2007 is not linear with the ones of the Pre-2007. This indication reflects a possible saturation for the leaked PM from the cracked DPF. This also confirms that the DPF, even if damaged, was still able to collect sufficient amounts of PM necessary to trigger regeneration activity. PM emissions in Table 5-2 have BDL entries due to values lower than the detectable analyzer limit.

Although, the Post-2007 PM emissions are heavily influenced by the damaged DPF, moving away from ideal working operations, they still represent not a rare case, thus important to assess the real-world emissions for this HDDT.

\subsection{Plume InVestigation}

The evolving plume generated by a HDDT is a dynamic phenomenon in terms of shape and dimension, due to turbulent interaction with the background air. Gaseous 
components undergo a dilution process that does not affect the nature of the kind of emissions themselves. On the other hand, PM emissions due to the rapidity of the dilution and the composition may undergo several changes in terms of particle size distribution and concentration. Such a complex 3D event arose challenges on how to faithfully represent all the key information on a 2D figure.

Contour cut plots of the plume represent a significant source of information to describe the plume evolution and analyze in-homogeneity. Two types of contour cut plots are identified: longitudinal and transverse. The first one is a plane parallel to the longitudinal axis of the wind tunnel $(\mathrm{Z})$ or wind direction, this plane could be either horizontal or vertical. The contours are generated via linear 3D interpolation using the 130 sampling point over a predefined grid. The grid presents a resolution of $10 \mathrm{~mm}$ along $\mathrm{X}$ and $\mathrm{Y}$-axes, and $50 \mathrm{~mm}$ along the $\mathrm{Z}$-axis. In order to reduce calculation time, the interpolating grid is large enough to contain the plume. The transverse contour cut plot is generated upon each sampling plane. This allows for 2D cubic interpolation using the 13 points of each plane. The interpolating grid in this case presents a resolution of $5 \mathrm{~mm}$ in both $\mathrm{X}$ and $\mathrm{Y}$ directions. In addition, the interpolating grid is large enough to contain the plume. The total PM count contour plots are generated by summation of the tailpipecorrected EEPS ${ }^{\circledR}$ channels. The tailpipe correction is obtained by correcting the sampling points by the local DR. This step is fundamental in comparing different points of the plume.

As already described, the local DR is the ratio between concentration of raw exhaust and plume locale of a gaseous component. $\mathrm{NO}_{\mathrm{x}}$ based $\mathrm{DR}$ resulted more accurate for Pre-2007 and Post-2007, because of nature of the emission of this two HDDTS and the better performances of the $\mathrm{NO}_{\mathrm{x}}$ analyzer if compare to the $\mathrm{CO}_{2}$ one. The Post-2010 HDDT shows very low $\mathrm{NO}_{\mathrm{x}}$ emissions, thus the $\mathrm{CO}_{2}$ based DR resulted more reliable. Contour plots for the PM account for the total PM count, accumulation mode PM count, and 
nucleation mode PM count. The accumulation and nucleation mode separation is defined at $30 \mathrm{~nm}$. Generally, in a double mode particle size distribution and concentration, this separation takes place between 20 and 50nm, thus $30 \mathrm{~nm}$ represents the middle of this range. This approach is necessary to identify regions in which the nucleation mode or accumulation mode is predominant. The last type of plot is a typical particle size distribution and concentration meant to compare different sampling points of the plume. The channels selected for both 3D and 2D interpolating contour cut plots are:

- $\mathrm{CO}_{2}[\%]$

- $\mathrm{NO}_{\mathrm{x}}[\mathrm{ppm}]$

- Wind Speed $[\mathrm{m} / \mathrm{s}]$

- Turbulence Intensity [\%]

- $\mathrm{NO}_{\mathrm{x}}$ based Dilution Ratio

- Temperature $[\mathrm{K}]$

- DustTrack $\left[\mathrm{mg} / \mathrm{m}^{3}\right]$

- Aethalometer $\left[\mathrm{ng} / \mathrm{m}^{3}\right]$

- Total PM Count $\left[\# / \mathrm{cm}^{3}\right]$

- Nucleation Mode PM Count $\left[\# / \mathrm{cm}^{3}\right]$

- Accumulation Mode PM Count $\left[\# / \mathrm{cm}^{3}\right]$

\subsubsection{Pre-2007 Vehicle Test Results}

This section shows the results of the Pre-2007 vehicle, analyzing the shape and dimension of the plume. Additionally, the goal is to understand how the PM emissions evolve within the plume itself. 


\subsubsection{Idle Test}

As described previously in the idle test, the vehicle is in idling condition and a wind of 5mph is generated. This test represents the emissions of the truck in a rest area. Table 55 summarizes the vehicle and dynamometer parameter during the idle test for the Pre-2007 vehicle.

Table 5-5: Vehicle and Dynamometer Parameter during the idle test for the Pre-2007 Vehicle.

$\begin{array}{ll}\text { Parameter Value } & \text { Val }\end{array}$

Dynamometer Torque [ft-lb]

Dynamometer Power [hp]

Dynamometer Speed [mph]

Engine Speed [RPM] $\quad 850$

Transmission gear 


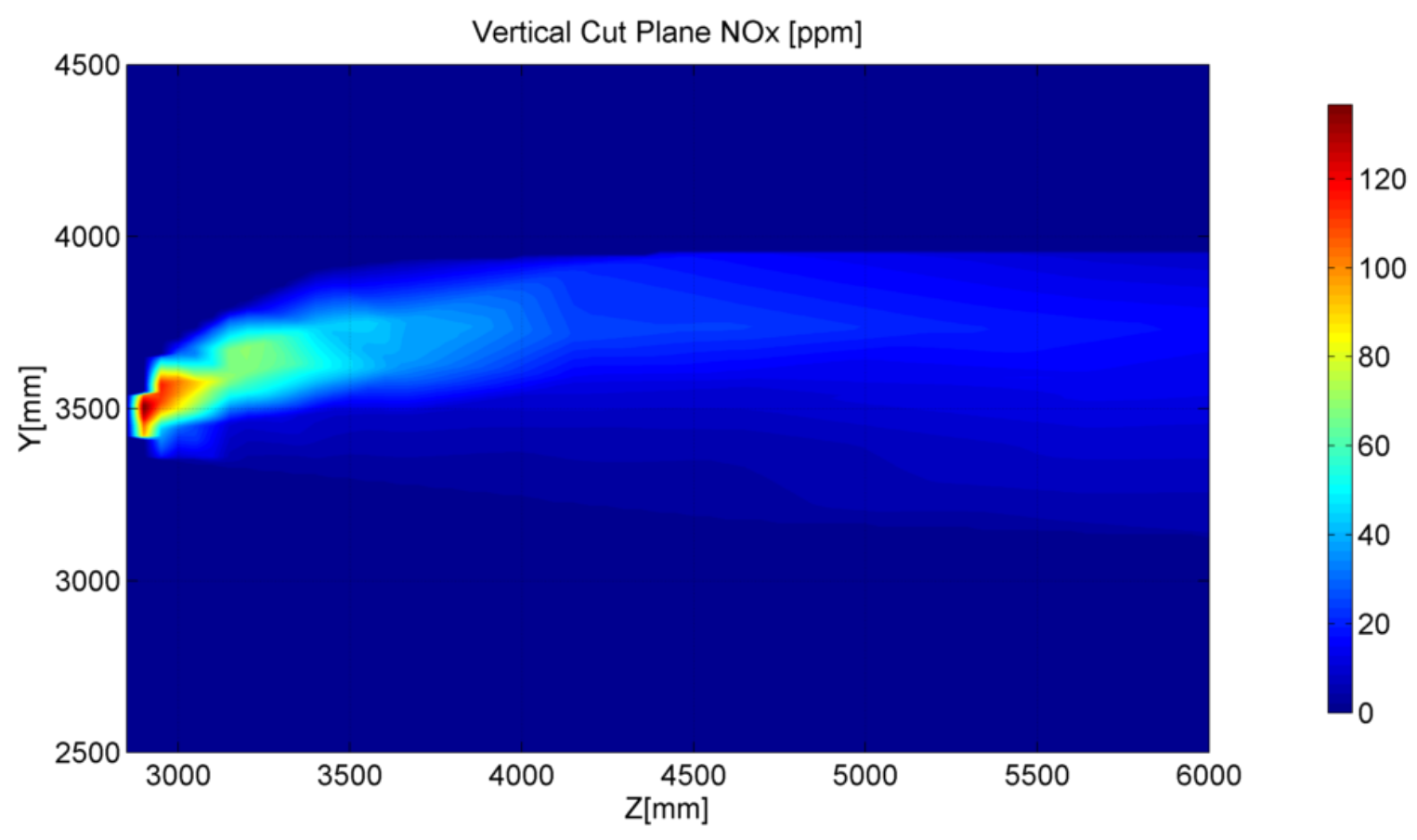

Figure 5-2: Longitudinal vertical contour cut plot of the $\mathrm{NO}_{\mathrm{x}}$ trace for the Pre-2007 vehicle during the idle test. The plane is passing through the exhaust stack.

Figure 5-2 shows the longitudinal vertical contour cut plot of the $\mathrm{NO}_{\mathrm{x}}$ trace for the Pre-2007 vehicle during the idle test. The left side of the plot is found immediately after the cabin, while the right side is downwind. The cut plot passes through the exhaust stack. The plume is defined as the area where the $\mathrm{NO}_{\mathrm{x}}$ concentration is greater than zero. The maximum concentration value of $130 \mathrm{ppm}$ is observed close to the exhaust stack, agreeing with $213 \mathrm{ppm}$ identified in the raw exhaust. This indicates that the dilution process starts within a few centimeters from of the emitting point. The beginning of the plume observes a faster dilution, which slows down further away from the emitting point. The core of the plume undergoes a slower dilution if compared to the outer regions. In detail, the lower outer region has faster dilution than the upper region. This result can be better understood with Figure 5-3, showing that the bottom part of the plume is within the high turbulence 
region of the truck wake. On the other hand, the upper region is outside the truck wake. This difference leads to a similar alteration in dilution conditions for the regions, since TI is an indicator of dilution rate. The TI tends to decrease further downwind due to a dissipation process intrinsic of turbulent flows, which explains the reduction in dilution rate of the plume.

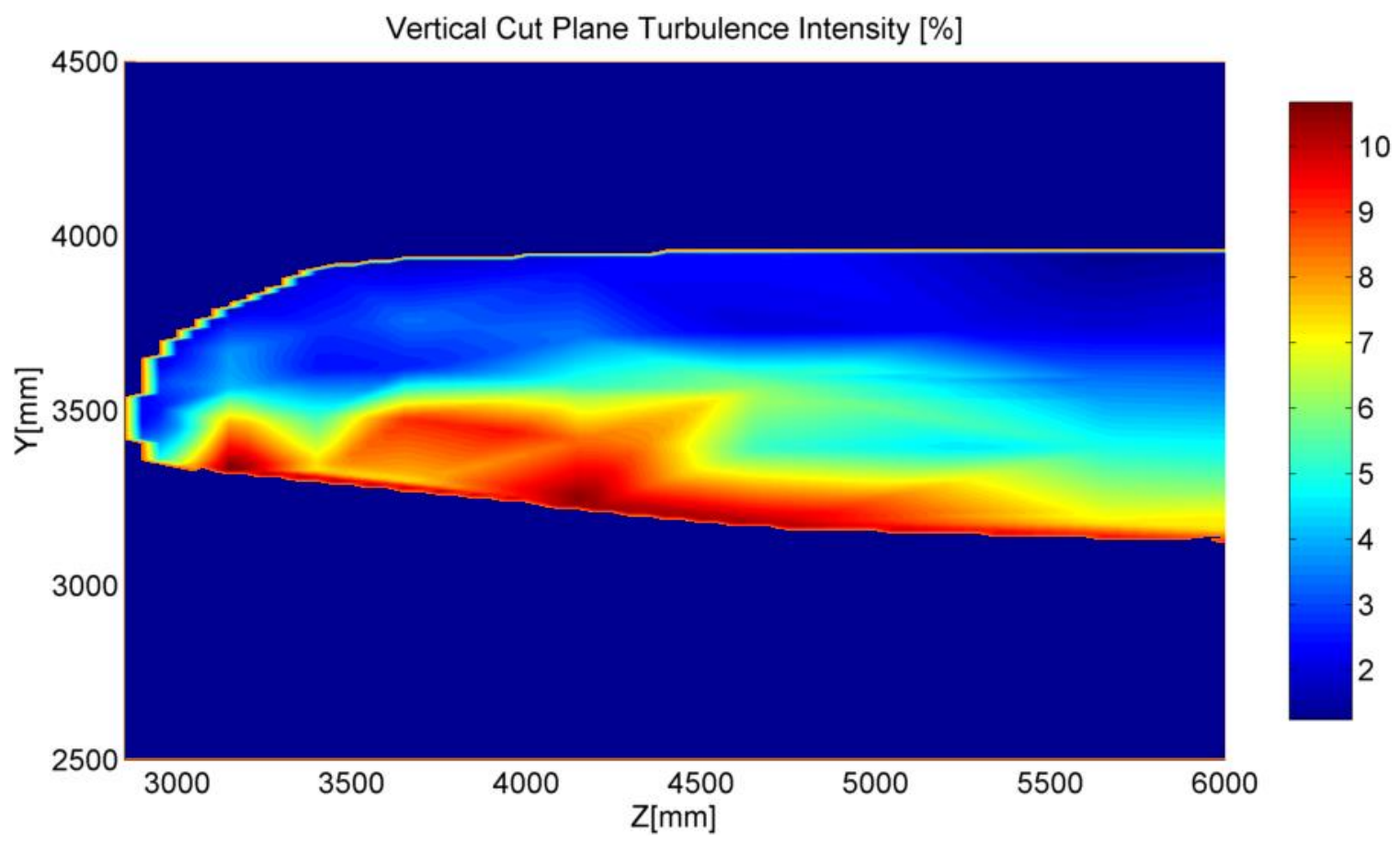

Figure 5-3: Longitudinal vertical contour cut plot of the Turbulence Intensity trace for the Pre-2007 vehicle during the idle test. The plane is passing through the exhaust stack.

Figure 5-3 also illustrates how the wake generated by the truck tends to move downward, which is explained by the transfer of momentum from upper free stream. The plume core observes a slower dilution and cooling process by the combination of two affects, if compared to the outer regions. The first effect is one that is less exposed to the 
background air, while the second is related to the position of the core while outside the turbulent truck wake.
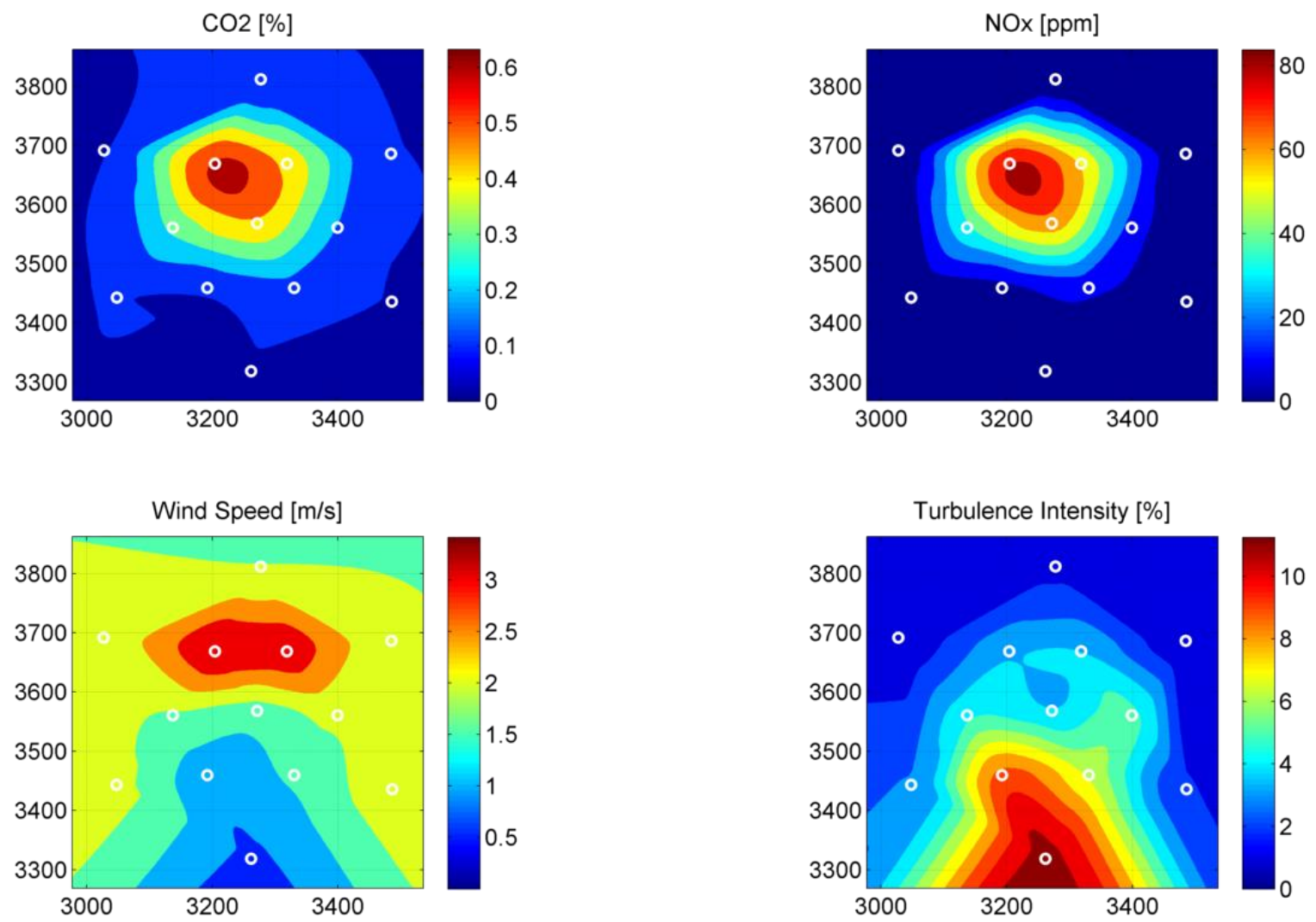

Figure 5-4: Transverse contour cut plot of $\mathrm{CO}_{2}, \mathrm{NOx}$, wind speed, and TI. The plane of this plot is sampling plane 2 , which is $0.315 \mathrm{~m}$ from the emitting point for the idle test of the Pre-2007 vehicle. The white dots represent the defining plane sampling points.

Analyzing Figure 5-4, it is important to note that the $\mathrm{CO}_{2}$ and $\mathrm{NO}_{\mathrm{x}}$ contours are similar in shape, dimension, and gradient. This information guarantees the cross validation of the analytical instruments, reassuring the plume identification tools. The wind speed and TI plots of Figure 5-4 indicate that outside the plume, the wind speed is $2 \mathrm{~m} / \mathrm{s}$ and the TI is 
$1.7 \%$, confirming upstream cabin measurements. In addition, the wind speed and TI plots of Figure 5-4 confirm the bottom outer region of the plume is immersed in the truck wake, while the upper is exposed to free wind stream. The lower region experiences higher TI and lower wind speed, while the upper region experiences lower TI and higher wind speed. Dilution rate and cooling rate are directly related to the mixing rate, which are well represented by the TI. It is crucial to investigate how this mixing heterogeneity can affect the PM, since nucleation mode formation and evolution are strongly related to dilution and super-saturation conditions.

The DustTrack is an aerosol sampling device that provides mass based PM via light-scattering technology for air quality pollution control. Due to the greater optical properties of solid particles over organic particles, it is safe to indicate that the contour plot of the DustTrack provides information for the solid compound of the PM (Ng, Ma, \& Kittelson, 2007). In addition, this is confirmed that solid particles represent the majority of the PM mass (Kittlelson, 1998). For the abovementioned, the DustTrack plot of plane 2 ( $0.315 \mathrm{~m}$ from exhaust stack) in Figure 5-5 resembles the $\mathrm{CO}_{2}$ and $\mathrm{NO}_{\mathrm{x}}$ contours in Figure 5-4. The total PM count corrected to tailpipe does not agree with the previous plot due to the effect of the dilution on the particle size distribution and concentration.

The first question to answer is if within the plume, is a single mode or double mode distribution is present? Splitting the total PM count into nucleation and accumulation mode, the nucleation mode concentration peak differs from that of the accumulation mode. The nucleation mode peak has a greater value than the accumulation mode, and it is toward the region with higher turbulence. The nucleation mode peak presents a count mean diameter of $15 \mathrm{~nm}$ (typical for a double mode particle size distribution and concentration). The peak in accumulation mode instead is placed in the core of the plume with a CMD of $122 \mathrm{~nm}$ (typical for diesel emissions without DPF). This distinct separation of the accumulation 
mode and nucleation mode peaks is a plausible indication on how the different dilution rates in different areas of the plume can enhance the nucleation mode formation in the early stages of the plume.
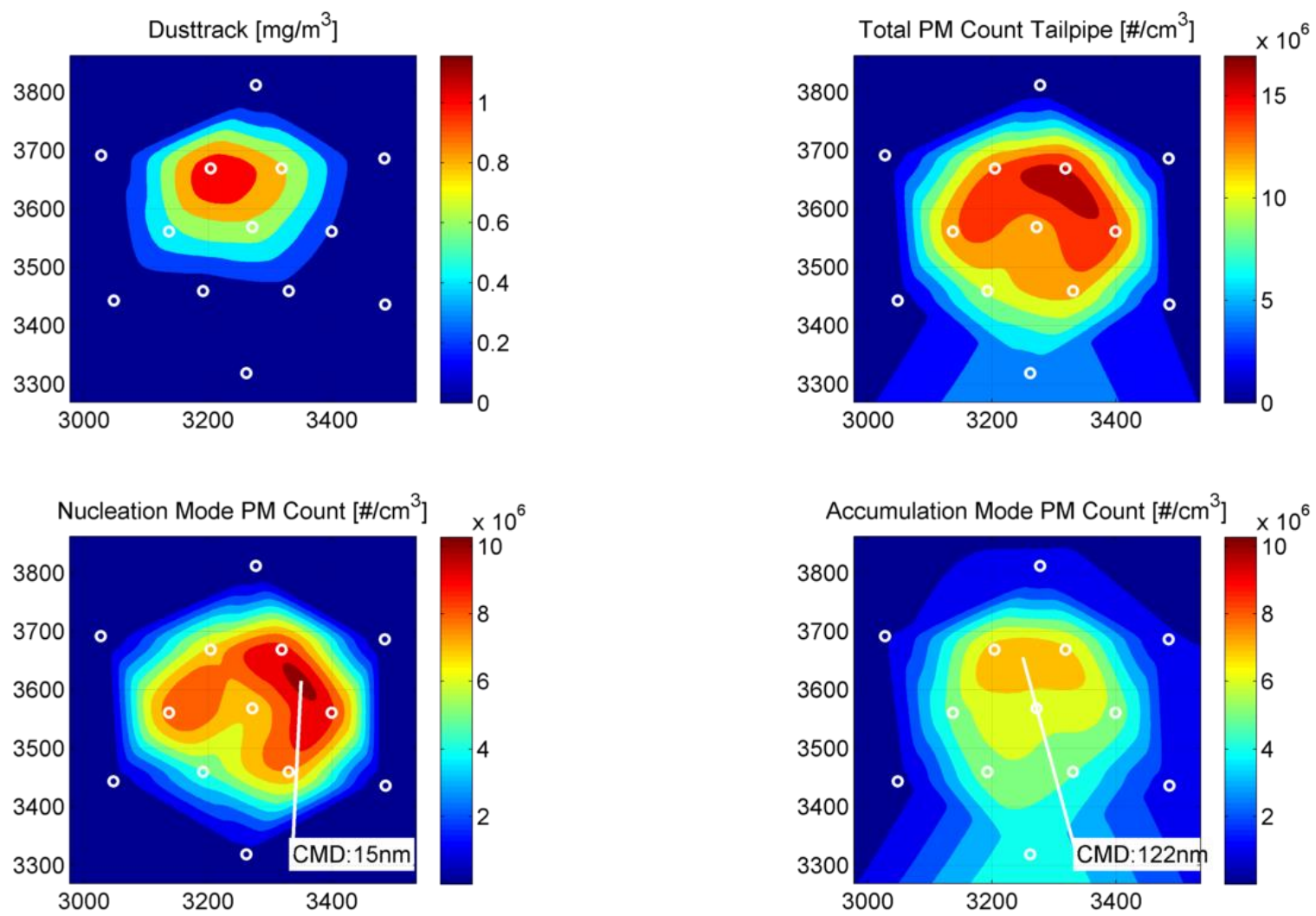

Figure 5-5: Transverse contour cut plot of DustTrack, total PM Count tailpipe corrected, nucleation mode PM count, and accumulation mode PM count for the idle test of the Pre-2007 vehicle. The plane of this plot is sampling plane 2, which is $0.315 \mathrm{~m}$ from the emitting point. The white dots represent the plane sampling points. 
Figure 5-6 shows the particle size distribution of point 12 on plane 2 (closest to the accumulation mode peak) and point 13 on the same plane (closest to the nucleation mode peak). A slight, but evident difference in nucleation mode is present, while the accumulation mode overlaps. Both traces are represented with standard deviation error bars.

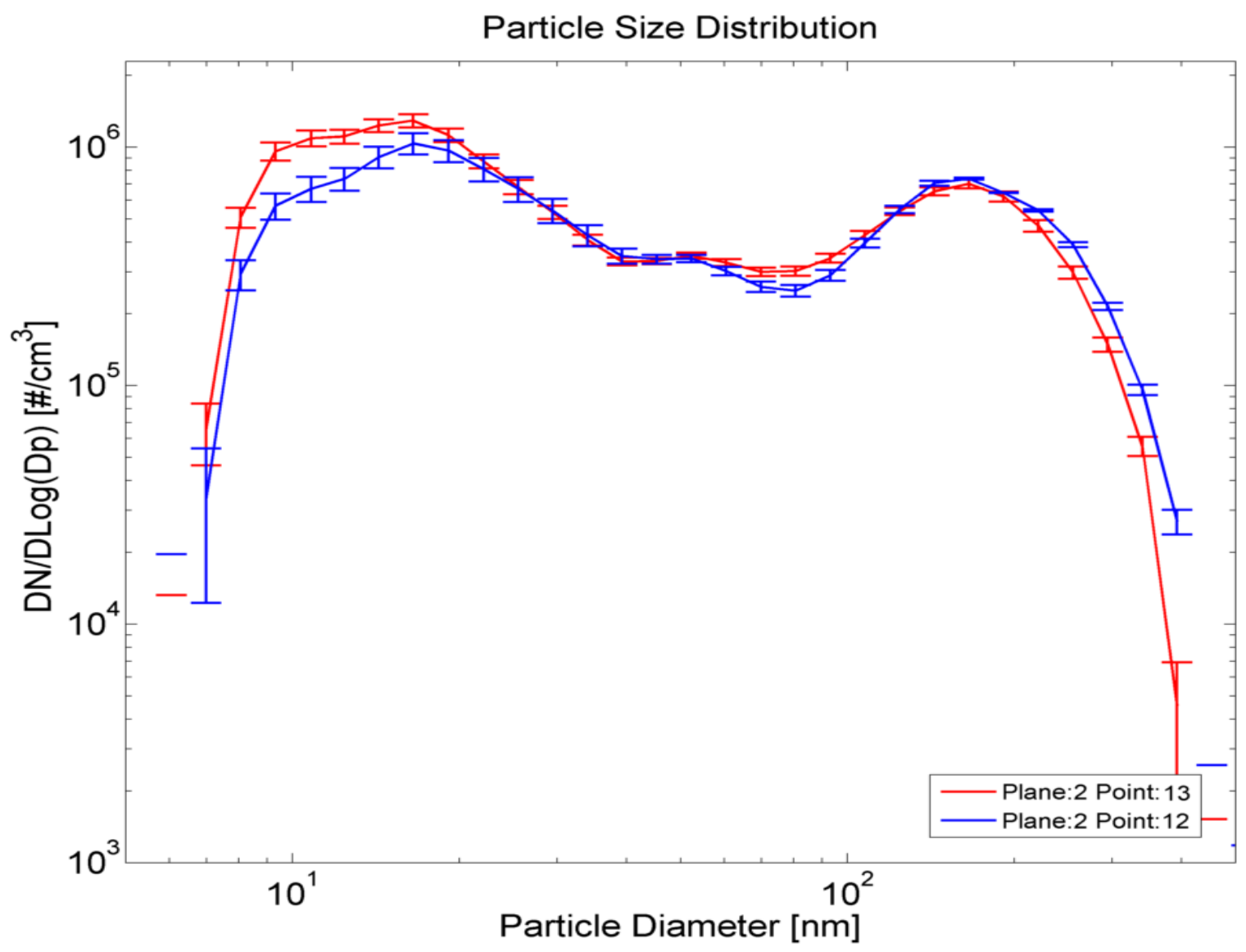

Figure 5-6: Particle size distribution of point 12 and point 13 of plane $2(0.315 \mathrm{~m}$ downstream) for the idle test of the Pre-2007 vehicle.

In the early stages of the plume, two distinct peaks for accumulation and nucleation mode are present. Moving further downstream, the continuous turbulent mixing added by 
a slow dissipation process causes those two distinct areas to merge, the particle size distribution and concentration have similar profile and magnitude.
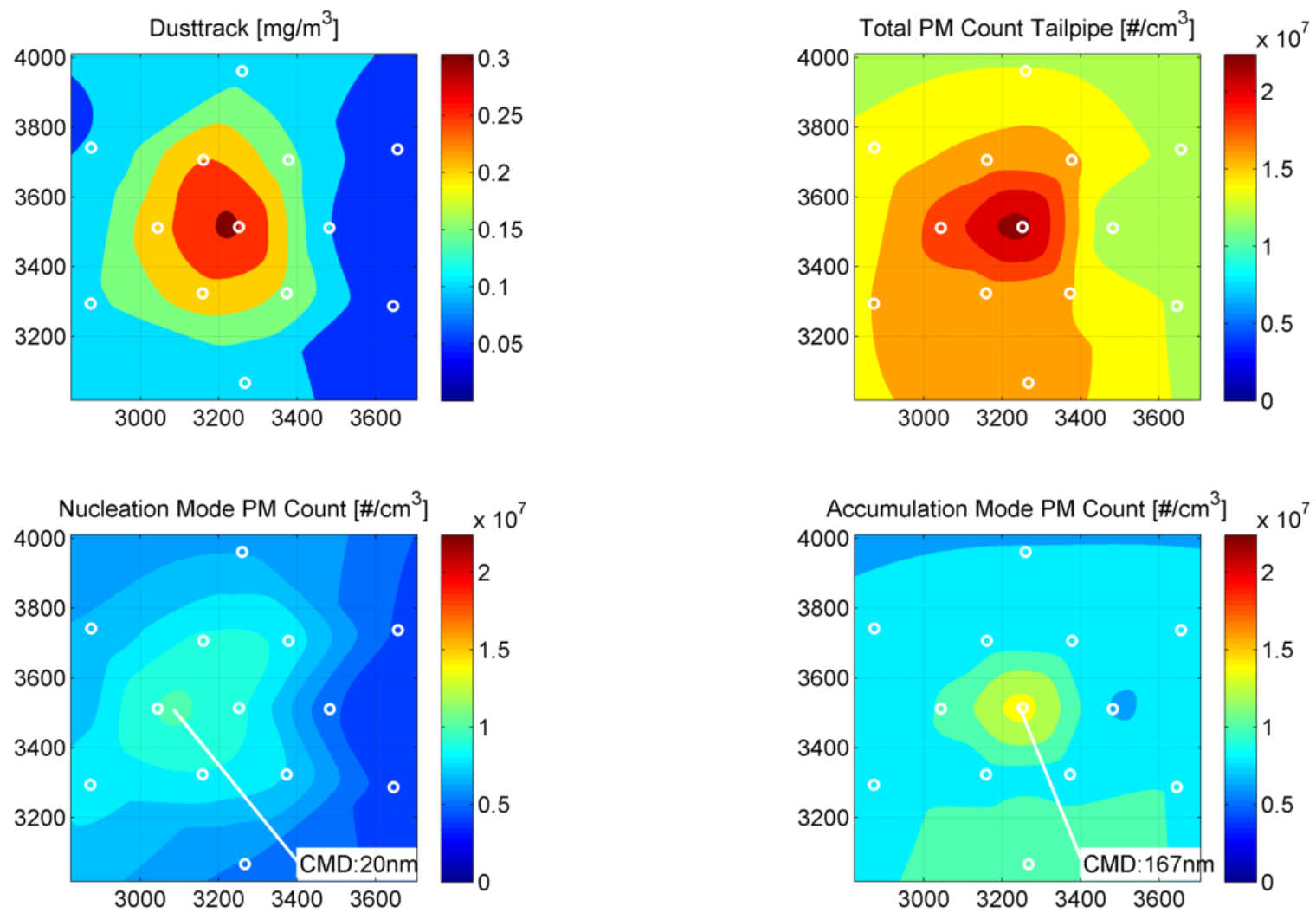

Figure 5-7: Transverse contour cut plot of DustTrack, total PM count tailpipe corrected, nucleation mode PM count, and accumulation mode PM count for the idle test of the Pre-2007 vehicle. The plane of this plot is sampling plane 9, which is $6.815 \mathrm{~m}$ from the emitting point. The white dots represent the plane sampling points.

The accumulation and nucleation mode PM count of Figure 5-7 are similar for plane 9, which is $6.815 \mathrm{~m}$ downstream of the exhaust stack. This is a good indication on how an initial heterogenic PM particle size distribution and concentration is more homogenous. 
Figure 5-8 confirms there are almost identical particle size distributions and concentrations for three different points of the plume. The accumulation mode from the particle size distribution of plane 2 (see Figure 5-6) and plane 9 (see Figure 5-8) are very similar, indicating that no major changes occurred on the larger particles. On the other hand, the nucleation mode observed an appreciable variation in $\mathrm{CMD}$, from $15 \mathrm{~nm}$ in plane 2 , to $20 \mathrm{~nm}$ in plane 9; while the peak concentration is constant at $1 \times 10^{6} \# / \mathrm{cm}^{3}$. Such transformation is explainable with a coagulation or absorption of the nucleation mode particles.

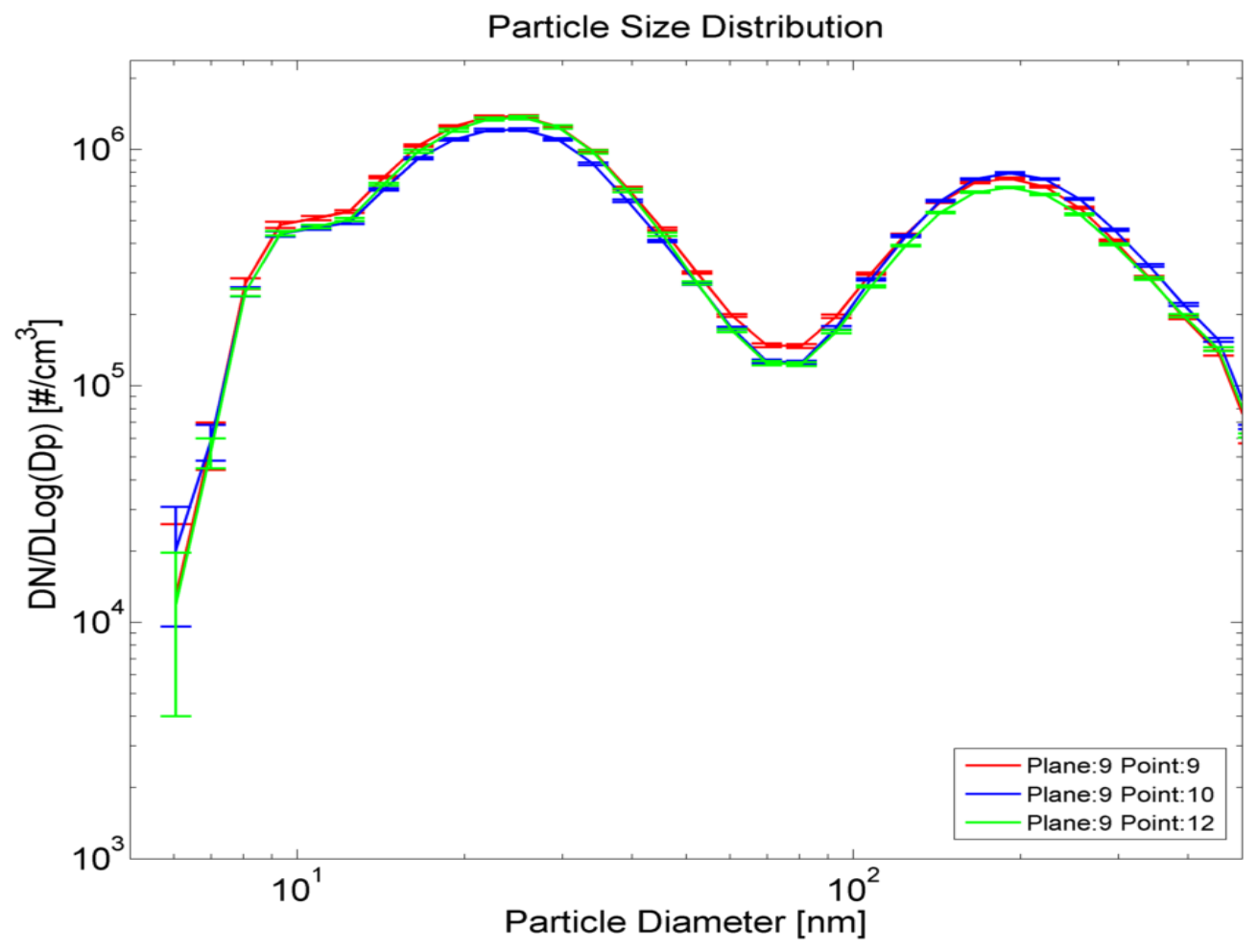

Figure 5-8: Particle size distribution of point 9 , point 10, and point 12 of plane $9(6.815 \mathrm{~m}$ downstream) for the idle test of the Pre-2007 vehicle. 


\subsubsection{20mph Test}

As described previously in the $20 \mathrm{mph}$ test, the vehicle and wind speed are matched at $20 \mathrm{mph}$. This test represents a HDDT traveling at $20 \mathrm{mph}$ on a flat road in calm wind. The road load equation indicates that the total power required at the wheel is $40 \mathrm{hp}$. Table $5-6$ summarizes the vehicle and dynamometer parameter during the $20 \mathrm{mph}$ test for the Pre2007 vehicle.

Table 5-6: Vehicle and Dynamometer Parameter During the 20mph Test for the Pre-2007 Vehicle.

\begin{tabular}{lc}
\hline Parameter & Value \\
\hline Dynamometer Torque [ft-lb] & 300 \\
\hline Dynamometer Power [hp] & 40 \\
\hline Dynamometer Speed [mph] & 20 \\
\hline Engine Speed [RPM] & 1400 \\
\hline Transmission gear & 7 th \\
\hline
\end{tabular}

The plume for this wind speed results more elongated and had a tubular shape in comparison to the idle test (see Figure 5-9), which is mainly related to higher wind speed and by a consistent increase in exhaust flow rate. The raw exhaust emission of $\mathrm{NO}_{\mathrm{x}}$ accounts for $892 \mathrm{ppm}$ and the maximum observed in Figure 5-9 is 510ppm, confirming that dilution takes place right at the exit of the exhaust stack. Comparing Figure 5-9 and Figure $5-10$, during this test the plume is completely entrained within the wind cowl wake. The turbulence experiences a faster decay, speculation is on a higher Reynolds number in this test. Contour plots for gaseous components confirm the identification of the plume with a 
tubular shape (see Figure 5-11) and the presence of higher TI and lower wind speed for the lower peripheral region of the plume. While, the upper region experiences higher wind speed and lower TI.

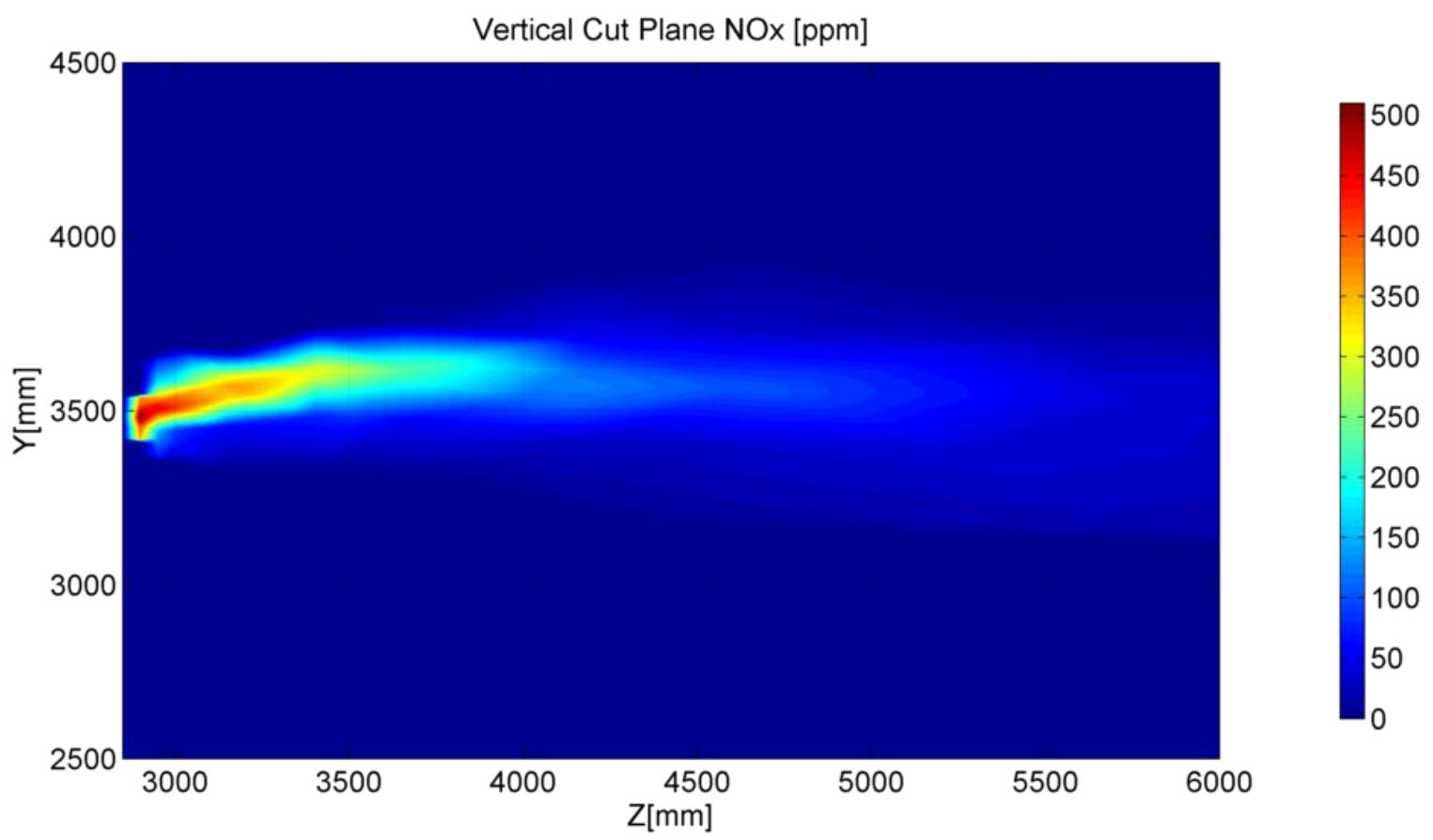

Figure 5-9: Longitudinal vertical contour cut plot of the NOx trace for the Pre-2007 vehicle during the $20 \mathrm{mph}$ test. The plane is passing through the exhaust stack.

Similar to what was observed in the idle test, the total PM count tailpipe corrected does not match the gaseous contours. The accumulation and nucleation mode experience two separated peaks; the accumulation mode peak is in proximity of the core of the plume, while the second is in a higher TI area (see Figure 5-11 and Figure 5-12). This case, due to the higher wind speed and higher PM production by the engine, PM emissions and the effect of TI on it are easier to observe. 


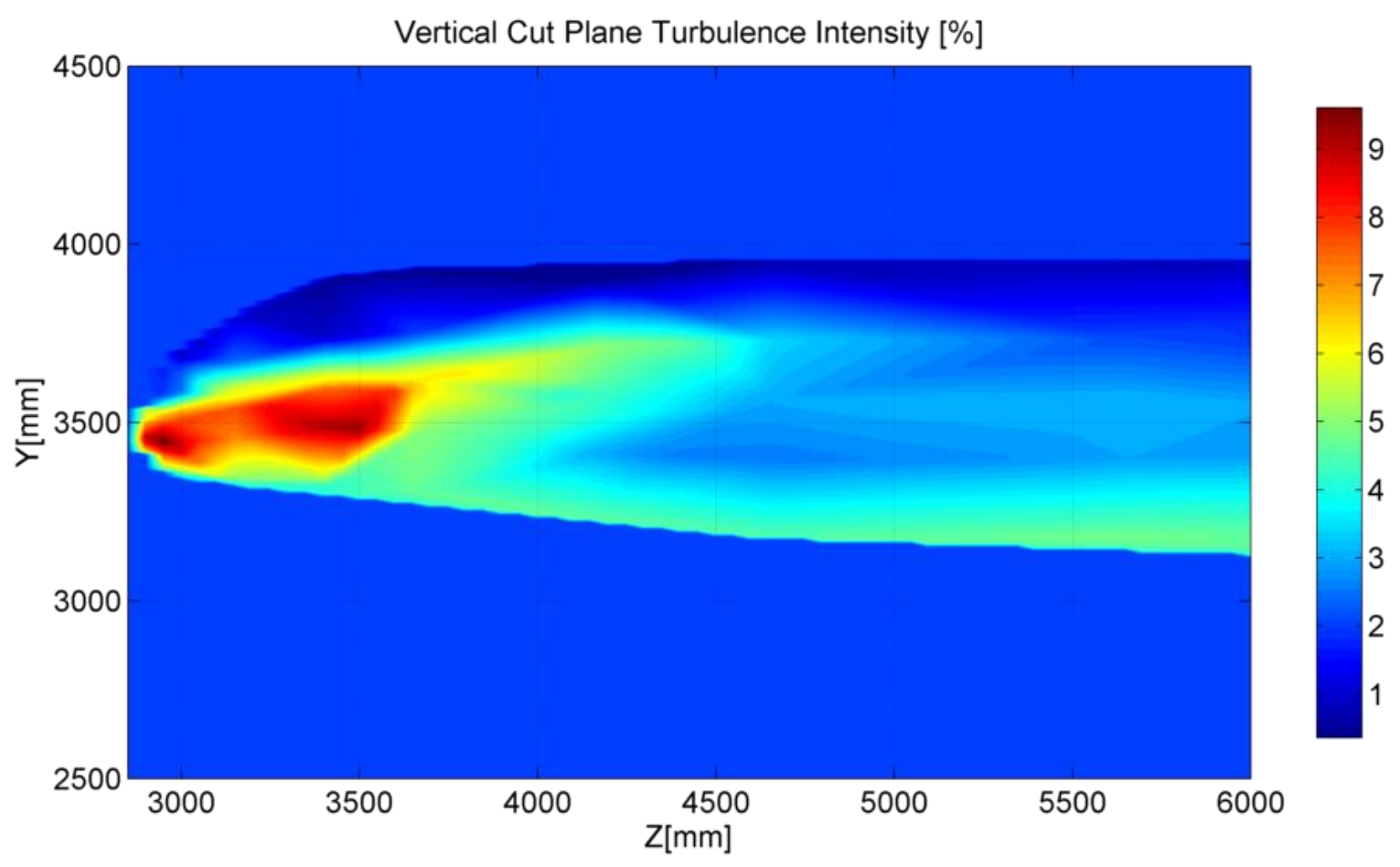

Figure 5-10: Longitudinal vertical contour cut plot of the turbulence intensity trace for the Pre-2007 vehicle during the $20 \mathrm{mph}$ test. The plane is passing through the exhaust stack.

The nucleation mode peak expresses a CMD of $17 \mathrm{~nm}$ while the accumulation mode indicates a CMD of 39nm (see Figure 5-12), which does not match with a typical value for an accumulation mode in double mode distribution. Investigation on the particle size distribution and concentration of point 1 (closest to the accumulation mode peak) and point 10 (closest to the nucleation mode peak) of plane $1(0.115 \mathrm{~m}$ downstream), indicates that point 1 presents a single mode distribution. A plausible explanation elucidates that point 1 has not undergone any significant dilution capable of nucleation mode generation (see Figure 5-13). 

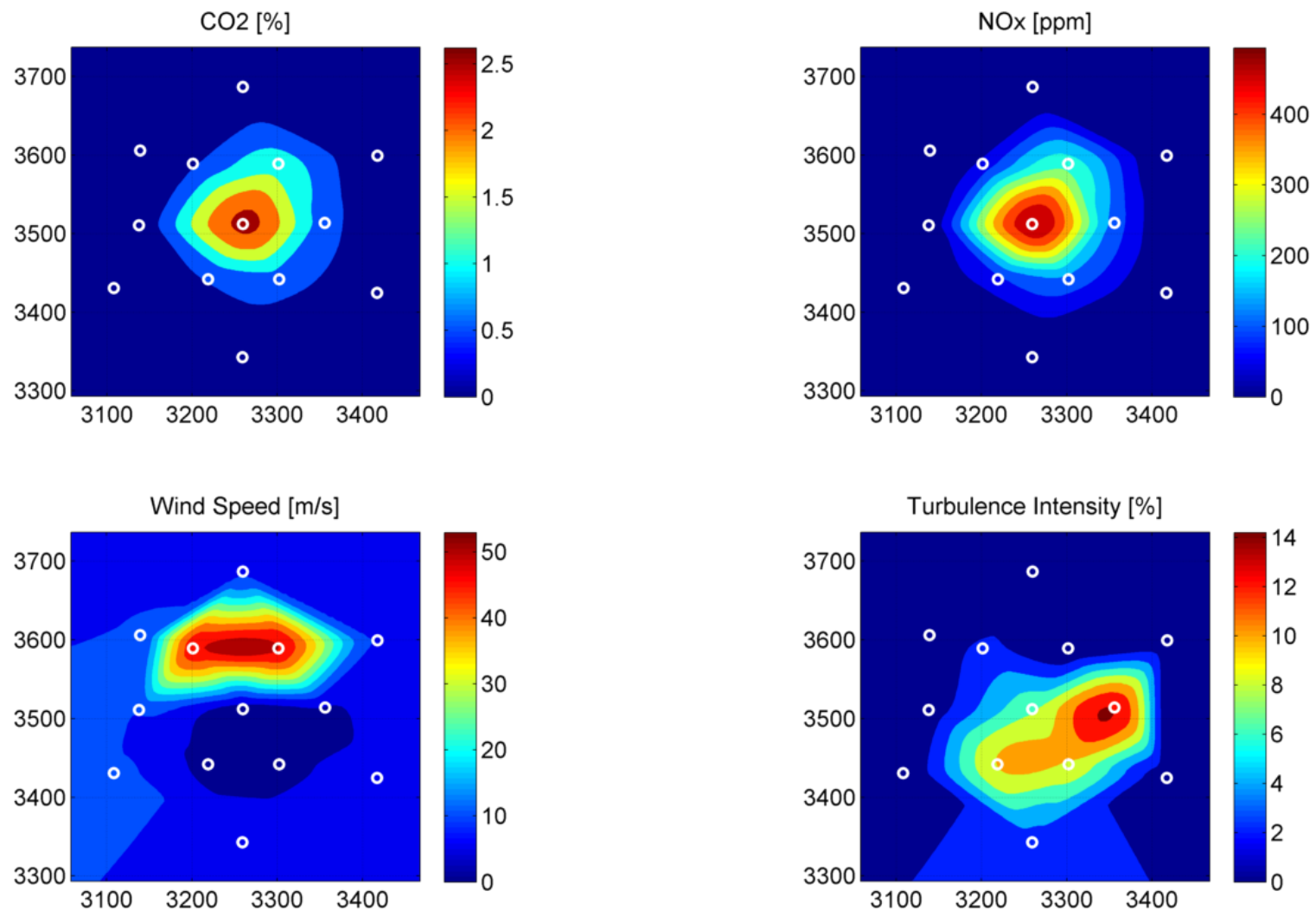

Figure 5-11: Transverse contour cut plot of CO2, NOx, wind speed, and TI. The plane of this plot is sampling plane 1 , which is $0.115 \mathrm{~m}$ from the emitting point for the 20mph test of the Pre-2007 vehicle. The white dots represent the defining plane sampling points.

On the other hand, point 10 experiencing a higher TI and dilution rate presents a predominant nucleation mode. At plane 7 (2.815m from emitting point), the turbulence has homogenized the wind speed and TI as well the PM emissions (see Figure 5-14 and Figure 5-15). This result is clear from Figure 5-16 showing that a complete mixing took place between the regions that had predominant nucleation and accumulation mode. A similar particle size distribution and concentration is observable across the entire plume. The 
nucleation mode in this test results one order of magnitude higher than the previous test, mainly related to the higher load required by the engine.
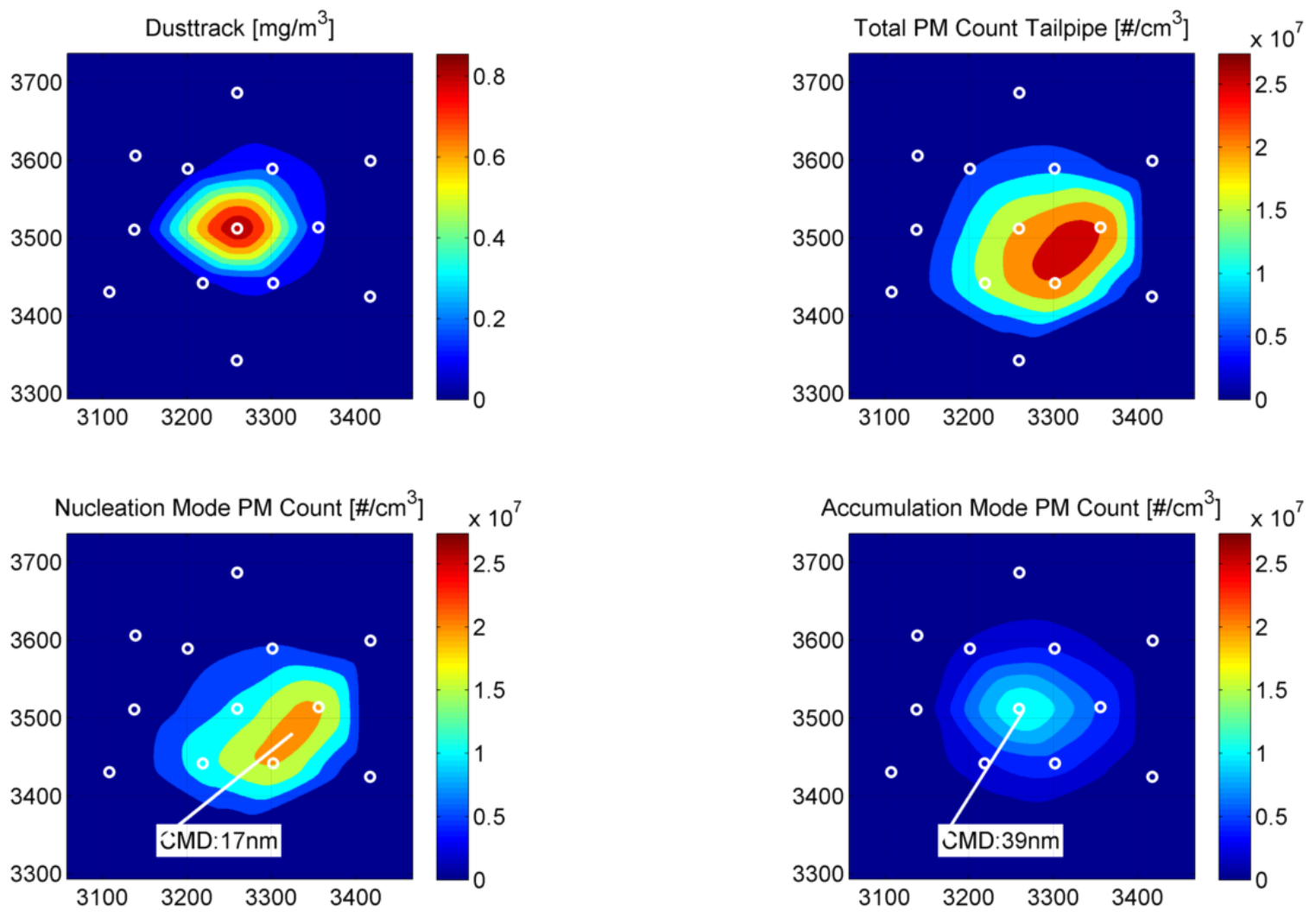

Figure 5-12: Transverse contour cut plot of DustTrack, total PM Count tailpipe corrected, nucleation mode PM count, and accumulation mode PM count for the $20 \mathrm{mph}$ test of the Pre-2007 vehicle. The plane of this plot is sampling plane 1, which is $0.115 \mathrm{~m}$ from the emitting point. The white dots represent the plane sampling points. 


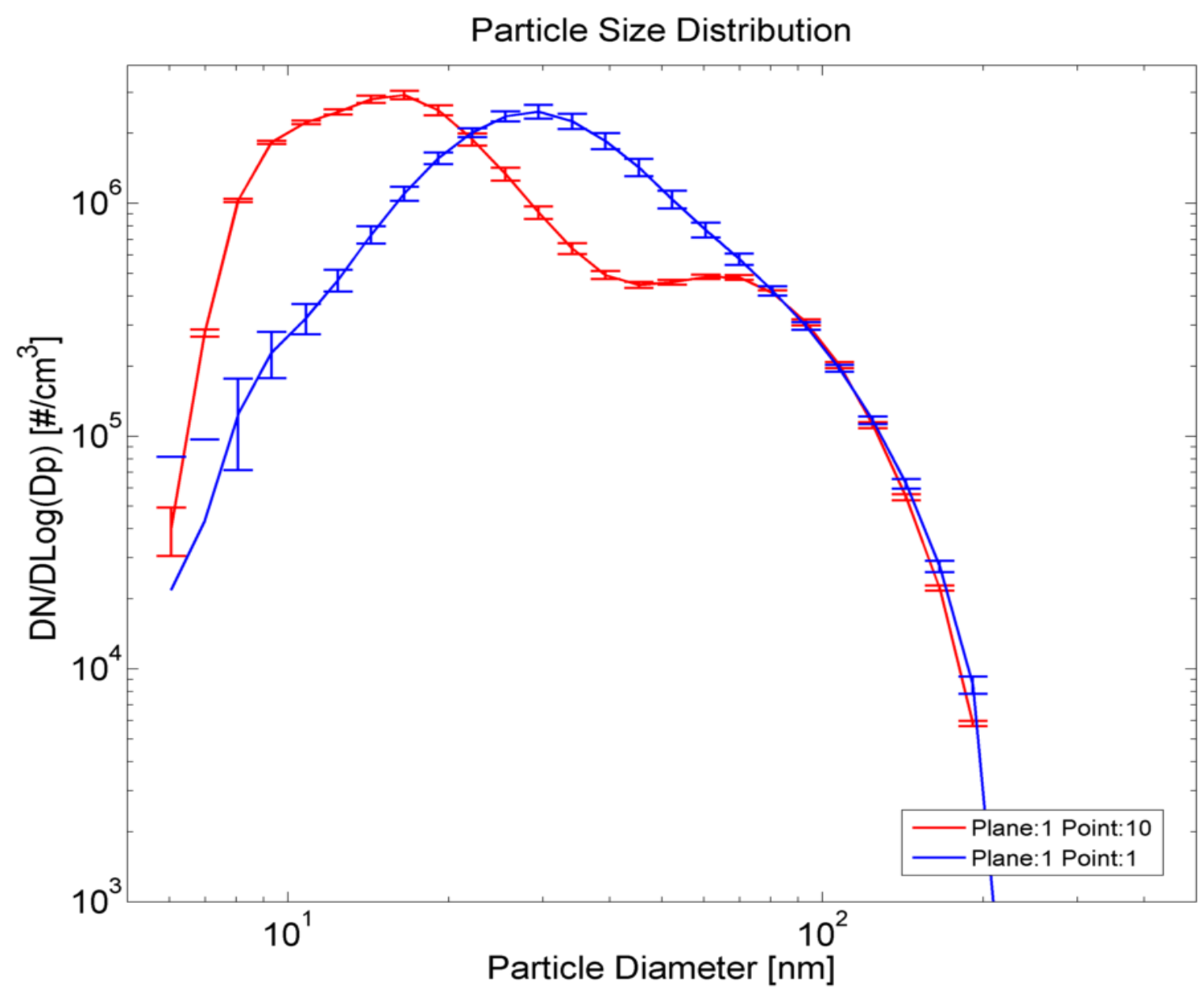

Figure 5-13: Particle size distribution of point 1 and point 10 of plane $1(0.115 \mathrm{~m}$ downstream) for the $20 \mathrm{mph}$ test of the Pre-2007 vehicle. 

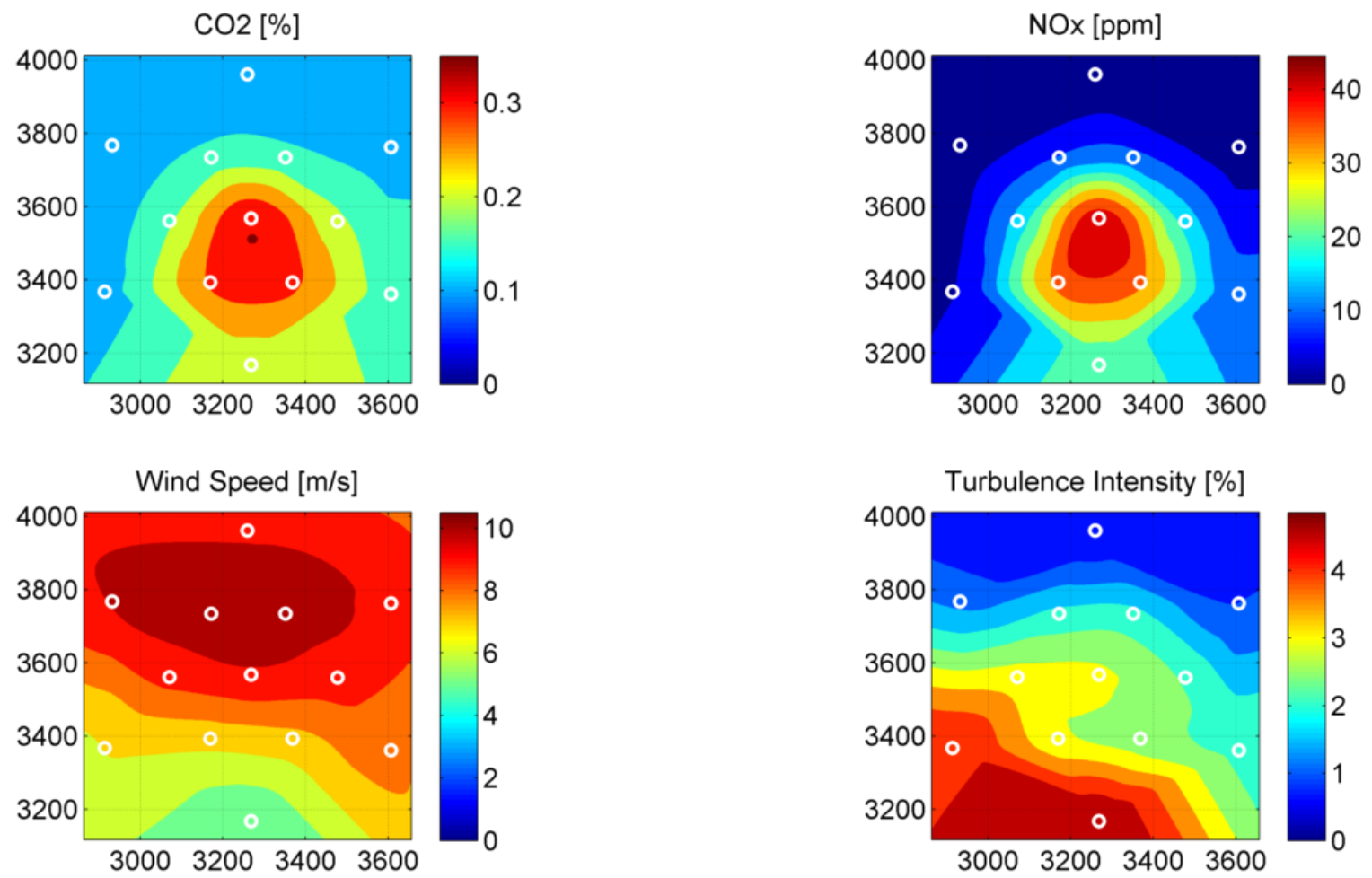

Figure 5-14: Transverse contour cut plot of $\mathrm{CO}_{2}$, NOx, wind speed, and TI. The plane of this plot is sampling plane 7 , which is $2.815 \mathrm{~m}$ from the emitting point for the $20 \mathrm{mph}$ test of the Pre-2007 vehicle. The white dots represent the defining plane sampling points. 

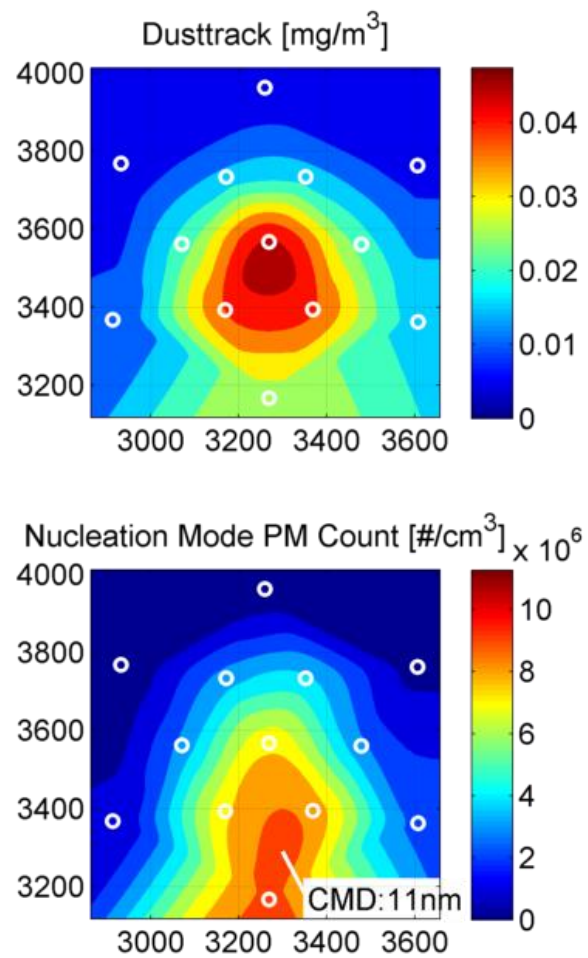
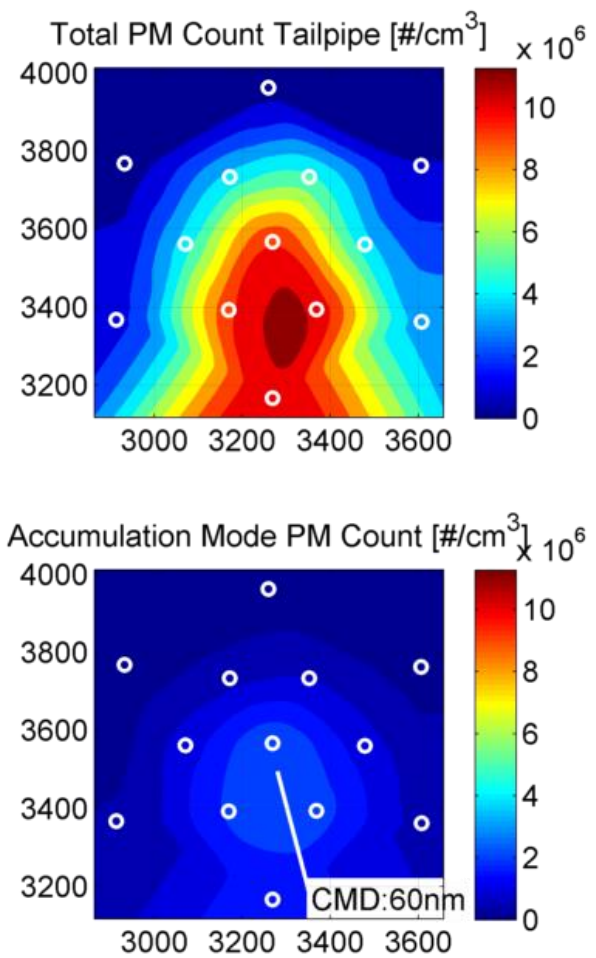

Figure 5-15: Transverse contour cut plot of DustTrack, total PM count tailpipe corrected, nucleation mode PM count, and accumulation mode PM count for the $20 \mathrm{mph}$ test of the Pre-2007 vehicle. The plane of this plot is sampling plane 7, which is $2.815 \mathrm{~m}$ from the emitting point. The white dots represent the plane sampling points. 


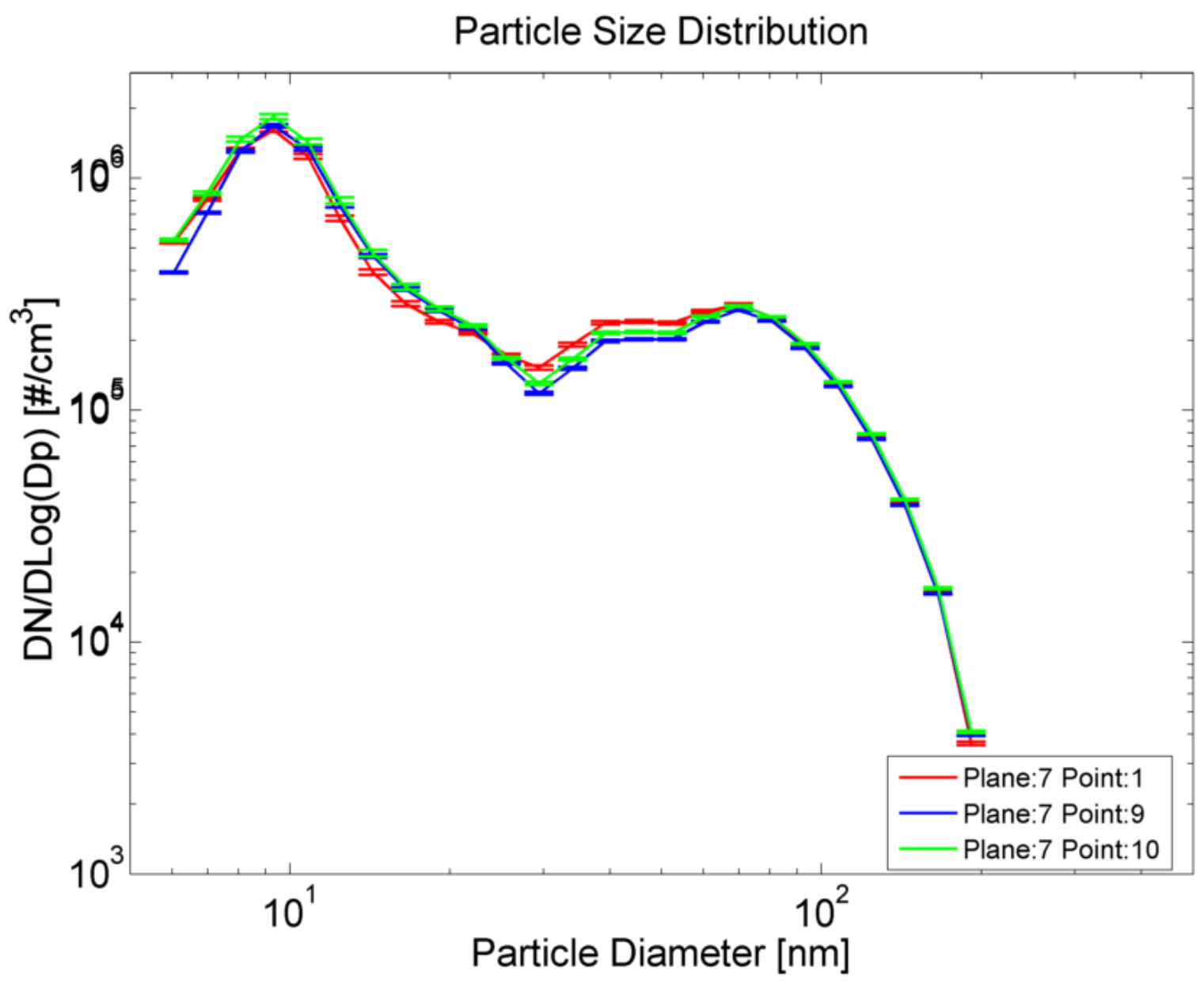

Figure 5-16: Particle size distribution of point 1, point 9, and point 10 of plane $7(2.815 \mathrm{~m}$ downstream) for the $20 \mathrm{mph}$ test of the Pre-2007 vehicle. 


\subsubsection{35mph Test}

As described previously in the $35 \mathrm{mph}$ test, the vehicle and wind speed are matched at $35 \mathrm{mph}$. This test represents a HDDT traveling at $35 \mathrm{mph}$ on a flat road in calm air. The road load equation indicates that the total power required at the wheel is $63 \mathrm{hp}$. Table $5-7$ summarizes the vehicle and dynamometer parameter during the $35 \mathrm{mph}$ test for the Pre2007 vehicle.

Table 5-7: Vehicle and Dynamometer Parameter During the 35mph Test for the Pre-2007 Vehicle.

\begin{tabular}{lc}
\hline Parameter & Value \\
\hline Dynamometer Torque [ft-lb] & 300 \\
\hline Dynamometer Power [hp] & 63 \\
\hline Dynamometer Speed [mph] & 35 \\
\hline Engine Speed [RPM] & 1200 \\
\hline Transmission gear & 9 th \\
\hline
\end{tabular}

The high energy content in the wind for the $35 \mathrm{mph}$ test induces an extremely rapid dilution and cooling of the exhaust with background air. The plume presents a smaller longitudinal component, much less than the $20 \mathrm{mph}$ case, as shown in see Figure 5-17. A TI peak of $16 \%$ is a noticeable indication of the intensity of mixing and dilution happening in the plume. In the $20 \mathrm{mph}$ test, the TI peak was $9 \%$ as illustrated in Figure 5-18. The wind cowl edge with high speed generates higher TI than the cabin wake. In addition, the shortening of the longitudinal plume dimension should account that the $\mathrm{NO}_{\mathrm{x}}$ peak of 
$875 \mathrm{ppm}$ at the stack exhaust outlet is almost double the previous case. Due to the wind cowl deflection effect, the turbulent region completely engulfed the plume. The combination of higher wind speed and higher load generated a pattern of easy identification. On one side, higher TI would lead to greater chances of formation and growth of nucleation mode. But on the other side, higher load reduced the concentration of THC in the exhaust and increased soot formation. The result is a smaller nucleation mode (compared to idle and 20mph test) with an enlarged accumulation mode. More properly, the observable particle size distribution and concentration is a single mode. Figure 5-20 shows how different points across the plume at plane $5(1.315 \mathrm{~m}$ downwind the emitting point) show similar single mode distribution.

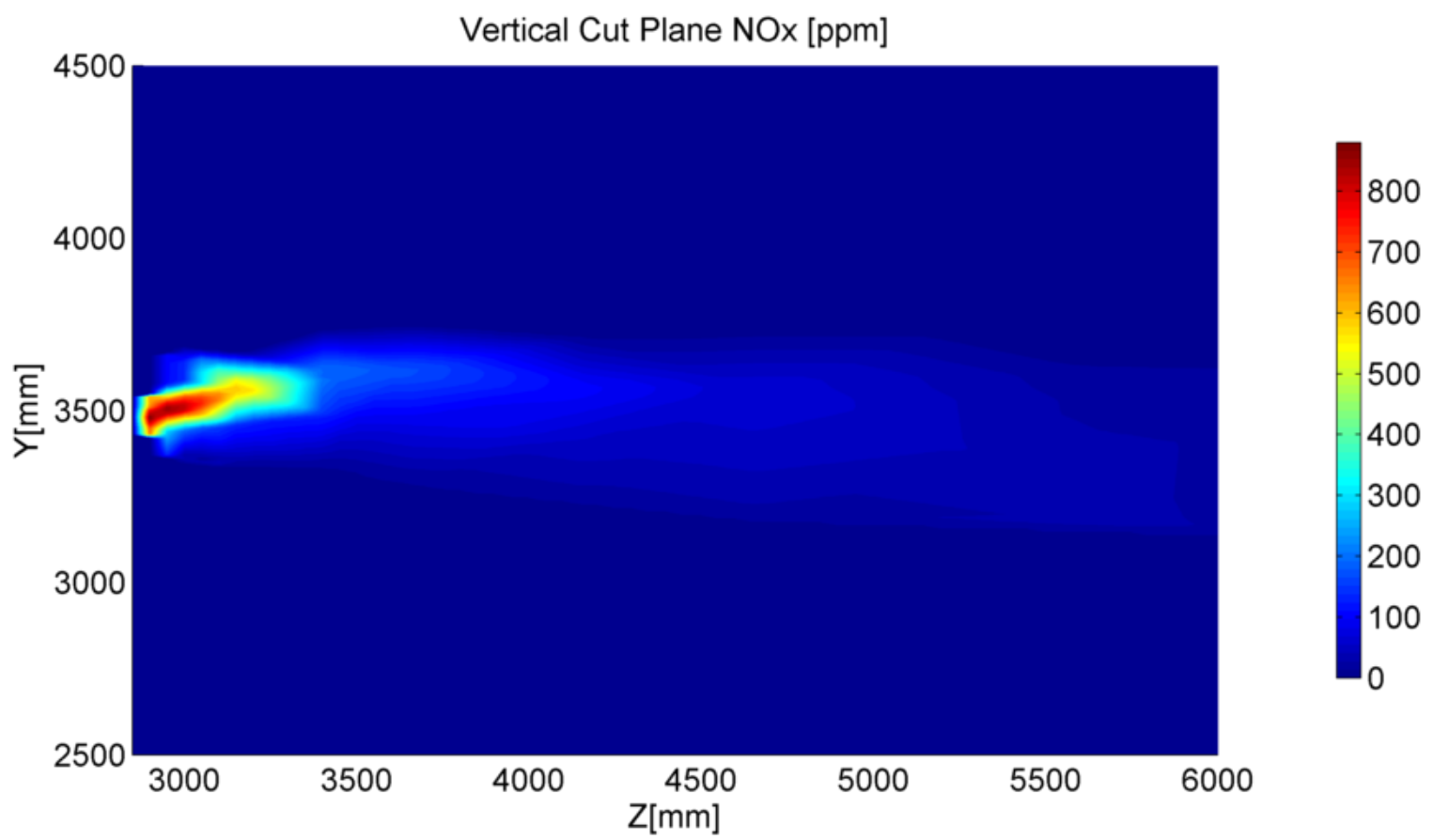

Figure 5-17: Longitudinal vertical contour cut plot of the NOx trace for the Pre-2007 vehicle during the $35 \mathrm{mph}$ test. The plane is passing through the exhaust stack. 


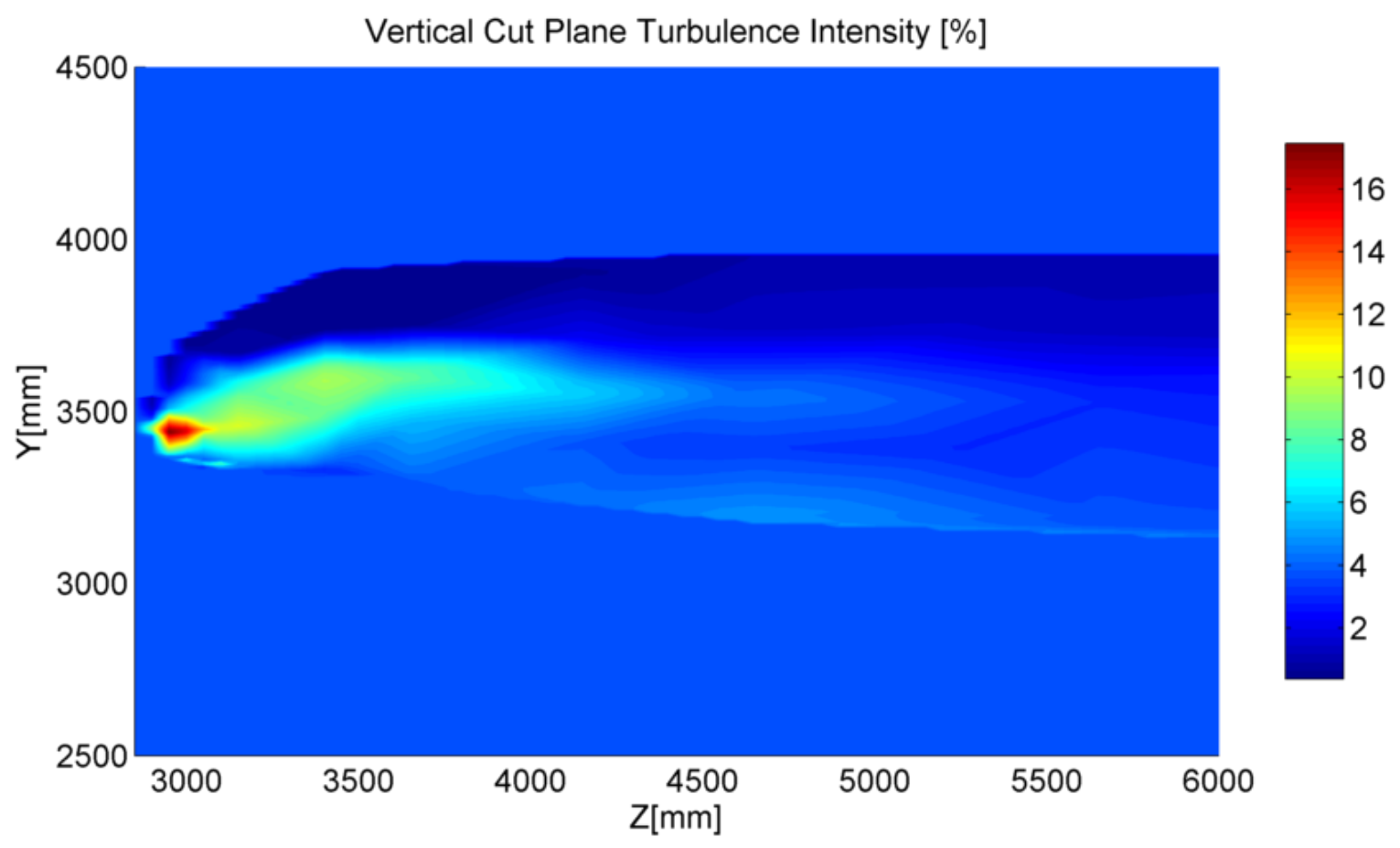

Figure 5-18: Longitudinal vertical contour cut plot of the turbulence intensity trace for the Pre-2007 vehicle during the $35 \mathrm{mph}$ test. The plane is passing through the exhaust stack. 

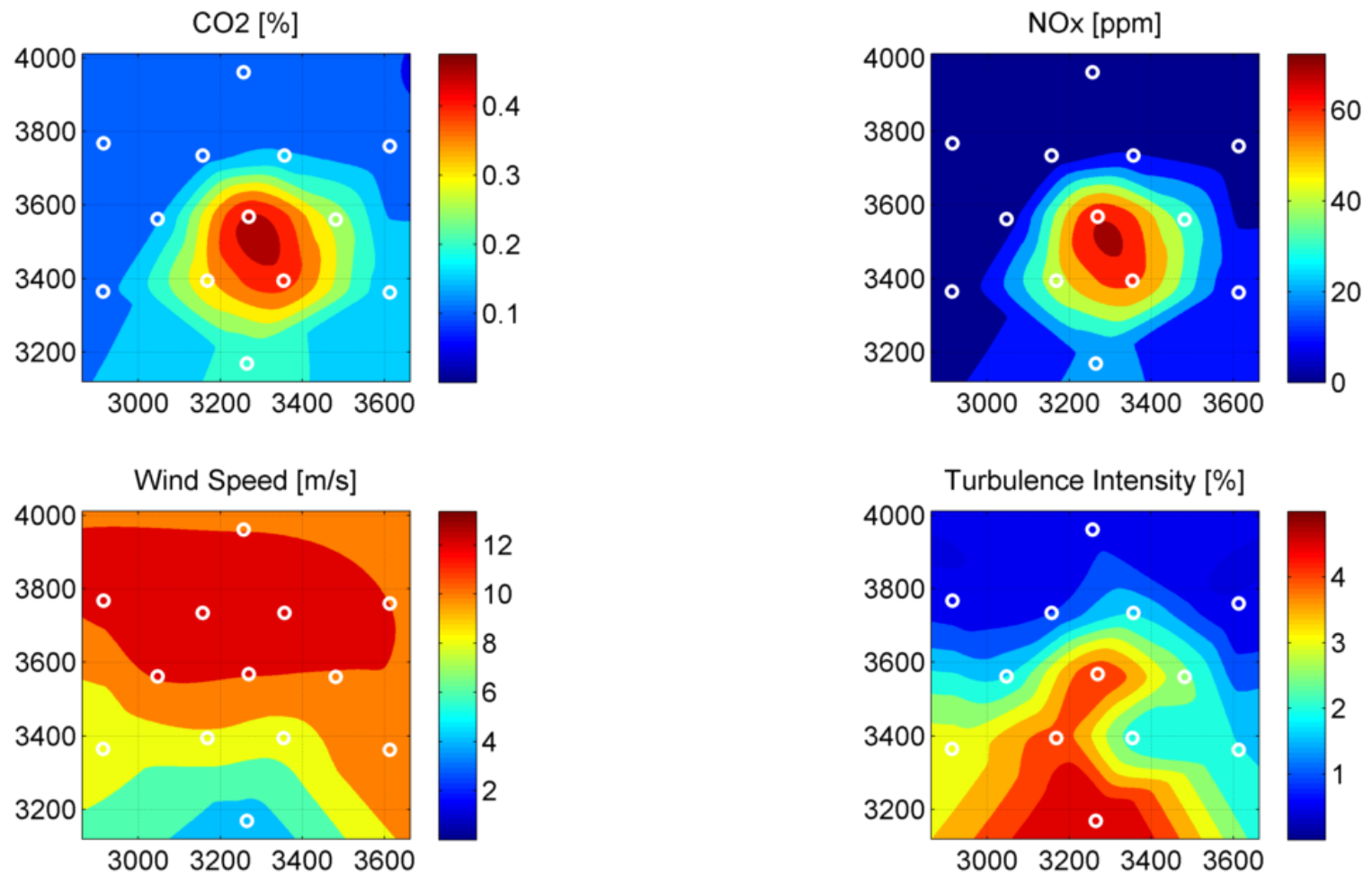

Figure 5-19: Transverse contour cut plot of $\mathrm{CO}_{2}, \mathrm{NOx}$, wind speed, and TI. The plane of this plot is sampling plane 5 , which is $1.315 \mathrm{~m}$ from the emitting point for the $35 \mathrm{mph}$ test of the Pre-2007 vehicle. The white dots represent the defining plane sampling point. 


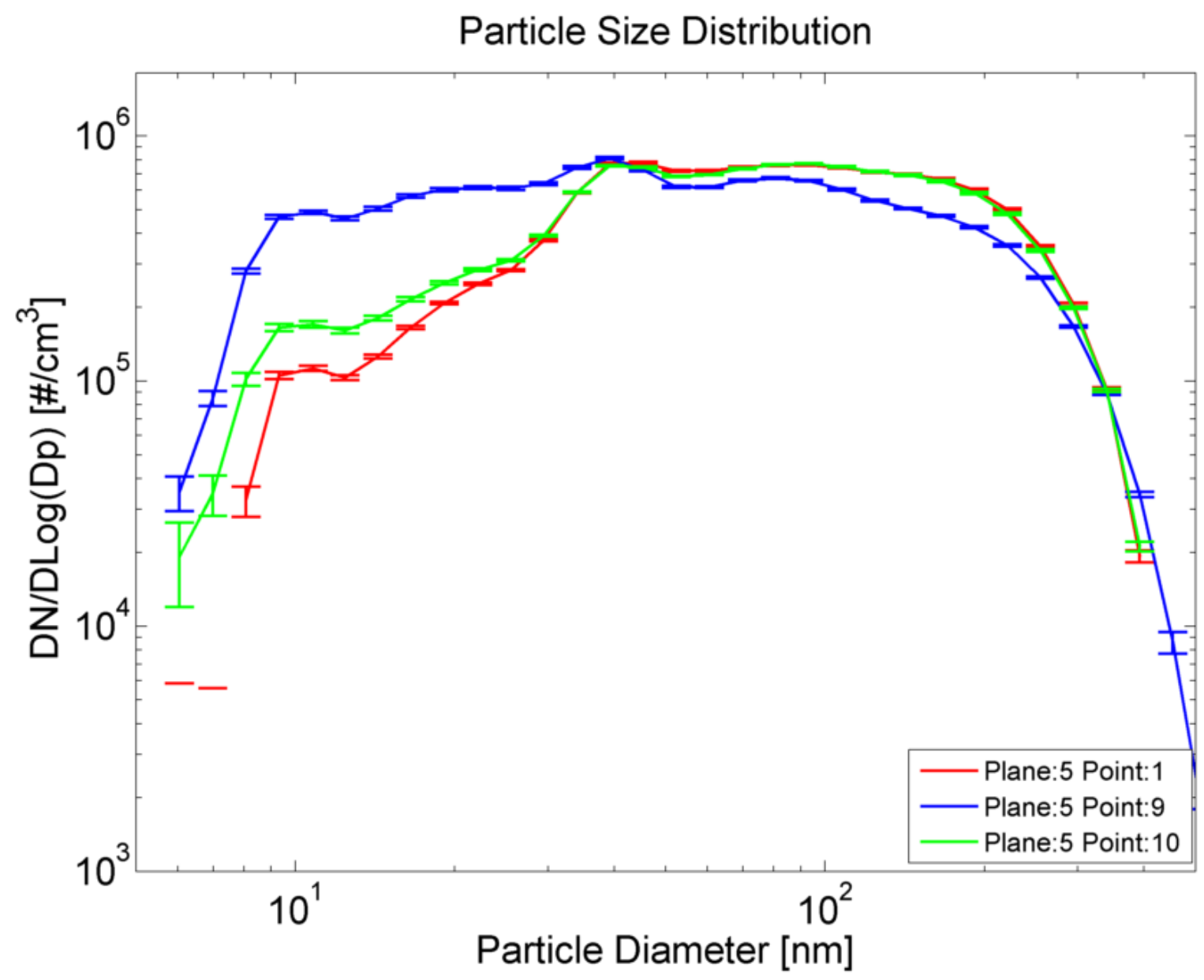

Figure 5-20: Particle size distribution of point 1, point 9, and point 10 of plane $5(1.315 \mathrm{~m}$ downstream) for the $20 \mathrm{mph}$ test of the Pre-2007 vehicle. 


\subsubsection{Post-2007 Vehicle Test Results}

This section shows the results of the Post-2007 vehicle, analyzing the shape and dimension of the plume. Additionally, the goal is to understand how the PM emissions evolve within the plume itself. It needs to be remarked that the DPF of this vehicle was found partially damaged (cracked). Thus, the PM emissions were strongly affected at higher loads.

\subsubsection{Idle Test}

As described previously in the idle test, the vehicle is in idling condition and a wind of $5 \mathrm{mph}$ is generated. This test represents the emissions of the truck in a rest area. Table 58 summarizes the vehicle and dynamometer parameter during the idle test for the Post2007 vehicle.

Table 5-8: Vehicle and Dynamometer Parameter During the Idle Test for the Post-2007 Vehicle.

$\begin{array}{ll}\text { Parameter Value } & \text { Var }\end{array}$

\section{Dynamometer Torque [ft-lb]}

Dynamometer Power [hp]

Dynamometer Speed [mph]

Engine Speed [RPM]

750

\section{Transmission gear}


One of the positive advantages of using the same mock-up cabin inside the wind tunnel, and having similar exhaust flow rates, is to generate aerodynamic fields with negligible differences from one truck to another. This reflects the indication that the differences in plume will be encountered only in the PM particle size distribution and concentration emissions, due to the composition of solid and volatile compounds in the exhaust.

The Post-2007 vehicle is equipped with a DPF and substantially lower solid PM emissions are expected. The reduced solid percentage in the plume leads the volatile compound to have less surface to be absorbed on and thus to nucleate. Analyzing Figure 5-21, the DustTrack trace follows the traces of $\mathrm{CO}_{2}$ and $\mathrm{NO}_{\mathrm{x}}$, with measured values close to the lower limit of the instrument. A relatively unexpected result is identifiable in the total PM count of Figure 5-21, the total PM in the plume is lower than the background PM. This unusual result is related to the DPF and the low load at which the engine is operating. In the same figure, it is evident comparing the total PM count, nucleation mode PM count, and accumulation mode PM count that the balance of PM count is towards the nucleation mode, with a uniform composition across the entire plume.

In Figure 5-22 it is possible to depict this observing the overlapping particle size distribution and concentration for point 1 and 8 of plane 2. The symptoms of a uniform particle size distribution and concentration across the plume in such early stages indicates that the mixing and dilution generated the highest amount of nucleation mode possible. Such information is confirmed by Figure 5-23, where the particle size distribution and concentration for points 1 and 10 of the plane resemble the ones of Figure 5-22. The particle 
size distribution and concentration do not perfectly overlap due to the aging of the PM (coagulation and absorption) and the "poisoning" effect of the background PM, but leave a clear indication as to how the majority of nucleation mode was formed prior to plane 2 . DPF-equipped HDDTs indicate that the nucleation mode is generated by the dilution processes that happen within $30 \mathrm{~cm}$ from the exhaust.
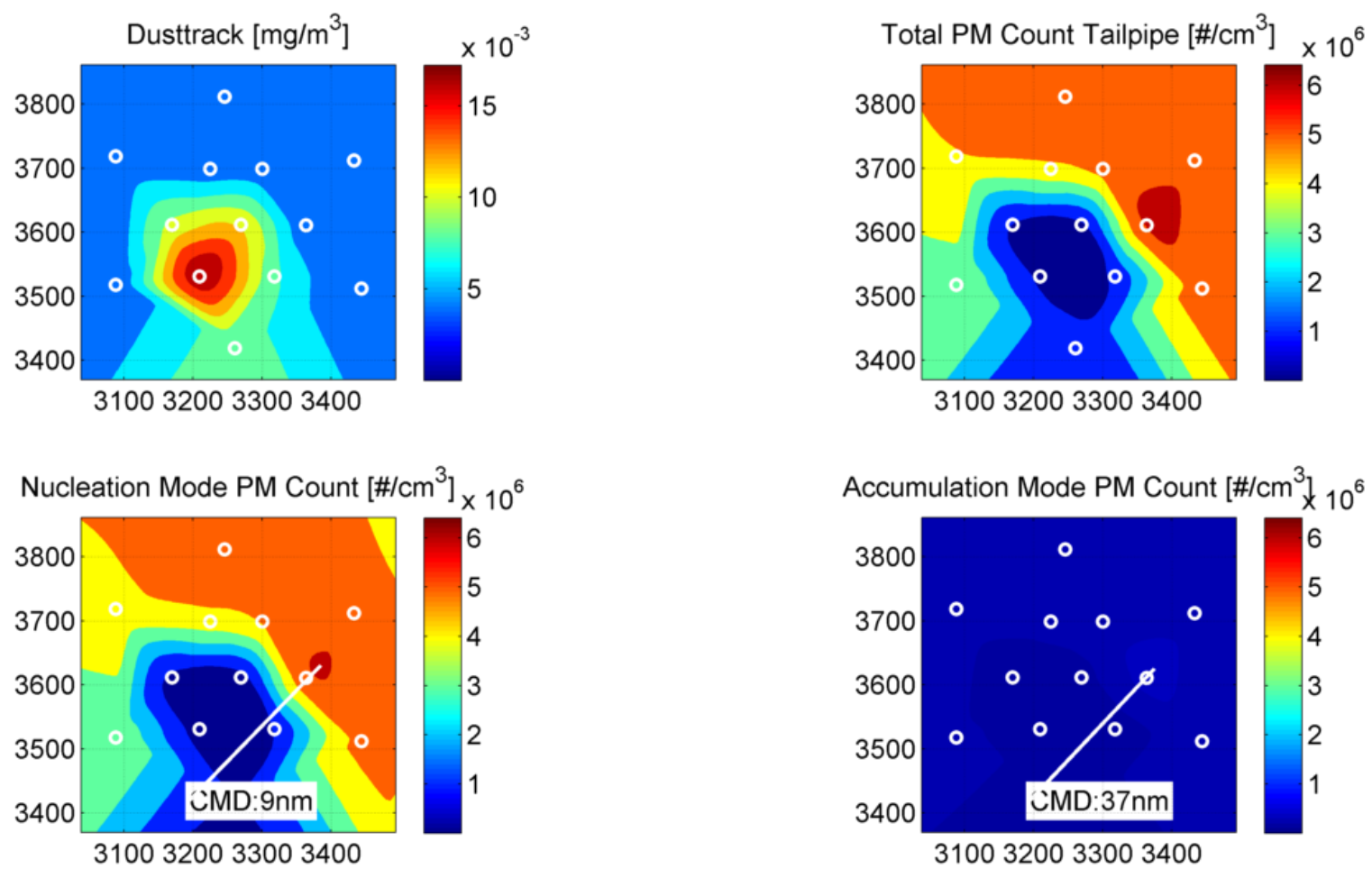

Figure 5-21: Transverse contour cut plot of DustTrack, total PM count tailpipe corrected, nucleation mode PM count, and accumulation mode PM count for the idle test of the Post-2007 vehicle. The plane of this plot is sampling plane 2, which is $0.315 \mathrm{~m}$ from the emitting point. The white dots represent the plane sampling points. 


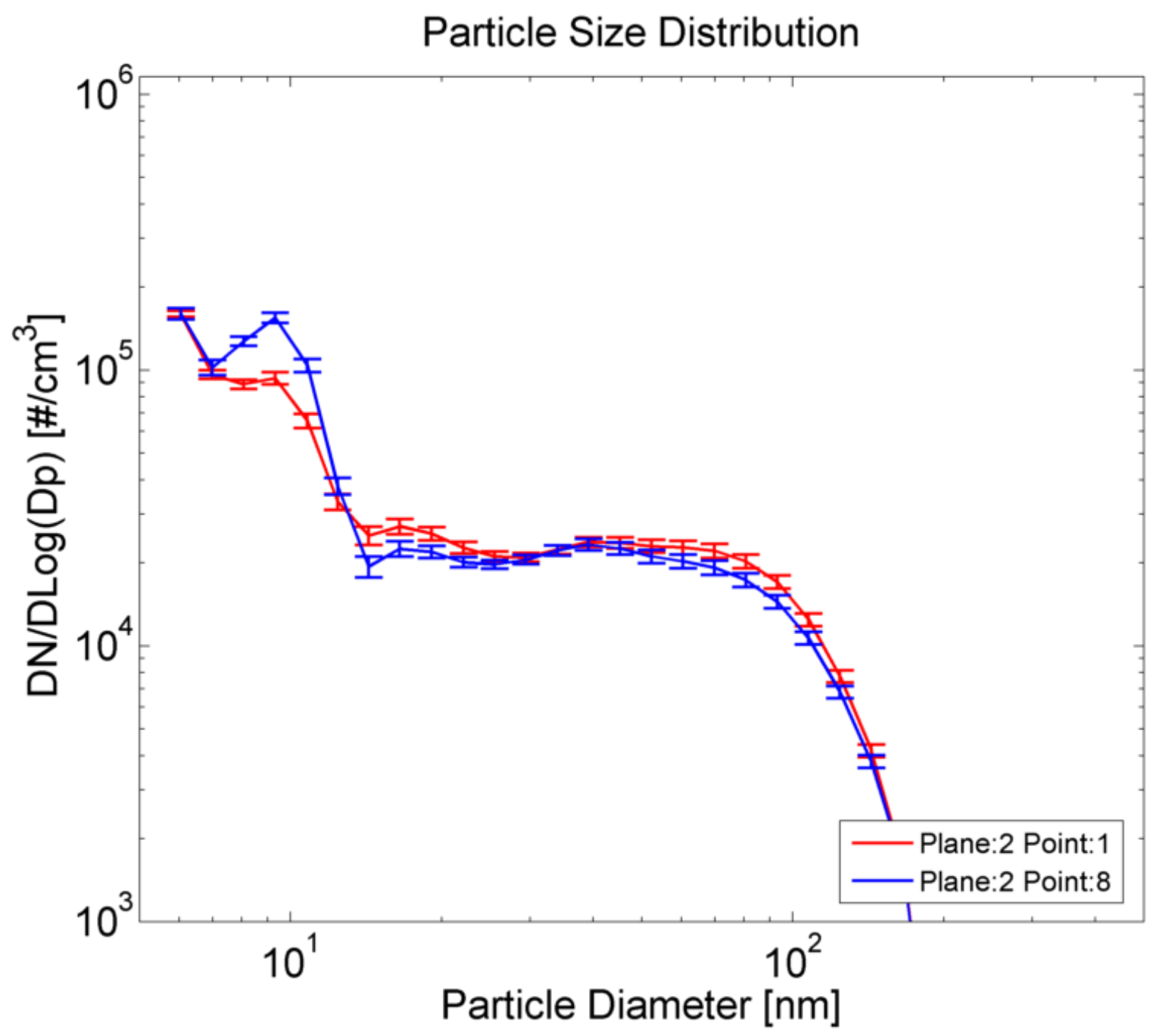

Figure 5-22: Particle size distribution of point 1 , and point 8 of plane $2(0.315 \mathrm{~m}$ downstream) for the idle test of the Post-2007 vehicle. 


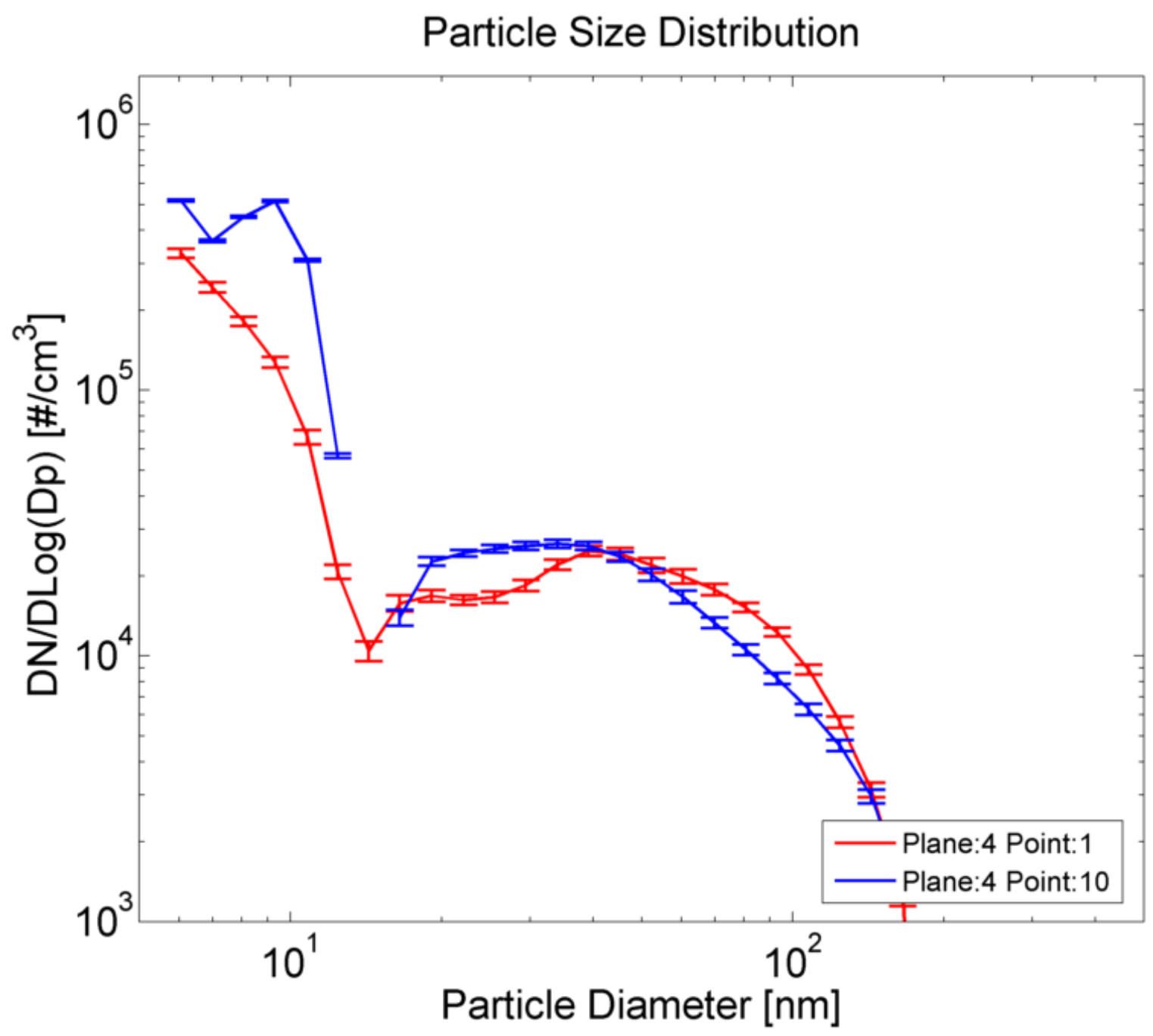

Figure 5-23 Particle size distribution of point 1 , and point 10 of plane $4(0.815 \mathrm{~m}$ downstream) for the idle test of the Post-2007 vehicle. 


\subsubsection{20mph Test}

As described previously in the $20 \mathrm{mph}$ test, the vehicle and wind speed are matched at $20 \mathrm{mph}$. This test represents a HDDT traveling at $20 \mathrm{mph}$ on a flat road in calm air. The road load equation indicates that the total power required at the wheel is $40 \mathrm{hp}$. Table 5-9 summarizes the vehicle and dynamometer parameters during the $20 \mathrm{mph}$ test for the Post2007 vehicle.

Table 5-9: Vehicle and Dynamometer Parameters During the 20mph Test for the Post-2007 Vehicle.

\begin{tabular}{lc}
\hline Parameter & Value \\
\hline Dynamometer Torque [ft-lb] & 300 \\
\hline Dynamometer Power [hp] & 40 \\
\hline Dynamometer Speed [mph] & 20 \\
\hline Engine Speed [RPM] & 1400 \\
\hline Transmission gear & 6 th \\
\hline
\end{tabular}

$\mathrm{CO}_{2}$ and $\mathrm{NO}_{\mathrm{x}}$ traces, plume shape, and dimension analyses are omitted in this paragraph, since aerodynamic fields for this test are similar to the $20 \mathrm{mph}$ test for the Pre2007 vehicle. Thus, concentration will focus more on PM analysis. As it was possible to observe in the idle test of this Post-2007 vehicle, the amount of solid PM is substantially lower than a non-equipped DPF vehicle (Pre-2007). The same behavior is observed also in this test, where the Dust Track indicates very low values of soot and the nucleation mode is the predominant component in the particle count already at a short distance from the 
exhaust stack (see Figure 5-24). Different from the idle test of this vehicle, the PM in the plume is higher than the background air, due in part to higher load the engine is experiencing, but also the cracked DPF allows the escape of part of the soot. Although the soot escape is not enough to match the emissions from the Pre-2007 vehicle.
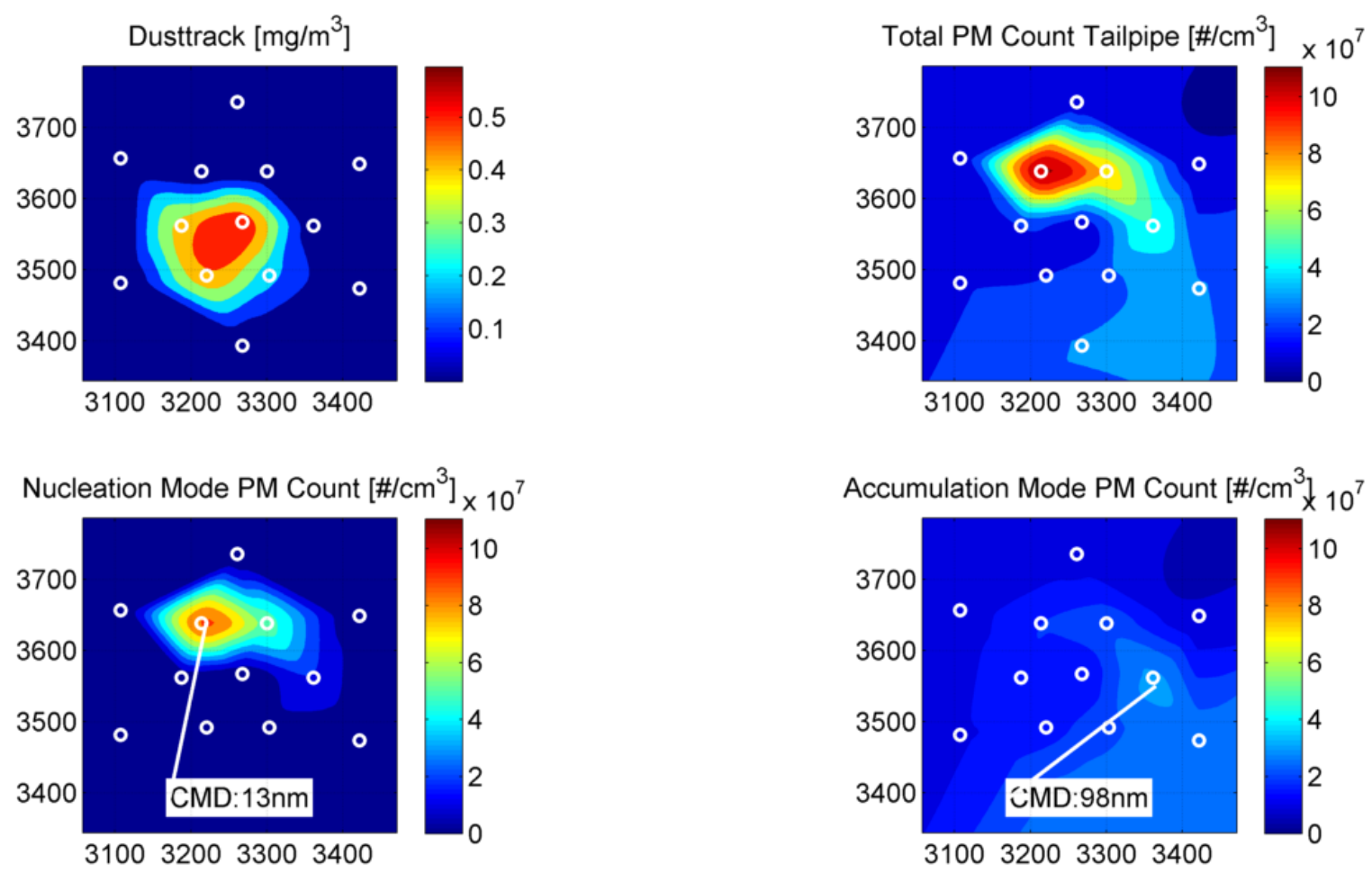

Figure 5-24: Transverse contour cut plot of DustTrack, total PM count tailpipe corrected, nucleation mode PM count, and accumulation mode PM count for the $20 \mathrm{mph}$ test of the Post-2007 vehicle. The plane of this plot is sampling plane 2, which is $0.315 \mathrm{~m}$ from the emitting point. The white dots represent the plane sampling points. 


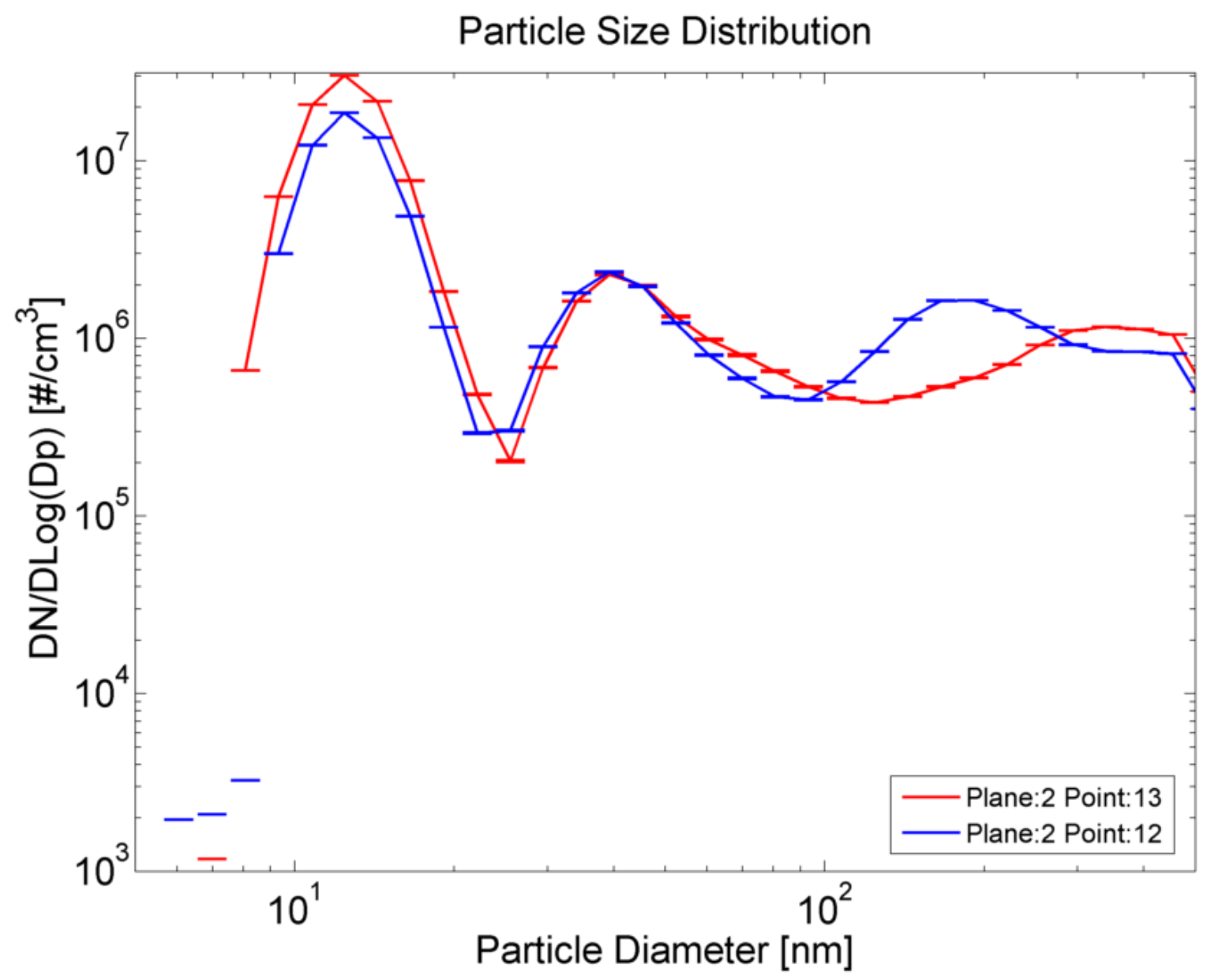

Figure 5-25: Particle size distribution of point 12 , and point 13 of plane $2(0.315 \mathrm{~m}$ downstream) for the 20mph test of the Post-2007 vehicle.

Analyzing the particle size distribution and concentration of points 12 and 13 of plane 2 (peak of total and nucleation mode PM count), it resembles evidence of the dominance of the nucleation mode over the accumulation mode. The accumulation mode appears composed of two non-constant peaks; such results could be an effect of the cracked DPF. The concentration peak in the nucleation mode reaches a value of $2 \times 10^{7}$, the highest value encountered so far. Once again, the nucleation mode appears fully developed across the entire plume at early stages. 
As mentioned earlier, the crack in the DPF was not significant enough to prevent loading of the DPF, and a subsequent active regeneration cycle started halfway through this test. This regeneration event was not complete and lasted only a few minutes. The active regeneration system on this HDDT operates injecting fuel directly into the engineout exhaust via a $7^{\text {th }}$ injector. During the regeneration event (observed between point 8 and point 13 of plane 6), there was an increase of $\mathrm{CO}_{2}$ emission concentrations from $8.37 \%$ to $11.45 \%$, while NOx and $\mathrm{HC}$ emissions were largely unaffected. This increase is reflected in the $\mathrm{CO}_{2}$ plot of Figure 5-26, with a shift of the peak of $\mathrm{CO}_{2}$ from the core of the plume (this time indicated by the NOx trace) towards the points collected during the regeneration event.

The regeneration event resulted in an increase in the PM emissions, particularly the accumulation mode. The increase in total PM emissions is related to the reduced filtration efficiency of the DPF during the regeneration event. The total PM count plot in Figure 527 shows how the plume contour was transformed from a "hill" shape to a "doughnut" shape. The plots of Figure 5-26 and Figure 5-27 still support the notion that TI plays an important role in nucleation mode formation. Analyzing separately the nucleation and accumulation mode, the nucleation mode shows higher particle count during the regeneration event if compared to the rest of the plume for this test, even more pronounced where the $\mathrm{TI}$ is higher, with a CMD of $15 \mathrm{~nm}$. 

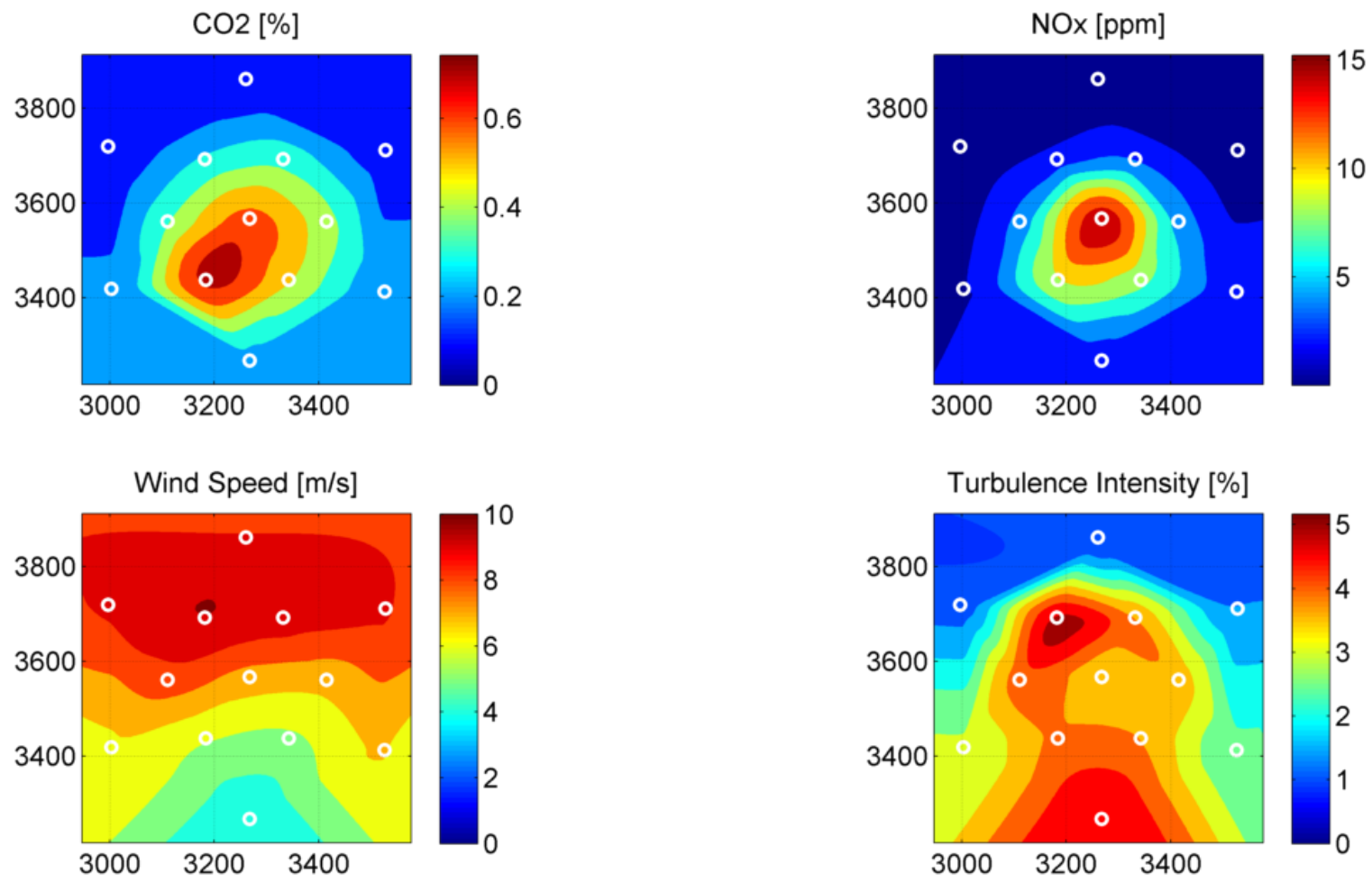

Figure 5-26: Transverse contour cut plot of $\mathrm{CO} 2$, NOx, wind speed, and TI. The plane of this plot is sampling plane 6 , which is $1.815 \mathrm{~m}$ from the emitting point for the 20mph test of the Pre-2007 vehicle. The white dots represent the defining plane sampling points.

The accumulation mode PM count is also greater on the points during the regeneration event. The CMD observed is $150 \mathrm{~nm}$, which is relatively high even for nonequipped DPFs, and the accumulation mode concentration peak (up to $1 \times 10^{7}$ ) is one order of magnitude greater than the nucleation mode, both of which are indicators of the regeneration event. Figure 5-28 presents the particle size distribution and concentration for point 8 and point 12, both during the regeneration event, which represent the points with 
lower TI (point 8) and a point of higher TI (point 12). As expected, both particle size distribution and concentration show similar accumulation modes, while the nucleation mode again shows higher concentration values for the point corresponding to a high TI (point 12).

It is also of interest to note how the regeneration event influences the particle size distribution and concentration. Point 12 of plane 5 (plane sampled prior to the regeneration event and $500 \mathrm{~mm}$ ahead plane 6) and point 12 of plane 6 are both the respective TI peak for their respective plane and share similar NOx concentrations, thus suggesting analogous plume characteristics. Figure 5-29 shows that the regeneration event provokes the formation of a distinct nucleation mode and an increase from one to two orders of magnitude in the concentration of the accumulation mode, with increased CMD.

The nucleation mode in point 12 of plane 5 is not very distinct, mainly because the nucleation mode is driven primarily by unburned $\mathrm{HC}$, and the DOC is quite effective at converting these components. The high exhaust temperature registered at the DOC (in excess of $475^{\circ} \mathrm{C}$ ) suggest the nucleation mode during the regeneration event might be composed of $\mathrm{H}_{2} \mathrm{SO}_{4}$ due to the much higher temperature in the exhaust, as indicated by Rönkkö et al. (2006), even for low fuel-sulfur concentrations. 

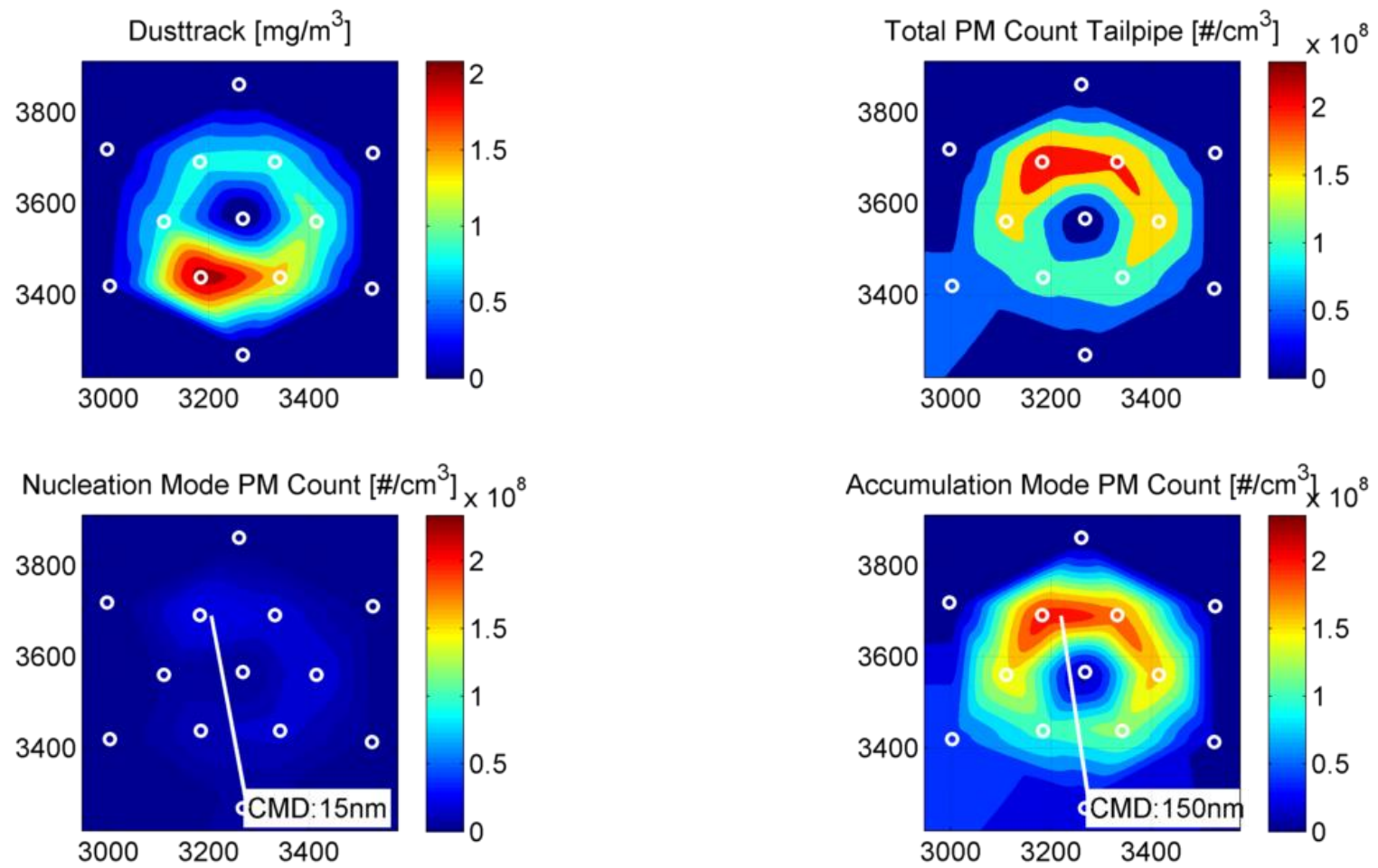

Figure 5-27: Transverse contour cut plot of DustTrack, total PM count tailpipe corrected, nucleation mode PM count, and accumulation mode PM count for the $20 \mathrm{mph}$ test of the Post-2007 vehicle. The plane of this plot is sampling plane 6, which is $1.815 \mathrm{~m}$ from the emitting point. The white dots represent the plane sampling points. 


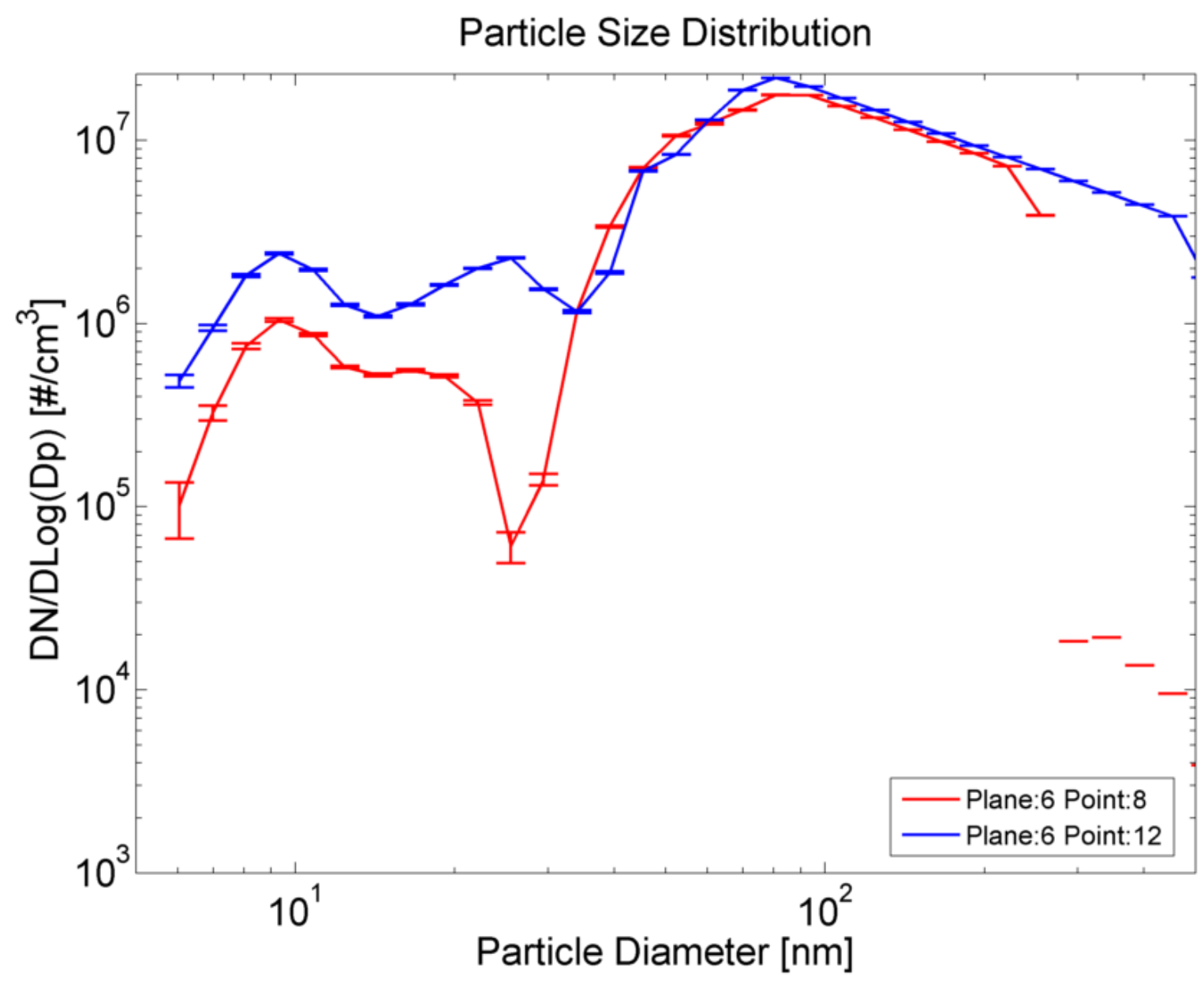

Figure 5-28: Particle size distribution of point 8 , and point 12 of plane $6(1.815 \mathrm{~m}$ downstream) for the 20mph test of the Post-2007 vehicle. 


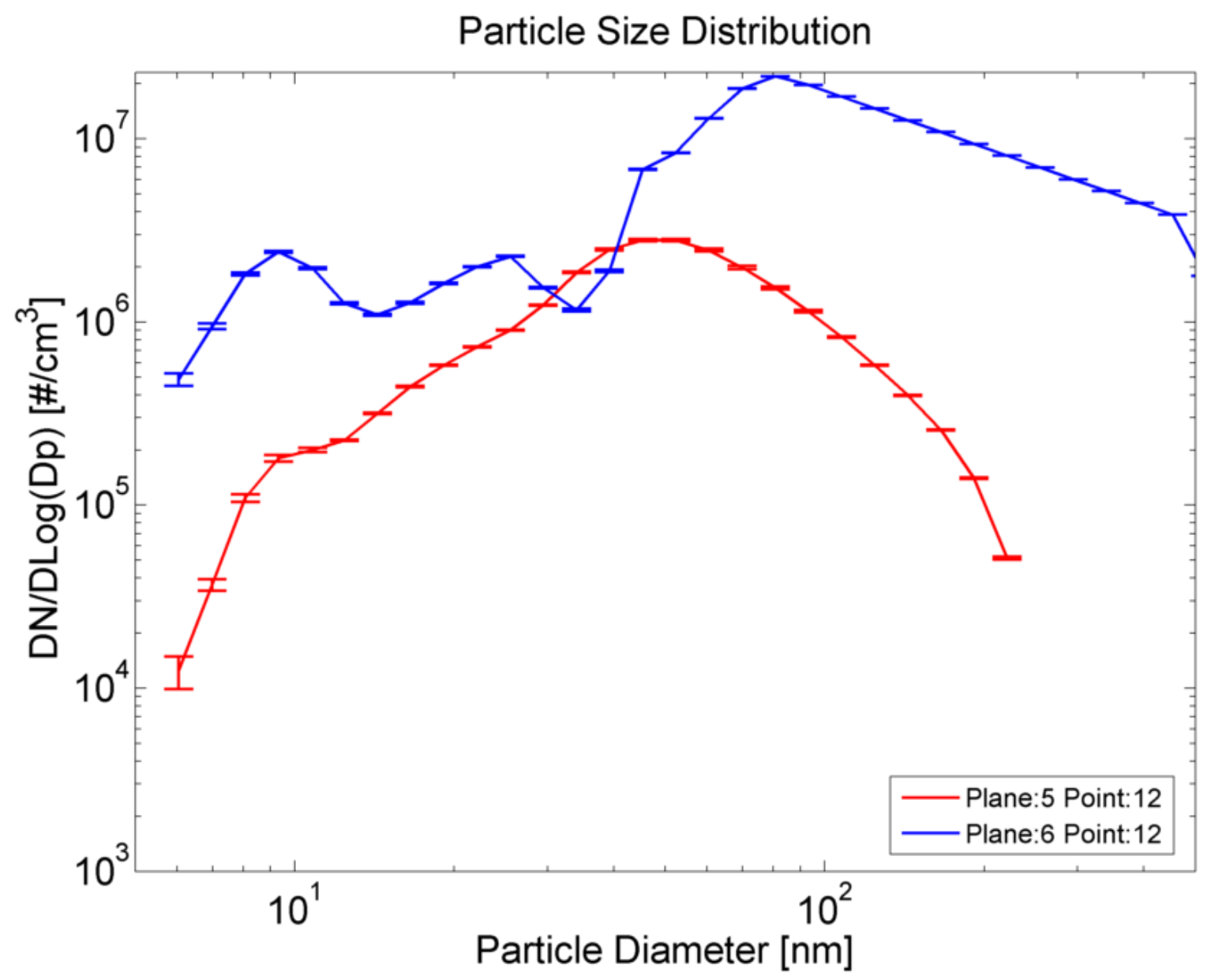

Figure 5-29: Particle size distribution of point 12 of plane 6 (1.815m downstream) and point 12 of plane 5 (1.315m downstream) for the $20 \mathrm{mph}$ test of the Post-2007 vehicle.

\subsubsection{35mph Test}

As described previously in the $35 \mathrm{mph}$ test, the vehicle and wind speed are matched at $35 \mathrm{mph}$. This test represents a HDDT traveling at $35 \mathrm{mph}$ on a flat road in calm air. The road load equation indicates that the total power required at the wheel is $63 \mathrm{hp}$. Table $5-10$ 
summarizes the vehicle and dynamometer parameter during the $20 \mathrm{mph}$ test for the Pre2007 vehicle.

Table 5-10 Vehicle and Dynamometer Parameter During the 35mph Test for the Post-2007 Vehicle.

Parameter Value

Dynamometer Torque [ft-lb] 300

Dynamometer Power [hp] 63

Dynamometer Speed [mph] 35

$\begin{array}{lr}\text { Engine Speed [RPM] } & 1200\end{array}$

$\begin{array}{ll}\text { Transmission gear } & 7 \text { th }\end{array}$

During the warm-up procedure of the instruments and truck prior the beginning of this test, the vehicle started and completed a regeneration event, which lasted for 45 minutes. This event completely regenerated the DPF, while the one experienced during the $20 \mathrm{mph}$ test was only a short, unsuccessful attempt at regeneration. The regeneration event removes PM accumulated on top of the substrate, the so-called "cake layer" (Kittlelson, 1998). This reflects a substantial reduction of filtering efficiency, which ultimately affects the PM emissions in the plume. The reduction in filtering efficiency leads to higher emission in soot and solid compounds, which tends to absorb the volatile compounds and inhibit the formation and growth of the nucleation mode. 

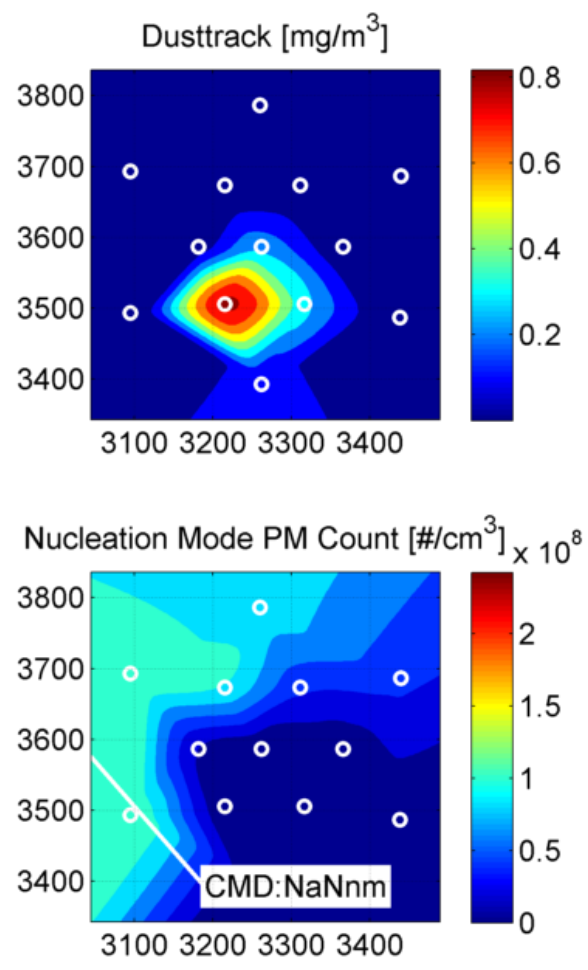
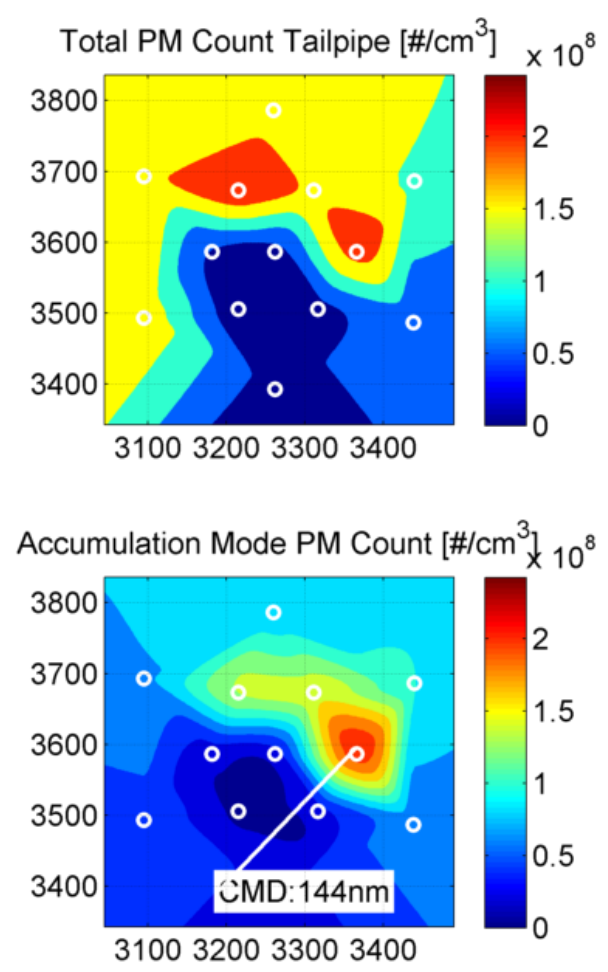

Figure 5-30: Transverse contour cut plot of DustTrack, total PM count tailpipe corrected, nucleation mode PM count, and accumulation mode PM count for the $35 \mathrm{mph}$ test of the Post-2007 vehicle. The plane of this plot is sampling plane 2, which is $0.315 \mathrm{~m}$ from the emitting point. The white dots represent the plane sampling points.

The complex interaction of TI and a newly regenerated DPF did lead to PM emission patterns of non-trivial identification. As noted earlier, plume regions with higher TI led to dominant nucleation mode, especially for DPF-equipped HDDTs, even if damaged. But, a freshly regenerated DPF produces high amounts of solid compounds which inhibit nucleation mode (see Figure 5-30). The tradeoff for this two driving force in 
plane $2(0.315 \mathrm{~m}$ behind the emitting point $)$ allows the plume for an almost constant particle size distribution and concentration across the plume itself (see Figure 5-31).

\section{Particle Size Distribution}

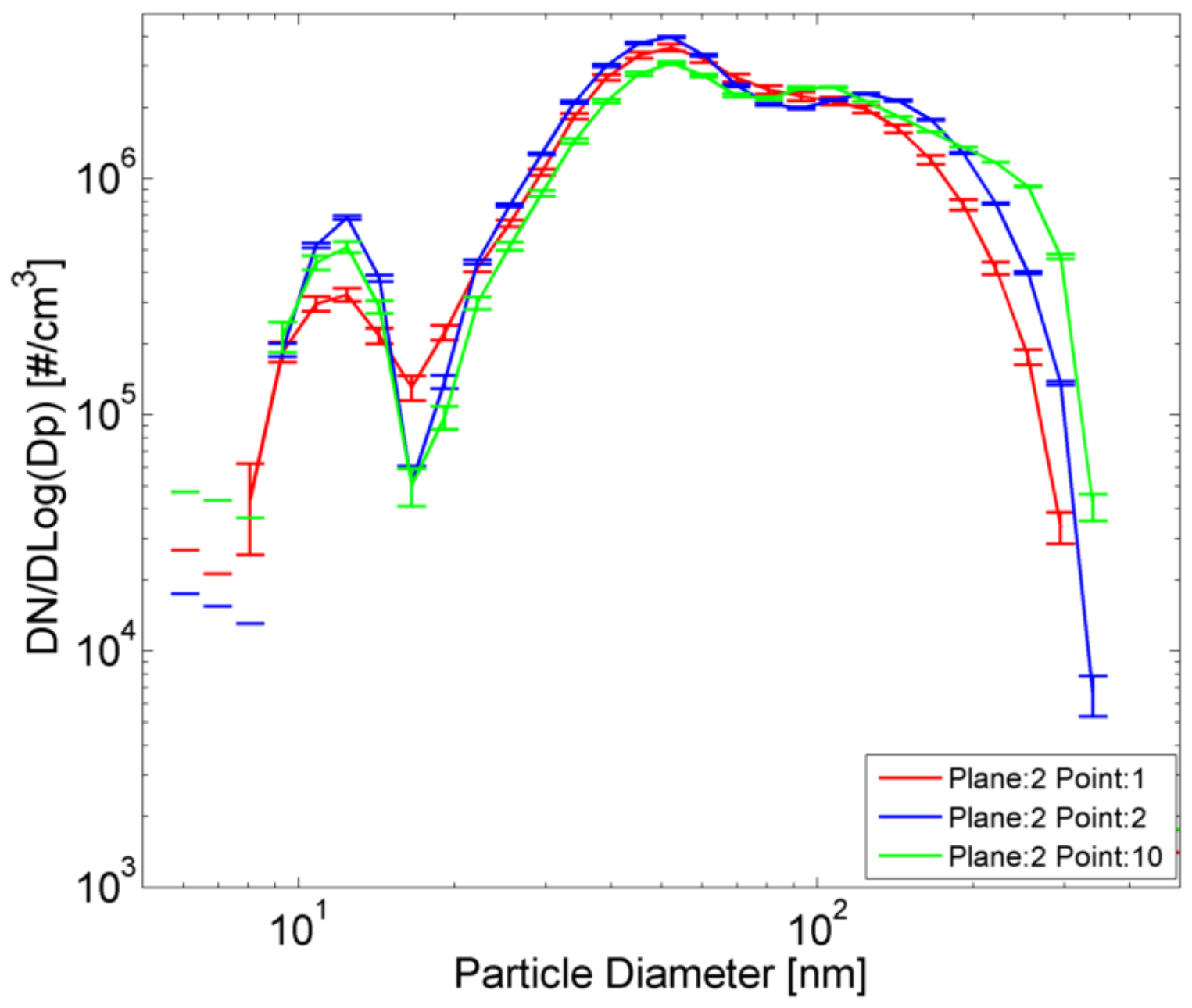

Figure 5-31: Particle size distribution of point 1, point 2, and point 10 of plane $2(0.315 \mathrm{~m}$ downstream) for the $20 \mathrm{mph}$ test of the Pre-2007 vehicle.

A double mode particle size distribution and concentration for point 1 , point 2 , and point 10 is visible, with a remarkably high accumulation mode (similar to non-equipped 
DPF HDDTs) with a concentration peak of up to $4 \times 10^{6}$ (see Figure 5-31).
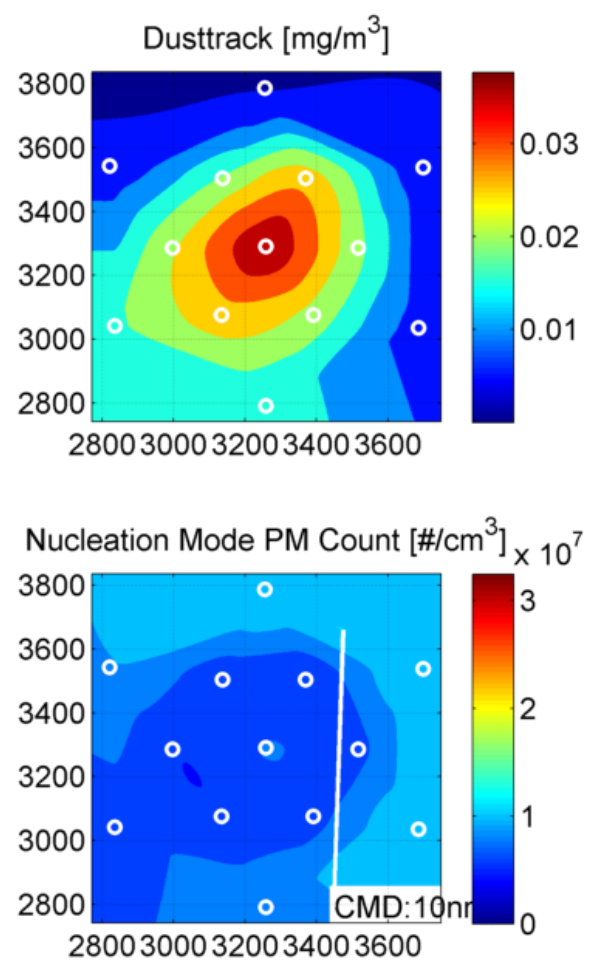
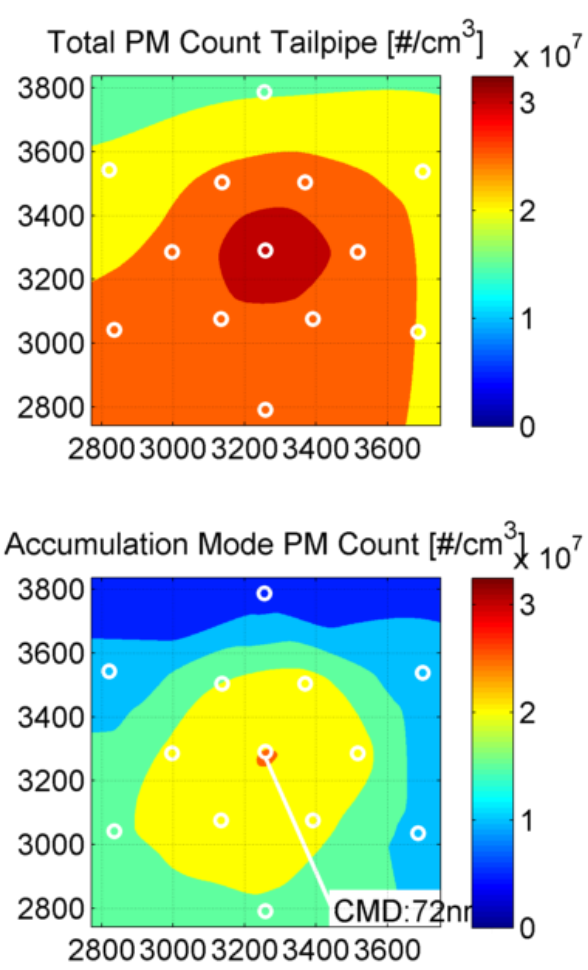

Figure 5-32: Transverse contour cut plot of DustTrack, total PM count tailpipe corrected, nucleation mode PM count, and accumulation mode PM count for the $35 \mathrm{mph}$ test of the Post-2007 vehicle. The plane of this plot is sampling plane 7, which is $2.815 \mathrm{~m}$ from the emitting point. The white dots represent the plane sampling points.

Due to the homogenous composition of the plume with a predominant accumulation mode, it is not expected to observe any major changes in the particle size distribution and concentration for planes further downstream. In plane 7 (2.815m from the emitting point), 
analyzing the plot for total PM count, accumulation mode PM count, and nucleation mode PM count of Figure 5-32, still the accumulation mode is dominant with a CMD of $72 \mathrm{~nm}$.

\section{Particle Size Distribution}

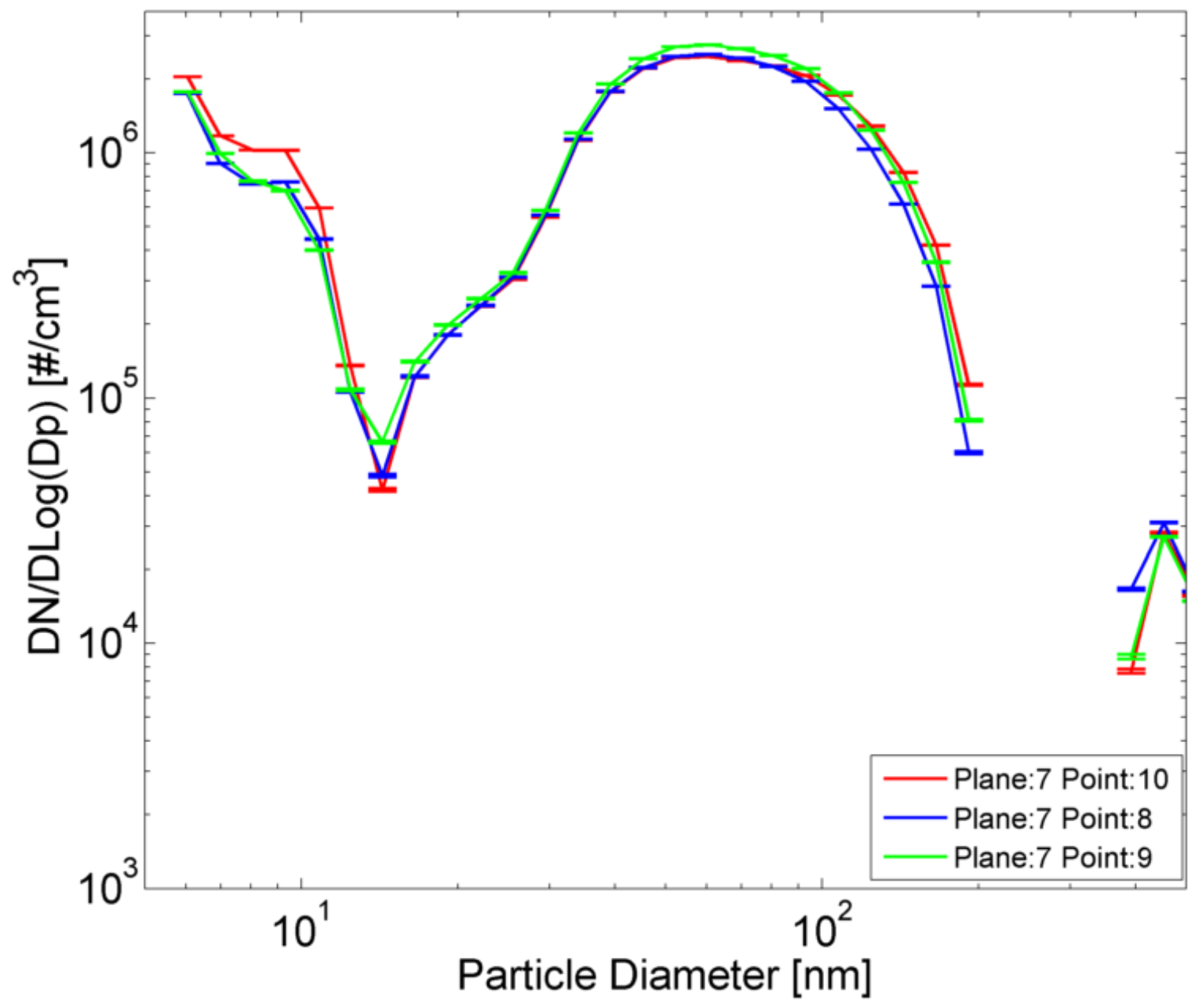

Figure 5-33: Particle size distribution of point 8, point 9, and point 10 of plane $7(2.815 \mathrm{~m}$ downstream) for the $35 \mathrm{mph}$ test of the Post-2007 vehicle.

Particle size distribution across of several points in the plume at plane 7 confirms the homogeneity of PM composition of the plume itself (see Figure 5-33). The 
accumulation mode has slightly changed shape, but not the concentration peak (most likely a coagulation phenomenon).

\subsubsection{Post-2010 Vehicle Test Vehicle}

This section shows the results of the Post-2010 vehicle, analyzing the shape and dimension of the plume. Additionally, the goal is to understand how the PM emissions evolve within the plume itself. Due to the low $\mathrm{NO}_{\mathrm{x}}$ emissions produced by this vehicle, the DR is calculated upon the $\mathrm{CO}_{2}$ emissions.

\subsubsection{Idle Test}

As described previously in the idle test, the vehicle is in idling condition and a wind of $5 \mathrm{mph}$ is generated. This test represents the emissions of a truck in a rest area. Table 511 summarizes the vehicle and dynamometer parameters during the idle test for the Post2010 vehicle. Emissions measured for the idle test from the Post-2010 trucks were significantly lower than those measured from the Pre-2007 and Post-2007 trucks for the $\mathrm{PM}, \mathrm{CO}, \mathrm{CO}_{2}$, and $\mathrm{HC}$; but not for $\mathrm{NO}_{\mathrm{x}}$. Although this truck was outfitted with SCR for NOx control, at plane $2(0.315 \mathrm{~m}$ after the exhaust stack $)$ NOx results indicated that the exhaust temperatures were likely insufficient to provide for high $\mathrm{NO}_{\mathrm{x}}$ conversion rates, as $\mathrm{NO}_{\mathrm{x}}$ was still at appreciable levels as shown by Figure 5-34. 
Table 5-11: Vehicle and Dynamometer Parameter During the Idle Test for the Post-2010 Vehicle.

Parameter Value

Dynamometer Torque [ft-lb]

Dynamometer Power [hp]

Dynamometer Speed [mph]

$\begin{array}{lr}\text { Engine Speed [RPM] } & 800\end{array}$

Transmission gear

Figure 5-34 indicates that the $\mathrm{CO}_{2}$ and $\mathrm{NO}_{\mathrm{x}}$ measurements provide for similar contour plots, affording good indication of position, shape, and dimensions of the plume. As was the case for the other trucks, the TI contour still presents an asymmetrical shape, with higher TI in the lower region of the plume and higher speed in the upper. The DPF of this vehicle appeared to be functioning properly, forecasting PM formation/maturation with a different pattern if compared to the previous trucks. The DustTrack trace in Figure 5-35 shows an extremely low level of solid PM, lower than the idle test of the Post-2007 vehicle. The total PM count, nucleation mode PM count, and accumulation mode PM count plots of Figure 5-35 indicate a plume with lower PM content than the background air. Once again, the explanation is related to the low load applied to the engine and the integrity of the DPF. Particle size distribution and concentration of point 1 and point 8 of plane $2(0.315 \mathrm{~m}$ downstream $)$ have values below the lower threshold of the EEPS ${ }^{\circledR}$, and 
should be essentially disregarded. This combination of truck and test shows almost undetectable PM, no further plots are shown.
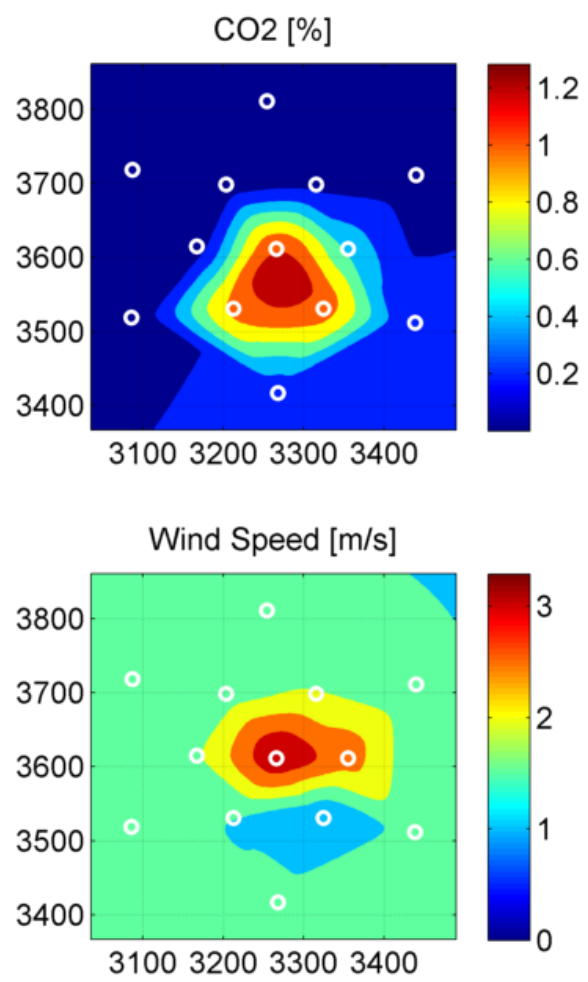
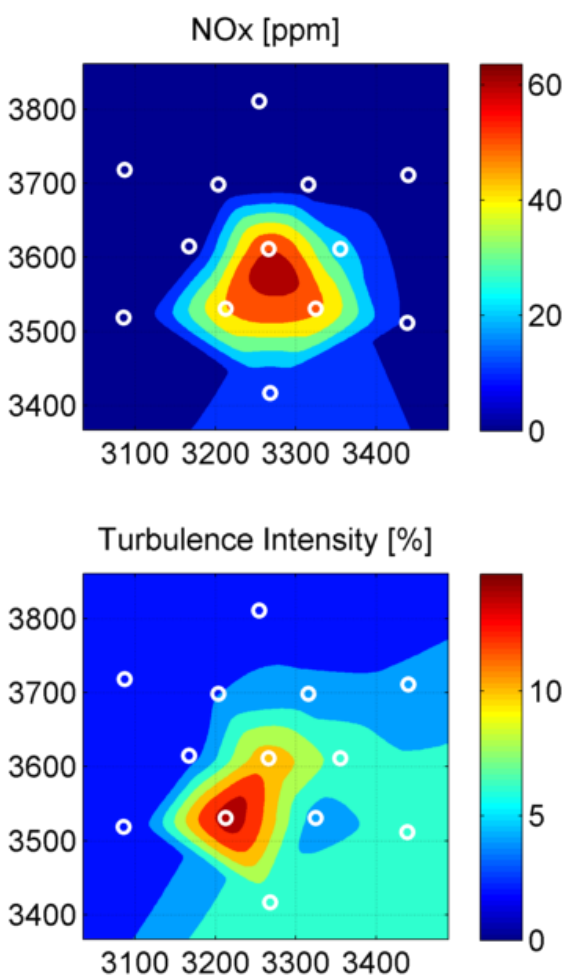

Figure 5-34: Transverse contour cut plot of $\mathrm{CO}_{2}, \mathrm{NOx}$, wind speed, and TI. The plane of this plot is sampling plane 2 , which is $0.315 \mathrm{~m}$ from the emitting point for the idle test of the Post-2010 vehicle. The white dots represent the defining plane sampling points. 

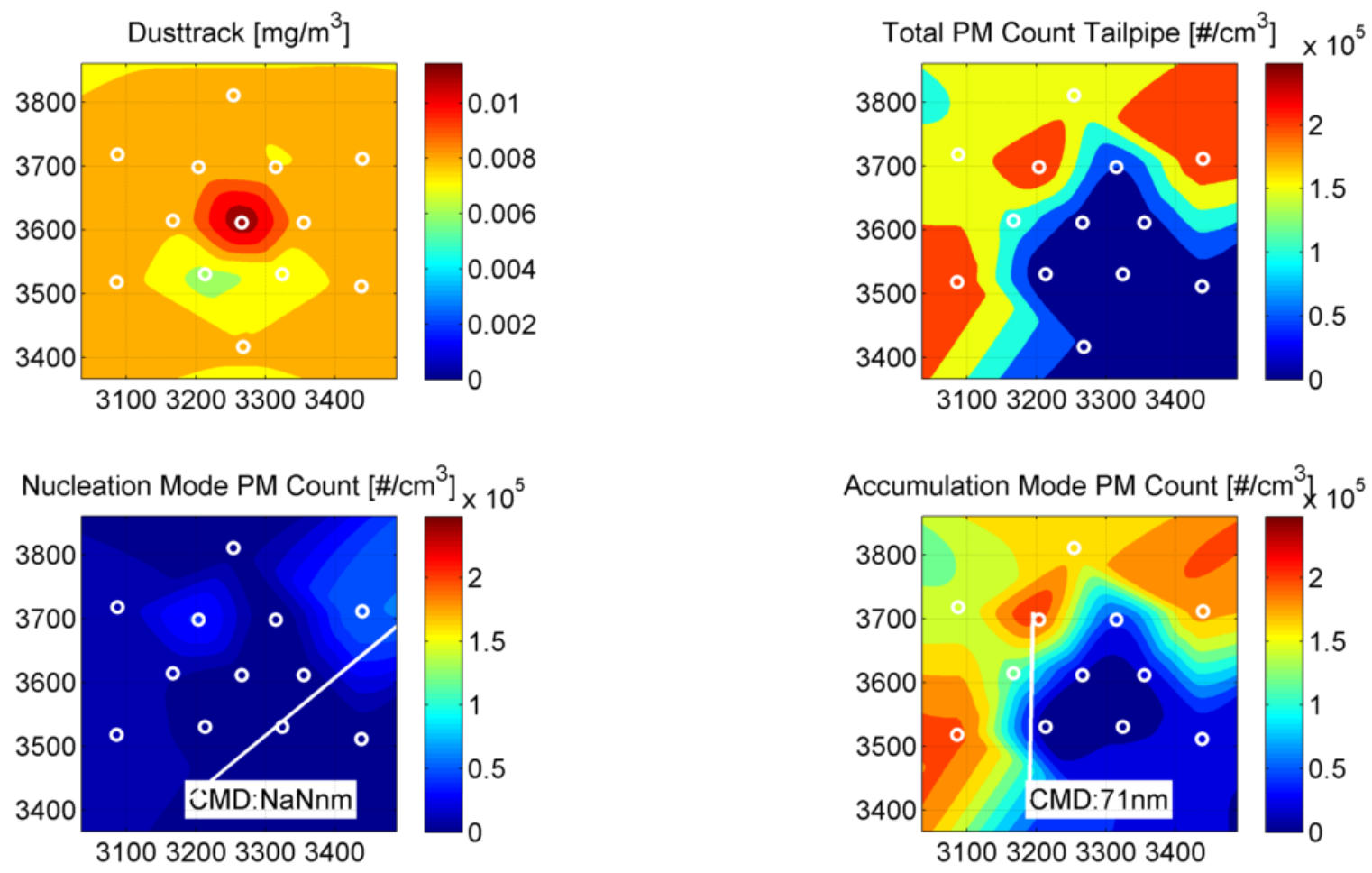

Figure 5-35: Transverse contour cut plot of DustTrack, total PM count tailpipe corrected, nucleation mode PM count, and accumulation mode PM count for the idle test of the Post-2010 vehicle. The plane of this plot is sampling plane 2, which is $0.315 \mathrm{~m}$ from the emitting point. The white dots represent the plane sampling points. 


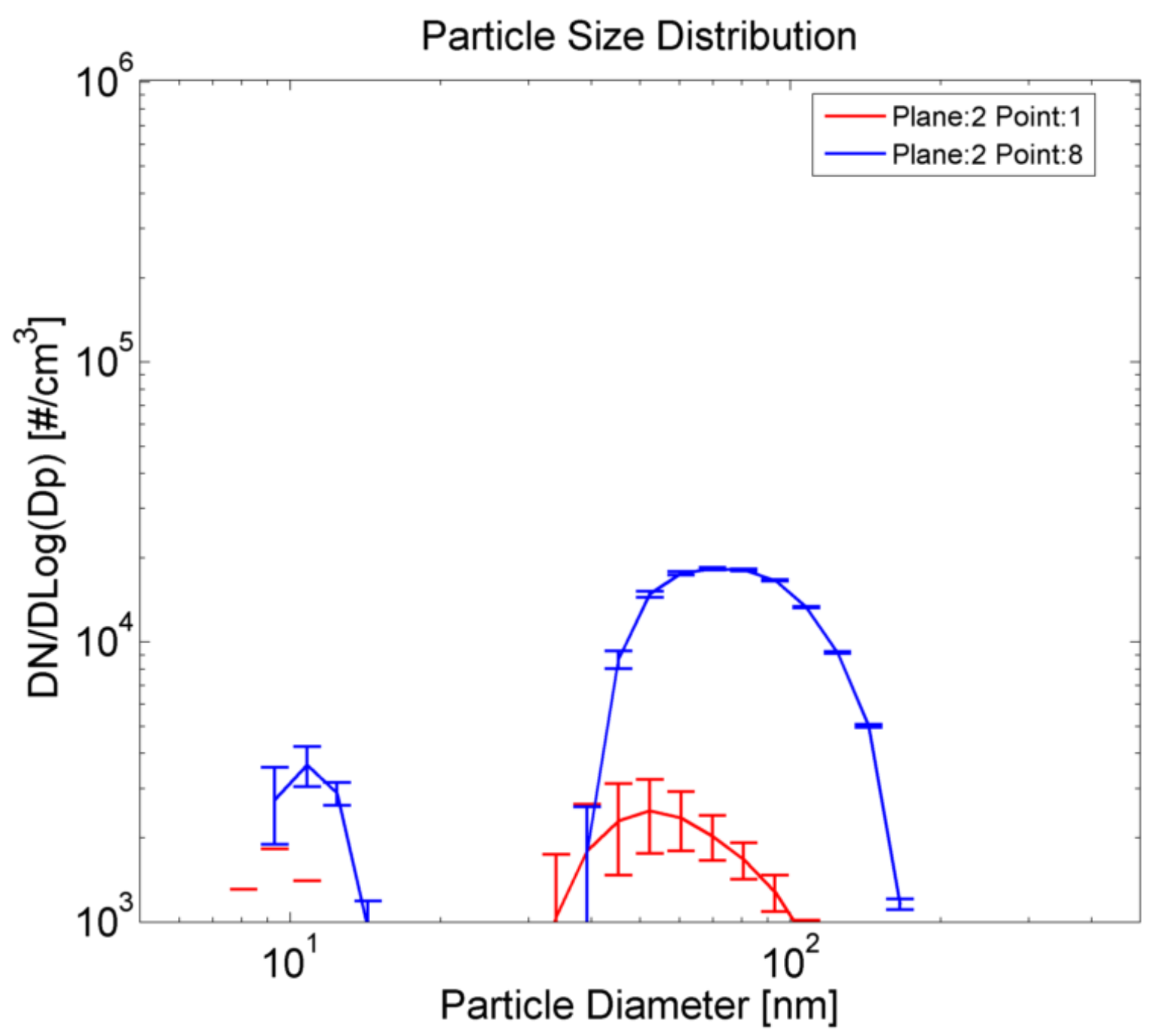

Figure 5-36: Particle size distribution of point 1 , and point 8 of plane $2(0.315 \mathrm{~m}$ downstream) for the idle test of the Post-2010 vehicle. 


\subsubsection{2. $20 \mathrm{mph}$ Test}

As described previously in the $20 \mathrm{mph}$ test, the vehicle and wind speed are matched at $20 \mathrm{mph}$. This test represents a HDDT traveling at $20 \mathrm{mph}$ on a flat road in calm air. The road load equation indicates that the total power required at the wheel is $40 \mathrm{hp}$. Table 5-12 summarizes the vehicle and dynamometer parameters during the $20 \mathrm{mph}$ test for the Post2010 vehicle.

Table 5-12: Vehicle and Dynamometer Parameters During the 20mph Test for the Post2010 Vehicle.

\begin{tabular}{lc}
\hline Parameter & Value \\
\hline Dynamometer Torque [ft-lb] & 300 \\
\hline Dynamometer Power [hp] & 40 \\
\hline Dynamometer Speed [mph] & 20 \\
\hline Engine Speed [RPM] & 1550 \\
\hline Transmission gear & 6 th \\
\hline
\end{tabular}

In the same way of the idle test, where the plume resulted with lower PM content than the background air, no traceable PM is identified in this test. Figure 5-37 provides PM plots that confirm the last statement. 

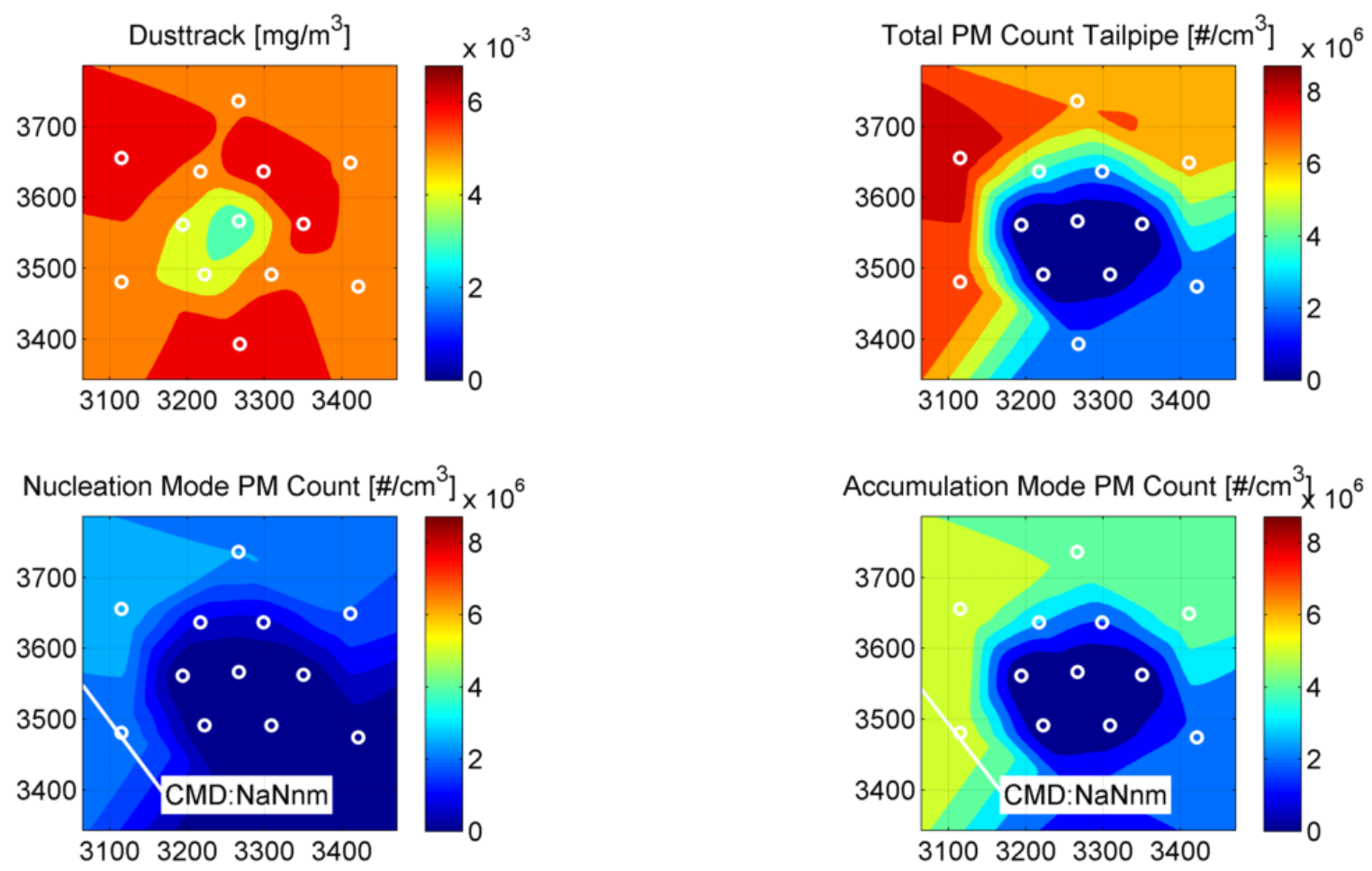

Figure 5-37: Transverse contour cut plot of DustTrack, total PM count tailpipe corrected, nucleation mode PM count, and accumulation mode PM count for the $20 \mathrm{mph}$ test of the Post-2010 vehicle. The plane of this plot is sampling plane 2, which is $0.315 \mathrm{~m}$ from the emitting point. The white dots represent the plane sampling points. 


\subsubsection{35mph Test}

As described previously in the $35 \mathrm{mph}$ test, the vehicle and wind speed are matched at $35 \mathrm{mph}$. This test represents a HDDT traveling at $35 \mathrm{mph}$ on a flat road in calm air. The road load equation indicates that the total power required at the wheel is $63 \mathrm{hp}$. Table $5-13$ summarizes the vehicle and dynamometer parameters during the $35 \mathrm{mph}$ test for the Pre2010 vehicle.

Table 5-13: Vehicle and Dynamometer Parameters During the 35mph Test for the Post2010 Vehicle.

\begin{tabular}{lc}
\hline Parameter & Value \\
\hline Dynamometer Torque [ft-lb] & 300 \\
\hline Dynamometer Power [hp] & 69 \\
\hline Dynamometer Speed [mph] & 35 \\
\hline Engine Speed [RPM] & 1400 \\
\hline Transmission gear & 8 th \\
\hline
\end{tabular}

The increase in load for this test is not sufficient enough to produce substantial PM emissions. Figure 5-38 once again demonstrates how the background PM contains more PM than the plume. 

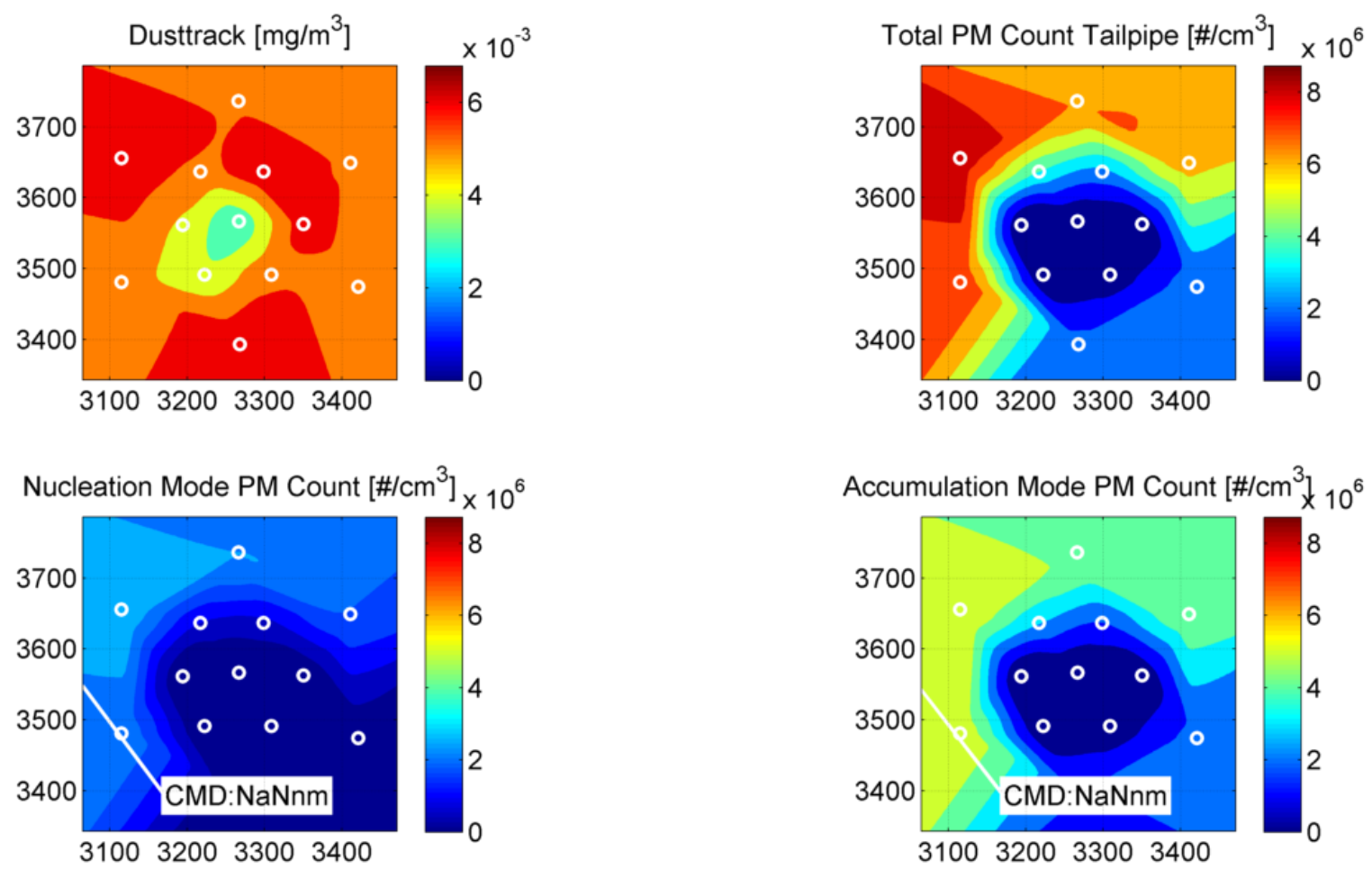

Figure 5-38: Transverse contour cut plot of DustTrack, total PM count tailpipe corrected, nucleation mode PM count, and accumulation mode PM count for the $35 \mathrm{mph}$ test of the Post-2010 vehicle. The plane of this plot is sampling plane 2, which is $0.315 \mathrm{~m}$ from the emitting point. The white dots represent the plane sampling points. 


\subsection{Comparison of PM Plume Measurements with STANDaRdized Laboratory TECHNIQUES.}

In this section, the PM emissions sampled from the plume are compared with measurements performed simultaneously from the raw exhaust with a DDS. The key factor in the comparison is to find in the 130 sampled plume points, the ones with a DR similar to the DDS. It is predicted that more than one point can be found in the plume, providing further information on how the dilution history can affect the particle size distribution and concentration of the PM.

\subsubsection{Pre-2007 Vehicle Comparison Results}

This section shows the PM emissions comparison of the Pre-2007 vehicle for plume and raw exhaust measurements of PM.

\subsubsection{Idle Test}

The setup chosen for the DDS system is summarized in Table 5-14. The total DR for this setup is 6, and the three identified points in the plume present a DR that falls within a range of $\pm 2.5 \%$. Analyzing the particle size distribution for the plume points in Figure 539 , it is evident that they all present an overlapping trace. This indicates that no coagulation or PM maturation effects are observed in the plume for the selected points. The comparison of the DDS measurement with DMS indicates a similar accumulation mode, although the shift present is not fully understood. One possible explanation is the interaction with background air, which may have triggered coagulation towards larger particle diameters. The most notable difference is depicted in the nucleation mode, where the DDS generates a nucleation mode one order of magnitude higher in concentration. This is indicative of the 
combination of the raw exhaust measurements with DDS as it provokes higher nucleation mode concentration than real-world conditions with similar DR. The increase in nucleation mode may be the symptom of higher dilution rate. The shift in transition zone between accumulation and nucleation mode could also be explained by the interaction with background air.

Table 5-14: DDS Parameters Used During the PM Particle Size Distribution and Concentration Sampling from Raw Exhaust for the Pre-2007 Vehicle in the Idle Test.

Parameter Value

Primary Dilution Air Temp [C] 200

Primary Dilution Air Pressure [psi] 35

Secondary Dilution Air Temp [C] 40

Secondary Dilution Air Pressure [psi] 75

Total DR 6

PM spectrometer DMS 


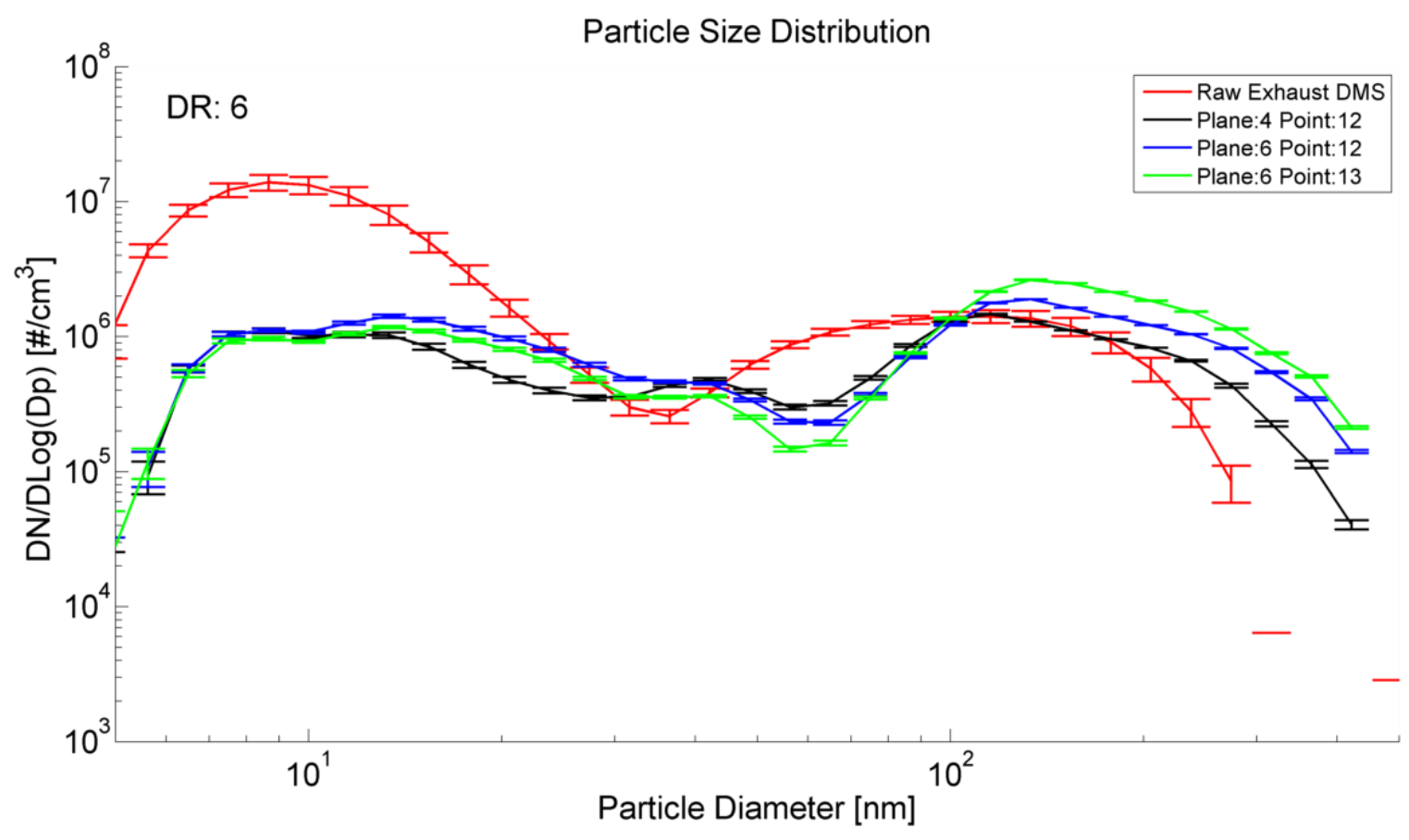

Figure 5-39: PM particle size distribution and concentration comparison between the DMS DDS raw exhaust sampling and plume measurements for the Pre-2007 vehicle in the idle test. Plane 4 point 12, plane 6 point 12, and plane 6 point 13 present the closer DR to the raw exhaust. 


\subsubsection{20mph Test}

The setup chosen for the DDS system is summarized in Table 5-15. The total DR for this setup is 16 , and the three identified points in the plume present a DR that falls within a range of $\pm 5 \%$. The setup used in this test for the DDS was able to closely represent the PM emissions from real-world conditions for a DR of 16. A good fit is achieved for accumulation and nucleation mode. In addition, the transition between nucleation and accumulation mode is common to all the traces. No indications of maturation or coagulation effects are observed for the selected points in the plume.

Table 5-15: DDS Parameters Used During the PM Particle Size Distribution and Concentration Sampling from Raw Exhaust for the Pre-2007 Vehicle in the $20 \mathrm{mph}$ Test.

\begin{tabular}{lc}
\hline Parameter & Value \\
\hline Primary Dilution Air Temp [C] & 50 \\
\hline Primary Dilution Air Pressure [psi] & 35 \\
\hline Secondary Dilution Air Temp [C] & 40 \\
\hline Secondary Dilution Air Pressure [psi] & 35 \\
\hline Total DR & 16 \\
\hline PM spectrometer & DMS \\
\hline
\end{tabular}




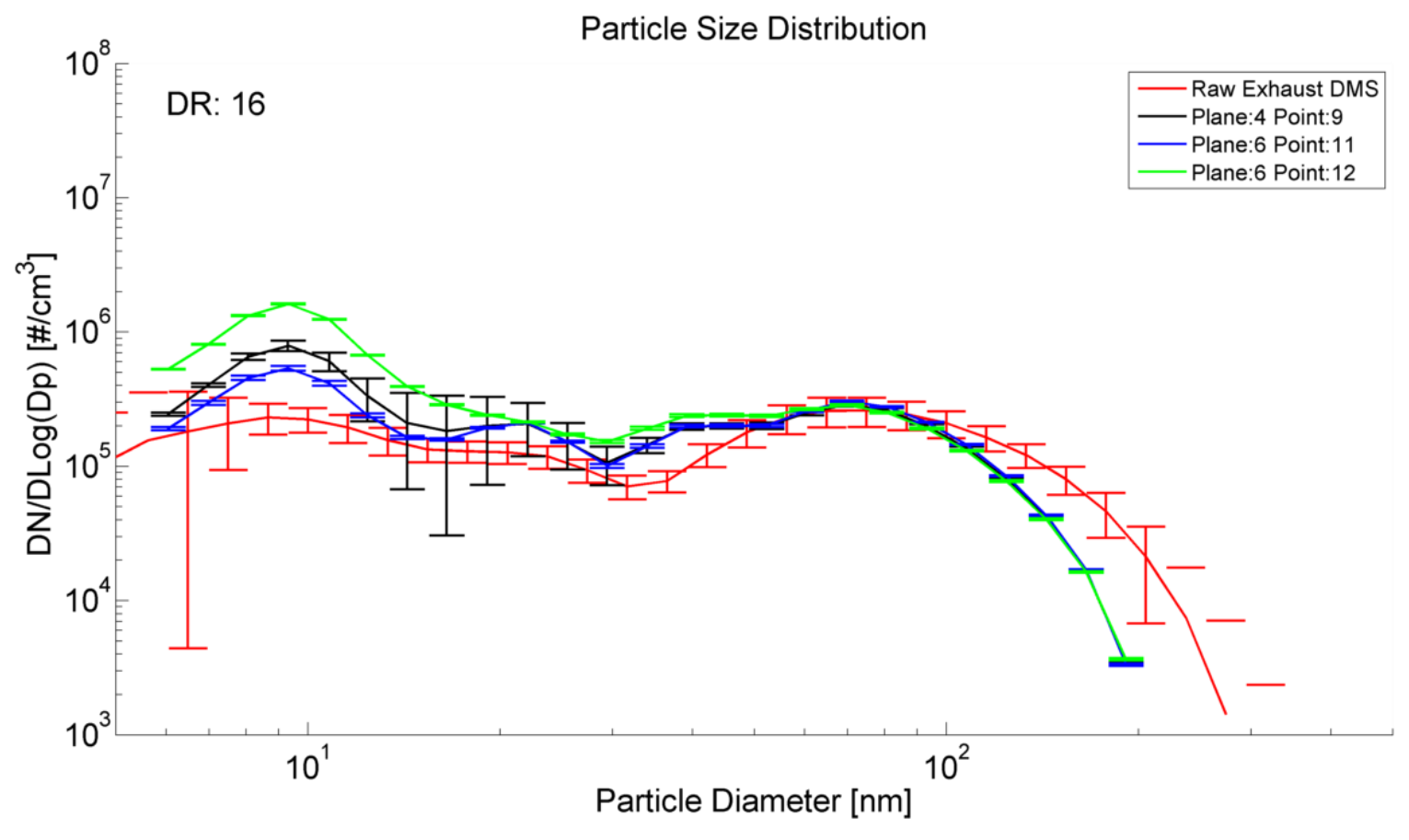

Figure 5-40: PM particle size distribution and concentration comparison between the DMS DDS raw exhaust sampling and plume measurements for the Pre-2007 vehicle in the $20 \mathrm{mph}$ Test. Plane 4 point 9, plane 6 point 11, and plane 6 point 12 present the closer DR to the raw exhaust. 


\subsubsection{35mph Test}

The setup chosen for the DDS system is summarized in Table 5-16. The total DR for this test is 17 , and the three identified points in the plume present a DR that falls within a range of $\pm 5 \%$. During this test, the SMPS was adopted instead of the DMS due to the unavailability of the DMS for this test. The results indicate good accordance between the plume points; on the contrary of the raw exhaust measurements (see Figure 5-41). The SMPS measurements indicate a single mode distribution, while the plume data indicates the presence of slight double mode particle size distributions and concentrations. On the other hand, the discrepancy between the plume point and the raw exhaust is not as strong as the idle test, and the major PM behavior is captured.

Table 5-16: DDS Parameters Used During the PM Particle Size Distribution and Concentration Sampling from Raw Exhaust for the Pre-2007 Vehicle in the $35 \mathrm{mph}$ Test.

\begin{tabular}{lc}
\hline Parameter & Value \\
\hline Primary Dilution Air Temp [C] & 50 \\
\hline Primary Dilution Air Pressure [psi] & 75 \\
\hline Secondary Dilution Air Temp [C] & 40 \\
\hline Secondary Dilution Air Pressure [psi] & 35 \\
\hline Total DR & 17 \\
\hline PM spectrometer & SMPS \\
\hline
\end{tabular}




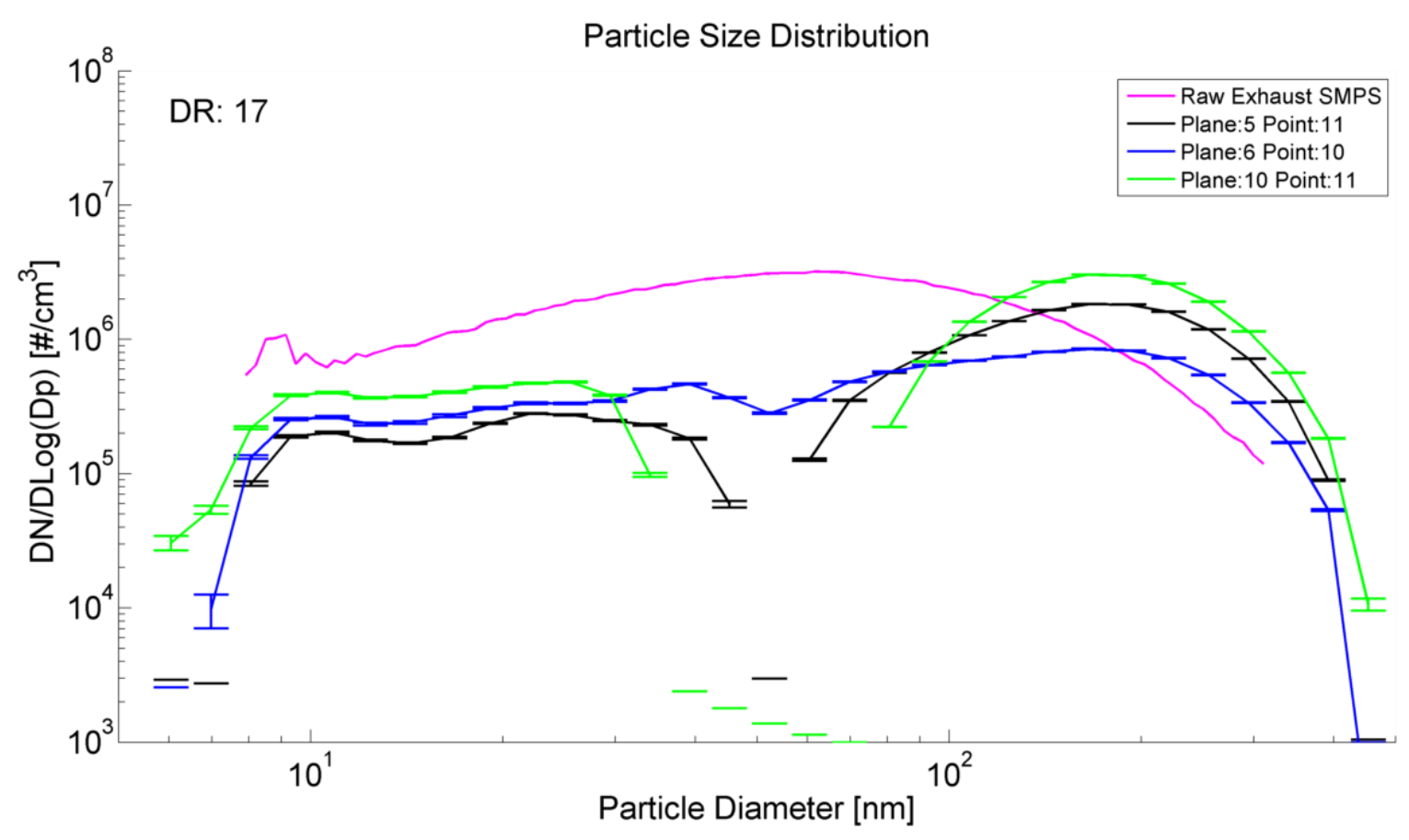

Figure 5-41: PM particle size distribution and concentration comparison between the SMPS DDS raw exhaust sampling and plume measurements for the Pre-2007 vehicle in the $35 \mathrm{mph}$ test. Plane 5 point 11 , plane 6 point 10 , and plane 10 point 11 present the closer DR to the raw exhaust. 


\subsubsection{Post-2007 Vehicle Comparison Results}

This section shows the PM emissions comparison of the Post-2007 vehicle for plume and raw exhaust measurements of PM.

\subsubsection{Idle Test}

The setup chosen for the DDS system is summarized in Table 5-17. The total DR for this setup is 9 , and the three identified points in the plume present a DR that falls within a range of $\pm 2.5 \%$. The PM emissions in this test were collected in the raw exhaust with both DMS and SMPS. While the measurements of the DDS and SMPS show a general agreement, it is not possible to claim the same for the plume data (see Figure 5-42).

Table 5-17: DDS Parameters Used During the PM Particle Size Distribution and Concentration Sampling from Raw Exhaust for the Post-2007 Vehicle in the Idle Test.

\begin{tabular}{lc}
\hline Parameter & Value \\
\hline Primary Dilution Air Temp [C] & 50 \\
\hline Primary Dilution Air Pressure [psi] & 75 \\
\hline Secondary Dilution Air Temp [C] & 40 \\
\hline Secondary Dilution Air Pressure [psi] & 75 \\
\hline Total DR & 9 \\
\hline PM spectrometer & DMS and SMPS \\
\hline
\end{tabular}


The particle size distribution and concentration of the three points in the plume shows overlap with the SMPS for the accumulation mode. On the other hand, neither the DMS nor SMPS are capable of representing the nucleation mode observed in the plume, which is short one order of magnitude. Unfortunately, the traces of the plume are missing the concentration of few particle diameter ranges, but the overall trace can be recognized.

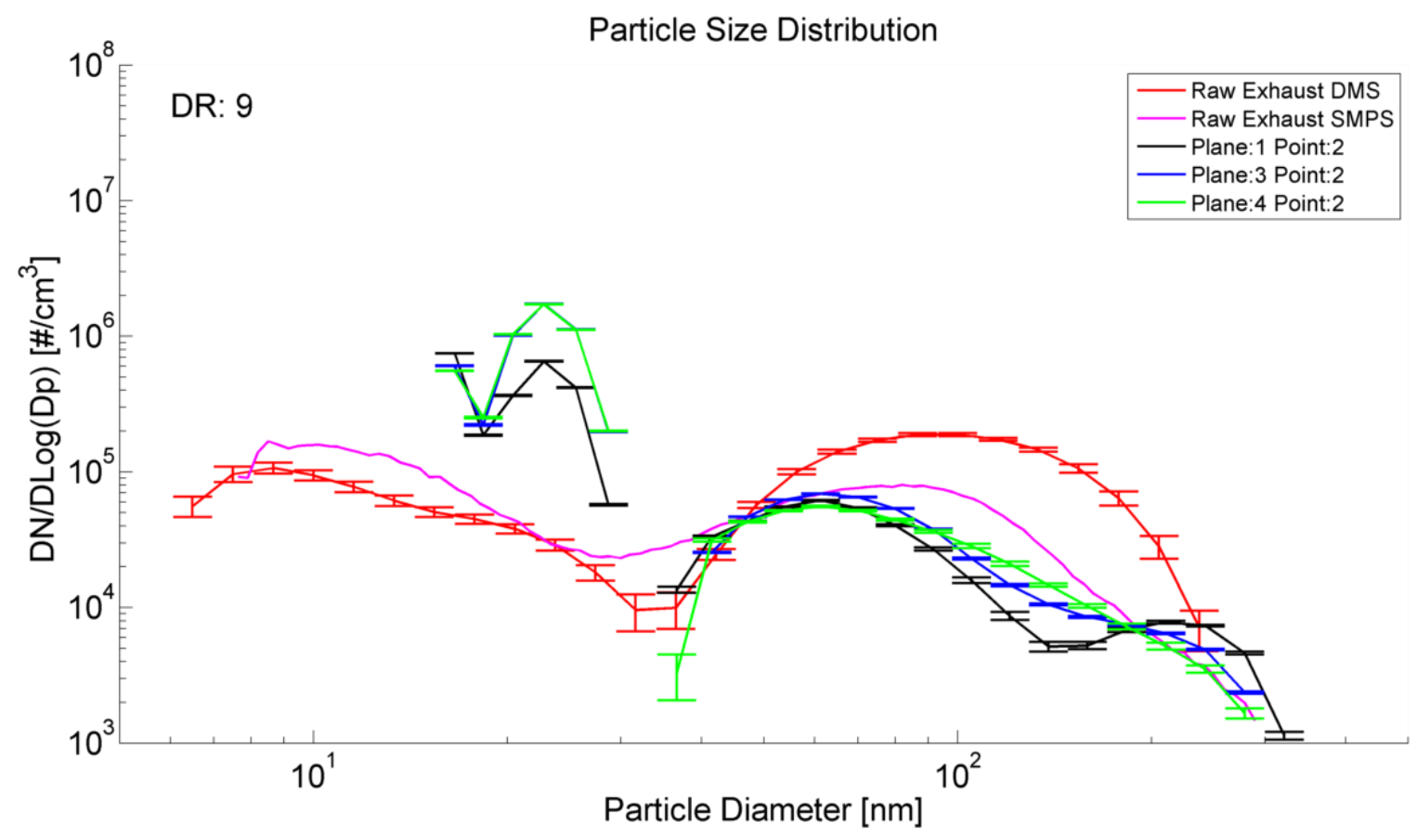

Figure 5-42: PM particle size distribution and concentration comparison between the DMS and SMPS DDS raw exhaust sampling, and plume measurements for the Post2007 Vehicle in the idle test. Plane 1 point 2, plane 3 point 2, and plane 4 point 2 present the closer DR to the raw exhaust. 


\subsubsection{20mph Test}

The setup chosen for the DDS system is summarized in Table 5-18. The total DR for this setup is 6 , and the three identified points in the plume present a DR that falls within a range of $\pm 5 \%$. The PM emissions in this test were collected in the raw exhaust with both DMS and SMPS. Both DMS and SMPS show a single mode particle size distribution and concentration, which agrees with two out of three points of the plume (see Figure 5-43). While two points of the plume overlap with the DMS trace, the SMPS overestimates the concentration for the smallest particles. The last plume point particle size distribution is completely off the other traces because it is sampled during the regeneration event, with a distinct nucleation mode and an increase in accumulation mode concentration in excess of one order of magnitude.

Table 5-18: DDS Parameters Used During the PM Particle Size Distribution and Concentration Sampling from Raw Exhaust for the Post-2007 Vehicle in the $20 \mathrm{mph}$ Test.

\begin{tabular}{lc}
\hline Parameter & Value \\
\hline Primary Dilution Air Temp [C] & 200 \\
\hline Primary Dilution Air Pressure [psi] & 35 \\
\hline Secondary Dilution Air Temp [C] & 40 \\
\hline Secondary Dilution Air Pressure [psi] & 35 \\
\hline Total DR & 6 \\
\hline PM spectrometer & DMS and SMPS \\
\hline
\end{tabular}




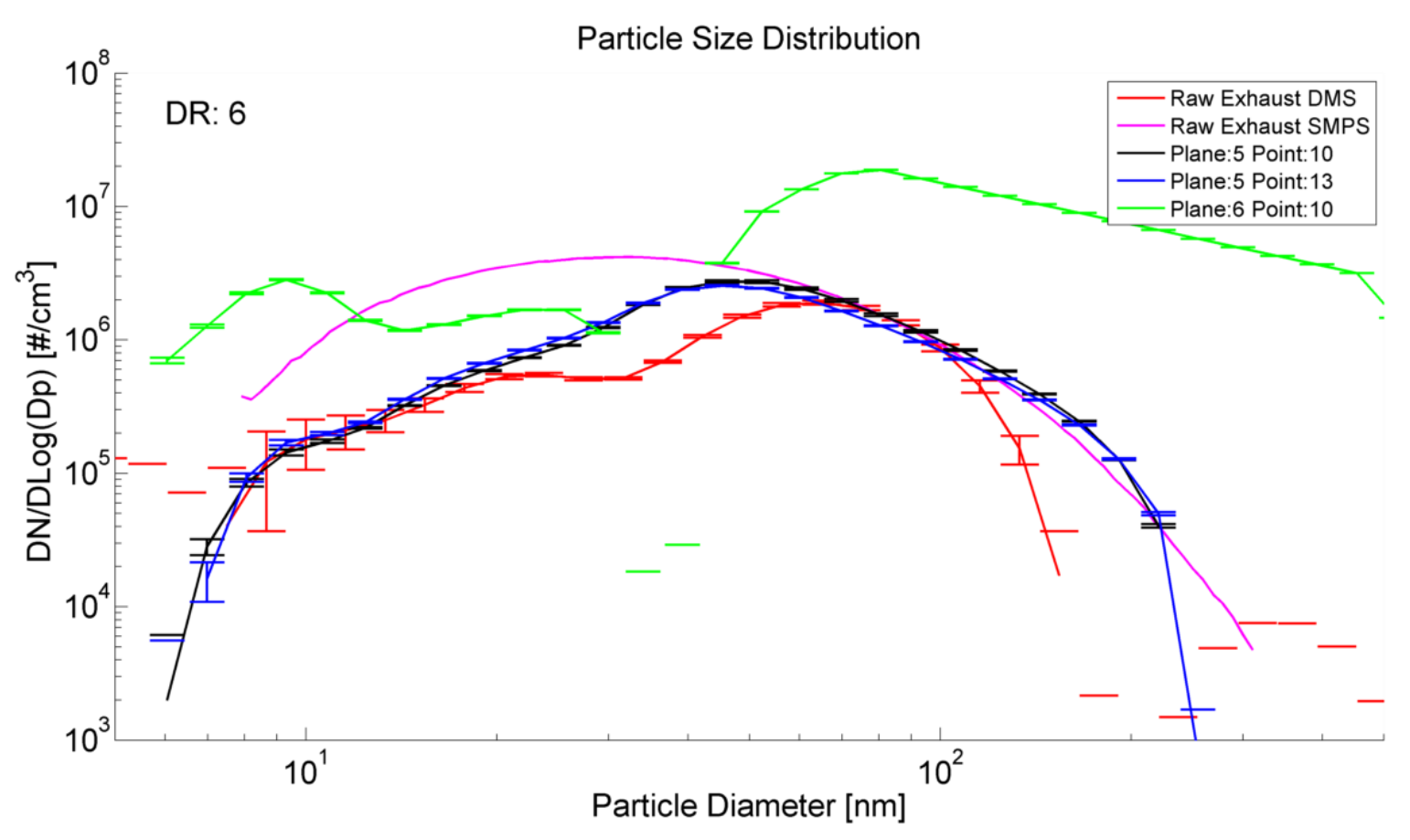

Figure 5-43: PM particle size distribution and concentration comparison between the DMS and SMPS DDS raw exhaust sampling, and plume measurements for the Post2007 vehicle in the $20 \mathrm{mph}$ test. Plane 5 point 10, plane 5 point 13, and plane 6 point 10 present the closer DR to the raw exhaust. 


\subsubsection{35mph Test}

The setup chosen for the DDS system is summarized in Table 5-19. The total DR for this setup is 8 , and the three identified points in the plume present a DR that falls within a range of $\pm 5 \%$. The DMS and SMPS traces for this test overlap for the majority of the spectrum (see Figure 5-44). While the DMS trace exhibits a slight double mode particle size distribution and concentration, the SMPS instead indicates a single mode distribution. The traces from the three sampling points of the plume agree well for the accumulation mode with DMS and SMPS, but not for the nucleation mode. The setup for raw exhaust PM measurement completely fails to represent an evident nucleation mode identified in the real-world measurement. PM particle concentration for a diameter above $300 \mathrm{~nm}$ is a residue of the background air PM.

Table 5-19: DDS Parameters Used During the PM Particle Size Distribution and Concentration for the Post-2007 Vehicle in the $35 \mathrm{mph}$ Test.

\begin{tabular}{lc}
\hline Parameter & Value \\
\hline Primary Dilution Air Temp [C] & 50 \\
\hline Primary Dilution Air Pressure [psi] & 75 \\
\hline Secondary Dilution Air Temp [C] & 40 \\
\hline Secondary Dilution Air Pressure [psi] & 35 \\
\hline Total DR & 8 \\
\hline PM spectrometer & DMS and SMPS \\
\hline
\end{tabular}




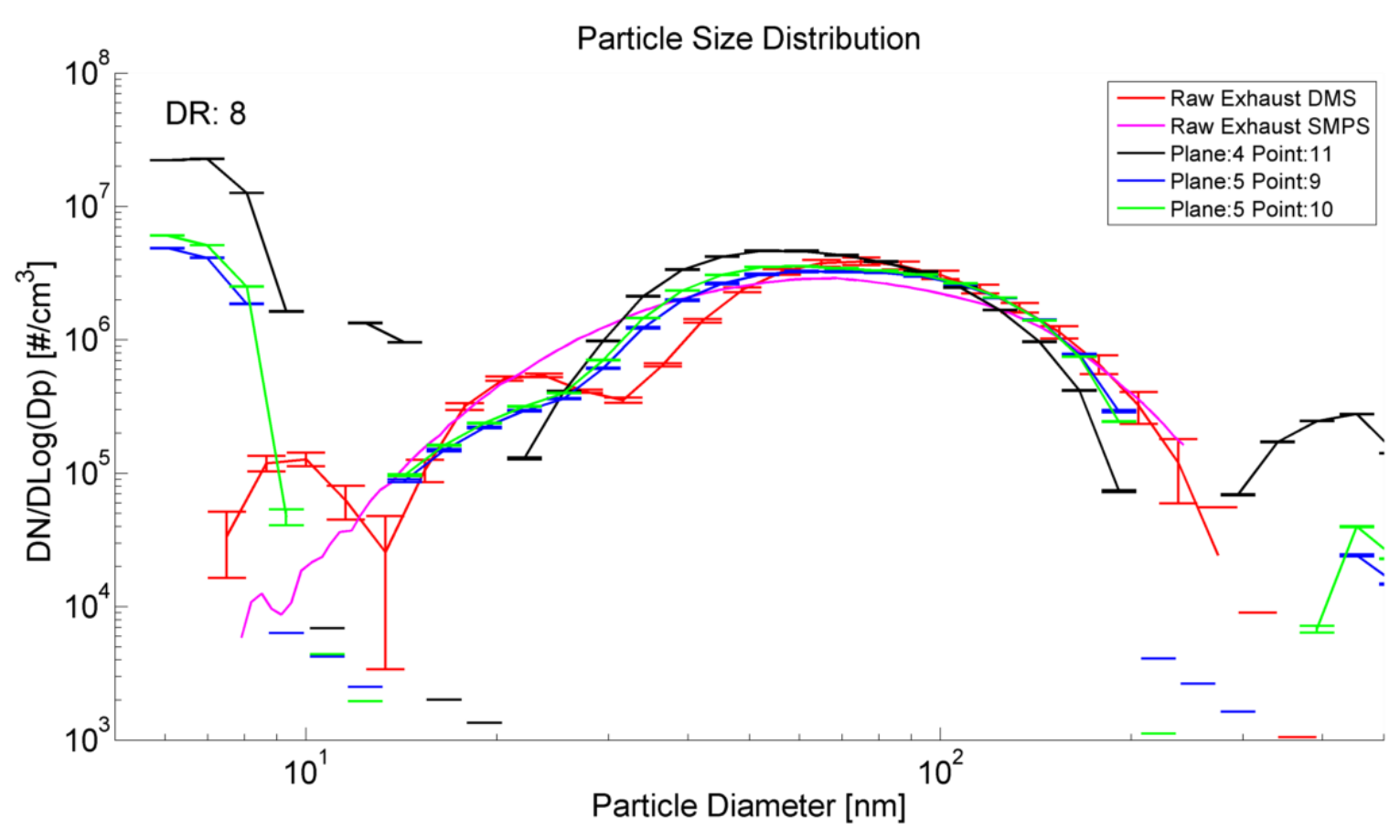

Figure 5-44: PM particle size distribution and concentration comparison between the DMS and SMPS DDS raw exhaust sampling, and plume measurements for the Post2007 vehicle in the $35 \mathrm{mph}$ test. Plane 4 point 11 , plane 5 point 9 , and plane 5 point 10 present the closer DR to the raw exhaust. 


\subsubsection{Post-2010 Vehicle Comparison Results}

This section shows the PM emissions comparison of the Post-2010 vehicle for plume and raw exhaust measurements of PM. From previous paragraphs, the PM emissions for this vehicle results were extremely low for the three wind speeds. The PM emissions resulted lower than the background air content, causing difficulties in PM sampling.

\subsubsection{Idle Test}

The setup chosen for the DDS system is summarized in Table 5-20. The total DR for this setup is 5, and the three identified points in the plume present a DR that falls within a range of $\pm 5 \%$. The comparison of PM emissions between the SMPS and plume sampling point cannot be executed, due to the EEPS operating at the lower detectable threshold and the low load generated by the engine (see Figure 5-45).

Table 5-20: DDS Parameters Used During the PM Particle Size Distribution and Concentration for the Post-2010 Vehicle in the $35 \mathrm{mph}$ Test.

\begin{tabular}{lc}
\hline Parameter & Value \\
\hline Primary Dilution Air Temp [C] & 200 \\
\hline Primary Dilution Air Pressure [psi] & 75 \\
\hline Secondary Dilution Air Temp [C] & 40 \\
\hline Secondary Dilution Air Pressure [psi] & 35 \\
\hline Total DR & 5 \\
\hline PM spectrometer & SMPS \\
\hline
\end{tabular}




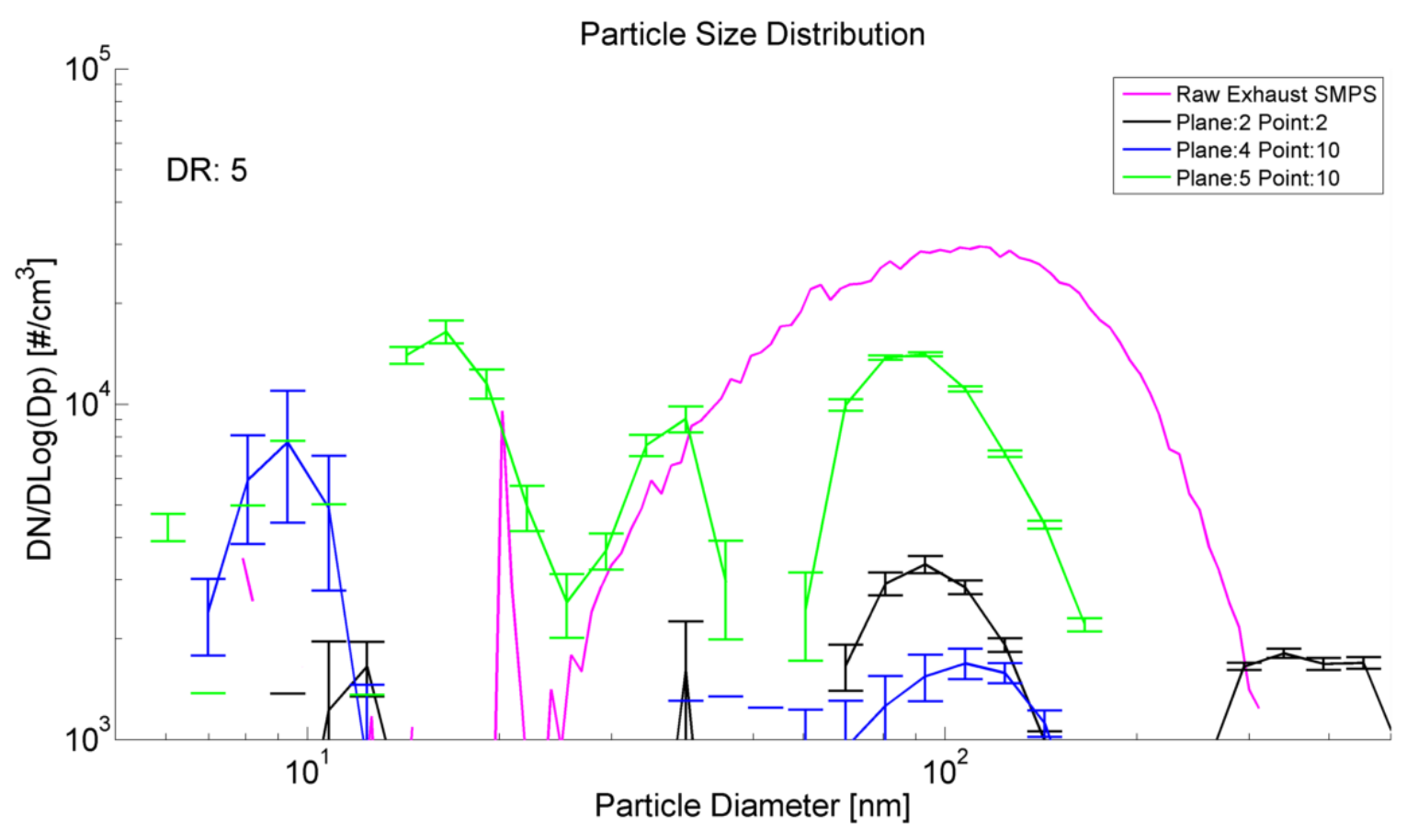

Figure 5-45: PM particle size distribution and concentration comparison between the SMPS DDS raw exhaust sampling and plume measurements for the Post2010 vehicle in the idle test. Plane 2 point 2 , plane 4 point 10 , and plane 5 point 10 present the closer DR to the raw exhaust. 


\subsubsection{20mph Test}

The setup chosen for the DDS system is summarized in Table 5-21. The total DR for this setup is 8 and the three identified points in the plume present a DR that falls within a range of $\pm 5 \%$. The engine load in this test is substantially higher with PM emissions in the plume higher than the minimum EEPS threshold. The SMPS trace is capable of following the general trend of the particle size distribution and concentration of the three points in the plume, although one order of magnitude lower for the accumulation mode. The nucleation mode difference is slightly below two orders of magnitude. Once again, the PM emissions for the three points in the plume above $300 \mathrm{~nm}$ particle diameter are related to background PM. The hump at 100nm particle diameter for the plume points are related to a contaminated electrometer within the EEPS.

Table 5-21: DDS Parameters Used During the PM Particle Size Distribution and Concentration Measurement for the 20mph Test and Post-2010 Vehicle.

\begin{tabular}{lc}
\hline Parameter & Value \\
\hline Primary Dilution Air Temp [C] & 50 \\
\hline Primary Dilution Air Pressure [psi] & 35 \\
\hline Secondary Dilution Air Temp [C] & 40 \\
\hline Secondary Dilution Air Pressure [psi] & 75 \\
\hline Total DR & 18 \\
\hline PM spectrometer & SMPS \\
\hline
\end{tabular}




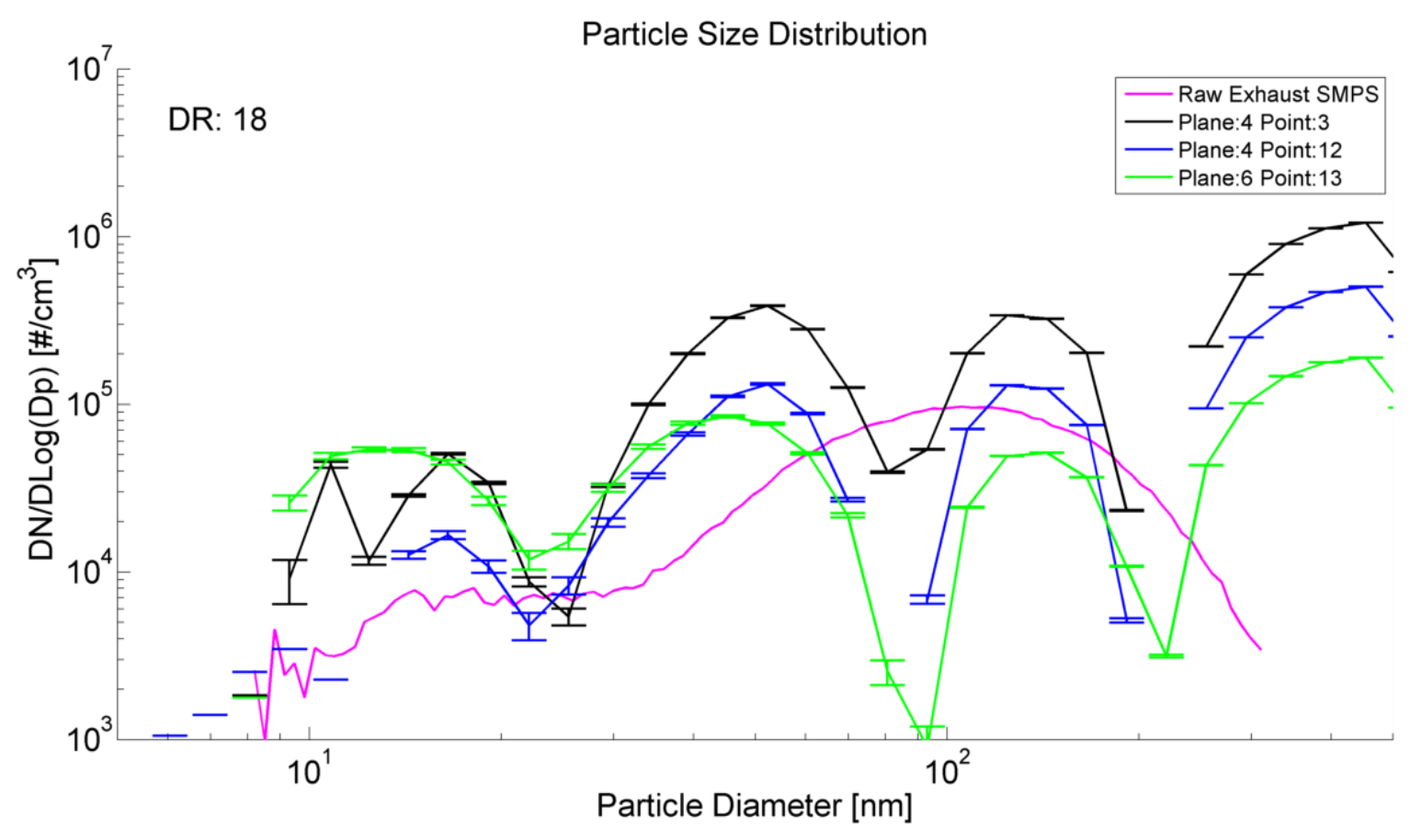

Figure 5-46: PM particle size distribution and concentration comparison between the SMPS DDS raw exhaust sampling and plume measurements for the Post2010 vehicle in the $20 \mathrm{mph}$ test. Plane 4 point 3 , plane 4 point 12 , and plane 6 point 13 present the closer DR to the raw exhaust. 


\subsubsection{35mph Test}

The setup chosen for the DDS system is summarized in Table 5-22. The total DR for this setup is 9 , and the three identified points in the plume present a DR that falls within a range of $\pm 2.5 \%$. The indications from the SMPS do not match with the particle size distributions and concentration from the plume with similar DR. On the other hand, the traces for the plume points resulted in being broken due to NaNs that the EEPS generates during the testing of this vehicle (see Figure 5-47). Those NaNs are related to measured concentrations below the lower threshold of the instrument.

Table 5-22: DDS Parameters Used During the PM Particle Size Distribution and Concentration Measurement for the 35mph Test and Post-2010 Vehicle.

\begin{tabular}{lc}
\hline Parameter & Value \\
\hline Primary Dilution Air Temp [C] & 50 \\
\hline Primary Dilution Air Pressure [psi] & 75 \\
\hline Secondary Dilution Air Temp [C] & 40 \\
\hline Secondary Dilution Air Pressure [psi] & 35 \\
\hline Total DR & 9 \\
\hline PM spectrometer & SMPS \\
\hline
\end{tabular}




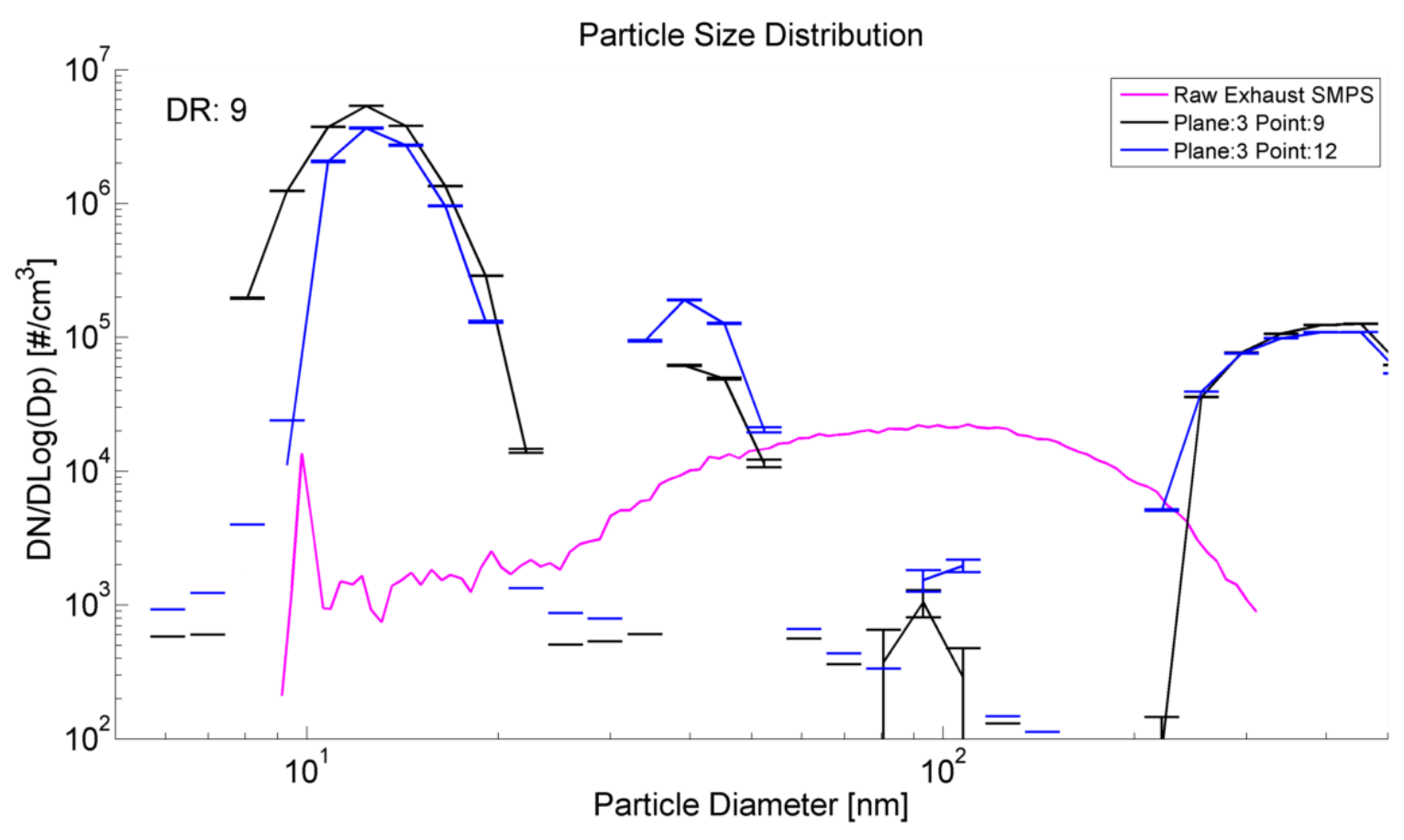

Figure 5-47: PM particle size distribution and concentration comparison between the SMPS DDS raw exhaust sampling and plume measurements for the Post2010 vehicle in the $35 \mathrm{mph}$ test. Plane 3 point 9 , plane 3 point 12 present the closer DR to the raw exhaust. 


\section{Chapter 6: Detailed Plume Modeling via 3D MARS}

This chapter further investigates the assumptions and speculations proposed in the previous chapter, which focuses on the area of the plume with higher TI generation as it pertains to the majority of nucleation mode particles in the plume. The homogeneity of the particle size distribution and concentration tends to diminish and disappear during the initial stages of plume development, due to the continuous mixing of the truck's wake. The MARS code identifies two main paths across the plume; the path with higher TI and the core of the plume. Particle size distribution and concentration along these two paths will be compared to provide evidence for the evolution and maturation of PM.

\subsection{MARS CODE DEVELOPMENT}

MARS, or Multivariate Adaptive Regression Splines, was developed by Jerome H. Friedman (Friedman, 1990) (Friedman, 1993). It is a nonparametric method that enables the researcher to incorporate flexible regression modeling of multidimensional data; i.e. the predictor does not take a predetermined form, but it is constructed according to information derived from resultant data. Product spline basis functions are carried over, but they are carried over differently from other regression methodologies; the number of basis functions as well as parameters assigned to each one are related to the data (Friedman, 1990) (Friedman, 1993) (Velardi, 2013). The goal of the algorithm is to produce a minimal set of basis functions, or $\mathrm{B}_{\mathrm{M}}$, to approximate each output function. This is accomplished through a two-phase iterative approach. The first phase, also called forward part, generates a superset of basis functions. The data is then over-fitted and the number of basis functions 
is found to be larger than optimal. The second phase, backward procedure, selectively deletes basis functions with the goal of producing an acceptable fitting with the number of bases (Velardi, 2013). Mario Velardi, during his dissertation on the analysis of fuel parameters on gaseous and PM emissions of medium-duty diesel engines, developed the code that will also be employed for this research. A variety of statistical tools and software were compared with results from his research in order to provide verification. Both Mario Velardi, and the author of this research, fine-tuned the MARS model to better model and record characteristics of the plume to be studied. The MARS code generates a 3D model function of $x_{-}^{-}, y_{-}$, and $\mathrm{z}$-coordinates for each of the 45 channels measured from the plume, as shown below:

1. $\mathrm{X}[\mathrm{m}]$

2. $\mathrm{Y}[\mathrm{m}]$

3. $\mathrm{Z}[\mathrm{m}]$

4. $\mathrm{CO}_{2}[\%]$

5. $\mathrm{NO}_{\mathrm{x}}[\mathrm{ppm}]$

6. Temperature $[\mathrm{k}]$

7. Wind Speed $[\mathrm{m} / \mathrm{s}]$

8. Turbulence Intensity [\%]

9. $\mathrm{NO}_{\mathrm{x}}$ based Local Dilution Ratio

10. DustTrack $\left[\mathrm{mg} / \mathrm{m}^{3}\right]$

11. Aethalometer $\left[\mathrm{ng} / \mathrm{m}^{3}\right]$

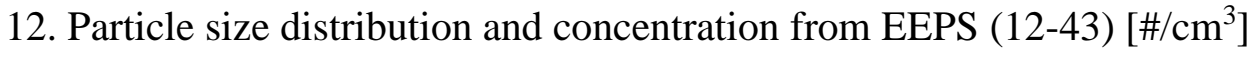

44. Total particle count $\left[\# / \mathrm{cm}^{3}\right]$

45. Total particle count nucleation mode $\left[\# / \mathrm{c} \mathrm{m}^{3}\right]$

46. Total particle count accumulation mode [\#/c $\left.\mathrm{m}^{3}\right]$

47. CMD for the nucleation mode [nm] 
48. CMD for the accumulation mode [nm]

The model is generated using 131 sampling points obtained during the rigorous testing campaign. Boundary condition points were also added to improve model accuracy outside of the plume. These boundary condition points accurately depict and reflect background air parameters.

Due to the extensive description of gaseous and solid PM emissions found in the plume in previous chapters of this dissertation, this chapter will solely focus on particle size distribution, concentration, and evolution in the plume along these two, aforementioned paths. The TI path is identified by the $\mathrm{x}, \mathrm{y}$, and $\mathrm{z}$ positioning of the highest TI values, while the core path is identified by the highest values of $\mathrm{NO}_{\mathrm{x}}$ emission. The two paths are chosen as the one with the highest and lowest chances of nucleation mode formation. The high TI path collect the position of the region with high TI, where the peak of nanoparticles and nucleation mode concentration was observed. The path with highest $\mathrm{NO}_{\mathrm{x}}$ emission, identify the core of the plume, where slower dilution and cooling processes provide less chances for the nucleation to occur.

Aside from the 48 channels, the respective standard deviations were modeled, however, due to their erratic behavior- the results are deemed unreliable and erroneous, since are not able to replicate the input data.

The greatest advantage and perk of the MARS modeling method, in comparison to the interpolated results of the previous chapter, is the continuous tracing of plume information as it is definite with analytical functions. This characteristic greatly enables and bolsters the success of the researcher to identify PM evolution as it occurs. 


\subsection{Comparison of PM Particle Size Distribution and Concentration Upon Paths with Predominant Nucleation and Accumulation Mode}

\subsubsection{Pre-2007 Vehicle MARS Results}

\subsubsection{Idle Test}

The first quality check for the generated model is to interrogate the model itself on the plume points from whence it was generated and evaluate the emissions in those points. Those results from the model are compared to original set of data gathered from the plume during the tests. A simple and efficient, yet effective way to compare models data and test data is to use the residual sums of squares method $\left(\mathrm{R}^{2}\right)$. Figure 6- indicates MARS is able to achieve a $\mathrm{R}^{2}$ above 0.9 for the majority of the channels. Two channels fall below 0.9 , which are the plume temperature and the particle concentrations of the EEPS for the $5 \mathrm{~nm}$ particle diameter.

To simplify the definition of points along the two paths, the same z-coordinates of the sampling planes are going to be used, with the same denomination. Starting with the analysis of Figure 6-2 it is possible to observe how different the traces are for the two paths at plane 1, especially regarding the nucleation mode. The dash-dot traces of Figure 6-2 are similar, indicating turbulent mixing as it eventually homogenizes the plume. It is noticed that the accumulation mode for plane 1 overlaps for both paths, and a good correlation is present for the same at plane 7 . 
On the other hand, there is not a similarity between plane 1 and plane 7 accumulation modes, and the nucleation mode concentration is higher in plane 7 than plane 1 . These indications are explainable by a shift effect for PM modeling far from the exhaust stack. Thus, these discrepancies could be solved considering a shift downward of the plane 7 plots of one order of magnitude to match the accumulation mode of plane 1. As a result, the nucleation mode of plane 7 would seat between the two traces of the nucleation mode for plane 1.

The trace of plane 1 results less smooth than the ones of plane 7 due to the smaller plume dimensions and greater gradients that the model has to depict.

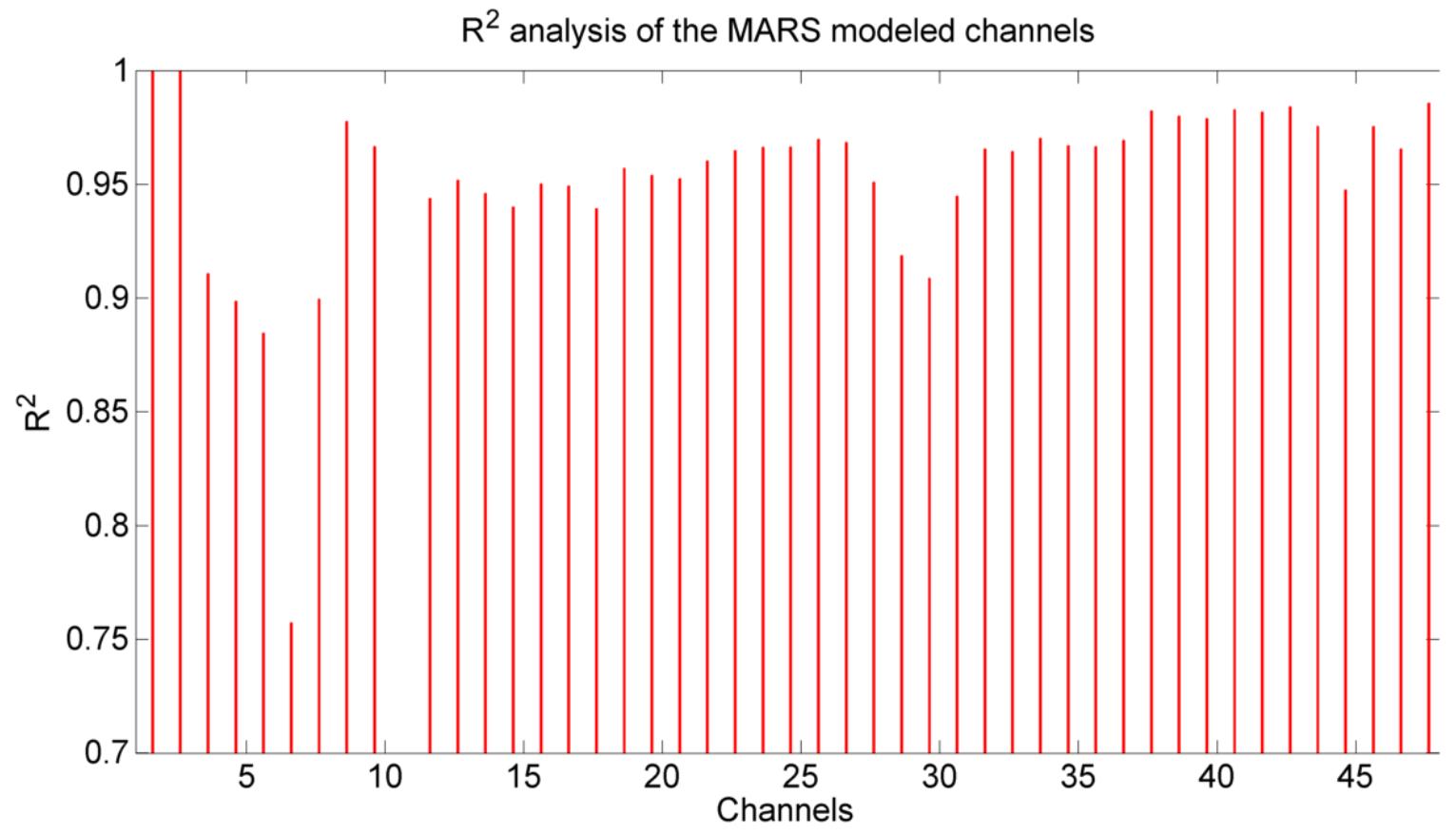

Figure 6-1: Residual sum of squares analysis for the 48 channels for the Pre-2007 vehicle during the idle test. 
To better depict the evolution of the PM on the two paths, Figure 6-3 compares the particle size distribution and concentration along five planes for the two paths. Once again, it is possible to observe an increase in nucleation mode for the plume core path and a reduction for the high TI path. This it even more visible if we shift downward the traces for planes far from the exhaust.

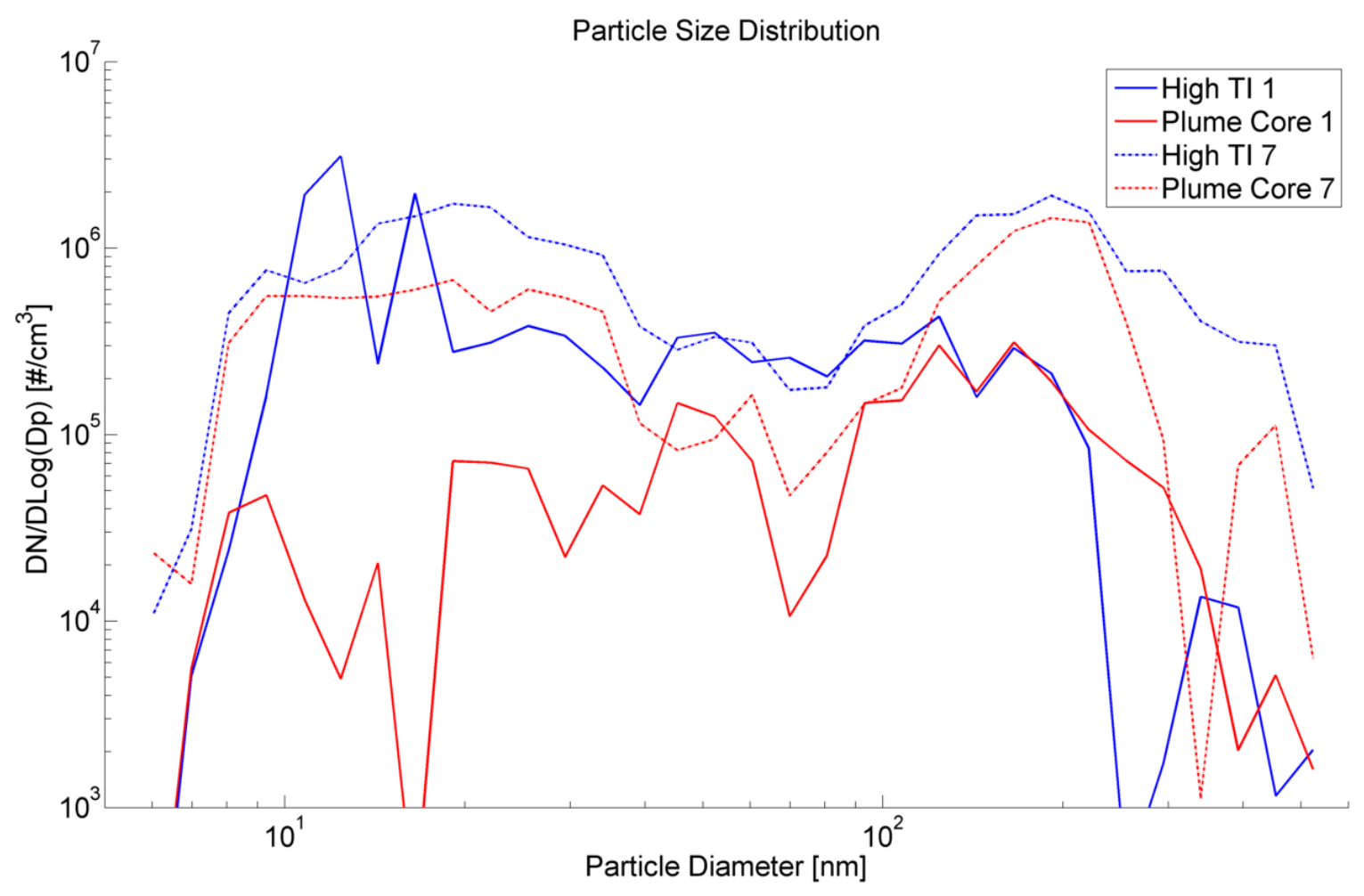

Figure 6-2: Particle size distribution and concentration for plane 1 and plane 7 of the TI path and core plume path (respectively $0.115 \mathrm{~m}$ and $2.815 \mathrm{~m}$ from the exhaust stack). This plot represent the results of the MARS model for the Pre-2007 vehicle during the idle test. 

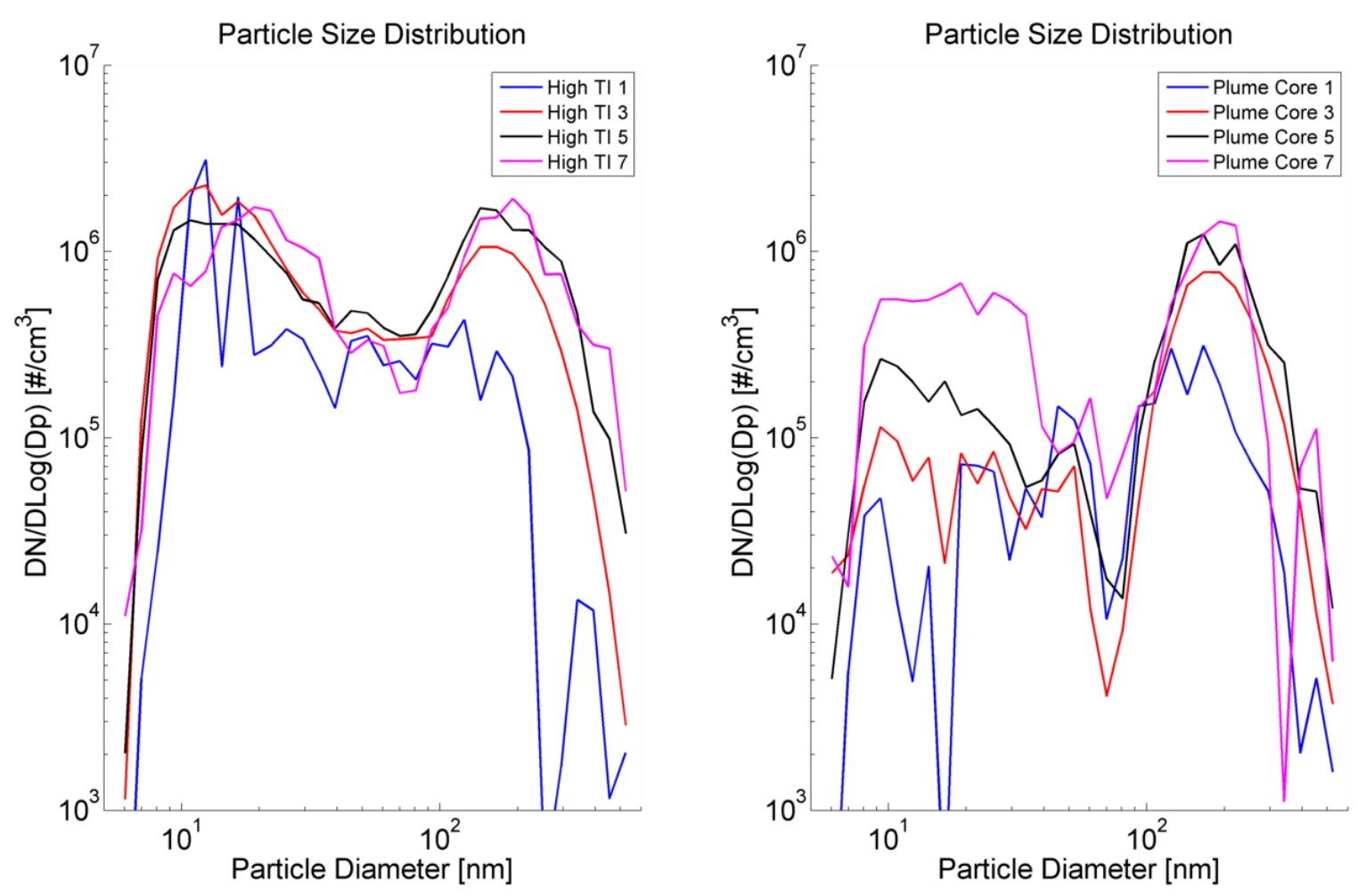

Figure 6-3: Particle size distribution and concentration for plane 1 to plane 7 of the TI path and core plume path (respectively from $0.115 \mathrm{~m}$ to $2.815 \mathrm{~m}$ after the exhaust stack). This plot represent the results of the MARS model for the Pre-2007 vehicle during the idle test. 


\subsubsection{2. $20 \mathrm{mph}$ Test}

The model residual sum of squares for this combination of vehicle and wind speed is above 0.85 for the large part of the channels, and only a few fall below this value. This result is considered acceptable due to the complexity of modeling an evolving plume (see Figure 6-4).

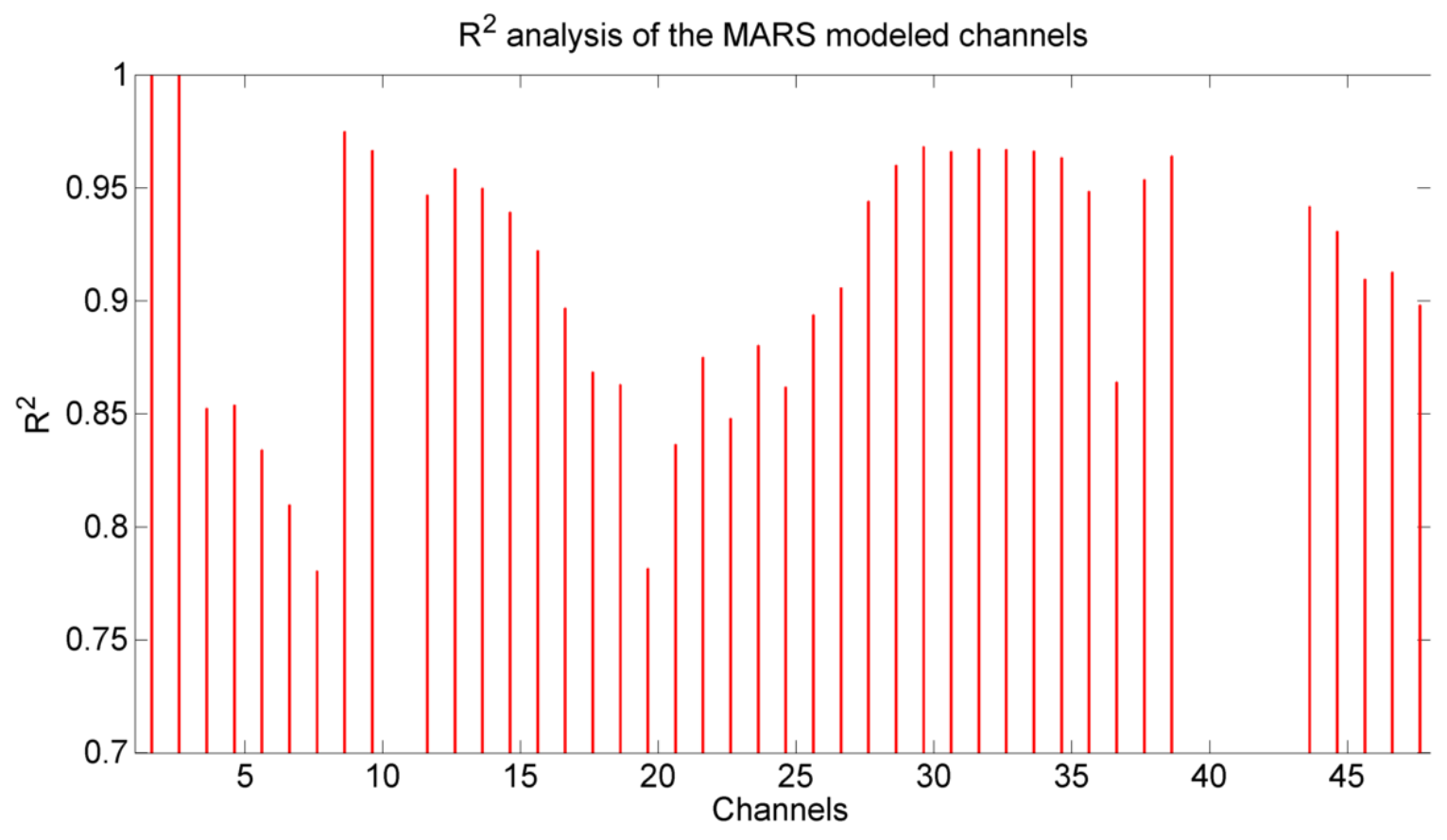

Figure 6-4: Residual sum of squares analysis for the 48 channels for the Pre-2007 vehicle during the $20 \mathrm{mph}$ test.

The particle size distribution and concentration for plane 1 represent faithfully what was observed during the interpolation results of the previous chapter. In Figure 6-5, the path of high TI on plane 1 presents higher concentration of nucleation modes if compared to the plume core path. The plume core presents a single mode distribution, related to a 
dilution process that did not allow the nucleation mode to appear. Moving further down the plume, plane 7 identifies overlapping PM particle size distribution and concentration for the two paths, with a distinct nucleation mode, one order of magnitude greater than the accumulation mode.

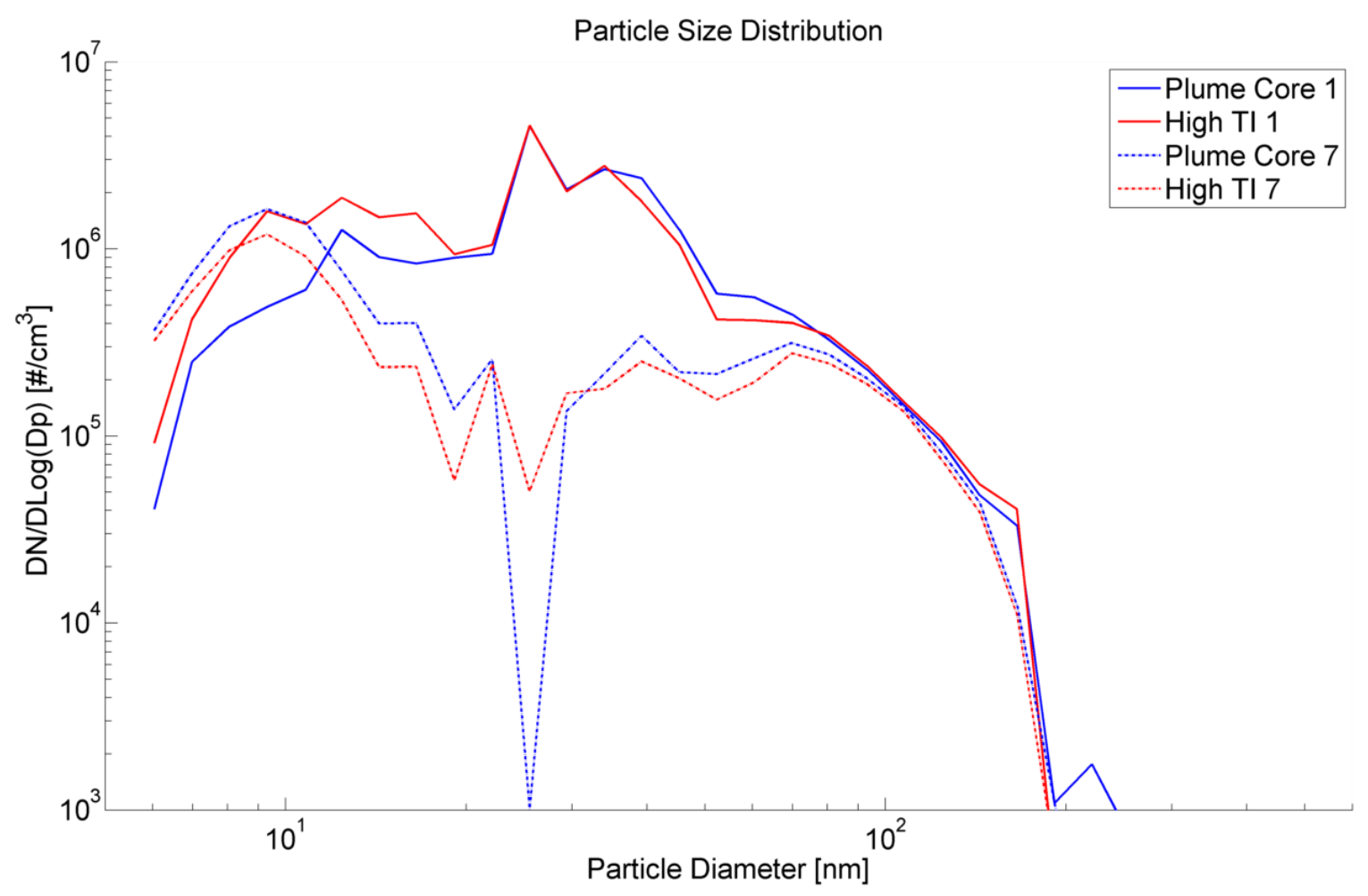

Figure 6-5: Particle size distribution and concentration for plane 1 and plane 7 of the TI path and core plume path (respectively $0.115 \mathrm{~m}$ and $2.815 \mathrm{~m}$ from the exhaust stack). This plot represents the results of the MARS model for the Pre-2007 vehicle during the $20 \mathrm{mph}$ test. 

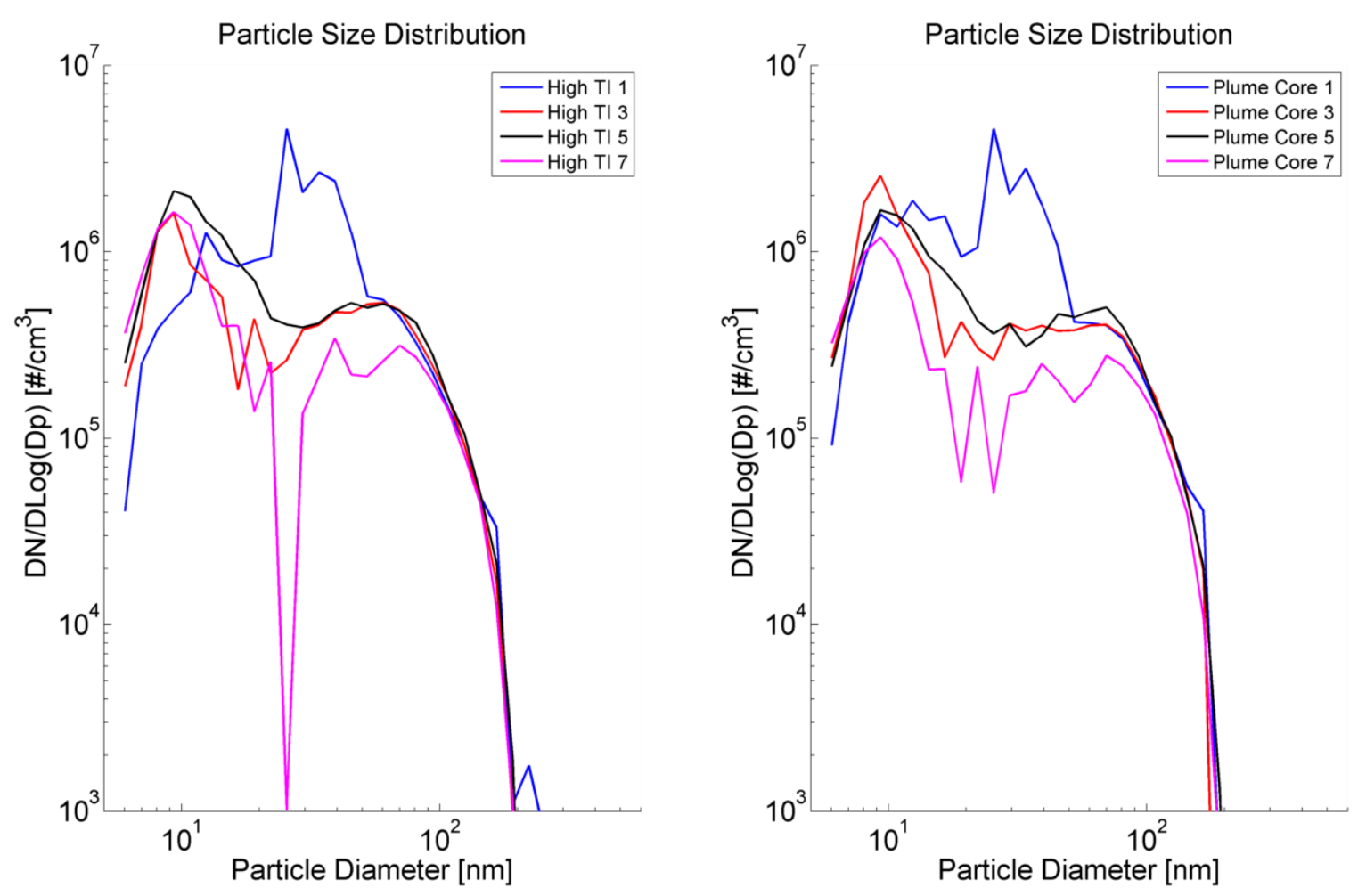

Figure 6-6: Particle size distribution and concentration for plane 1 to plane 7 of the TI path and core plume path (respectively from $0.115 \mathrm{~m}$ to $2.815 \mathrm{~m}$ after the exhaust stack). This plot represents the results of the MARS model for the Pre-2007 vehicle during the $20 \mathrm{mph}$ test.

The PM particle concentration in the diameter range from $20 \mathrm{~nm}$ to $50 \mathrm{~nm}$ experiences an important evolution from plane 1 to plane 7 , it is speculated that the transition region between accumulation and nucleation modes suffers coagulation and absorption. The MARS model for the PM concentration at $30 \mathrm{~nm}$ generates a relatively unexpected behavior in all four traces, related to a non-satisfactory model adherence to sampled data $\left(R^{2}<0.7\right)$. 
Figure 6-6 provides information on the evolution of the PM particle size distribution and concentration for the two paths. In both cases, it is evident how the traces of both paths initially different while moving downwind the plume tend to have an overlapping trace, as it is possible to see in Figure 6-5 in a simpler way for the traces of plane 7.

\subsubsection{35mph Test}

The last test of this vehicle presents a model with a residual sum of squares for the majority above 0.85 , and few channels below the same value (see Figure 6-7). The channels of the PM particle size distribution and concentration have an $\mathrm{R}^{2}$ above 0.85 and their models are considered acceptable. 


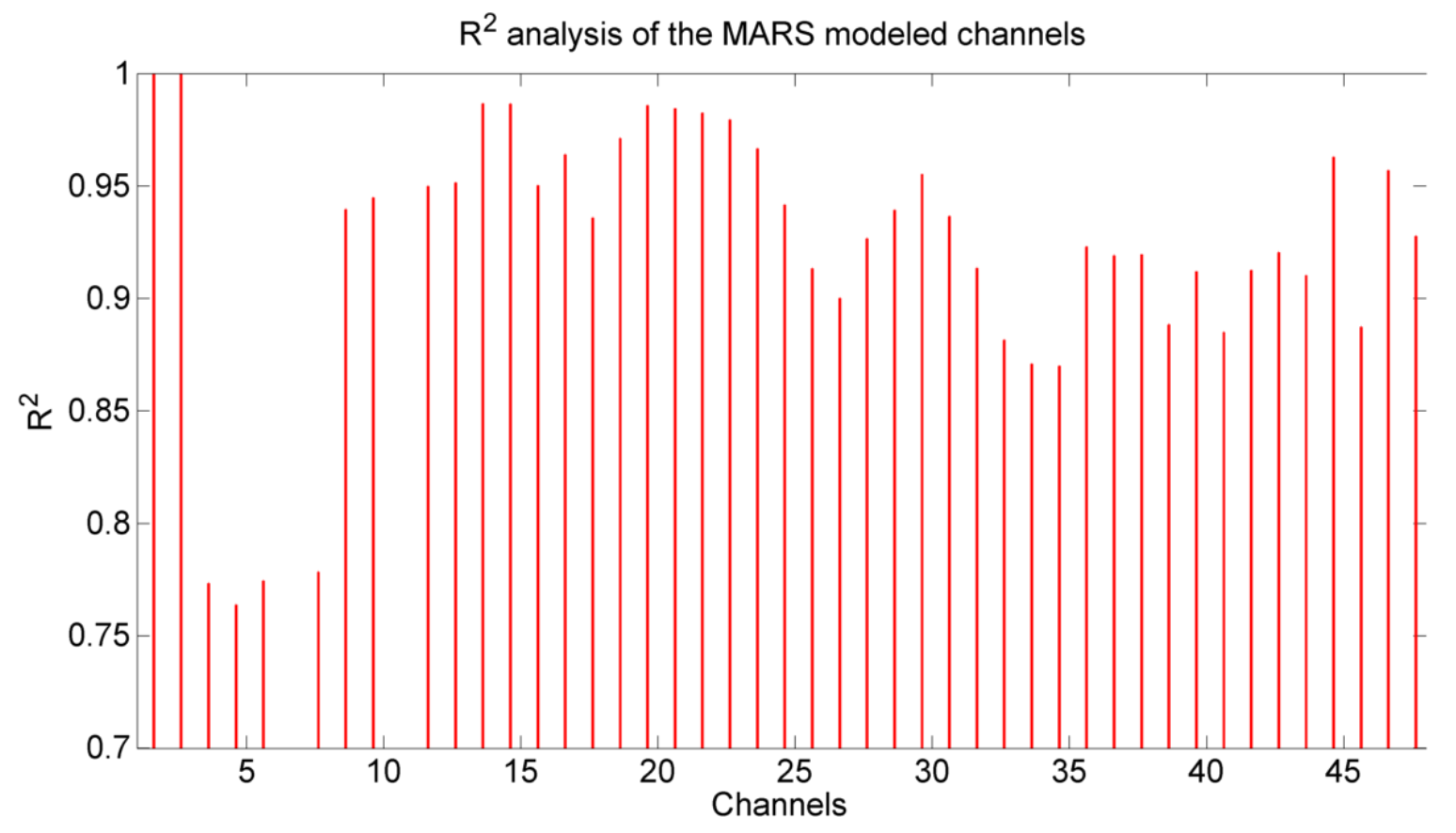

Figure 6-7: Residual sum of squares analysis for the 48 channels for the Pre-2007 vehicle during the $35 \mathrm{mph}$ test. 


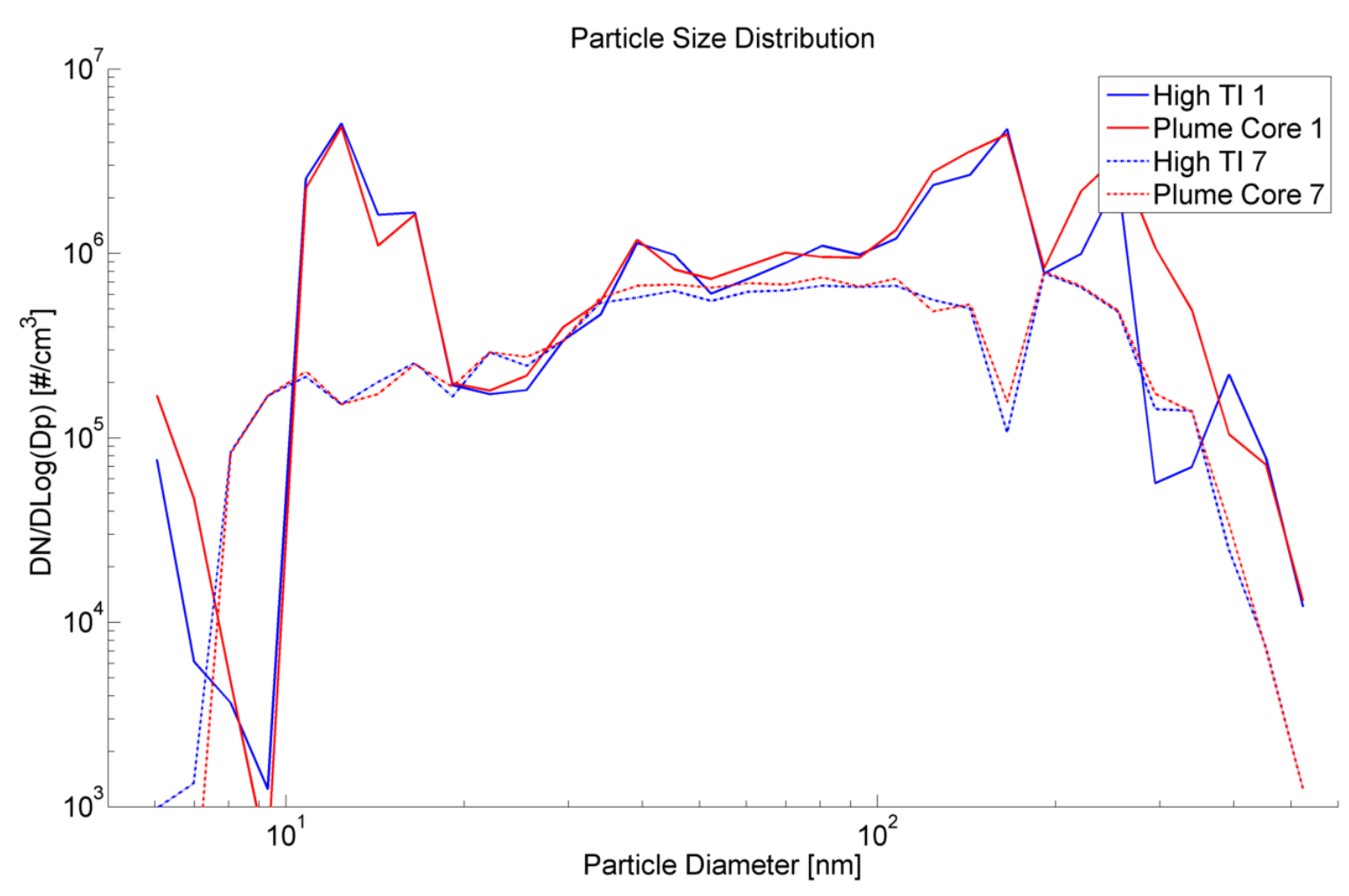

Figure 6-8: Particle size distribution and concentration for plane 1 and plane 7 of the TI path and core plume path (respectively $0.115 \mathrm{~m}$ and $2.815 \mathrm{~m}$ from the exhaust stack). This plot represents the results of the MARS model for the Pre-2007 vehicle during the $35 \mathrm{mph}$ test.

At plane 1, both paths exhibit the formation of nucleation mode. This is symptomatic and evidence of enough turbulent energy present in the wind to generate the nucleation mode independently of the position in the plume (see Figure 6-8). In addition, this indicates that the formation of the nucleation mode takes place at the exhaust stack. On the other hand, the high content of solid PM (soot) in the plume tends to absorb the newborn nucleation mode, and no trace of it is present at plane 7 for both paths. 
Figure 6-9 confirms the indication that the newborn nucleation mode (observed across the entire plume) is rapidly adsorbed from the soot, and an initial double mode distribution becomes a single mode distribution.
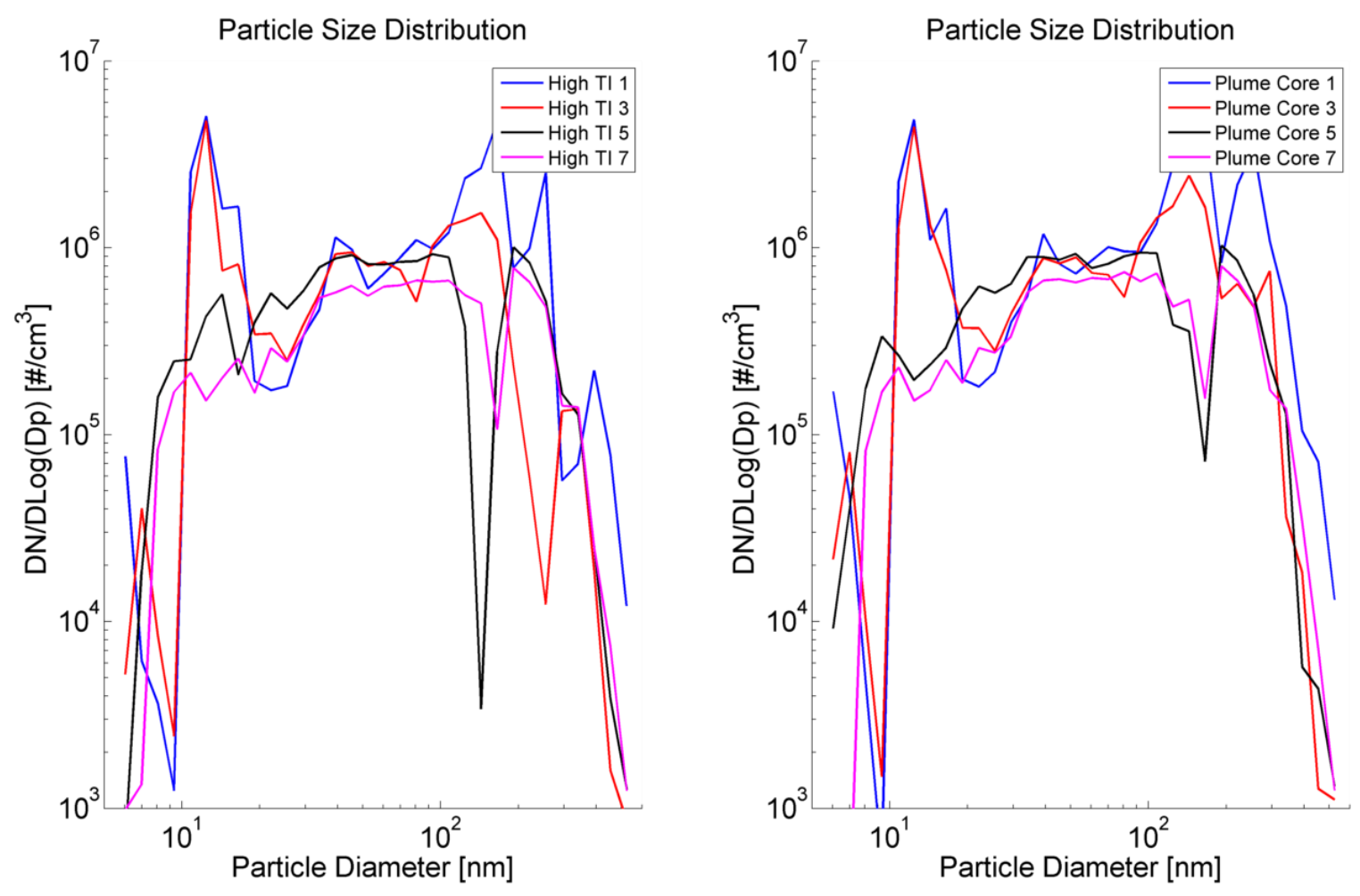

Figure 6-9: Particle size distribution and concentration for plane 1 to plane 7 of the TI path and core plume path (respectively from $0.115 \mathrm{~m}$ to $2.815 \mathrm{~m}$ after the exhaust stack). This plot represents the results of the MARS model for the Pre-2007 vehicle during the $35 \mathrm{mph}$ test. 


\subsubsection{Post-2007 Vehicle MARS Results}

\subsubsection{Idle Test}

The first test of this vehicle presents a model with a residual sum of squares for the majority above 0.85 , and few channels below the same value (see Figure 6-10).

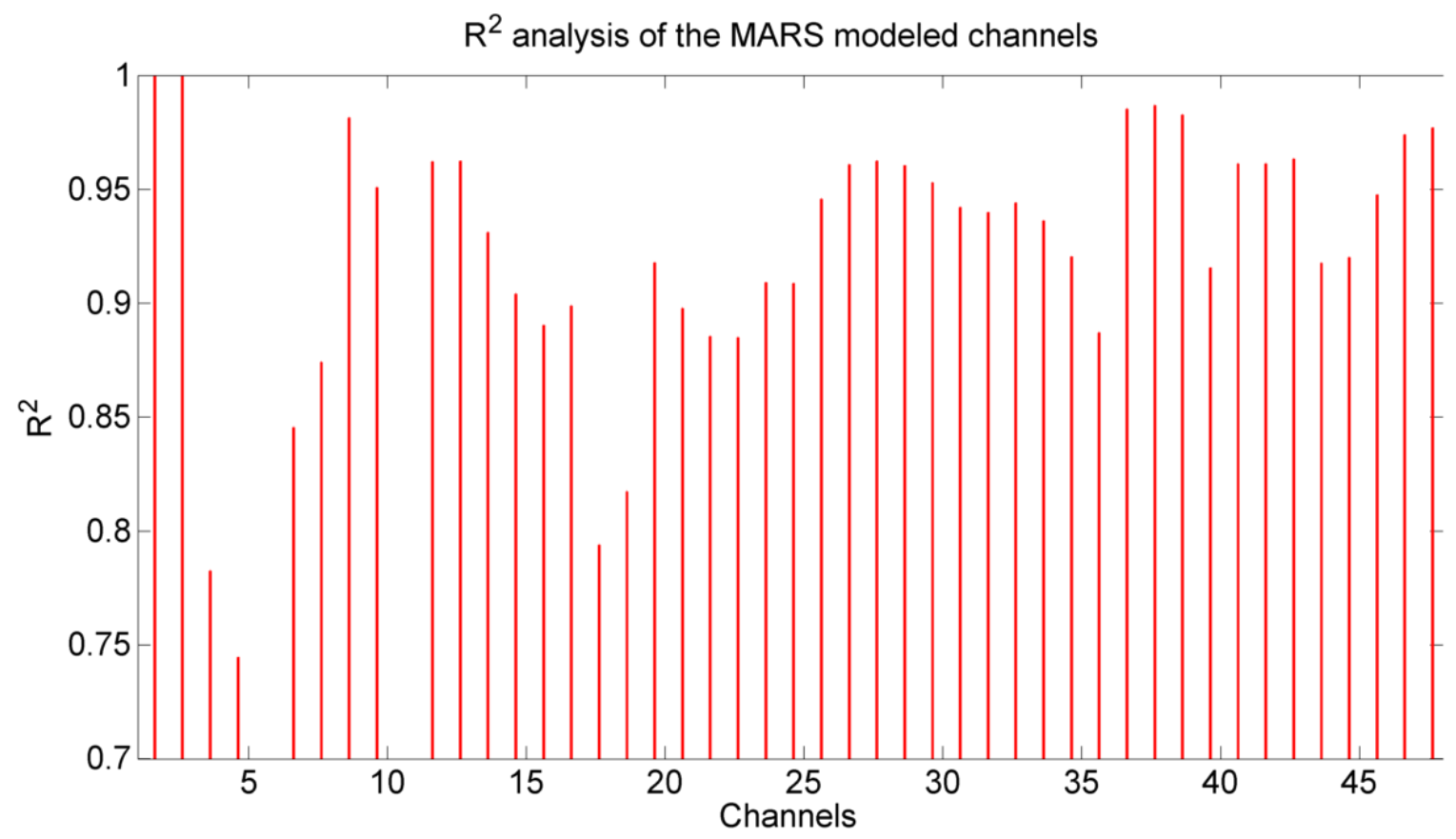

Figure 6-10: Residual sum of squares analysis for the 48 channels for the Post-2007 vehicle during the idle test.

The range of channels of the PM particle size distribution and concentration (12 to 43) results satisfactory with a $\mathrm{R}^{2}$ above 0.85 . Figure 6-11 shows that the particle size distribution for plane 1 in for both paths are very similar, with a distinctive nucleation mode. Such an indication reflects that already in early stages the reduced soot concentration favorites the nucleation mode over the entire plume section. At plane 7 of Figure 6-11, it 
is easy to depict that the PM particle size distribution and concentration do not experience any major modifications.

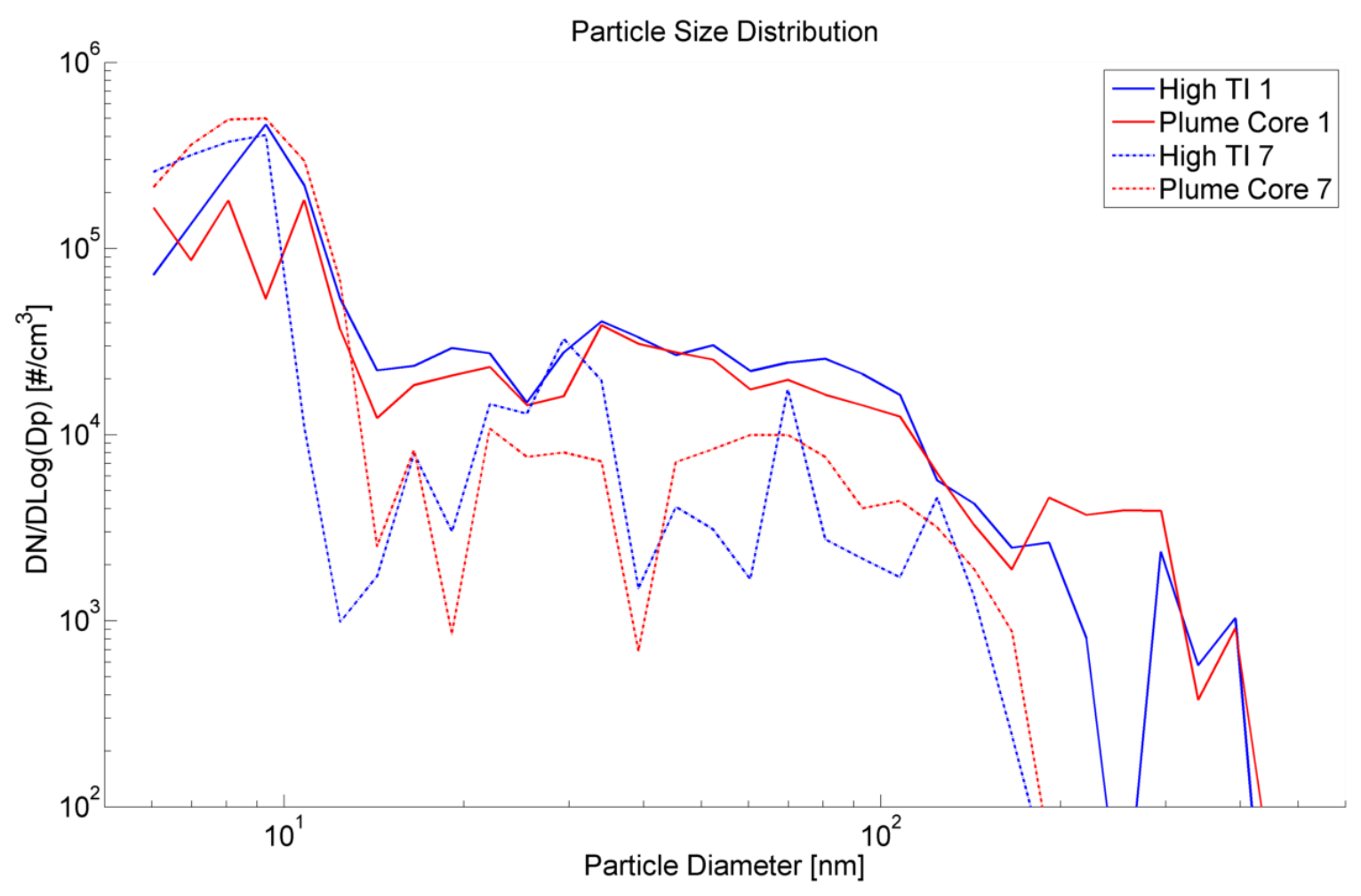

Figure 6-11: Particle size distribution and concentration for plane 1 and plane 7 of the TI path and core plume path (respectively $0.115 \mathrm{~m}$ and $2.815 \mathrm{~m}$ from the exhaust stack). This plot represents the results of the MARS model for the Post-2007 vehicle during the idle test.

Figure 6-12 shows the evolution across seven planes of the PM particle distribution and concentration, indicating that all the traces overlap for both accumulation and nucleation modes. This confirms again that the formation of the nucleation mode takes places right at the exit of the exhaust stack across the entire plume. 

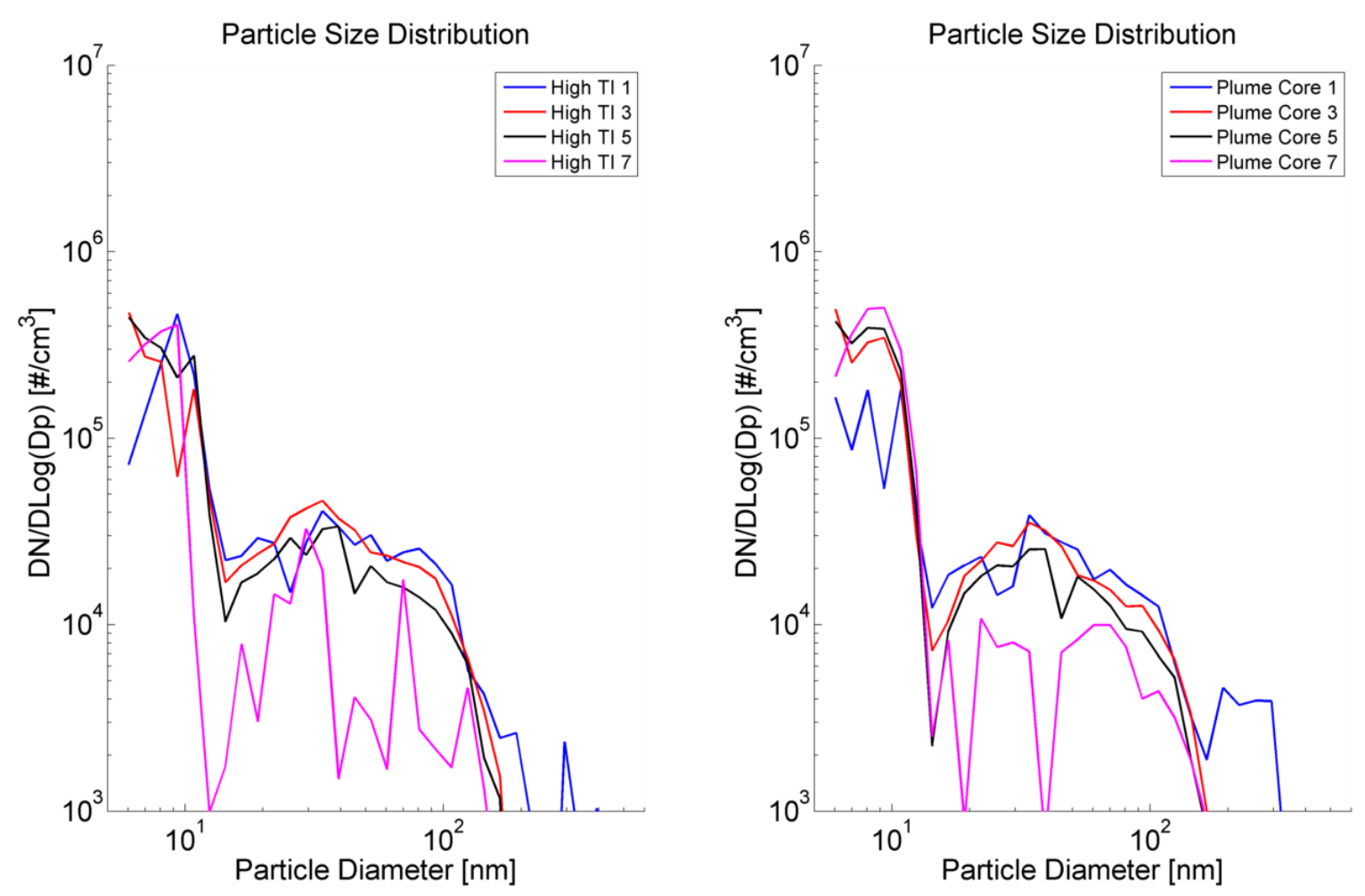

Figure 6-12: Particle size distribution and concentration for plane 1 to plane 7 of the TI path and core plume path (respectively from $0.115 \mathrm{~m}$ to $2.815 \mathrm{~m}$ after the exhaust stack). This plot represents the results of the MARS model for the Post-2007 vehicle during the idle test. 


\subsubsection{20mph Test}

The residual sum of squares for this test indicates that the channel models of the PM particle size distribution and concentration are above 0.85 (see Figure 6-13).

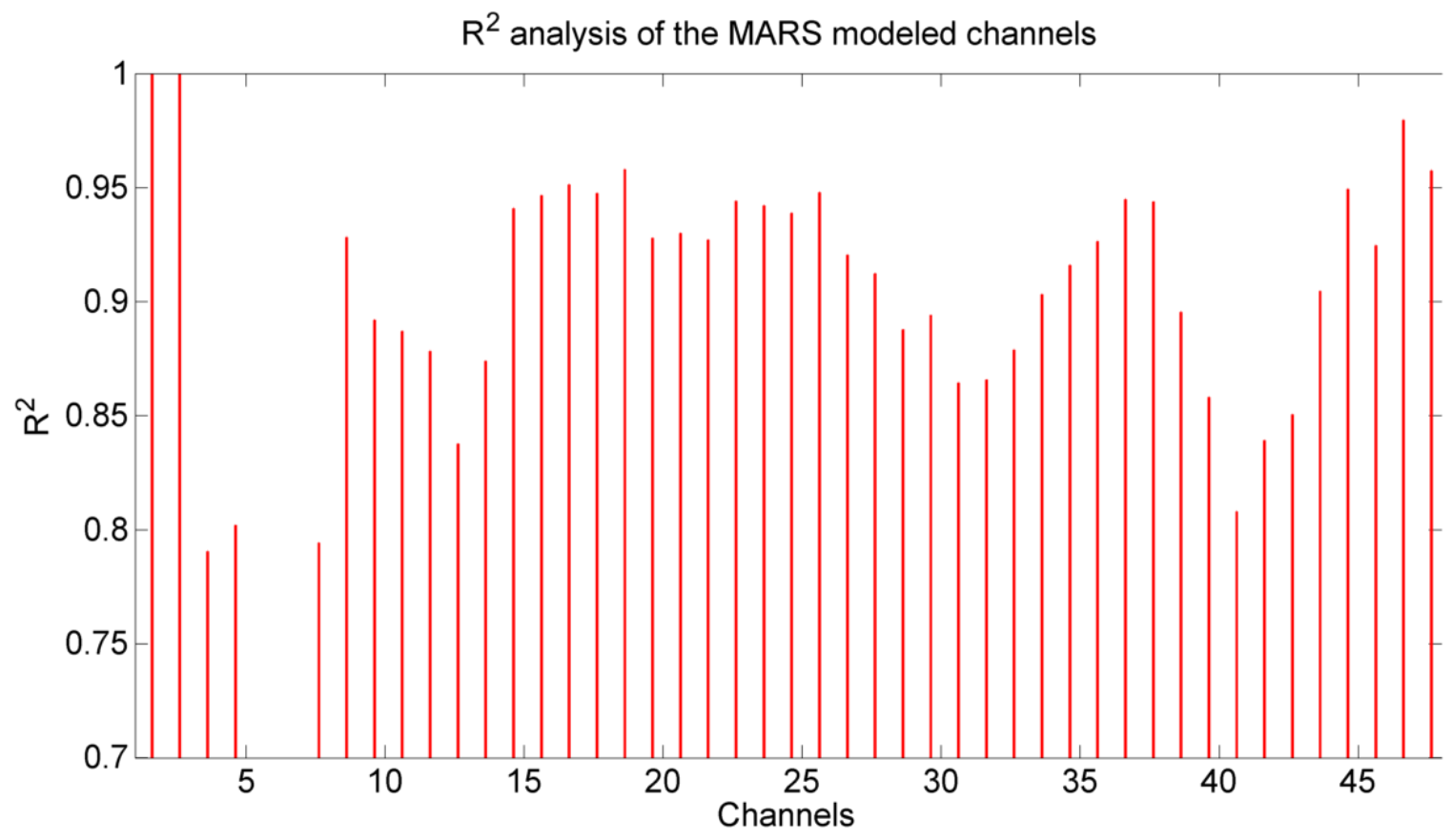

Figure 6-13: Residual sum of squares analysis for the 48 channels for the Post-2007 vehicle during the $20 \mathrm{mph}$ test.

The combination of the vehicle's cracked DPF and the load engine required for this test reduces the chances for the nucleation mode to occur. Both pathways close in early and late stages of the plume exhibiting a single mode PM distribution (see Figure 6-14). 


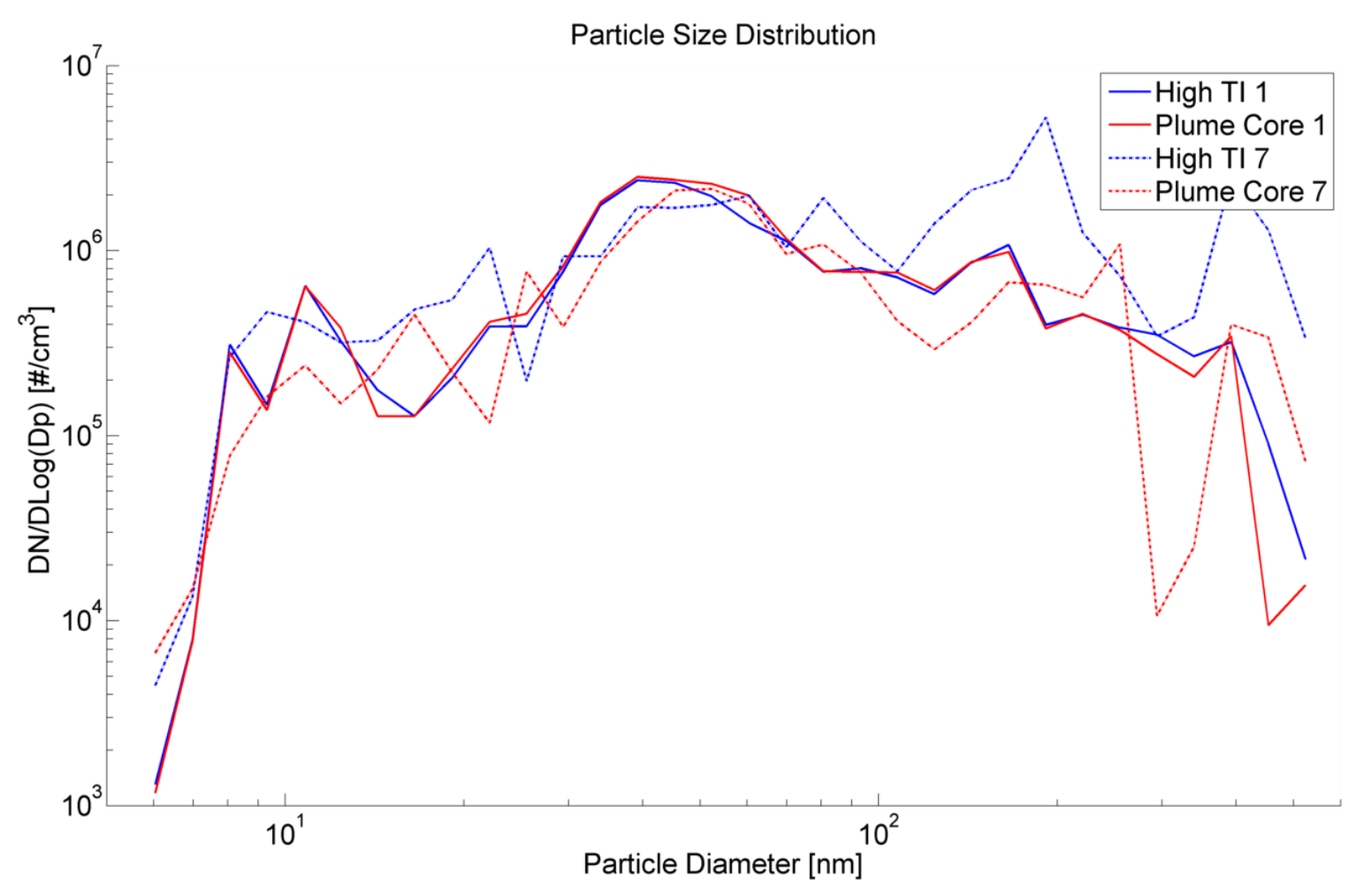

Figure 6-14: Particle size distribution and concentration for plane 1 and plane 7 of the TI path and core plume path (respectively $0.115 \mathrm{~m}$ and $2.815 \mathrm{~m}$ from the exhaust stack). This plot represents the results of the MARS model for the Post-2007 vehicle during the $20 \mathrm{mph}$ test.

These considerations are confirmed by Figure 6-15 where a single mode PM distribution is maintained among all the planes for both paths. 

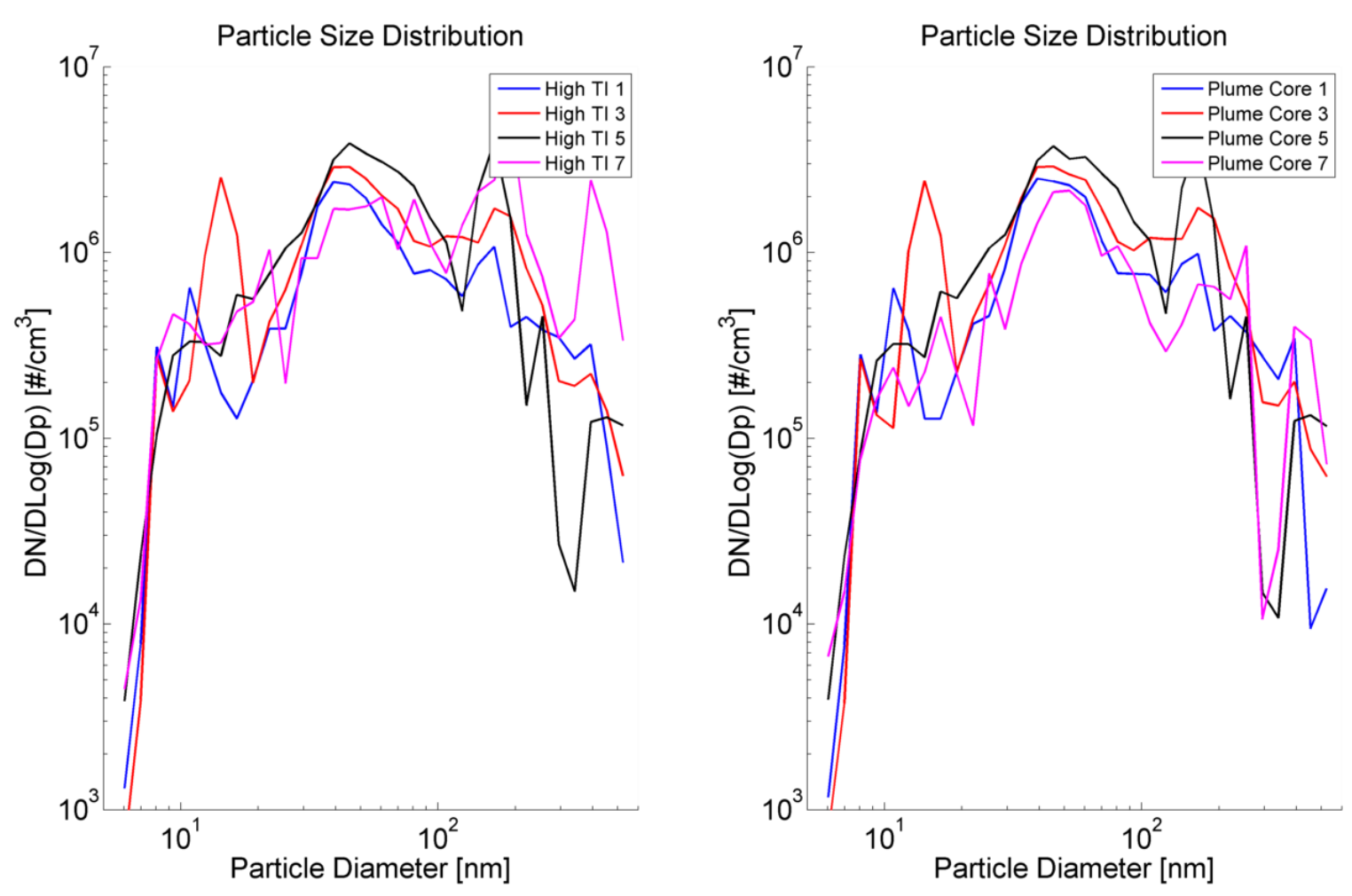

Figure 6-15: Particle size distribution and concentration for plane 1 to plane 7 of the TI path and core plume path (respectively from $0.115 \mathrm{~m}$ to $2.815 \mathrm{~m}$ after the exhaust stack). This plot represents the results of the MARS model for the Post-2007 vehicle during the $20 \mathrm{mph}$ test. 


\subsubsection{35mph Test}

The last test of this vehicle reflects the results of the previous tests, the residual sum of squares for the PM particle size distribution and concentration channels are above 0.85 (see Figure 6-16).

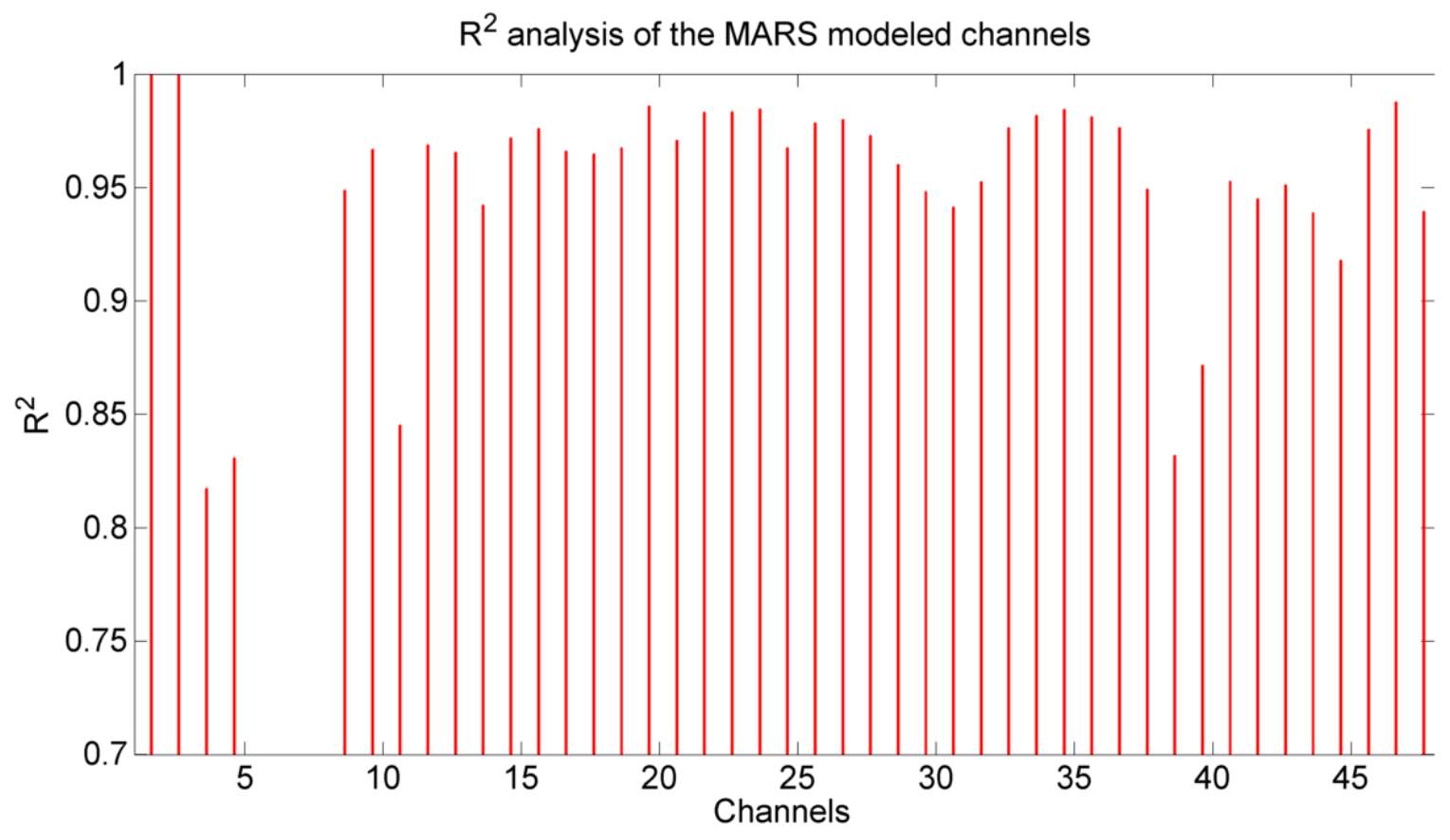

Figure 6-16: Residual sum of squares analysis for the 48 channels for the Post-2007 vehicle during the $35 \mathrm{mph}$ test.

In Figure 6-17, plane 1 traces of PM particle size distribution and concentration present an evident nucleation mode for both paths, but the whole traces result one order of magnitude greater than the ones of plane 7. It is possible to extrapolate that the presence of high turbulent energy in the flow provokes the nucleation mode generation across the early stages of the plume. These indications should induce overlapping for all four traces 
(already noticed in previous cases), but a shift in the model does not make this visible. The cause of this shift can be referred to the relatively small dimensions and high growth rate of the plume within the early stages (see Figure 5-3), which the models partially failed to represent. Furthermore, this shift is confirmed by Figure 6-18 showing that such a shift is present only for the first plane while all the other traces overlap. In addition, it also confirms that the nucleation mode generated at the exhaust stack exit is not affected along the plume.

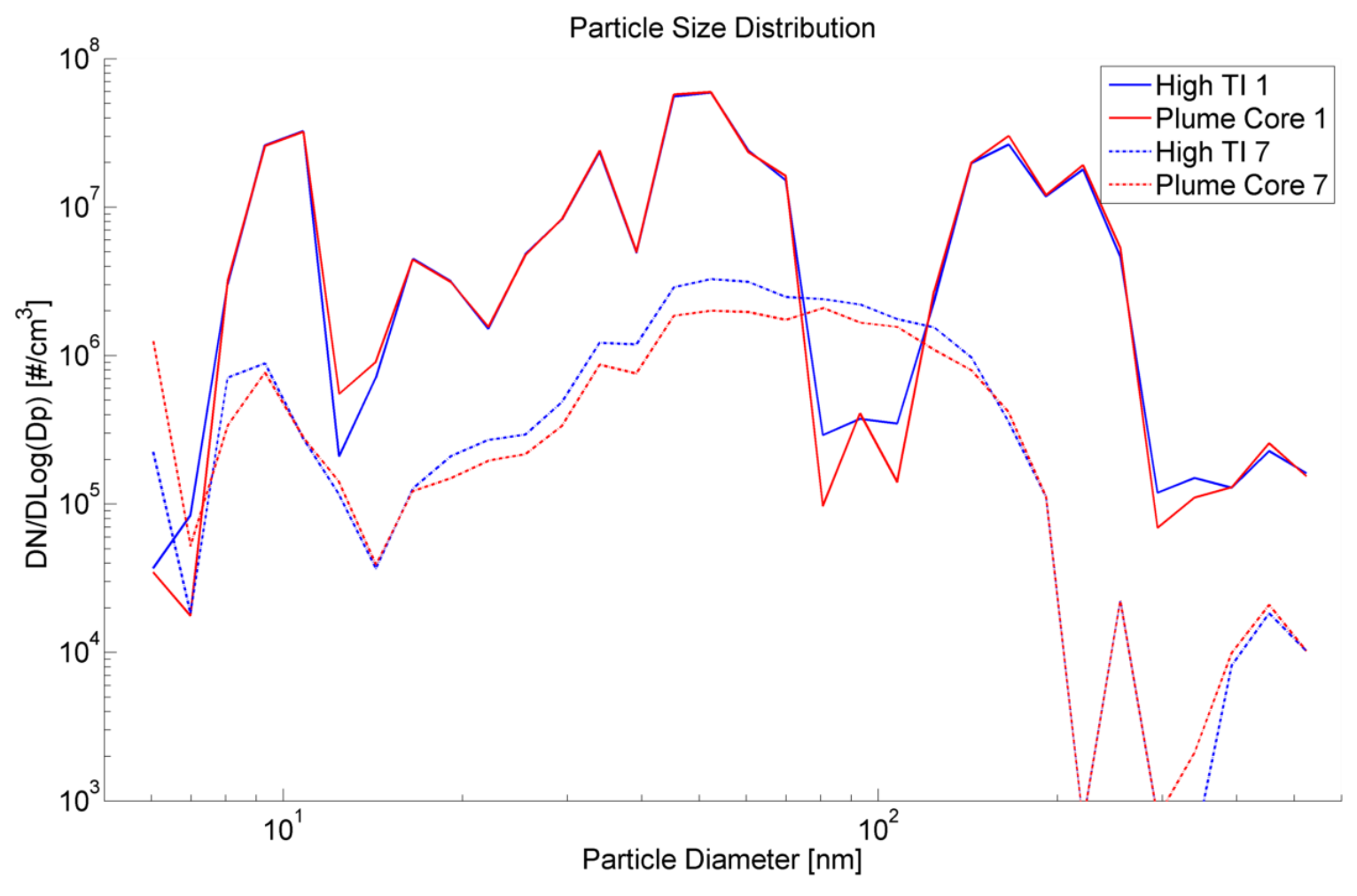

Figure 6-17: Particle size distribution and concentration for plane 1 and plane 7 of the TI path and core plume path (respectively $0.115 \mathrm{~m}$ and $2.815 \mathrm{~m}$ from the exhaust stack). This plot represents the results of the MARS model for the Post-2007 vehicle during the $35 \mathrm{mph}$ test. 

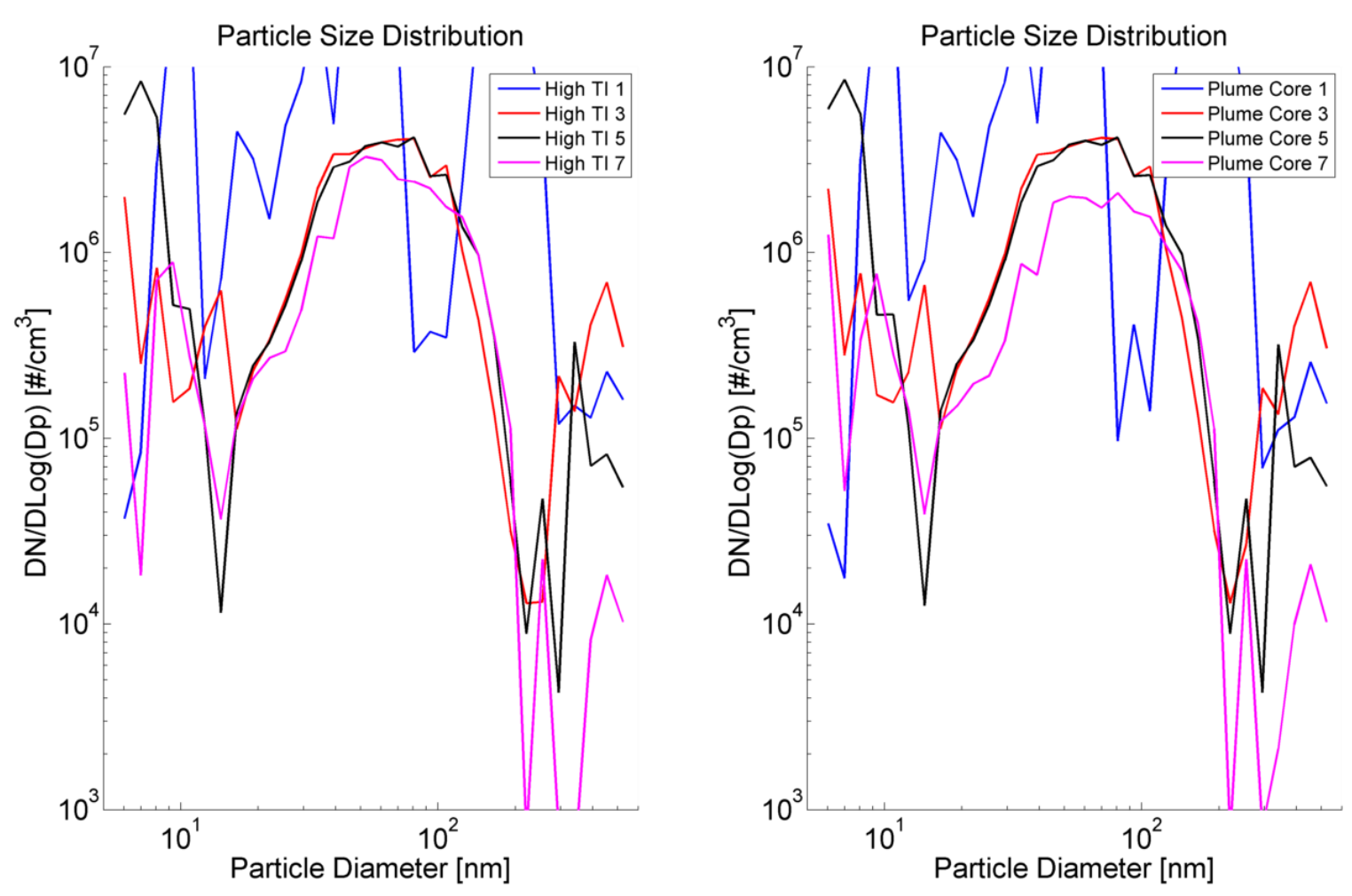

Figure 6-18: Particle size distribution and concentration for plane 1 to plane 7 of the TI path and core plume path (respectively from $0.115 \mathrm{~m}$ to $2.815 \mathrm{~m}$ after the exhaust stack). This plot represents the results of the MARS model for the Post-2007 vehicle during the $35 \mathrm{mph}$ test. 


\subsubsection{Post-2010 Vehicle MARS Results}

\subsubsection{Idle Test}

The PM emissions observed for this combination of vehicle and wind speed are lower than the background air, resulting in models that are not capable of depicting the plume behavior. Figure 6-19 clearly indicates how the majority of the PM models do not reach residual sums of squares greater than 0.9. These models are not capable to fully represent the plume. Such results are not determined by the lack of accuracy of the MARS methods, but from the physical phenomena that was also pushing the PM instrumentation to its lower threshold.

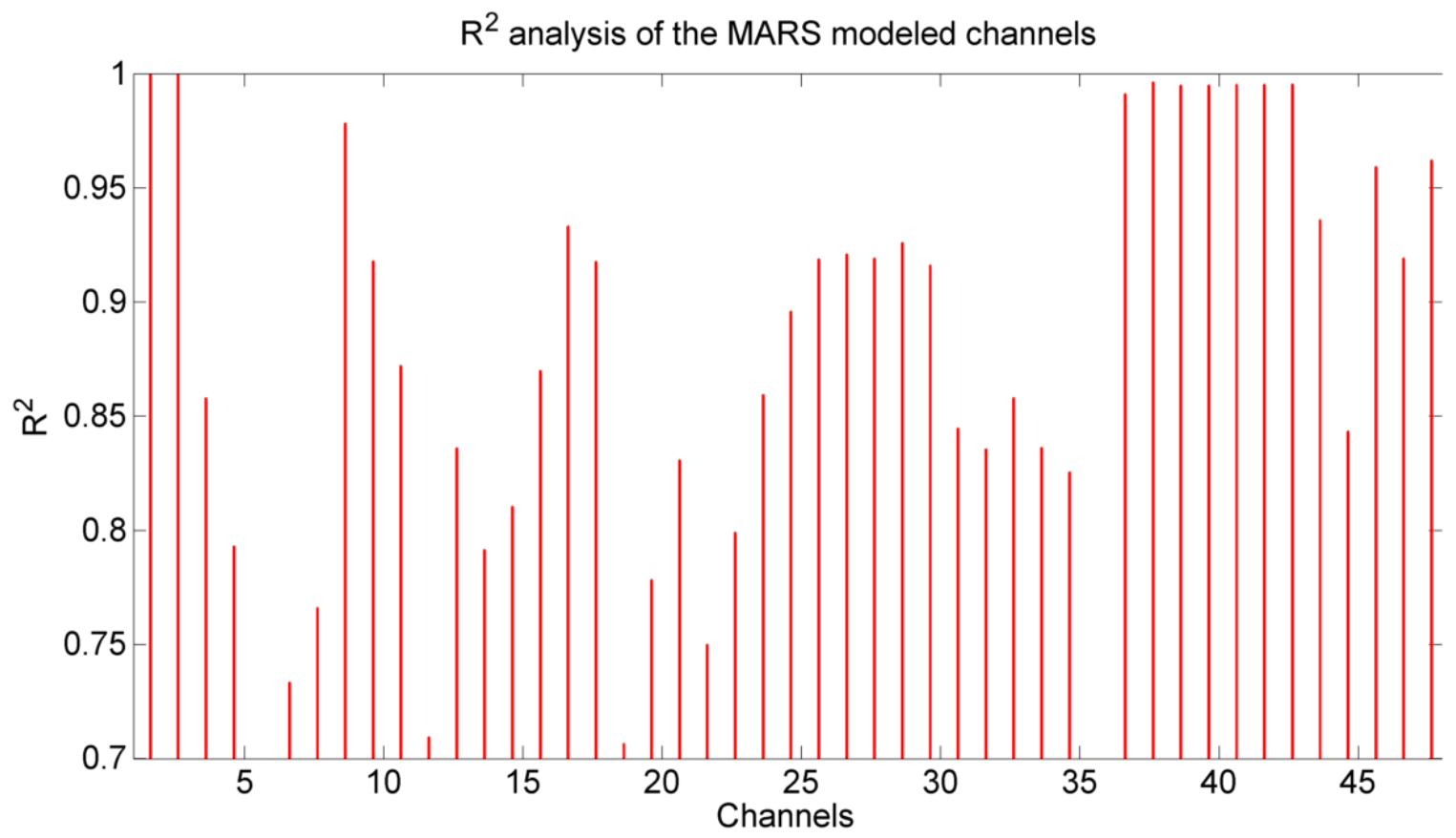

Figure 6-19: Residual sum of squares analysis for the 48 channels for the Post-2010 vehicle during the idle test. 


\subsubsection{2. $20 \mathrm{mph}$ Test}

The analysis of the residual sum of squares for this test specifies that the models are capable to depict the plume with a value greater than 0.85 (see Figure 6-20).

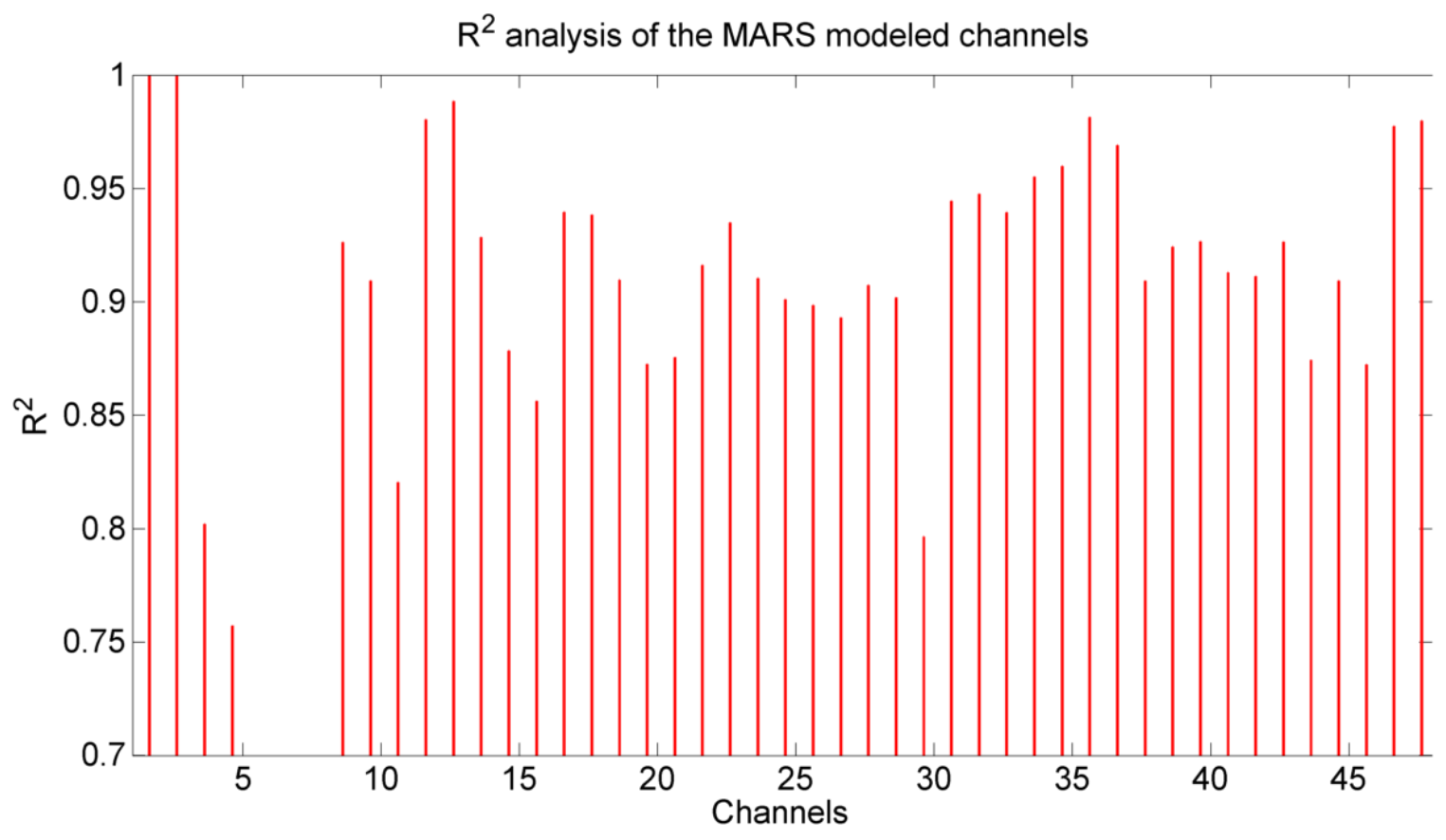

Figure 6-20: Residual sum of squares analysis for the 48 channels for the Post-2010 vehicle during the $20 \mathrm{mph}$ test.

The very low PM emissions from this engine do not allow for stating considerations regarding the nucleation and accumulation mode configuration, as it is possible to observe in Figure 6-21. 


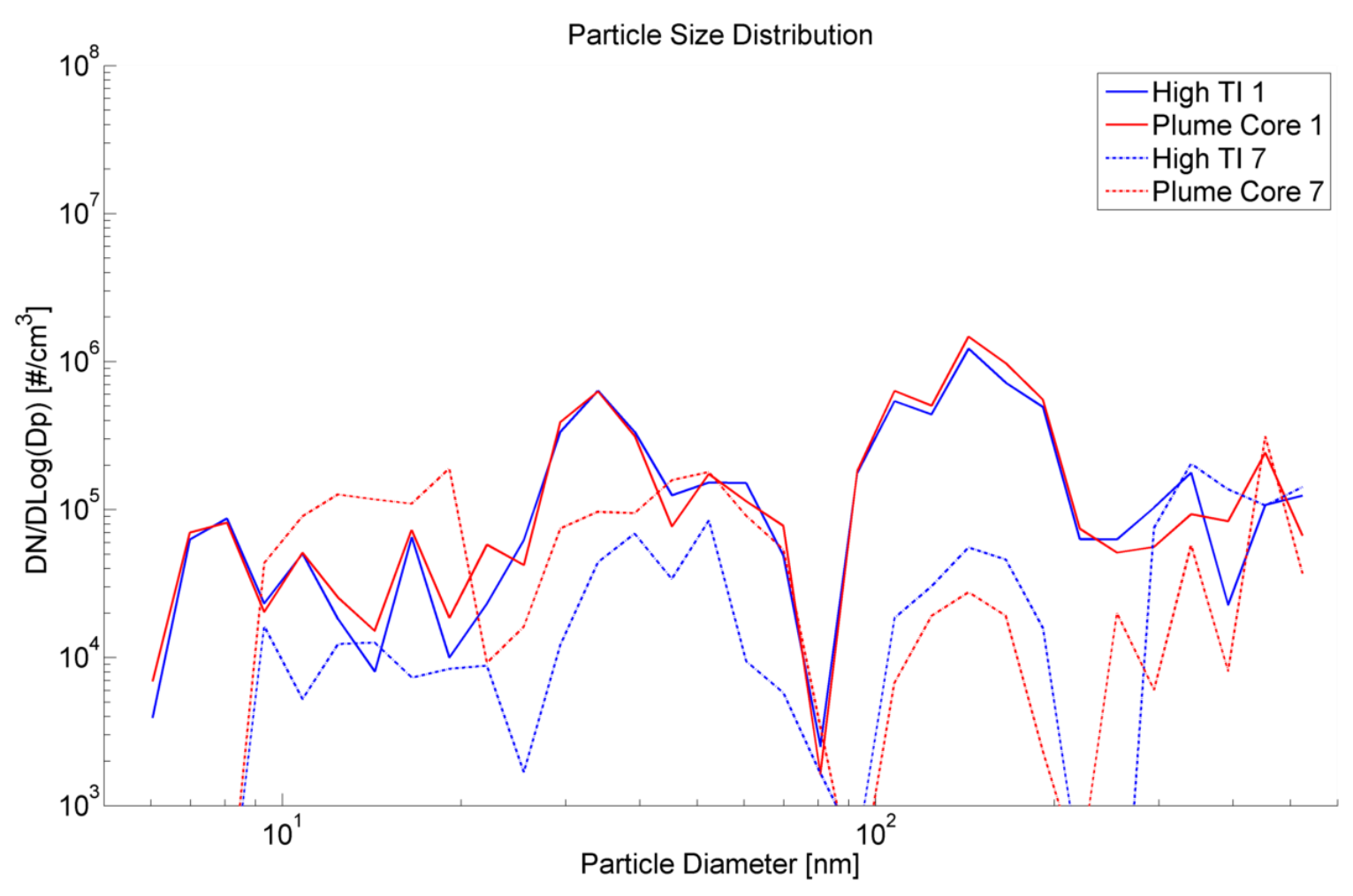

Figure 6-21: Particle size distribution and concentration for plane 1 and plane 7 of the TI path and core plume path (respectively $0.115 \mathrm{~m}$ and $2.815 \mathrm{~m}$ from the exhaust stack). This plot represent the results of the MARS model for the Post-2010 vehicle during the $20 \mathrm{mph}$ test. 


\subsubsection{35mph Test}

The results of the last test described in this chapter show the best performance in terms of residual sum of squares of the MARS models. Figure 6-22 indicates that the PM particle size distribution and concentration channels have $\mathrm{R}^{2}>0.9$.

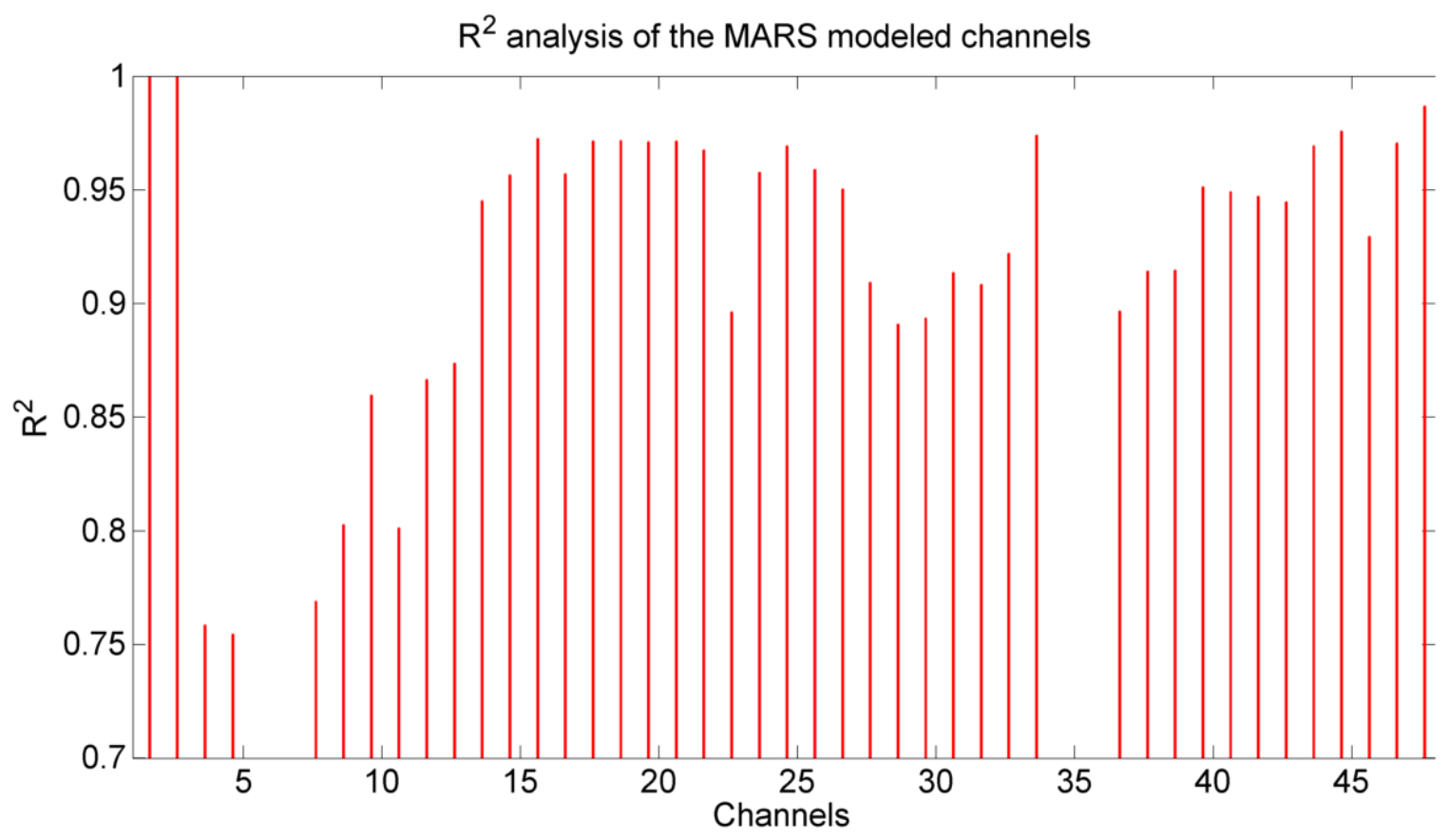

Figure 6-22 Residual sum of squares analysis for the 48 channels for the Post-2010 vehicle during the idle test.

The traces of plane 1 show a distinct nucleation mode with peak concentration almost two orders of magnitude greater than the accumulation mode (see Figure 6-23). This result is a good reflection of the concepts indicated over this entire work, the increased load with low soot in the plume set the basis for greater nucleation mode. It is important to 
remark that plane 1 traces show values that are shifted upward, still due to the complexity of the plume in the very early stage.

In addition, in Figure 6-24 the traces for plane 3 present an evident shift and the absolute value of PM concentration is more reliable for plane 5 and 7 . So shifting by two orders of magnitude the traces of plane 1 and of both path in Figure 6-24, it is possible to notice that the nucleation mode PM particles tends to coagulate or be absorbed along the plume.

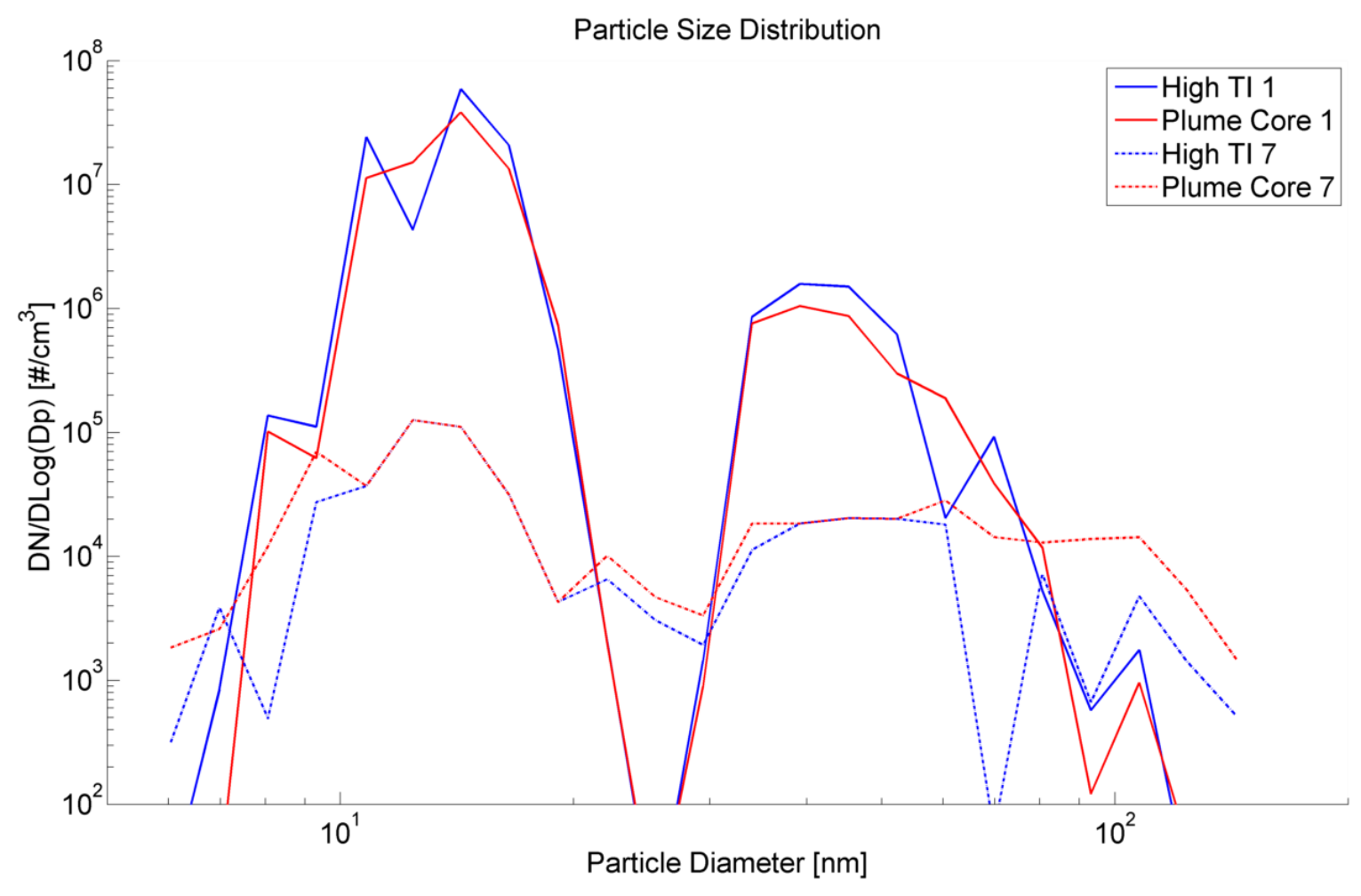

Figure 6-23 Particle size distribution and concentration for plane 1 and plane 7 of the TI path and core plume path (respectively $0.115 \mathrm{~m}$ and $2.815 \mathrm{~m}$ from the exhaust stack). This plot represents the results of the MARS model for the Post-2010 vehicle during the $35 \mathrm{mph}$ test. 

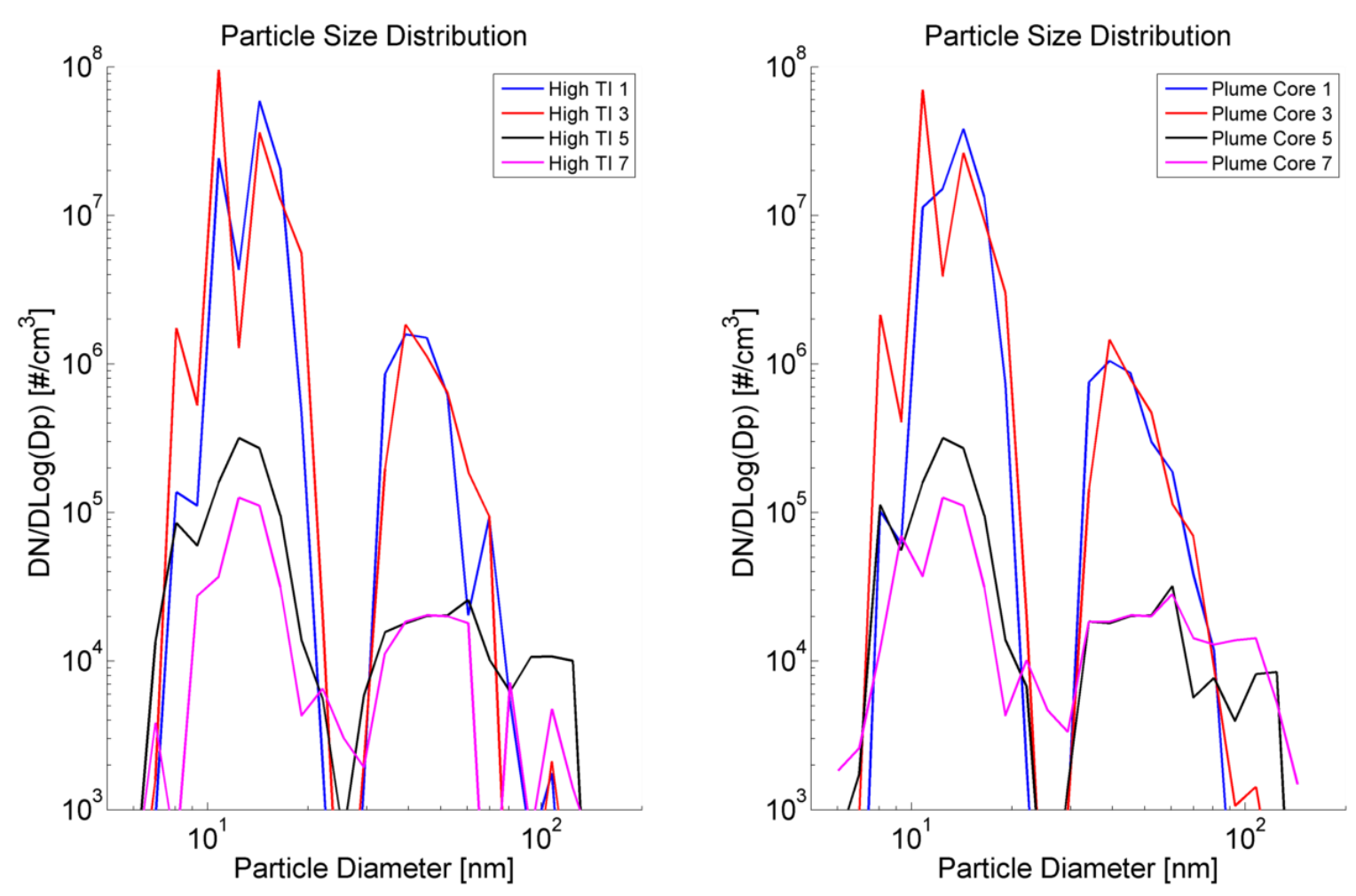

Figure 6-24: Particle size distribution and concentration for plane 1 to plane 7 of the TI path and core plume path (respectively from $0.115 \mathrm{~m}$ to $2.815 \mathrm{~m}$ after the exhaust stack). This plot represents the results of the MARS model for the Post-2010 vehicle during the 35mph test. 


\section{Chapter 7: Conclusions}

WVU's environmental subsonic wind tunnel, designed and built in-house, acted as the essential element in measuring and recording plume characteristics of exhaust. The tunnel was instrumental in determining how real-world conditions affect PM formation and how TI affected nucleation mode particles. The wind tunnel was targeted with wind speeds in excess of $35 \mathrm{mph}$ and with a TI of less than 1.5 percent. The BL indications and recommendations, performed by Rae et al. (1984) and White (1991) were confirmed by experimental data and no interactions between the plume and BL were identified during the commissioning testing or the actual test campaign. As a result, the plume was able to develop in the wake of a class-8 HDDT without walls effect, simulating as close as possible real-world conditions. The sampling probe was able to achieve a positioning accuracy of less than $5 \mathrm{~mm}$, while provoking negligible effects on the plume during sampling.

Raw exhaust, background air, and the developing plume were the three main flows researched during the study. Throughout the test campaign, which consisted of three months, little to no variation occurred in gaseous background air components. This assumption became evident due to $\mathrm{CO}_{2}$ being stable at $450 \mathrm{ppm}$ while $\mathrm{NO}_{\mathrm{x}}$ was stable at 1ppm (within the noise level of the instrument). The background air PM was found to be in a constant behavior with particle concentrations ranging between $1 \times 10^{1}$ and $1 \times 10^{3} \# / \mathrm{cm} 3$ for particle diameters ranging from $5 \mathrm{~nm}$ to $500 \mathrm{~nm}$. The WVU subsonic wind tunnel was positioned one mile away from major roadways and industrial plants, which provided for steady readings and measurements of background gases and PM components without worrying about air samples becoming contaminated by pollutant spikes. 
The Pre-2007 vehicle emitted the highest concentration of PM across all tests, due to the lack of a DPF. Yet, while the pre-2007 truck had the highest PM emission count, it also had the lowest $\mathrm{CO}_{2}$ emissions. The Pre-2007 vehicle showed increased emissions of $\mathrm{NO}_{\mathrm{x}}$ while $\mathrm{CO}$ and THC emissions reduced with increased load, due to improved and hotter combustion taking place relative to the vehicle's specific weight.

The Post-2007 vehicle showed the highest $\mathrm{CO}_{2}$ emissions for every test due to the larger displacement the truck possessed and its relative rated power output. Engine inputs for the HDDTs in each test were kept as contiguous as possible to one another, yet slight differences and thus varying engine efficiencies led to variance in $\mathrm{CO}_{2}$ emissions. These differences became more apparent at each respective truck's idling condition or state and began to reduce or diminish at higher loads. It is the assumption of the author that more modern HDDTs (Post-2007 and Post-2010) present additional auxiliary services if compared to the Pre-2007 HDDT, accompanied by higher load during idling operations and related higher $\mathrm{CO}_{2}$ emissions.

The Post-2007 vehicle was believed to suffer from a partially damaged DPF, yet it possessed improved gaseous emissions in comparison to the Pre-2007 truck; while conversely- the PM emissions of the Post-2007 vehicle were higher than that of the Post2010 truck which had a DPF with no faulty indications. PM soot emissions from the Post2010 vehicle fell below the detectable limit and they were thus unreadable.

Both the Post-2007 and Post-2010 trucks during the idle test showed higher gaseous emissions than did the Pre-2007 vehicle, due to the lack of thermo/chemical activation of the DOC and SCR, save for THC. Gaseous emissions from the Post-2010 vehicle were 
substantially lower than the Post-2007 vehicle explicitly at the $35 \mathrm{mph}$ test phase. The Post2010 vehicle's aftertreatment system proved to be one marked by high efficiency during the $35 \mathrm{mph}$ test, wherein the gaseous analyzers only recorded $\mathrm{CO}_{2}$.

The third flow, and focus of this study, requires conclusion statements per each combination of truck and wind speed due the variation in exhaust composition and interaction with background air. The measurements obtained from plume sampled in the wind tunnel largely agreed with the literature of those who pioneered similar research. Experimental modeling work, proposed by Gautam et al. (2003) and Kim et al. (2001), indicated that the most important events in PM nanoparticle formation and evolution occur within $2 \mathrm{~m}$ from the exhaust stack; while Rönkkö et al. (2006) suggested that on-road chase studies elicit no differences in PM as it is observed after $5 \mathrm{~m}$ from the exhaust. It is imperative to note that these processes, the relative distance from the exhaust and the corresponding PM findings, are dependent upon the balance between the EC and $\mathrm{OC}$ in the raw exhaust and the TI surrounding the exhaust.

In comparing the differences between plume PM measurements and standardized laboratory techniques, striking differences in nucleation mode formation occurred; as seen in Maricq et al. (2001). Plume boundary characteristics and the local DR were crosschecked across several different analyzers. $\mathrm{CO}_{2}$ and $\mathrm{NO}_{\mathrm{x}}$ provided the most accurate information as it pertains to plume shape, dimensions, and concentration gradient. $\mathrm{NO}_{\mathrm{x}^{-}}$ based DR was adopted for the Pre-2007 and Post-2007 vehicles, while a $\mathrm{CO}_{2}$ based DR was used with the Post-2010 HDDT (lack of $\mathrm{NO}_{\mathrm{x}}$ emissions). 
The Pre-2007 HDDT is not equipped with a DPF, and Kittelson (1999) indicated that generally in such a situation the solid compounds of the PM tend to absorb the volatile compounds, inhibiting them from nucleating into nanoparticles. During the idle test, the low engine load and low combustion temperature provoked low levels of soot and relatively high levels of THC. This PM balance towards the volatile compounds set the conditions for the nucleation mode to appear, with a double mode PM particle size distribution and concentration. In the initial stages of plume formation, the area with higher TI (within $0.3 \mathrm{~m}$ from the exhaust) showed a slightly higher nucleation mode particle concentration. The fast decay of the TI further downstream of the exhaust can no longer sustain the generation of new nanoparticles, but provokes only a continuous mixing of heterogonous areas of the plume, until a constant PM composition is achieved across the plume. MARS modeling of the plume confirmed such a statement and it was able to depict higher nucleation mode particle concentrations in the area with a higher TI. Measurements from the DDS test show greater nucleation mode increases than measurements from the plume.

During the 20mph test, significant levels of soot emissions occurred prior to the formation of the nucleation mode in the core of the plume. However, this did not occur in the region with higher TI characterized as being $0.3 \mathrm{~m}$ from the exhaust, which has higher turbulent flow energy than the idle test, resulting in an overall reduction in the potential for nucleation to occur. With that being said, a homogenous PM particle size distribution and concentration is visible across the plume for distances exceeding $.5 \mathrm{~m}$ the outlet of the exhausts stack. 
In the final test phase of this vehicle, the reduction trend experienced by the nucleation mode identified in the $20 \mathrm{mph}$ became more apparent. The tradeoff between increased soot, TI, and reduced volatile compounds led to the appearance of a weak nucleation mode. MARS modeling techniques provided indispensable information illustrating that the newly generated nucleation mode was absorbed within $2 \mathrm{~m}$ from the exhaust stack. The SMPS, adopted with the DDS, did not accurately portray real-world measurements.

The Post-2007 vehicle had a partially damaged DPF which allowed for traces of soot to escape the aftertreatment system. During the idling test phase, small levels of THC and no soot were detected in the raw exhaust, thus high nucleation potential was achieved confirming studies postulated by Kittelson (1999) PM emissions were substantially lower for the Post-2007 vehicle and a predominant nucleation mode was present, one characterized as one order of magnitude greater than the accumulation mode. Due to this configuration, the PM emissions proved to be slightly lower than the background PM.

The high TI region's nucleation mode was greater at the core of the plume. The difference quickly faded within $0.25 \mathrm{~m}$ from the exhaust stack, leading to a constant PM particle size distribution and concentration for the entire plume. Both SMPS and DMS failed to depict the nucleation mode identified in the plume. It is the idea of the author that background air, richer in solid nuclei than the HEPA-filtered air of the DDS, promoted greater heterogenic nucleation. Large PM emissions were measured from the $20 \mathrm{mph}$ test phase, but these emission measurements possessed a similar ratio to both the nucleation mode and accumulation mode of the idle test phase. It can be construed that the high 
nucleation potential of this raw exhaust, combined with higher levels of TI, indicates a nucleation mode maximized in low TI regions of the plume within $0.25 \mathrm{~m}$ of the exhaust.

MARS modeling was instrumental in predicting that a substantial amount of nucleation mode formation and evolution began in each part of the plume within $.15 \mathrm{~m}$ of the exhaust. Yet, MARS made apparent and thus underlined strong adsorption of the nucleation mode as it occurred $2 \mathrm{~m}$ from the exhaust stack. Furthermore, the regeneration event observed halfway through this test showed an increase of two orders of magnitude of the nucleation and accumulation modes, while the region with the highest TI had the greatest nucleation mode. The high exhaust temperature registered at the DOC (in excess of $475^{\circ} \mathrm{C}$ ) suggests the nucleation mode during the regeneration event might be composed of $\mathrm{H}_{2} \mathrm{SO}_{4}$ due to the much higher temperature in the exhaust, as indicated by Rönkkö et al. (2006), even for low fuel-sulfur concentrations. DDS measurements positively reported on the presence PM emissions post-adsorption, but not the emissions during the regeneration or initial formation of the nucleation mode. Higher soot emissions were observed in the $35 \mathrm{mph}$ test, due in part to the damaged DPF and the regeneration event that preceded the test phase. These increased soot emissions are reflected in lower nanoparticles, wherein the high TI region presented slightly higher values if compared to the core of the plume. The laboratory standard DDS measurements show overlapping data with the accumulation mode, but they fail to represent the nucleation mode.

The Post-2010 vehicle, utilizing a state-of-the-art aftertreatment system, presented extremely low PM emissions difficult to detect with standard automotive PM measurements devices. The lowest PM emissions measurements of the entire test campaign 
were found in the $20 \mathrm{mph}$ test. The plume shown PM emission lower than the background air by one order of magnitude, which did not provide for considerations on MARS performance or a comparison with results of standardized laboratory techniques. During the $35 \mathrm{mph}$ test, the increased load sustained by the truck barely sufficed to produce substantial PM emissions that would allow for accurate measurements. Analysis of the MARS modeling system indicates that the complete lack of soot in the raw exhaust, combined with higher TI, produced a marked nucleation mode within $0.1 \mathrm{~m}$ from the exhaust stack; which was absorbed by the background PM within $1.5 \mathrm{~m}$ from the exhaust outlet. The comparison with DDS measurements illustrates the lack of laboratory techniques that could have been used to depict the occurrence of nucleation mode when compared to real-world operating conditions for each respective truck. 


\section{Chapter 8: Future Recommendations}

This work provided an extensive amount of information regarding the formation and evolution of PM in real-world conditions. Analyses provided several indications of a link between TI in the plume and formation of the nucleation mode. On the other hand, several other aspects did not have a chance to be better investigated during this work. In the following bullet points, the author suggests a series of recommendations that could be pursued in the future:

- The interaction of the exhaust gases with the background air in some cases is more rapid than expected, future works should investigate the area within 15 $\mathrm{cm}$ from the exhaust stack (i.e. increase the amount of sampling points).

- A statistical correlation between TI and nucleation mode formation should be performed, via linear regression or MARS.

- The PM particle size distribution and concentration results should be compared with CVS measurements, bettering evaluation differences between real-world measurements and laboratory standards techniques.

- Higher loads and wind speeds should be investigated (i.e. 55 and $70 \mathrm{mph}$ )

- Effect of relative humidity (RH) on PM emissions thru spay of water vapor at the inlet of the WVU wind tunnel.

- Investigation of exhaust elements to provide a gradual cooling of the exhaust gases prior the injection into the background air. 


\section{Glossary}

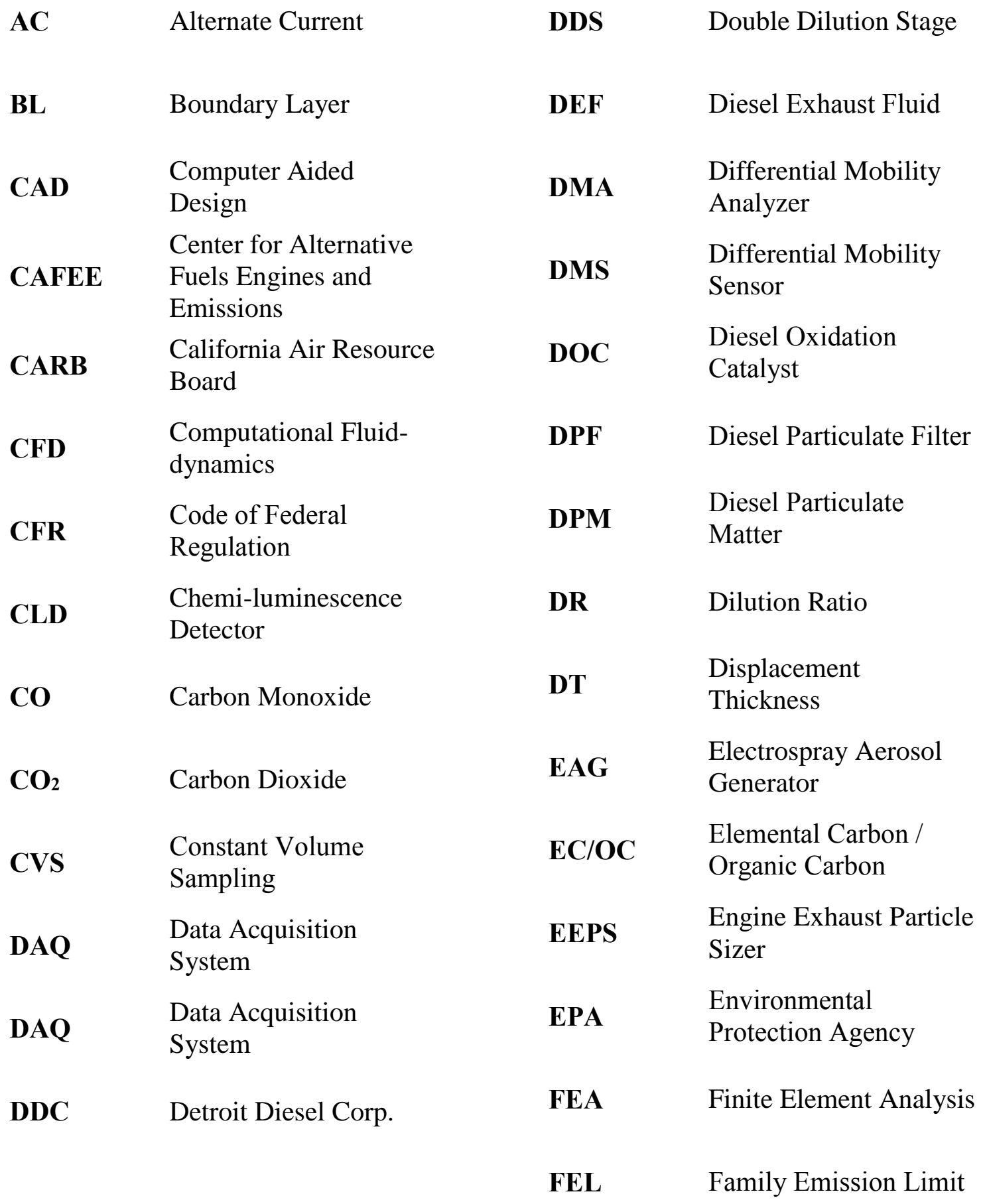




\begin{tabular}{|c|c|c|c|}
\hline FEM & Finite Element Method & JRC & $\begin{array}{l}\text { European Joint } \\
\text { Resource Council }\end{array}$ \\
\hline FID & $\begin{array}{l}\text { Flame Ionization } \\
\text { Detector }\end{array}$ & LES & $\begin{array}{l}\text { Large Eddies } \\
\text { Simulation }\end{array}$ \\
\hline FIM & Failure Index Method & LHDDE & $\begin{array}{l}\text { Light Heavy-Duty } \\
\text { Diesel Engine }\end{array}$ \\
\hline FTP & $\begin{array}{l}\text { Federal Testing } \\
\text { Protocol }\end{array}$ & MARS & $\begin{array}{l}\text { Multivariate Adaptive } \\
\text { Regression Splines }\end{array}$ \\
\hline GVW & Gross Vehicle Weight & MHDDE & $\begin{array}{l}\text { Medium Heavy-Duty } \\
\text { Diesel Engine }\end{array}$ \\
\hline GVWR & $\begin{array}{l}\text { Gross Vehicle Weight } \\
\text { Rating }\end{array}$ & MY & Model Year \\
\hline $\mathrm{HC}$ & Hydrocarbon & NDIR & $\begin{array}{l}\text { Non-dispersive Infra- } \\
\text { Red }\end{array}$ \\
\hline HDDE & $\begin{array}{l}\text { Heavy-duty Diesel } \\
\text { Engine }\end{array}$ & NIOSH & $\begin{array}{l}\text { National Institute for } \\
\text { Occupational Safety } \\
\text { and Health }\end{array}$ \\
\hline HDDT & $\begin{array}{l}\text { Heavy-duty Diesel } \\
\text { Truck }\end{array}$ & NIST & $\begin{array}{l}\text { National Institute of } \\
\text { standard and testing }\end{array}$ \\
\hline HDDV & $\begin{array}{l}\text { Heavy-duty Diesel } \\
\text { Vehicle }\end{array}$ & NOx $_{x}$ & Oxides of Nitrogen \\
\hline HDTT & $\begin{array}{l}\text { Heavy Duty Transient } \\
\text { Test }\end{array}$ & NTE & Not to Exceed \\
\hline HEPA & $\begin{array}{l}\text { High Efficiency Particle } \\
\text { Air }\end{array}$ & $\mathbf{P M}$ & Particulate Matter \\
\hline HFID & $\begin{array}{l}\text { Hydrocarbons Flame } \\
\text { Ionization Detector }\end{array}$ & PMP & $\begin{array}{l}\text { Particle Measurements } \\
\text { Programme }\end{array}$ \\
\hline HHDDE & $\begin{array}{l}\text { Heavy Heavy-Duty } \\
\text { Diesel Engine }\end{array}$ & SCR & $\begin{array}{l}\text { Selective Catalyst } \\
\text { Reduction }\end{array}$ \\
\hline IARC & $\begin{array}{l}\text { International Agency } \\
\text { for Research on Cancer }\end{array}$ & SET & $\begin{array}{l}\text { Supplemental } \\
\text { Emissions Test }\end{array}$ \\
\hline
\end{tabular}




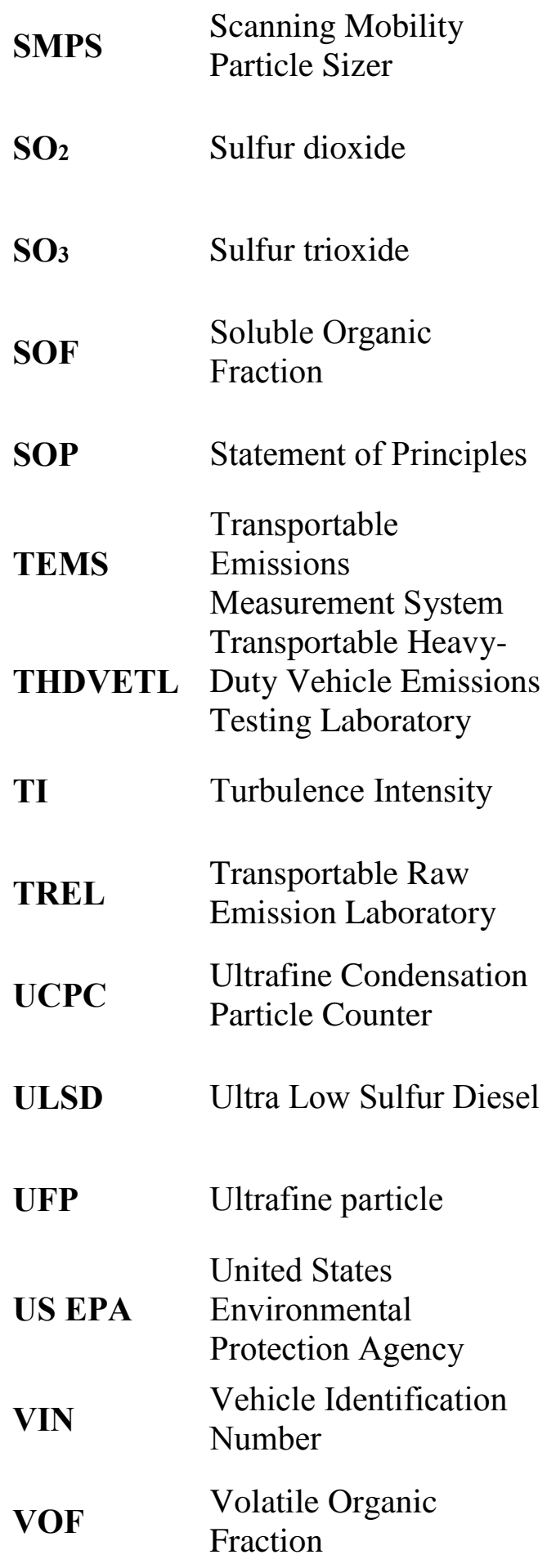




\section{Bibliography}

Abu-Allaban, M., \& Coulomb, W. (2002). Exhaust Particle Size Distribution Measurements at the Tuscarora Mountain Tunnel. Aerosol Science and Technology Vol. 36, 771-789.

Alfoldy, B., Giechaskiel, B., Hofmann, W., \& Drossinos, Y. (2009). Size-distribution Dependent Lung Deposition of Diesel Exhaust Particles. Aerosol Science 40, 652663.

Ardanese, R., Ardanese, M., Besch, M. C., Adams, T. R., Thiruvengadam, A., Shade, B. C., . . Miyasato, M. (2009). PM Concentration and Size Distributions from a Heavy-duty Diesel Engine Programmed with Different Engine-out Calibrations to Meet the 2010 Emission Limits. SAE International 2009-01-1183.

Biswas, S., Hu, S., Verma, V., Herner, J. D., Robertson, W. H., Ayala, A., \& Sioutas, C. (2008). Physical Properties of Particulate Matter (PM) from Late Model Heavyduty Diesel Vehicles Operating with Advanced PM And NOx Emission Control Technologies. Atmospheric Environment 42 5622-5634.

Biswas, S., Verma, V., Schauer, J. J., \& Sioutas, S. (2009). Chemical Speciation of PM Emissions from Heavy-duty Diesel Vehicles Equipped with Diesel Particulate Filter (DPF) and Selective Catalytic Reduction (SCR) Retrofits. 43 1917-1925.

Brauer, M., Hoek, G., van Vliet, P., Meliefste, K., Fischer, P. H., Wijga, A., . . . Brunekreef, B. (2002). Air Pollution from Traffic and the Development of Respiratory Infections and Asthmatic and Allergic Symptoms in Children. Journal of Respiratory Critical Care Vol. 166, 1092-1098. 
Britt, A., \& Ayala, A. (2002). Ultrafine PM Emissions from Natural Gas, OxidationCatalyst Diesel, and Particle-Trap Diesel Heavy-Duty Transit Buses. Environ. Sci. Technol. 36 5041-5050.

Brunekreef, B., Janssen, N. A., de Hartog, J., Harssema, H., Knape, M., \& and van Vliet, P. (1997). Air Pollution from Truck Traffic and Lung Function in Children Living near Motorways. Epidemiology, Vol. 8, 298-303.

Burtscher, H. (2001). Tailpipe Particulate Emissions Measurement for Diesel Engines. Windisch: Fachhochschule Aargau, University of Applied Science.

Casati, R., Volker, S., Vogt, R., \& Benter, T. (2007). Measurement of Nucleation and Soot Mode Particle Emission. Atmospheric Environment 41 (2007) 2125-2135.

El-Fadel, M., \& and Hashisho, Z. (2001). Vehicular Emissions in Roadway Tunnels: A Critical Overview. Critical Reviews in Environmental Science and Technology Vol. 31, 125-174.

Englert, N. (2004). Fine Particles and Human Health: a Review of Epidemiological Studies. Toxicology Letters, Vol. 149, 235-242.

Ferrero, L., Mocnick, B., Perrone, M., Sangiorgi, G., \& Bolzacchini, E. (2011). Vertical profiles of Aerosol Absorption Coefficient from micro-Aethalometer Data and Mie Calculation over Milan. Science of the Total Environment 409, 2824-2837.

Finkelstein, M. (2003). Concentrations and Size Distributions of Particulate Matter Emissions from a Class-8 Heavy-duty Diesel Truck Tested in a Wind Tunnel. SAE Transactions, Journal of Fuels and Lubricants, 1615-1634.

Friedman, J. H. (1990). Multivariate Adaptive Regression Splines Tech Report 102. Stanford, CA: Department of Statistics Stanford University.

Friedman, J. H. (1993). Fast MARS Tehnical Report No. 110. Stanford, CA: Department of Statistics Standford University.

Gautam, M., Clark, N., Mehta, S., Boyce, J., Rogers, F., \& Gertler, A. (2003). Concentrations and Size Distributions of Particulate Matter Emissions from a 
Class-8 Heavy-duty Diesel Truck Tested in a Wind Tunnel. SAE Transactions, Journal of Fuels and Lubricants, 1615-1634.

Giechaskiel, B., Ntziachristos, L., Samaras, Z., Volker, S., Casati, R., \& V., R. (2005). Formation Potential of Vehicle Exhaust Nucleation Mode Particles On-road and in the Laboratory. Elsevier Atmospheric Environment 39 3191-3138.

Gilmour, M., McGee, J., Duvall, R., Dailey, L., Daniels, M., Boyskin, E., . . Devlin, R. (2007). Comparative Toxicity of Size-Fractionated Airborne. Inhalation Toxicology 19 (Suppl. 1), 7-16.

Hinds, C. W. (1999). Aerosol Technology: Properties, Behavior, and Measurements of Airborne Particles (Vols. ISBN 0-471-19410-7). Los Angeles: WileyInsterscience Publication.

IBC Counsil. (2009). Internation Building Code. IBC Council, Inc.

Jacobson, M. Z., \& Seinfeld, J. H. (2004). Evolution of Nanoparticle Size and Mixing state near the Point of Emission. Atmospheric Environment 38 (2004) 1839-1850.

Kim, D., Gautam, M., \& and Gera, D. (2001). On the Prediction of Concentration Variations in a Dispersing Heavy-duty Truck Exhaust Plume Using k-e Turbulent Closure. Atmospheric Environment Vol. 35, 5267 - 5275.

Kittelson, D., \& Abdul-Khalek, I. (1999). Formation of Nanoparticles during Exhaust Dilution. EFI Members Conference:"Fuels, Lubricants Engines, \&Emissions". University of MInnesota.

Kittelson, D., Ayala, A., \& Olson. (2002). Diesel Aerosol Sampling Methodology - CRC E-43.

Kittelson, D., Hands, T., Nicoklaus, C., Collings, N., Twigg, M., \& Niemela, V. (n.d.). Mass Concentration of Engine Emissions with Spectral Instruments.

Kittelson, D., Johnson, J., Watts, W., Wei, Q., Drayton, M., Paulsen, D., \& Bukowiecki, N. (2000). Diesel Aersol Sampling in the Atmosphere. 
Kittlelson, D. B. (1998). Engines and Nanoparticles: a Review. J. Aerosol Sci. Vol. 29(No. 5/6, pp. 575Đ588).

Kulmala, M., Petäjä, T., Mönkkönen, P. I., Koponen, M., Dal Maso, P. P., Aalto, K. E., \& Lehtinen, J. (2005). On the Growth of Nucleation Mode Particles: Source Rates of Condensable Vapor in Polluted and Clean Environments. Atmos. Chem. Phys., 5, 409-416, 2005.

Littera, D., Besch, M. C., Cozzolini, A., Carder, D. K., Thiruvengadam, A., Sayres, A., . . . Oshinuga, A. (2011). In-use Assessment of a Fresh and Aged SCRT System Retrofitted on a MY1998 Class-8 Tractor. 2011-11ICE-0232. SAE International.

Littera, D., Cozzolini, A., Besch, M., Thiruvengadam, A., \& Gautam, M. (2011). High Temperature Sampling System for Real Time Measurement of Solid and Volatile Fractions of Exhaust Particulate Matter. 2011-24-0191. SAE ICE Technical Conference.

Littera, D., Cozzolini, A., Besch, M., Velardi, M., Carder, D., \& Gautam, M. (2013). Comparison of Particulate Matter Emissions from Different Aftertreatment Technologies in a Wind Tunnel. (2013-24-0175).

Littera, D., Velardi, M., Cozzolini, A., Yoder, G., Besch, M., \& Carder, D. a. (2012). Integrated Physical and Chemical Measurements of PM Emissions of Dispersing Plume Heavy-Duty Diesel Truck: Wind Tunnel Studies (part I: Design and Commissioning). ICEF2012-92091. ASME Technical Paper.

Liu, B., Pui, D., Rubow, K., \& Szymanski, W. (1985). Electrostatic Effects in Aerosol Sampling and Filtration. Annuals Occupational Hygien Vol. 29, 251-269.

Marchwinska-Wyrwal, E., Dziubanek, G., \& Hajok, I. (2011). The Impact of Air Pollution on Health, Economy, Environment and Agricultural Sources (Vols. ISBN 978-953-307-528-0). InTech.

Maricq, M. M., Chase, R. E., \& Xu, N. (2001). A Comparison of Tailpiepe, DIlution Tunnel, and Wind Tunnel Data in Measuring Motor Vehicle PM. 51:1529-1537. 
Martyr, A. J., \& Plint, M. (1995). Engine Testing. Elsevier.

Ng, I. P., Ma, H., \& David, K. (2007). Comparing Measurements of Carbon in Desel Exhaust Aerosol using the Aethalometer, NIOSH Method 5040, and SMPS. SAE 2007-01-0334. SAE.

Ng, I. P., Ma, H., \& Kittelson, D. (2007). Comparing Measurements of Carbon in Desel Exhaust Aerosol using the Aethalometer, NIOSH Method 5040, and SMPS. SAE 2007-01-0334. SAE.

Nightingale, J. A., Maggs, R., Cullinan, P., Donnelly, L. E., Rogers, D. F., Kinnersley, R., . . Newman-Taylor, A. (2000). Airway Inflammation after Controlled Exposure to Diesel Exhaust Particulates. American Journal of Respiratory Critical Care Medicine Vol. 162, 161-166.

Oxtoby, D. W. (1992). Homogeneous Nucleation: Theory and Experiment. 4(7627). Pope, C. (2007). Mortality Effects of Longer Term Exposure to Fine Particulate Air Pollution: Review of Recent Epidemiological Evidence. Inhalation Toxicology Vol. 19 Suppl. 1, 33-38.

Rae, J., William, H., \& Pope, A. (1984). Low-Speed Wind Tunnel Testing.

Rönkkö, T., Virtanen, A., Vaaraslahti, K., Keskinen, J., Pirjola, L., \& Lappi, M. (2006). Effect of Dilution Conditions and Driving Parameters on Nucleation Mode Particles in Diesel Exhaust: Laboratory and On-road Study. Elsevier, Atmospheric Environment 40 2893-2901.

SAE. (1993). Chemical methods for the measurement of nonregulated diesel emissions. In SAE, SAE Handbook, vol. 3, Engines, Fuels, Lubricants, Emissions and Noise. Warrendale, PA: Society of Automotive Engineers.

Seagrave JC, B. J. (2001). Comparative Acute Toxicities of Particulate Matter and SemiVolatile Organic Compound Fractions of Traffic Tunnel Air. Toxicologist, Vol. $60,192$. 
Seagrave, J. C., McDonald, J. D., Gigliotti, A. P., Nikula, K. J., Seilkop, S. K., Gurevich, M., \& and Mauderly, J. L. (2002). Mutagenicity and in Vivo Toxicity of Combined Particulate and Semivolatile Organic Fractions of Gasoline and Diesel Engine Emissions. Toxicological Science Vol. 70, 212-226.

Silverman, D. T., Samanic, C. M., Lubin, J. H., Blair, A. E., Stewart, P., Vermeulen, R., \& Coble, J. B. (2011). The Diesel Exhaust in Miners Study: A Nested CaseControl Study of Lung Cancer and Diesel Exhaust. 104:1-14.

Talanquer, V. (2002). Nucleation in Gas-Liquid Transitions. 79(7).

Velardi, M. (2013). Investigation of Emission Characteristics during Low Temperature Combustion using Multivariate Adaptive Regression Splines. Morgantown: West Virginia University.

Wang, X., Westerdahl, D., Wub, Y., Pan, X., \& Zhang, K. M. (2011). On-road Emission Factor Distributions of Individual Diesel Vehicles. Atmospheric Environment 45 (2011) $503 e 513$.

White, F. M. (1991). Viscous Fluid Flow. McGraw-Hill Science/Engineering/Math.

WHO. (2012). Diesel Engine Exhaust Carcinogenic. WHO Press Release No. 213.

Wilt, G. (2007). Growth of Diesel Exhaust Particulate Matter in a Ventilated Mine

Tunnel. Morgantown: WVU Department of Mechanical and Aerospace Engineering $\mathrm{PhD}$ Dissertation.

Wu, Y., Carder, D., Shade, B., Atkinson, R., Clark, N., \& Gautam, M. (2009). A CFR 1065-Complinat Transportable/ On-Road Low Emissions Measurement Laboratory with Dual Primary Full-Flow Dilution Tunnels. ICES2009-76090. ASME ICE Spring Technical Conference.

Yu, F., Lanni, T., \& Frank, P. (2004). Measurements of Ion Concentration in Gasoline and Diesel Engine Exhaust. 38 (1417-1423).

Zervas, E., \& Dorlhène, P. (2006). Comparison of Exhaust Particle Number Measured by EEPS, CPC, and ELPI. Aerosol Science and Technology 40, 977-984. 
Zhu, Y., Hinds, W. C., Kim, S., Shen, S., \& Sioutas, C. (2002). Study of Ultrafine Particles Near a Major Highway with with Heavy-duty Diesel Traffic. Atmospheric Environment 36 (2002) 4323-4335. 


\section{Vita}

I obtained my bachelor at the University of Rome "Tor Vergata" in Mechanical Engineering in 2008, and my PhD at West Virginia University in Morgantown in 2014. My field of interest is the automotive world and throughout my academic career special attention was posed on internal combustion engines, gaseous and Pm emissions, PM characterization and morphology correlated to advanced aftertreatment systems and waste heat energy recovery system.

This thesis (report) was typed by Daniele Littera

daniele.littera@gmail.com 